\title{
APPENDIX. CHEMICAL COMPOSITION OF DEEP SEA SEDIMENTS - SITES 9 THROUGH 425, LEGS 2 THROUGH 54, DEEP SEA DRILLING PROJECT
}

\author{
Thomas W. Donnelly, Department of Geological Sciences, State University of New York, Binghamton, New York
}

\section{INTRODUCTION}

In 1971, I began a limited program of analysis of "typical" deep-sea sediments, which was expanded under a grant from the National Science Foundation from 1974 through 1978. In total, about 3000 samples were analyzed; all of the results are reported here. Several analyses have already appeared or will appear in Initial Reports volumes (Volume 15, Donnelly and Nalli, 1973; Volume 33, Donnelly and Wallace, 1976a; Volume 35, Donnelly and Wallace, 1976b; Volumes 51 through 53, Donnelly, in press; and Donnelly, this volume). These earlier results have been modified slightly: the Leg 15 samples have been corrected in a more precise manner for sea-salt contributions, and the Leg 35 results for manganese have been changed slightly to reflect a further evaluation of our manganese standard.

Samples analyzed are representative. In a number of sites, iron- and manganese-enriched specimens have been selected. A series of volcanic ash beds have been included; these are grouped by petrographic characteristics instead of site number. A relatively small number of sandy beds have been analyzed, and these have been identified as such. At a few sites, highly calcareous sediments were analyzed. In our experience, this type of analysis yields information of only marginal significance for samples with calcium carbonate values higher than 95 per cent unless special steps are taken, as was the case with Site 167.

The tables include conventional sample designation (except that the top of the interval is given rather than the entire interval in centimeters), depth to the nearest meter, oxide weight percentages for dried but unwashed samples, and amount of water-soluble chloride. Sodium, potassium, calcium, and magnesium have been corrected for pore-water values of element/chloride ratios where available, and for sea-water values in a few cases where pore-water analyses were not available. In many cases, especially for Phase III Indian Ocean sites, we performed our own pore-water analyses in order to determine the basis for this correction.

The sample names used here are taken for the most part directly from the Initial Reports without modification. The reader will note that the analysis in many cases implies a name different from that reported by the shipboard sedimentologists, especially in the case of those "siliceous oozes" or "diatom oozes" which have a sufficiently high aluminum content to place them in the category of siliceous clays. This discrepancy in nomenclature underlines the problem of selecting a name for a deep-sea sediment in the absence of chemical information.

\section{ANALYTICAL TECHNIQUE}

The analytical technique is that known in the United States as the "rapid method" of Shapiro and Brannock $(1962,1967)$. In our version the sample is fused in a mixture of lithium tetraborate and lithium metaborate (Leonard Shapiro, personal communication), and the bead is dissolved in a weak sulfuric acid solution. Aliquots are analyzed colorimetrically for silicon (Molybdenum Blue), aluminum (calcium Alizarin Red), titanium (Tiron), and phosphorus (Molybdenum Blue with stannous chloride). Our results are apparently similar to those of other laboratories, but we caution anyone using these methods to perform the silicon analysis a relatively short period after the solution of the bead. There is an occasional problem that we interpret as polymerization within the aqueous solution of the sample.

Appropriately diluted aliquots, which have been spiked with lanthanum chloride, are analyzed by absorption flame photometry for calcium, magnesium, sodium, potassium, iron, and manganese. The last two elements can also be analyzed colorimetrically, but we have found no clear advantage in doing so.

All samples are compared to a standard curve made with our own sediment and igneous rock standards. Our sediment standards have been analyzed by the U.S. Geological Survey laboratory with very close agreement. In our procedure, care is taken to dilute samples with the blank solution to bring their elemental responses to the linear portions of the determinative curve.

We do not routinely analyze for $\mathrm{CO}_{2}$ nor $\mathrm{H}_{2} \mathrm{O}^{+}$and the summations are correspondingly low. Iron is reported as total iron expressed as $\mathrm{Fe}_{2} \mathrm{O}_{3}$. The sea-salt correction is made by extracting water-soluble chloride in simmering distilled water and determining the chloride with a mercurous nitrate titration using a diphenyl-carbazone indicator. We have found that apparently not all the chloride is successfully extracted from a previously dried sample, especially in siliceous oozes. Thus, sodium and magnesium especially are undercorrected and are reported as overly high values. Frank Manheim (personal communication) has found an identical result. Our laboratory is now heavily engaged in the study of piston-core samples, which tend to have far higher values of chloride than most DSDP material. We routinely wash our samples once in distilled water, and then analyze the small amount of chloride remaining in order to make the correction as before. In the present group of analyzed samples, the only washed samples were those of Leg 54, which were received by us in an excessively moist state. 
At one site (Site 167) where the calcium carbonate values exceeded 99 per cent, we removed the calcium in EDTA and analyzed the remaining elements. At other sites, we analyzed double aliquots of the original dried sample. For the four sites of Leg 37, which average about 94 per cent calcium carbonate based on our chemical results for 17 samples, we analyzed both single (50-mg) and double (100-mg) aliquots. Table 1 shows that the results differ systematically for this comparative test. The aluminum and iron results are nearly identical, and silicon is very close; the remaining elements show greater discrepancy, with potassium and titanium deviating the most. We regard the analyses of highly calcareous samples in this report as of value principally for aluminum, iron, and silicon, and caution the reader not to place an overly high significance on the other elements. At values of 90 per cent calcium carbonate, the results for all the elements are far more reliable.

To evaluate the accuracy of our technique, on $17 \mathrm{oc}-$ casions the analyst was given a blind sample of a U.S. Geological Survey standard igneous rock. Table 2 shows the average absolute difference between the accepted values for each oxide and our results.

\section{ACKNOWLEDGMENTS}

Funding for this project was provided by the National Science Foundation (OCE-7413196). Samples were provided

TABLE 1

Comparison of Analytical Results for Single $(50-\mathrm{mg})$ and Double $(100-\mathrm{mg})$ Aliquots of 17 Highly Calcareous Samples, Leg 37, DSDP, Expressed as a Ratio

\begin{tabular}{lcc}
\hline Oxide & \multicolumn{5}{c}{ mg Result } & $\begin{array}{c}\text { Average for } \\
\text { 100 mg Result }\end{array}$ & -mg Results \\
\hline $\mathrm{SiO}_{2}$ & $0.95 \pm 0.03$ & 3.46 \\
$\mathrm{TiO}_{2}$ & $0.59 \pm 0.26$ & 0.09 \\
$\mathrm{Al}_{2} \mathrm{O}_{3}$ & $1.00 \pm 0.31$ & 0.75 \\
$\mathrm{Fe}_{2} \mathrm{O}_{3}$ & $0.97 \pm 0.13$ & 0.61 \\
$\mathrm{MnO}$ & $1.21 \pm 0.10$ & 0.07 \\
$\mathrm{MgO}$ & $1.29 \pm 0.08$ & 0.34 \\
$\mathrm{CaO}$ & Not analyzed in $100-$ mg aliquot \\
$\mathrm{Na}_{2} \mathrm{O}$ & $1.38 \pm 0.31$ & 0.18 \\
$\mathrm{~K}_{2} \mathrm{O}$ & $1.46 \pm 0.42$ & 0.13 \\
$\mathrm{P}_{2} \mathrm{O}$ & $0.80 \pm 0.17$ & 0.13 \\
\hline
\end{tabular}

${ }^{\mathrm{a}}$ The large discrepancies in $\mathrm{Na}, \mathrm{K}$, and $\mathrm{Mg}$ reflect the uncertainty in the sea-salt correction in samples with very small amounts of solid-phase $\mathrm{Na}, \mathrm{K}$, and $\mathrm{Mg}$ present.
TABLE 2

Average Analytical Differences Between Results of USGS Rock Standards as Blind Unknowns and Accepted Values, Using In-house Standards

\begin{tabular}{lcr}
\hline \multicolumn{1}{c}{ Oxide } & $\begin{array}{c}\text { Average Absolute } \\
\text { Difference for } \\
\text { 17 Analyses }\end{array}$ & $\begin{array}{c}\text { Average Value } \\
\text { of Oxide }\end{array}$ \\
\hline $\mathrm{SiO}_{2}$ & 1.02 & 63.66 \\
$\mathrm{TiO}_{2}$ & 0.04 & 0.89 \\
$\mathrm{Al}_{2} \mathrm{O}_{3}$ & 0.19 & 15.31 \\
$\mathrm{Fe}_{2} \mathrm{O}_{3}$ (total) & 0.12 & 6.01 \\
$\mathrm{MnO}$ & 0.02 & 0.11 \\
$\mathrm{MgO}$ & 0.08 & 1.93 \\
$\mathrm{CaO}$ & 0.09 & 3.96 \\
$\mathrm{Na} 2 \mathrm{O}$ & 0.10 & 3.32 \\
$\mathrm{~K}_{2} \mathrm{O}$ & 0.09 & 3.89 \\
$\mathrm{P}_{2} \mathrm{O}_{5}$ & 0.04 & 0.21 \\
\hline
\end{tabular}

by the Deep Sea Drilling Project. Joris Gieskes was most helpful in providing pore-water information in advance of their publication, and the DSDP loaned additional pore-water samples for determinations. The analyses were performed mainly by James Wallace, Daniel Li, Max Budd, and Carol Terrana, with some additional analyses by Gino Nalli, Laura Merrill, and T. Donnelly. We appreciate the assistance of F. J. Flanagan of the U.S.G.S. in arranging for the analysis of our in-house sediment standards by the U.S.G.S. Laboratory, which served as an important check for our own results.

\section{REFERENCES}

Donnelly, T. W., in press. Chemistry of sediments of the Western Atlantic: Site 417 compared with Sites 9, 105, 386, and 387. In Donnelly, T., Francheteau, J., Bryan, W., Robinson, P., Flower, M., Salisbury, M., et al., Initial Reports of the Deep Sea Drilling Project, v. 51, 52, 53, Part 1: Washington (U.S. Government Printing Office).

Donnelly, T. W. and Nalli, G., 1973. Mineralogy and chemistry of Caribbean sediments. In Edgar, N. T., Saunders, J. B., et al., Initial Reports of the Deep Sea Drilling Project, v. 15: Washington (U.S. Government Printing Office), p. 929-961.

Donnelly, T. W. and Wallace, J. L., 1976a. Major element chemistry of the Tertiary rocks at Site 317 and the problem of the origin of the nonbiogenic fraction of pelagic sediments. In Schlanger, S. O., Jackson, E. D., et al., Initial Reports of the Deep Sea Drilling Project, v. 33: Washington (U.S. Government Printing Office), p. 557-562. 1976b. Major- and minor-element chemistry of Antarctic clay-rich sediments: Sites 322, 323, and 325, DSDP Leg 35. In Hollister, C. D., Craddock, C., et al., Initial Reports of the Deep Sea Drilling Project, v. 35: Washington (U.S. Government Printing Office), p. 427-446. 
TABLE 3

Chemical Composition of Deep Sea Sediments, Sites 9 through 425, Deep Sea Drilling Project

SITE 9: LAT 32 DEG 46 MIA $\wedge$; LCNG 59 DEG LL MIN W; DEPIH 4SEL $N$ (ANAL BUDD)

SAMPLE DEPTH SIO2 TIU2 AL2C3 FE2O3 MNO MGO CAO NA2C
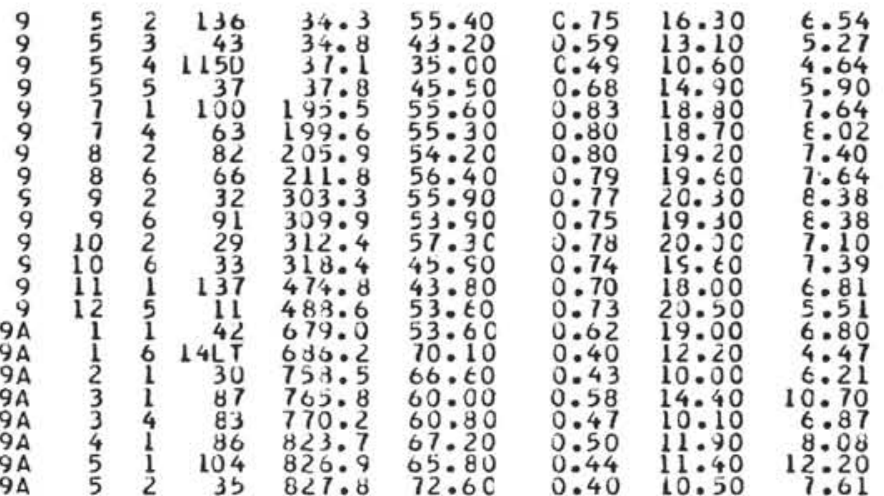

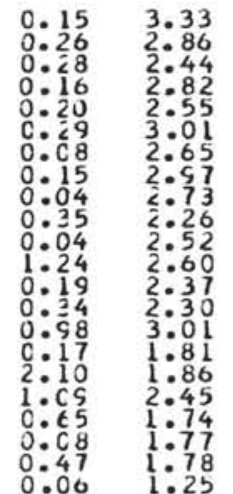
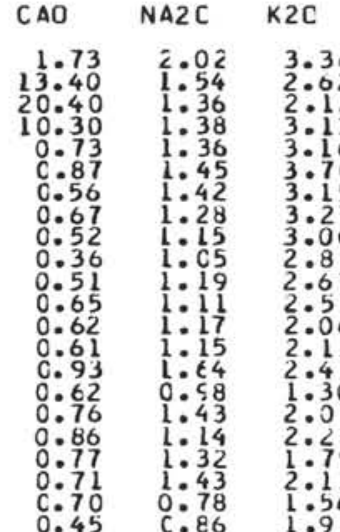

P205

$\mathrm{CL}$

LITHCLCGY

SITE 10:LAT 32 DEG $52 \mathrm{MIN} \mathrm{N}$; LCNG 52 DEG $13 \mathrm{MIN}$ W; DEPIH 4 E 12 M (ANAL WALLACE)

SAMPLE

$$
\text { DEPTH SIUZ }
$$

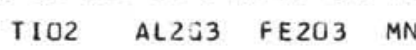

$M G O$

\section{$C$ A}

NA2O K 20
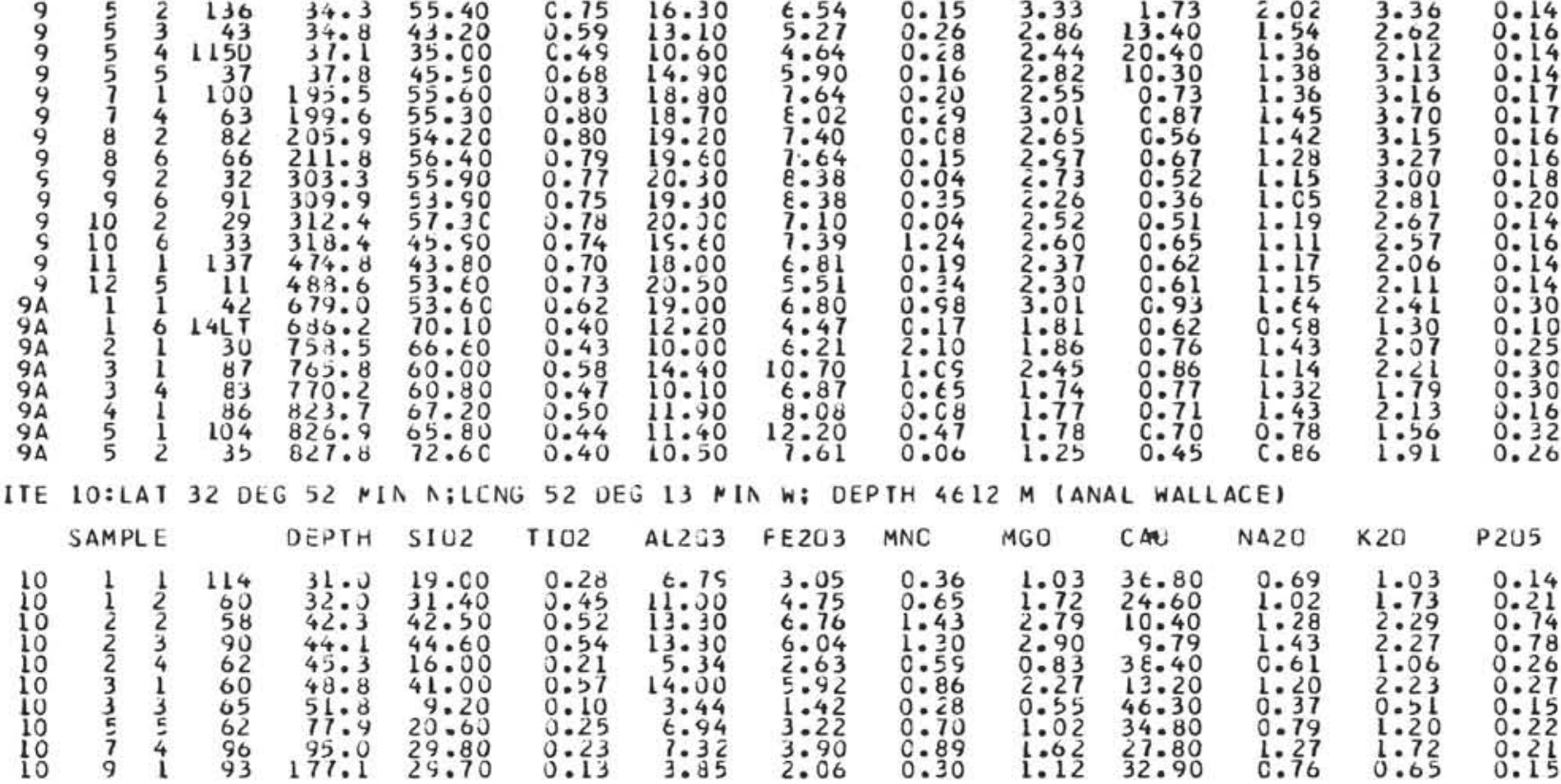

1.68

CALC. RAC. DOZE

$1: 74$ NANNO MARL

1.08

1.0

0.92 CLAY

0.68 CLAY

1.20 CLAY

0.49 CLAY

0.60 CLAY 0.32 ZECLITIC CLAY

0.94 RADICLARIAN COZE

0.68 LEGLITIC CLAY

0.63 ZEOLIIIC CLAY

P2U5 CL

CL

LI THCLOCY

SITE 14:LAT 2Q DEG 2J MIN S; LONG 20 CEG 56 NIN h;UEPTH 4346 M (ANAL hALLACE)

SAMPLE DEPTH SIC

IU2

MGO CAO NA2C
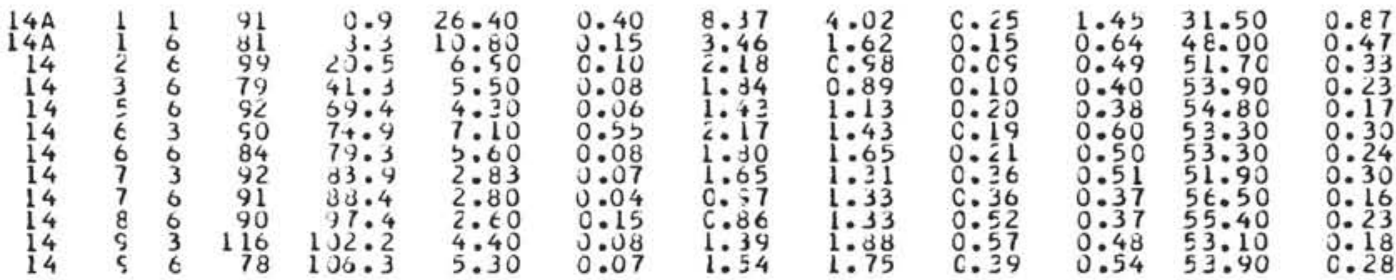

SITE 15:LAT $3 J$ UEG 53 MIN S: LCNG 17 DEG 59 NIN W; DEPTH 3638 MIANAL WALLACE) DEPTH SIU2 TIO2 AL2O3 FE2U3 MNC MGO

CAO

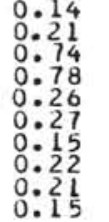

1.07
1.07
$2: 04$

$1: 79$

NANNC OOLE

1.25

CLAY

0.95

NANAC CCZE

SILIC. CAL
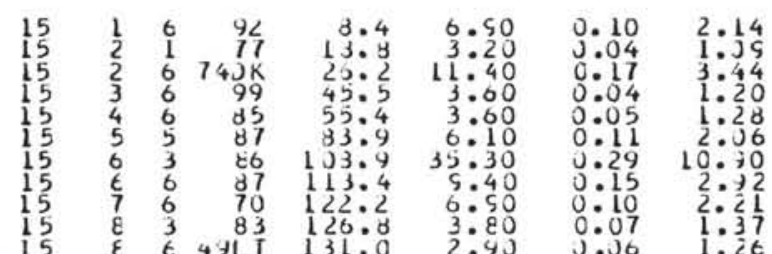

1.26
$0: 67$
$1: 63$
$0: 72$
$0: 69$
$1: 47$
$7: 11$
$2: 03$
$1: 68$
$2: 55$
0.55
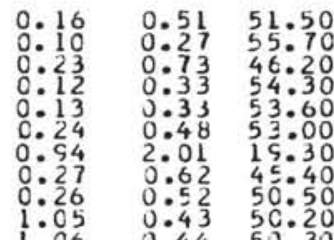

0.38
$0: 27$
$0: 54$
$0: 27$
$0: 17$
$0: 40$
$0: 99$
$0: 34$
$0: 25$
$0: 19$
0.14
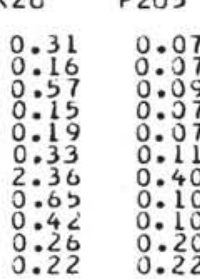

CL

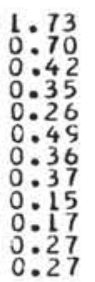

0.17
$0: 13$
$0: 08$
0.08
$0: 07$
$0: 07$
$0: 09$
$0: 09$
0.08
0.08

LITHCLCGY

1.00 NANAO CCZZE
0.87 NANNO OOZE

$0: 85$ NANN COZE

0.71 NANNO OCLE

0.92

. 77 NANNO LOZ

0.88 NANNO

0.10

0.77 NANNO CCZE

$\mathrm{CL}$

L I THCL UGY

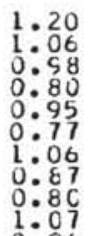

NANNO UOZE

NANNC COZE

NANAO CCLE

MARL COZE

0.87 AARA COLE

$0.8 C$ NANAC CCZE
1.07 NANAO CCZEE
0.94 NANNO CCZE 
SITE 16: LAT 3J DEC 2) MIN S; LCNG 15 JEG 43 NIN W; DEPTH 3526 M (ANAL WALLACE) SANPLE DEPTH SIUZ TIUZ ALZC3 FEZU3 MNO MGU CAU NA2C

P205

CL

LITHCLCGY
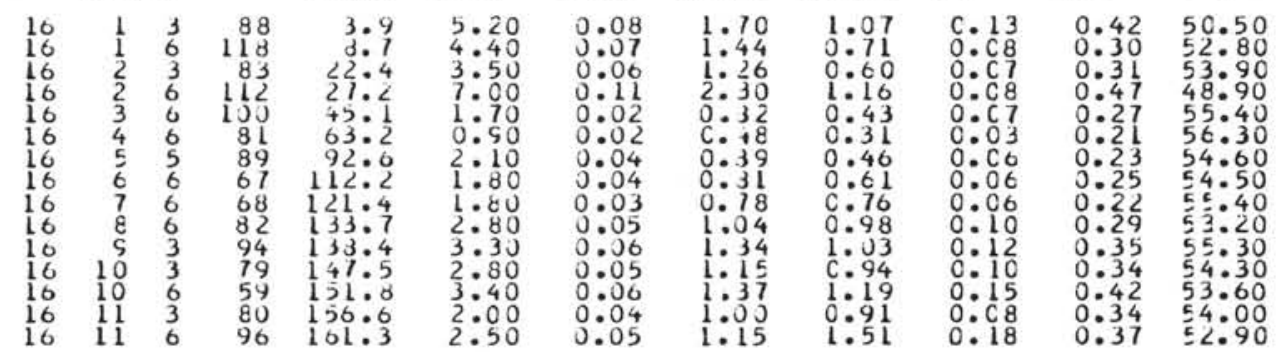

$0: 37$
$0: 20$
$0: 31$
$0: 39$
$0: 18$
$0: 14$
$0: 16$
$0: 24$
$0: 21$
$0: 20$
$0: 20$
$0: 21$
$0: 26$
$0: 10$
0.20

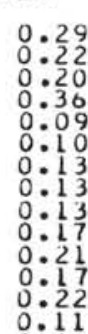

$\begin{aligned} & \text { SITE } \text { 1S: LAT } \\ & \text { SAMPLE }\end{aligned}$ DEPTH SIO2

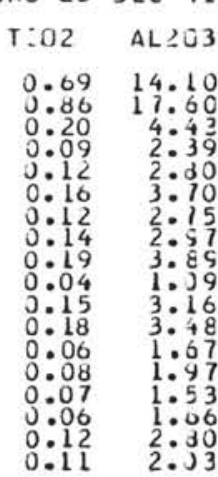

IN $h$ : DEPTH $4685 \mathrm{M}$ (ANAL WALLACE)

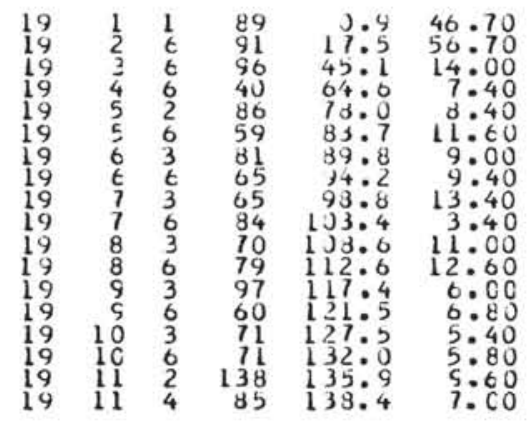

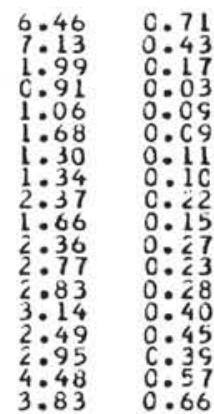

\begin{abstract}
MGO
\end{abstract}
$C A O$

NA2O K2

SITE 25: LAT O DEG 31 MIN S; LCNG 39 DEG 1+ MIN W; DEPTH $1916 \%$ (ANAL LI)

DEPTH SIC2

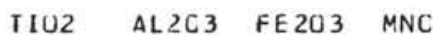

MGO

$\begin{array}{ll}2.42 & 10.40 \\ 3.08 & 1: 22 \\ 0.87 & 44.70 \\ 0.53 & 52: 40 \\ 0.65 & 50: 80 \\ 0.87 & 47.90 \\ 0.66 & 50.40 \\ 0.65 & 50.20 \\ 1.03 & 43.90 \\ 0.39 & 55.10 \\ 0.85 & 47.50 \\ 0.97 & 45: 30 \\ 0.60 & 51.90 \\ 0.67 & 50.00 \\ 0.55 & 52.90 \\ 0.61 & 52.90 \\ 0.91 & 45.50\end{array}$

1.80
1.56
0.49

0.49
0.29
0.52

0.42

0.28

0.15

0.39
0.43
0.28

0.28
0.29
0.30

0.30

SAMPLE
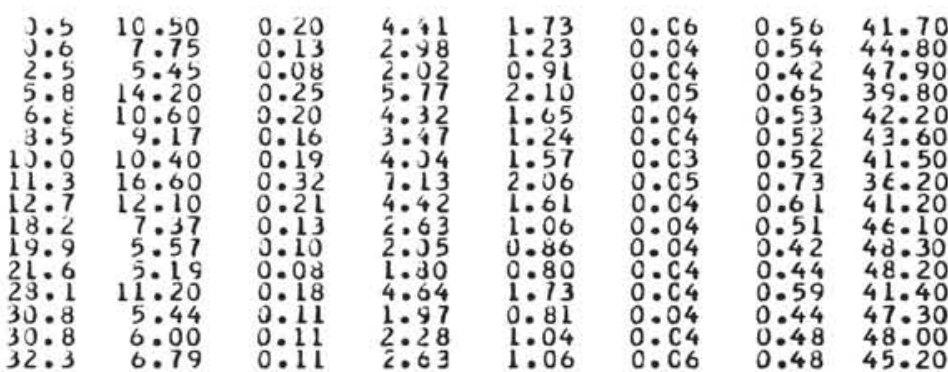

NA2C

2.65
$3: 66$
$1: 02$
$0: 54$
$0: 61$
$0: 83$
$0: 63$
$0: 67$
$0: 94$
$0: 28$
$0: 74$
$0: 87$
$0: 40$
$0: 46$
$0: 41$
$0: 41$
$0: 72$
0.50

P205

0.22

0.22
0.12
0.08

0.08

0.1 10

0.015

0.14

0.12

0.100

0.176

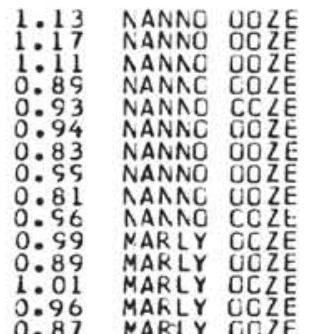

0.96 MARLY UCZE

CL LITHOLOGY

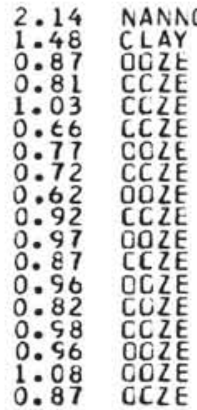

$\mathrm{CL}$

$0.53 \quad 0.08$

CL

LITHCLCGY

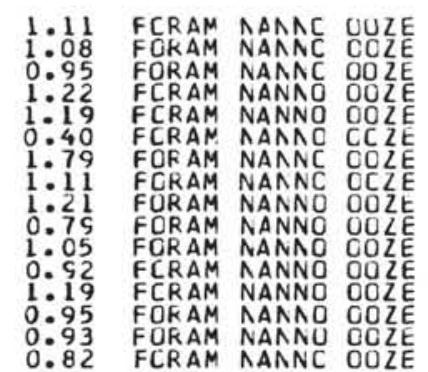

SITE 27: LAT IS DEG SI NIN $A$; LONG 56 DEG 33 MIN W; DEPTH 5253 M (ANAL. BLDC)

SAMPLE

DEPTH SIC2

$\begin{array}{cc}T I O 2 & A L 2 C 3 \\ 0.80 & 20.30 \\ 0: 78 & 13: 00 \\ 0: 83 & 20: 90 \\ 0: 84 & 21: 00 \\ 0: 92 & 22: 70 \\ 0.82 & 20: 10 \\ 0.86 & 21.00\end{array}$

$\begin{array}{ll}\text { FE2O3 } & \text { MNO } \\ 7.94 & 0.13 \\ 3.85 & 0: 09 \\ 8: 10 & 0.28 \\ 8.93 & 0: 12 \\ 6.07 & 0.10 \\ 7.08 & 0.41 \\ 6.68 & 0.09\end{array}$

\begin{abstract}
MGO
\end{abstract}

CAO

0.15

0.27

0.45

0.35

$0: 28$

0.26

$0.34 \quad 0.55$

0.32

0.3010 .05

$0.55 \quad 0.07$

.84

$0.67 \quad 0.08$

$\begin{array}{ll}.42 & 0.08 \\ .31 & 0.06\end{array}$

0.08

0.06

0.8

FORAM NAANU COZE

$\begin{array}{rrrrrr}27 A & 1 & 5 & 60 & 30.0 & 51.70 \\ 27 A & 2 & 3 & 77 & 49: 0 & 69: 50 \\ 27 A & 3 & 1 & 126 & 55: 0 & 52: 20 \\ 27 A & 4 & 3 & 109 & 67: 0 & 51: 80 \\ 27 A & 4 & 4 & 68 & 68: 0 & 50.60 \\ 27 A & 4 & 5 & 66 & 70: 0 & 50: 50 \\ 27 A & 5 & 2 & 47 & 74.0 & 50.70\end{array}$

NA2C K2O P205 CL

$\begin{array}{ll}1 . C 6 & \text { CLAY } \\ 0.38 & \text { TURBIDITE } \\ 1.20 & \text { CLAY } \\ 0.83 & \text { CLAY }\end{array}$

LITHCLCGY

0.03
$1: 87$
$1: 38$
$1: 28$

$.40 \quad 2.66$

0.16

0.13

$\begin{array}{lll}1: 42 & C L A Y \\ 0 & 82 & C L A Y \\ 1: 77 & \text { CLAY }\end{array}$ 


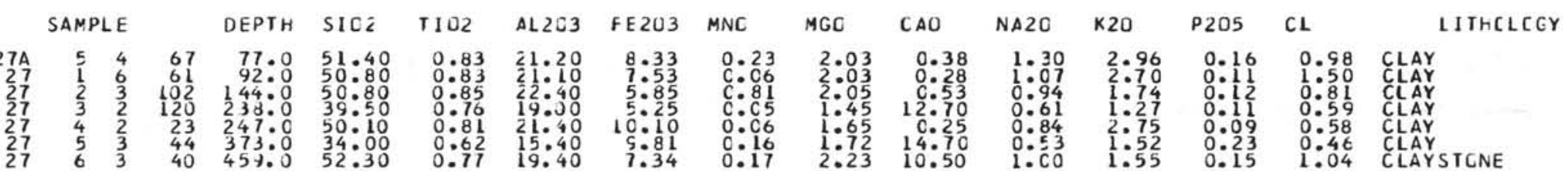

SITE 28: LAT 20 DEG 35 NIN N; LCNE 6S DEG 37 MIN W; DEPIH ES 19 M (ANAL. BLDD)

SAMPLE DEPTH SICÉ T102 ALZJ3 FE2U3 MNO MGO CAO NA2C K20 P205 CL L L

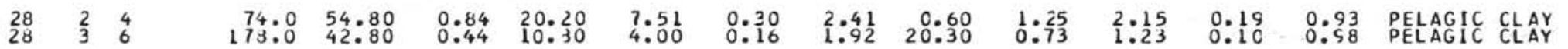

SITE 29: LAT 14 UEG 47 NIN $\wedge$; LCNG 69 UEG 19 MIN h; DEPTH 4247 M (ANAL. BUDD)

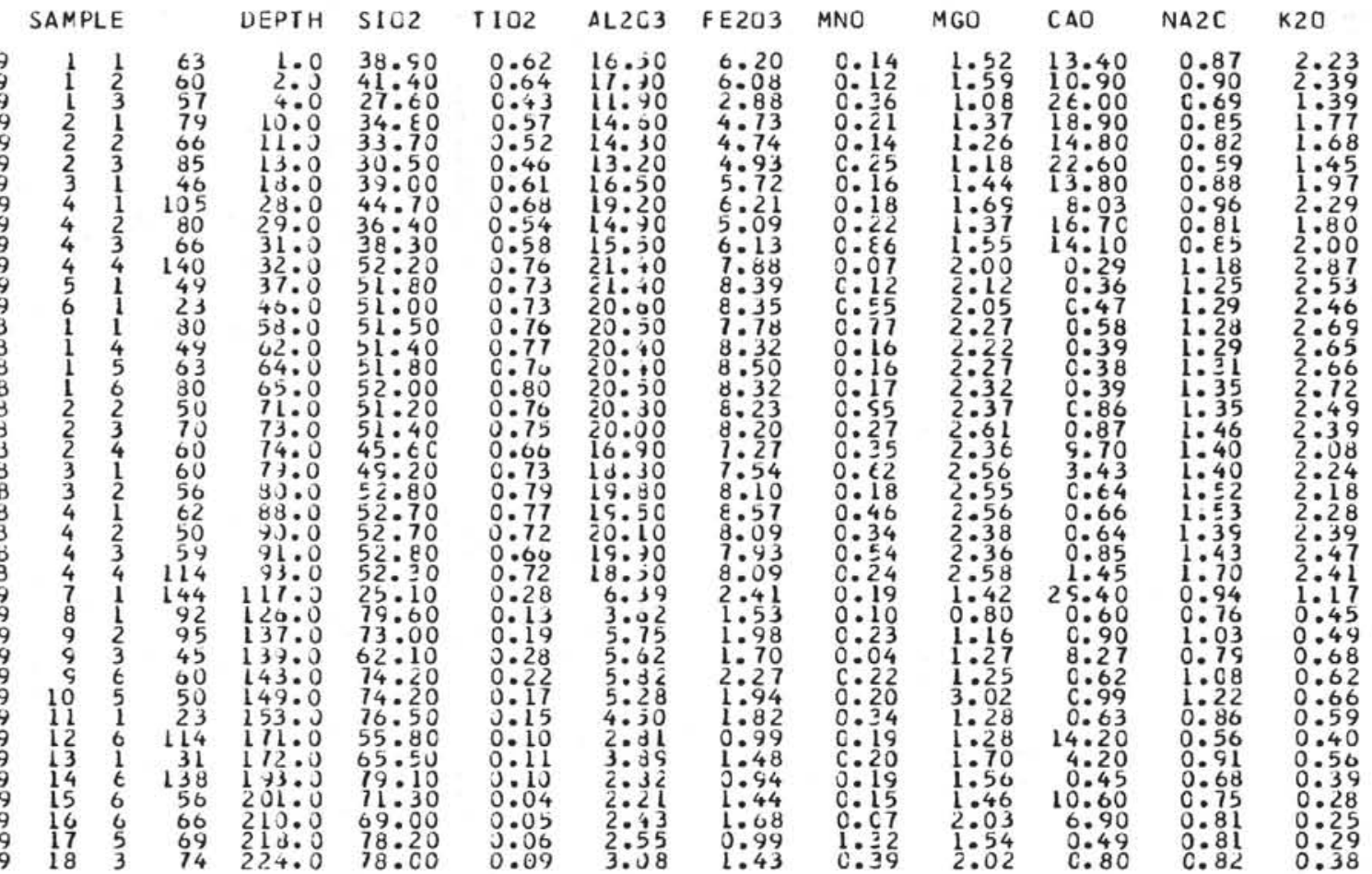

SITE 30: LAT 12 DEG 22 NIN A; LCNG 63 JEG 23 MIA n; DEPTH $1218 \mathrm{M}$ (ANAL. BUDD)

DEPTH SIUZ2

MGO

CAO NA2O
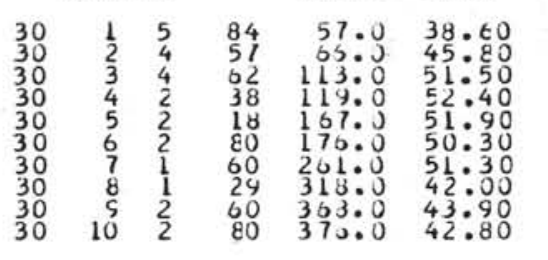

$\begin{array}{ll}0.61 & 15: 20 \\ 0: 76 & 10: 70 \\ 0: 85 & 19: 30 \\ 0.84 & 18: 10 \\ 0.63 & 17: 30 \\ 0: 88 & 20: 10 \\ 0: 91 & 19: 00 \\ 0.63 & 11: 10 \\ 0: 73 & 10: 100 \\ 0.61 & 13: 40\end{array}$

5.20
$7: 16$
6.68
6.33
6.30
$7: 37$
$6: 61$
$5: 19$
6.63
9.47

0.04
$0: 03$
$0: 05$
$0: 04$
$0: 05$
$0: C 4$
$0: 04$
$0: 04$
$0: 05$
0.04

$\begin{array}{rr}1.57 & 18.50 \\ 1.67 & 7.66 \\ 1.75 & 5.13 \\ 1.73 & 5.08 \\ 1.56 & 6.90 \\ 1.73 & 3.05 \\ 1.73 & 3.08 \\ 2.00 & 14.90 \\ 2.82 & 15.70 \\ 2.72 & 17.60\end{array}$

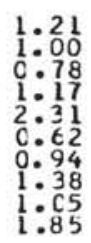

1.90
$2: 40$
2.52
2.26
$1: 80$
2.58
$2: 48$
$1: 97$
$1: 22$
1.58

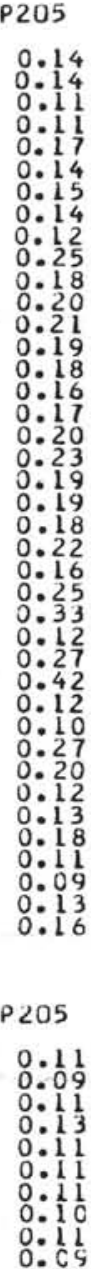

$\mathrm{CL}$

LITHCLCGY

$\begin{array}{llll}0.14 & 1.30 & \text { CALCAREOUS CLAY } \\ 0.14 & 1.20 & \text { CALCARECUS CLAY }\end{array}$

$0.111: 25$ CALCAREOLS CLAY

14 1.12 CALCAREOUS CLAY

0.14

0.25

11 CLAY

.00 CLAY

$: 04$ CLAY

1.03 CLAY

$1: 44$ CLAY

1.164 CLAY

$1: 04$ CLAY

1.23 CLAY

1.05 CLAY

0.94 CLAY

.96 CLAYEY CHALK

2.73 RADICLARIAN OOZE

3.02 RADICLARIAN OCZE

36 RAD

3.05 RADICLARIAN OOLE

2.72 RADIOLARIAN OCZE

3.33 RADICLARIAN OULE

2.85 RADICLARIAN COZE

$2: 54$
2.67
RADICLIARIAN DOZE

LI THOLCGY

$1: 63$ CALC: SHLTY CLAY

0.87 CALC: SILTY CLAY

1.26 CALC: SILYY CLAY

082 sily CLAY

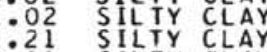




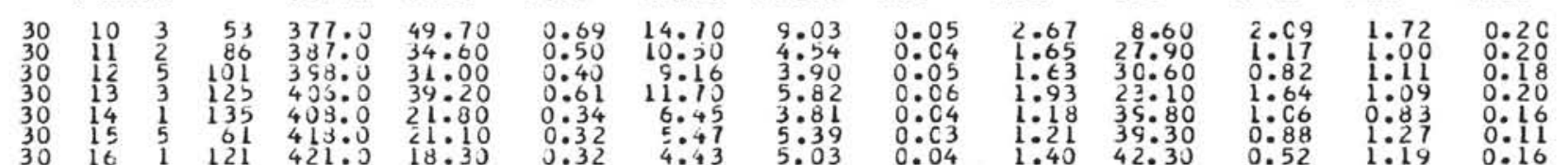

SITE 3L: LAT L4 DEG 57 NIA $N$ : LONG 72 DEG 1 MIN W; DEFTH 3363 M (ANAL. BULD)
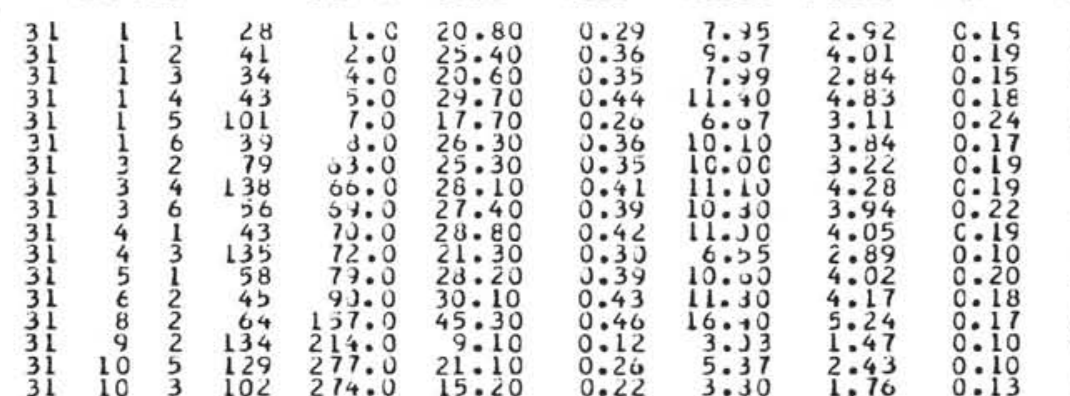

CAO NA2C

K20

P205 CL

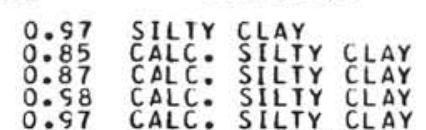

0.97
1.03
1.03 CALC: SILTY CLAY

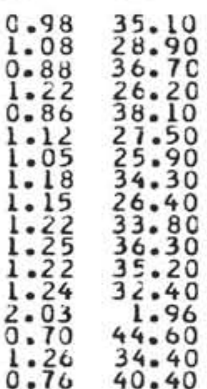

$0: 44$
$0: 38$
$0: 31$
$0: 50$
$0: 29$
$0: 43$
$0: 41$
$0: 44$
$0: 38$
$c: 44$
$0: 35$
$0: 42$
$0: 44$
$0: 78$
$0: 12$
$0: 39$

1.02
$1: 23$
104
$1: 45$
0.89
$1: 35$
$1: 28$
$1: 42$
1.46
0.68
1.52
1.63
2.26
0.42
.66

0.114

$\mathrm{CL}$

LITHOLCGY

SITE 34:LAT 3 S DEG 28 MIN N; LONG 127 JEG 17 MIN W:UEPTH $4 \equiv 22$ M (ANAL. LI) SAMPLE DEPIH SIO2

\begin{tabular}{|c|c|}
\hline 102 & $A L 203$ \\
\hline $\begin{array}{l}0.69 \\
0.72 \\
0.72 \\
0.69 \\
0.37 \\
0.44 \\
0.66 \\
0.69 \\
0.21 \\
0.64 \\
0.69 \\
0.70 \\
0.62 \\
0.61 \\
0.57 \\
0.60 \\
0.59 \\
0.62 \\
0.61 \\
0.52 \\
0.56 \\
0.66 \\
0.68 \\
0.63 \\
0.51 \\
0.67 \\
0.65 \\
0.53 \\
0.62 \\
0.44 \\
0.60 \\
0.63 \\
0.63 \\
0.71 \\
0.72 \\
0.37 \\
0.85 \\
0.66 \\
0.49\end{array}$ & 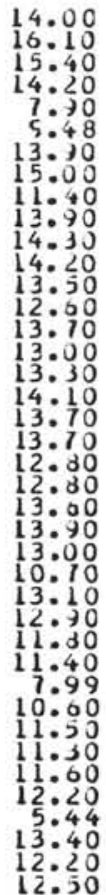 \\
\hline
\end{tabular}

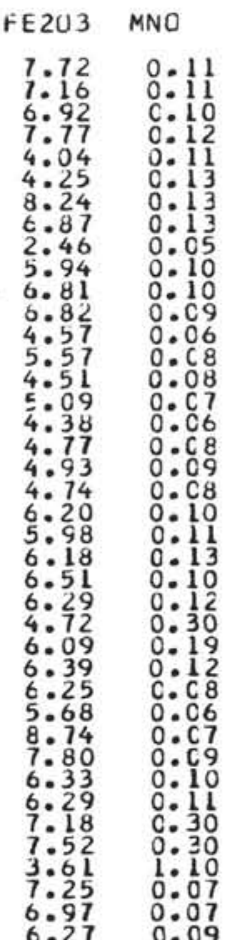
MGO $C A O$ NA2O K2O
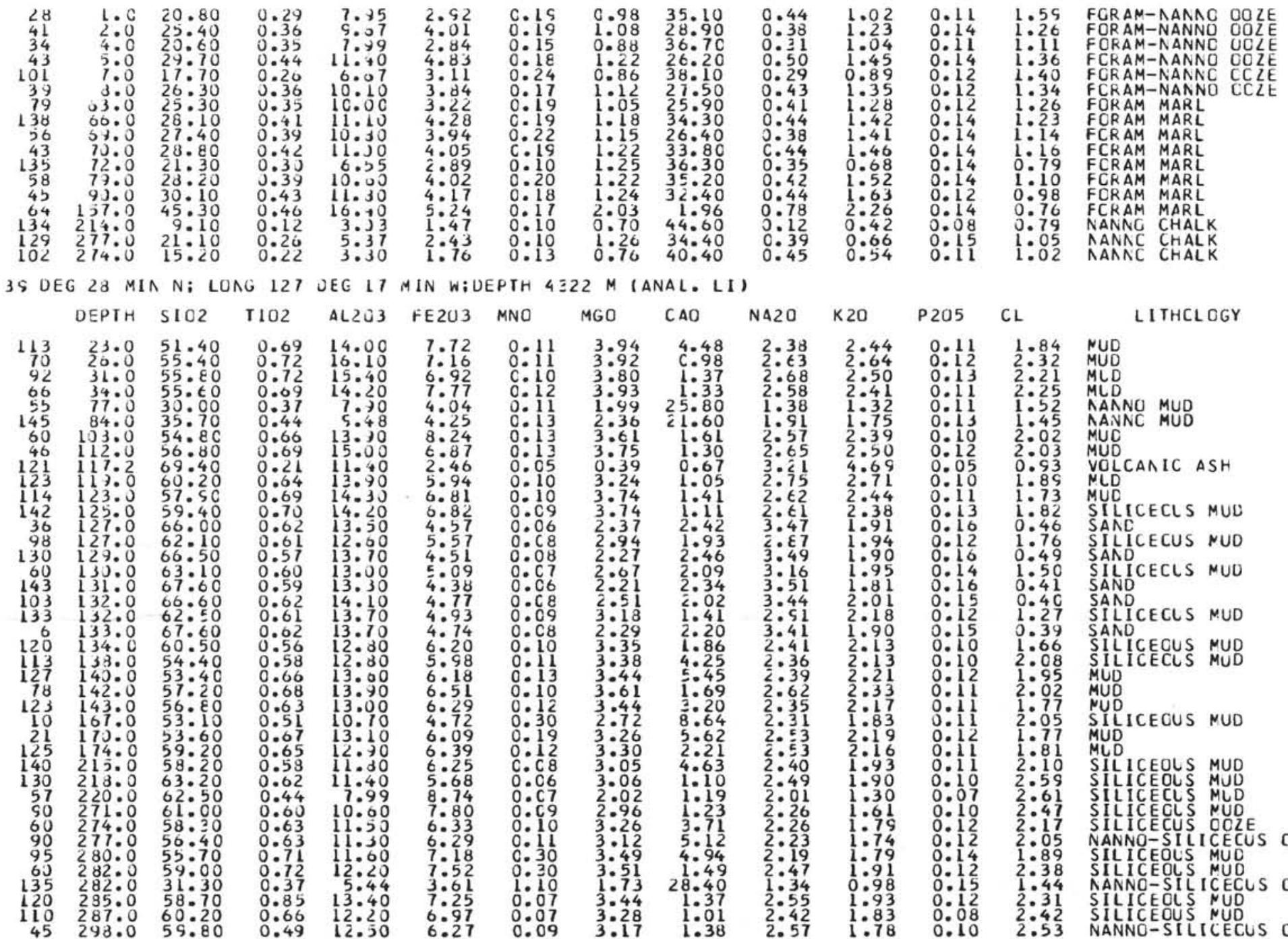

0.4912 .50

$\begin{array}{llll}6.27 & 0.07 & 3.28 & 1.09\end{array}$

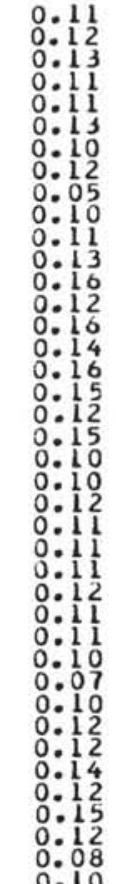

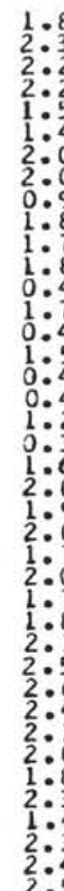

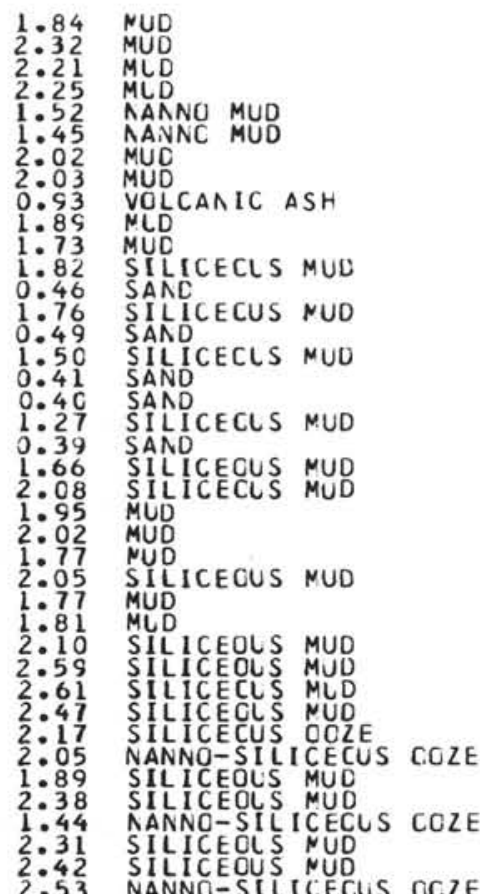




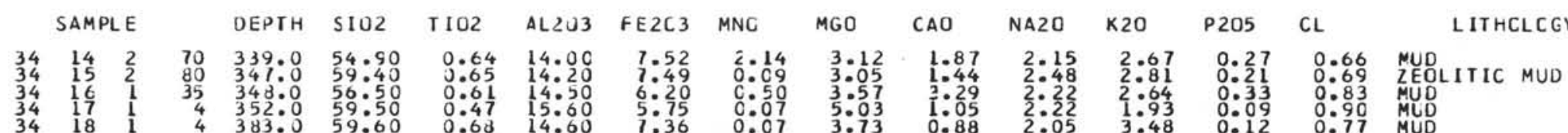
SITE 37:LAT 40 UEG 59 MIN N: LCNG 140 UEG $43.1 \mathrm{~N}$ h; DEPIT $4682 \mathrm{M}$ (ANAL BUCD)

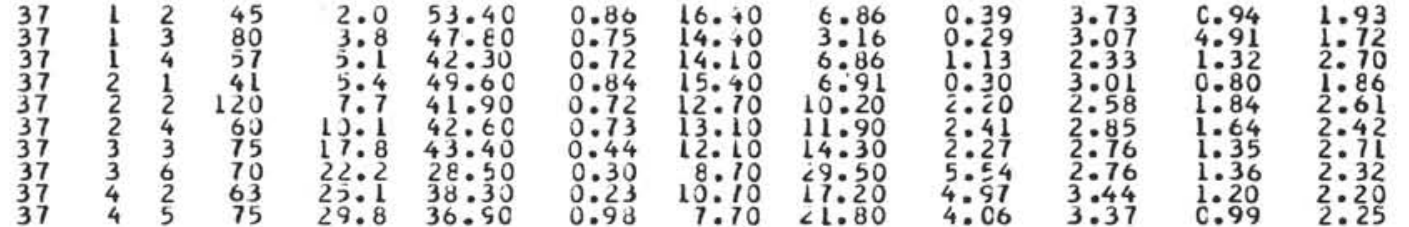

SItE 42: LAT 13 DEG $5 L$ NIA $\wedge$; LCNG 140 CEG 12 MIN W; CEFIH 4848 M (ANAL WALLACE) SANPLE DEPIH SIC2 TIU2 AL2U3 FE203 MNC MGO CAO NA2C
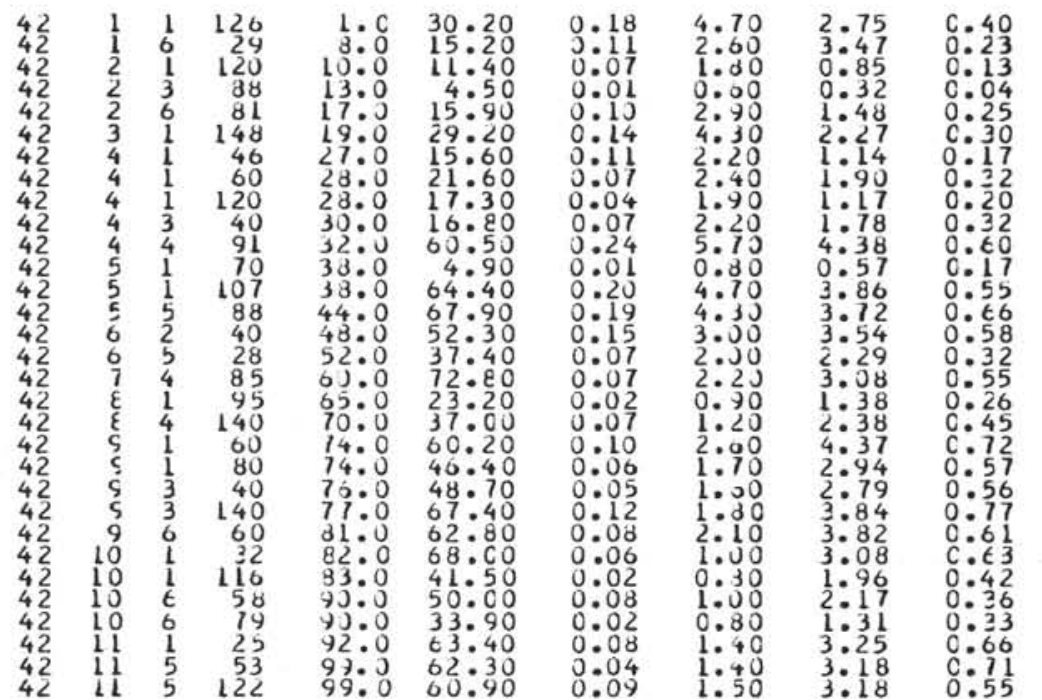

$\begin{array}{lll}1.47 & 27.60 & 0.94 \\ 0.82 & 44: 60 & 0.68\end{array}$

0.94
$0: 68$
$0: 45$
0
0
018

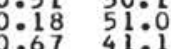

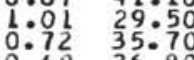

0.56 $\begin{array}{lll}0.68 & 36.80 & 0.65 \\ 0.45 & 39.30 & 0.48 \\ 0 & 40 & 40\end{array}$ $\begin{array}{lll}0: 61 & 3 \varepsilon: 50 & 0: 52 \\ 1: 74 & 5: 15 & 1: 23 \\ 0: 0 & 45: 40 & 0: 0\end{array}$

$\begin{array}{llll}51 & 1: 43 & 9: 14 & 0: 57 \\ 0.22 & 0: 88\end{array}$ SANPLE

JEG 36 IIN E; DEPTH 2591 M(ANAL. WALLACE)

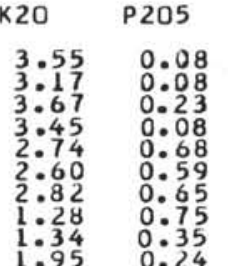

$\mathrm{CL}$

LITHOLCGY

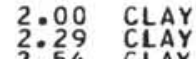
2:54 CLAY 3.61 ZEEOLIIIC CLAY 3.83 FERUGINCLS CLAY 4:86 FERRUGINOLS CLAY

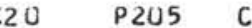
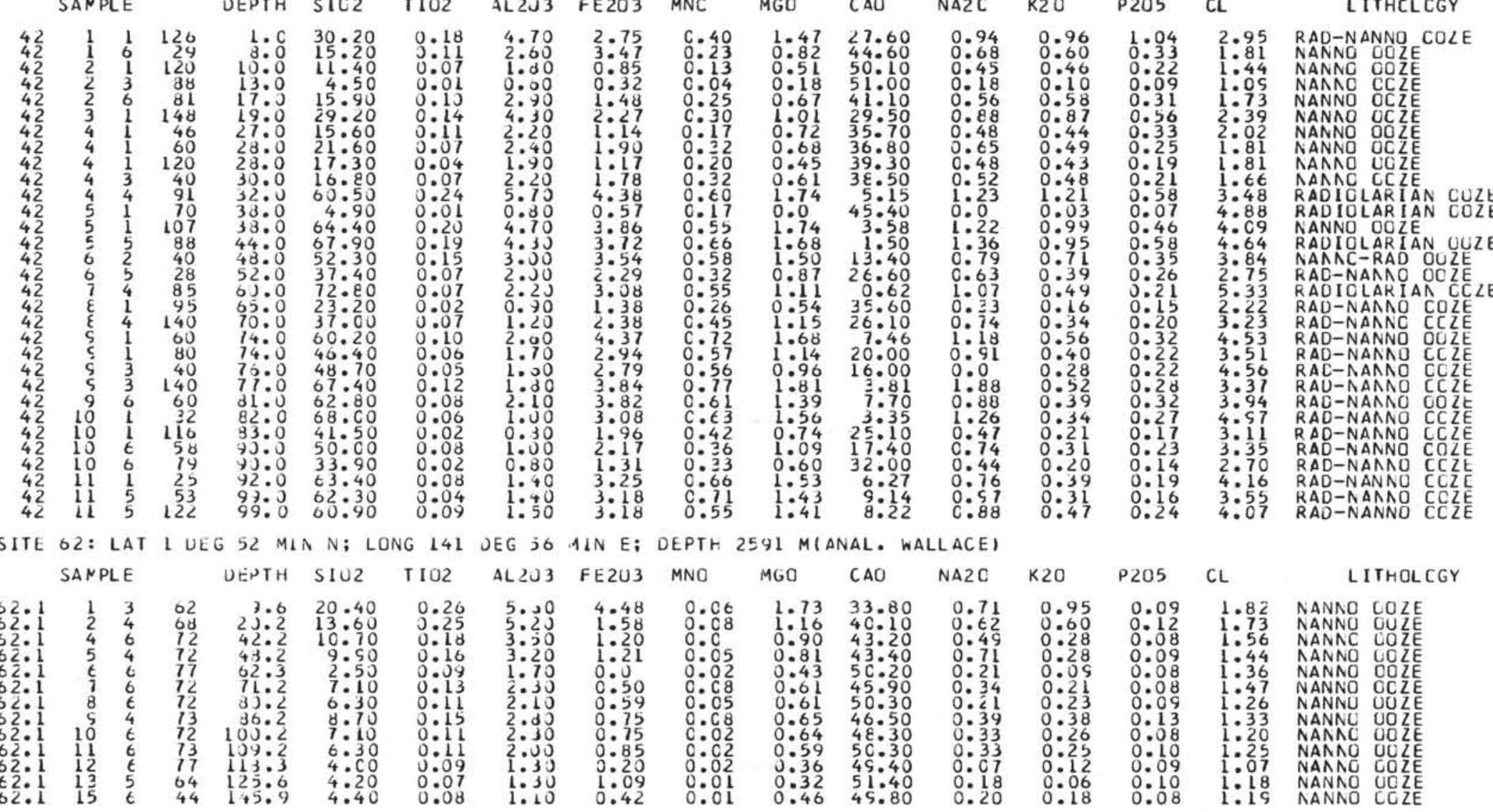
MGO

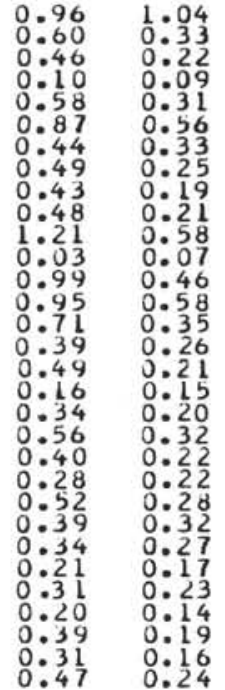

4.55

RAD-NANAO CCZE
RAD-NANNO CCZE
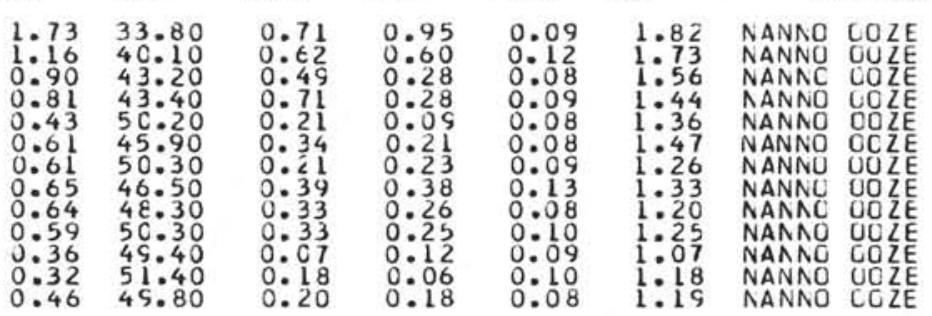


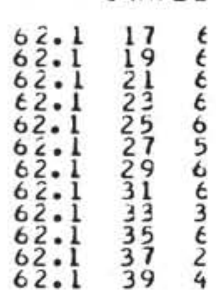

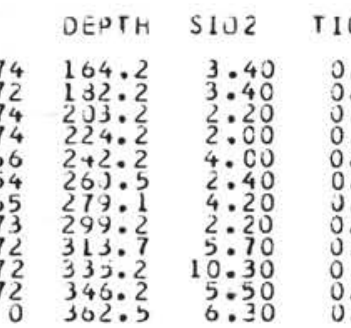

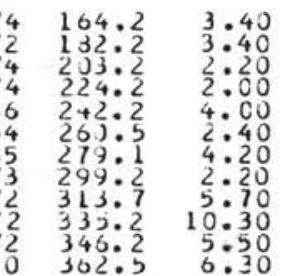

ALLJ3 FELO3 MNC MGO

$C A O$

NA2O K2O

P 205

$\mathrm{CL}$

LITHCLCGY

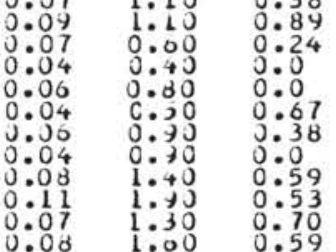

0.0
$0: 0$
$0: 0$
$0: 0$
$0: 0$
$0: 0$
$0: 0$

0.19
$0: 16$
$0: 02$
0.20

0.05
$0: 12$
$0: 06$
0.01
0.13

0.13

0.09
0.09
0.08

1.18 NANNO COZE
1.06 NANNO OOZE

0.06

0.07

0.08

$\begin{array}{llll}0.0 & 0.32 & 54.10 & 0.124 \\ 0.05 & 0.63 & 49.20 & 0.24\end{array}$

SITE EE: LAT

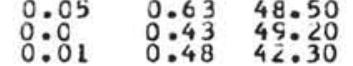

0.16

SAMPLE

$24 \mathrm{NIN} N$;

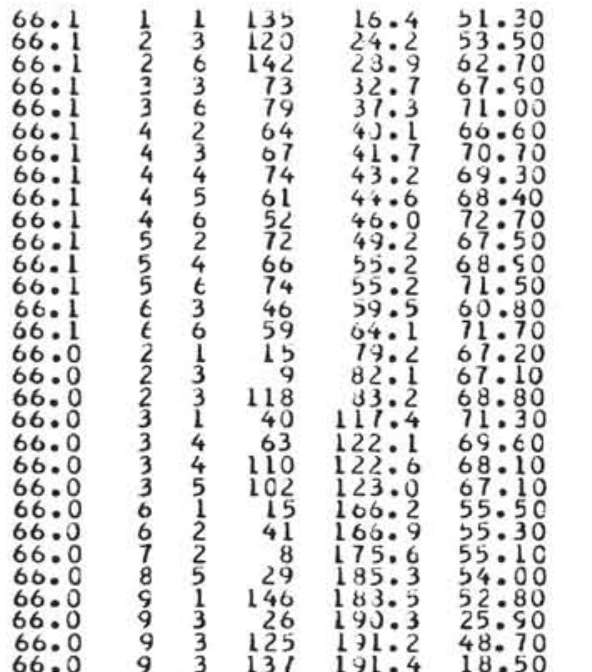

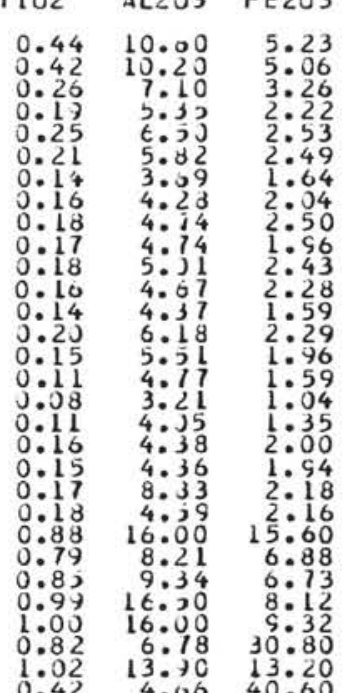

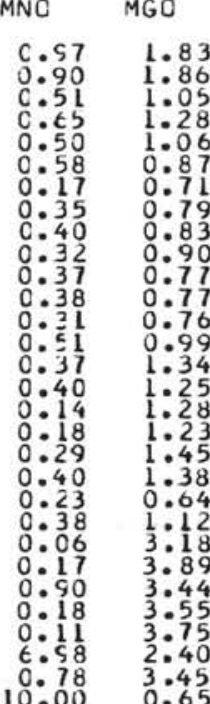

CAO
1.09
$1: 05$
$0: 55$
$0: 44$
$0: 78$
$0: 91$
$0: 72$
$0: 68$
$0: 58$
$0: 66$
$0: 63$
$0: 58$
$0: 62$
$0: 92$
$0: 56$
$0: 71$
$4: 04$
$1: 63$
$1: 05$
$1: 20$
$1: 47$
$1: 46$
$0: 46$
$0: 25$
$0: 51$
$0: 42$
$0: 35$
$3: 06$
1.56
$2: 07$

NA2O

K20 P205 CL

$\mathrm{CL}$

SITE 70: LAT O UEG 23 MIN N; LCNG 14J JEG 22 MIN W; DEPTH 5059 M (ANAL. hALLACE)

SAMPL DEPIH SIO2 TIO2 AL2J3 FE2O3 MNC

MGO

NA2O

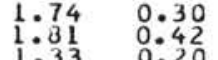

6.

1.16 NANNC DOZE

: II NANNO OOZE

16 NANNO CHALK

0.93 NANNO CHALK

$\begin{array}{ll}1.33 & 0.20 \\ 1.08 & 0.17 \\ 1.41 & 0.21\end{array}$

1.07
0.74

0.52

0.30

0.94

0.85
$0: 99$
0.97

$0.97-0.28$

0.28

0.27
$1: 52$
0.92

0.92

$\begin{array}{ll}3: 44 & 0.64 \\ 45 & 078 \\ 15 & 074\end{array}$

0.42

0.26

0.60

$0.82 \quad 0: 85$

0.71

4.64

0.34

0

$\begin{array}{ll}3: 97 & 0.075 \\ 1 & 1: 28\end{array}$

.99 RaDiclarian cuze

5:76 RADICLARIAN COZE

6.25 RADICLARIAN OCZ

5.84 Radiclarian CCLE

5035 RADIOLARIAA UCZE

$\begin{array}{ll}4: 68 & R A D I O L A R I A N \\ 5: 50 & R A D I O L A R I A N \\ 5\end{array}$

5 Radiclarian UOzE

$7: 02$ RADICLARIAN OCZZE

$6: 58$ RADICLARIAN COL

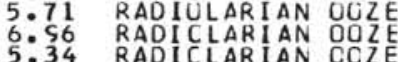

5.24 RADICLARIAN CCZE

5:39 RADICLARIAN CCL

$1: 70$ BROWN CLAY

1.38 BRCWN CLAY

1.69 BROWN CLAY

3.34
$1: 75$
1

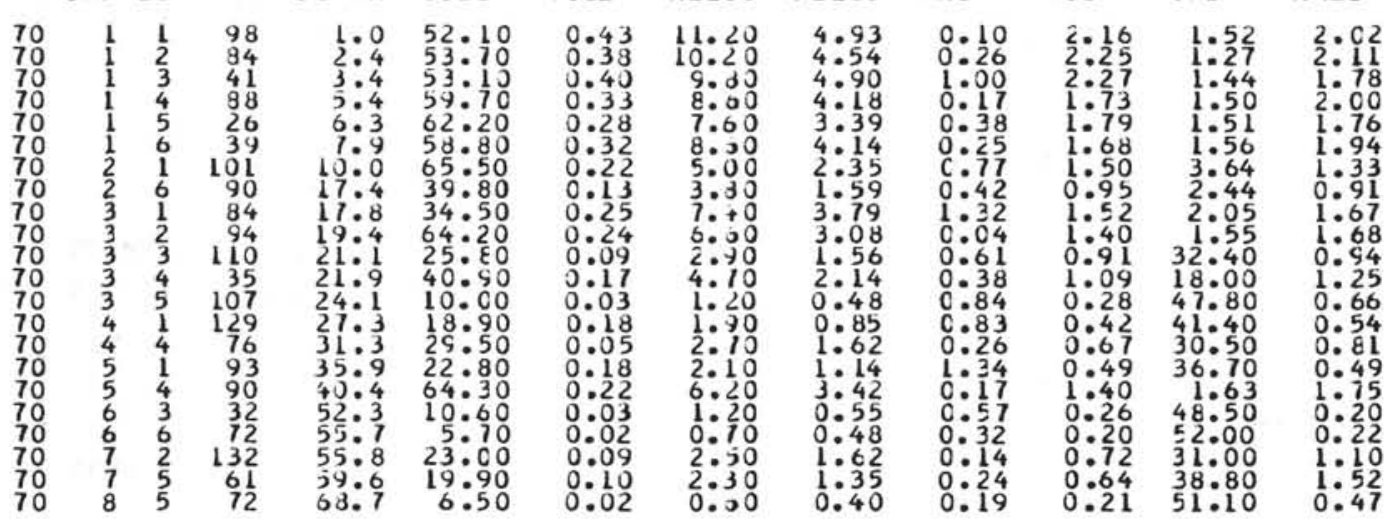

$20 \quad P 205 \quad C$

LIIHOLCGY

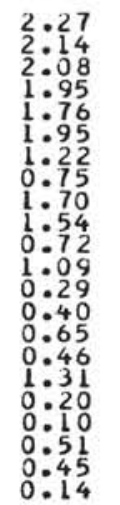

0.41

$0: 44$

0.52

0.26

0.33

$0: 33$

$0: 14$

$0: \frac{16}{31}$

0.20

0.15

0.28

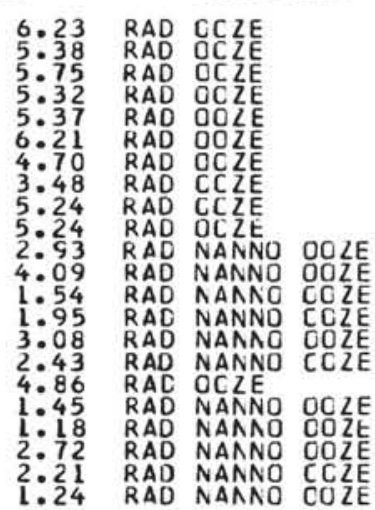




\begin{tabular}{|c|c|c|c|c|c|c|c|c|c|c|c|c|c|}
\hline \multicolumn{4}{|c|}{ SANFLE } & DEPTH & SICz & T IOL2 & $A L 203$ & FE 203 & MNO & MGO & CAO & NA2O & $k 20$ \\
\hline $\begin{array}{l}70 \\
70 \\
70 A \\
70 A \\
70 A \\
70 A \\
70 A \\
70 A \\
70 A \\
70 A \\
70 A \\
70 A \\
70 A \\
70 A \\
70 A \\
70 A \\
70 A \\
70 A \\
70 A \\
70 A\end{array}$ & $\begin{array}{r}10 \\
12 \\
1 \\
\frac{3}{3} \\
4 \\
5 \\
7 \\
9 \\
11 \\
13 \\
15 \\
16 \\
18 \\
18 \\
19 \\
21 \\
22 \\
25 \\
27 \\
27\end{array}$ & $\begin{array}{l}5 \\
5 \\
6 \\
6 \\
4 \\
6 \\
5 \\
3 \\
3 \\
4 \\
4 \\
2 \\
1 \\
2 \\
3 \\
1 \\
3 \\
3 \\
1 \\
3\end{array}$ & $\begin{array}{r}92 \\
71 \\
42 \\
92 \\
54 \\
100 \\
5 \\
21 \\
90 \\
114 \\
23 \\
135 \\
110 \\
54 \\
33 \\
112 \\
38 \\
106 \\
116 \\
23\end{array}$ & $\begin{array}{r}87: 9 \\
110: 7 \\
120: 9 \\
139: 4 \\
145: 0 \\
157: 5 \\
174: 1 \\
189: 2 \\
204: 9 \\
224: 6 \\
242: 7 \\
251: 4 \\
263: 1 \\
264: 0 \\
272: 4 \\
282: 1 \\
290: 4 \\
309: 1 \\
321: 2 \\
323: 2\end{array}$ & $\begin{array}{r}8: 20 \\
24: 80 \\
18: 20 \\
28: 20 \\
15: 30 \\
4: 15 \\
5: 64 \\
3: 60 \\
3: 40 \\
4: 10 \\
11: 00 \\
4: 10 \\
74: 80 \\
32: 50 \\
29: 40 \\
19: 00 \\
11: 00 \\
12: 10 \\
12: 00 \\
66: 20\end{array}$ & $\begin{array}{l}0: 02 \\
0: 09 \\
0: 05 \\
0: 12 \\
0: 06 \\
0: 03 \\
0: 04 \\
0: 04 \\
0: 03 \\
0: 03 \\
0: 05 \\
0: 03 \\
0: 12 \\
0: 11 \\
0: 04 \\
0: 05 \\
0: 04 \\
0: 04 \\
0: 03 \\
0: 16\end{array}$ & $\begin{array}{l}1.00 \\
2: 40 \\
1: 30 \\
2: 70 \\
1: 30 \\
0: 40 \\
0: 70 \\
0:+00 \\
0: 400 \\
0: 30 \\
1: 00 \\
0: 40 \\
3: 00 \\
2: 80 \\
0: 900 \\
0: 70 \\
0: 30 \\
0: 70 \\
0: 30 \\
3: 90\end{array}$ & $\begin{array}{l}0: 47 \\
1: 95 \\
0: 71 \\
2: 39 \\
1: 06 \\
0: 16 \\
0: 63 \\
0: 50 \\
0: 44 \\
0: 20 \\
0: 73 \\
0: 40 \\
2: 67 \\
2: 62 \\
1: 01 \\
0: 30 \\
0: 92 \\
0.40 \\
1: 35 \\
5: 25\end{array}$ & $\begin{array}{l}0: 70 \\
0: 09 \\
0: 15 \\
0: 09 \\
0: 04 \\
0: 14 \\
0: 12 \\
0: 06 \\
0: 03 \\
0: 08 \\
0: 15 \\
0: 09 \\
0: 34 \\
0: 16 \\
0: 11 \\
0: 12 \\
0: 15 \\
0: 11 \\
0: 27 \\
0: 93\end{array}$ & $\begin{array}{l}0: 21 \\
0: 65 \\
0: 36 \\
0: 97 \\
0: 45 \\
0: 17 \\
0: 32 \\
0: 21 \\
0: 19 \\
0: 22 \\
0: 36 \\
0: 19 \\
1: 56 \\
1: 16 \\
0: 40 \\
0: 31 \\
0: 43 \\
0: 22 \\
0: 27 \\
2: 26\end{array}$ & $\begin{array}{l}48: 60 \\
32: 00 \\
42: 20 \\
32: 10 \\
45: 80 \\
52: 20 \\
49: 80 \\
50: 00 \\
51: 10 \\
55: 40 \\
44: 50 \\
56: 00 \\
1: 74 \\
25: 80 \\
34: 50 \\
42: 70 \\
49: 10 \\
52: 10 \\
42: 50 \\
1: 86\end{array}$ & $\begin{array}{l}0: 32 \\
0: t 4 \\
0: 54 \\
0: 92 \\
0: 36 \\
0: 18 \\
0: 26 \\
0: 11 \\
0: 09 \\
0: 19 \\
0: 34 \\
0: 18 \\
2: 14 \\
1: 03 \\
0: 43 \\
0: 29 \\
0: 24 \\
0: 24 \\
0: 22 \\
1: 30\end{array}$ & $\begin{array}{l}0.15 \\
0: 53 \\
0: 31 \\
0: 60 \\
0: 22 \\
0: 13 \\
0: 17 \\
0: 16 \\
0: 11 \\
0: 04 \\
0: 24 \\
0: 14 \\
0: 42 \\
0: 48 \\
0: 27 \\
0: 22 \\
0: 16 \\
0: 10 \\
0: 11 \\
0: 64\end{array}$ \\
\hline
\end{tabular}

P205 CL

LITHCLCGY

ITE 73: LAT 1 DEG 55 MIN S; LCAG 137 DEG 28 NIN N; CEPTH 4387 M (ANAL hALLACE)

SAMPLE

DEPIH SIL2 T1O2 AL2J3 FE2U3 MNC

$\begin{array}{lll}73 & 1 & 2 \\ 73 & 2 & 4 \\ 73 & 3 & 3 \\ 73 & 4 & 4 \\ 73 & 5 & 6 \\ 73 & 6 & 2 \\ 73 & 6 & 4 \\ 73 & 7 & 3 \\ 73 & 7 & 6 \\ 73 & 8 & 3 \\ 73 & 8 & 6 \\ 73 & 9 & 3 \\ 73 & 9 & 6 \\ 73 & 10 & 4 \\ 73 & 11 & 4 \\ 73 & 12 & 6 \\ 73 & 13 & 2 \\ 73 & 14 & 4 \\ 73 & 15 & 5 \\ 73 & 16 & 6 \\ 73 & 17 & \\ 73 & 18 & \\ 73 & 19 & \\ 73 & 19 & \\ 73 & 20 & 4 \\ 73 & 20 & 5 \\ 73 & 21 & \end{array}$

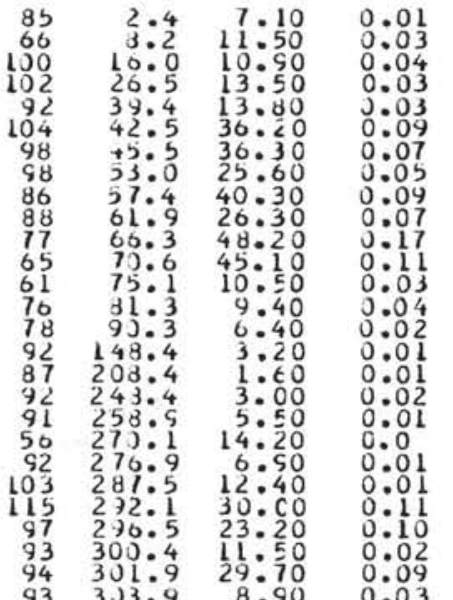

CAO

A2C

K20
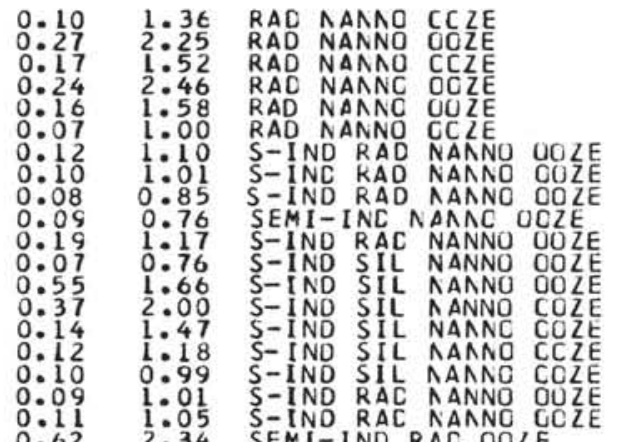

1.05 S-IND RAC NANNO GC

P205 CL

LITHCLOGY

$0.06 \quad 1.86$ SIL FCRAM/AANNO COZE

1.81 SIL FORAM/NANNO COZE
$2: 35$ SIL FCRAMINANNO CCZE
0.31 SIL FCRANINANNO COZE

2.07 SIL FCRAMINANNO COZE

3.24 RAD NANNO COLE

3.49 RAD NANNO DOZE

2.11 RAD NANAO CCZE

3.93
3.89
$3 A D I O L A K I A N ~ O O L E$

1.68 RAD NANNO COZE

$1: 25$ RAD NANNO CCZE

0.90
$0: 65$ NANNO OOZE

0.73 NANAO UCZE

0.90 NANAC CCZE

0.82 NANND OCLE
0.86 SILICEOUS NANNO UCZE

SIL ICEOUS NANNO GCZZE

1.09
$0: 58$

KAD NAANO CEZE

$0 . t 4$ RAD NANAC CCZ

$\begin{array}{lllllll}1.70 & 2: 78 & 0.37 & 0.89 & 33.50 & 0.46 & 0: 45 \\ 0.70 & 1.15 & 0: 25 & 0: 78 & 31: 40 & 0: 39 & 0: 4 \\ 1: 70 & \frac{1}{3}: 45 & 0: 12 & 0.42 & 50.60 & 0: 15 & 0: 1\end{array}$

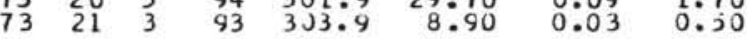

$\begin{array}{llllll}3: 95 & 0: 55 & 1: 08 & 33: 30 & 0: 76 & 0: 52 \\ 3.33 & 0.51 & 1.22 & 46.90 & 0.31 & 0.39\end{array}$

0.18

SITE 75: LAT

DEPTH SIE2 TIOZ

ALLJ3 $+E 2 O 3$ MNU

MGO

CAL WALLACE)

$\begin{array}{lll}75 & 1 & 1 \\ 75 & 1 & 2 \\ 75 & 1 & 3 \\ 75 & 1 & 4 \\ 75 & 1 & 5 \\ 75 & 1 & 5 \\ 75 & 2 & 2 \\ 75 & 2 & 4 \\ 75 & 2 & 6 \\ 75 & 3 & 3 \\ 75 & 3 & 6 \\ 75 & 4 & 3 \\ 75 & 4 & 6 \\ 75 & 5 & 2 \\ 75 & 5 & 4 \\ 75 & 5 & 6 \\ 75 & 7 & 4 \\ 75 & 8 & \end{array}$

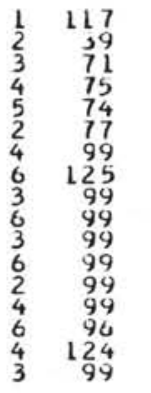

$1: 2$
$1: 9$
$3: 7$
$5: 3$
$6: 7$
$11: 3$
$14: 5$
$17: 8$
$22: 0$
$26: 5$
$31: 0$
$35: 5$
$33: 5$
$41: 5$
$44: 5$
607
$33: 0$

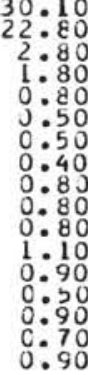

$\begin{array}{lll}0.30 & 5.10 & 20.00 \\ 0.25 & 5: 10 & 21: 100\end{array}$

$4: \varepsilon 4$

NA2O K2O

P205

$\mathrm{CL}$

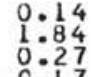

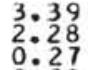

LITHOLCGY

5.19
4.38 ZECLITIC REC CLAY

$1: 32$ AANNO OCZE WITH CLAY

0.96 NANA OOZE

0.93 NANNO COZE

0.75 NANNO UCZE

0.78 NANNO CCZE
0.83 NAANC OCZE

0.80 NANAO CCZE

0.73 NANAN CCZE

0.91 NANAC COZE

0.92
0.96
$0 A A N O C$ CCZE 


\begin{tabular}{|c|c|c|c|c|c|c|c|c|c|c|c|c|c|}
\hline \multicolumn{4}{|c|}{ SAMPLE } & DEPIF & SIUZ & 102 & $A L 2 J 3$ & FE203 & MN J & $M G O$ & CAU & NA2C & K20 \\
\hline $\begin{array}{l}75 \\
75 \\
75 \\
75 \\
75 \\
75\end{array}$ & $\begin{array}{l}8 \\
9 \\
9 \\
9 \\
9 \\
9 \\
9\end{array}$ & $\begin{array}{l}\epsilon \\
1 \\
2 \\
3 \\
4 \\
5 \\
6\end{array}$ & $\begin{array}{r}94 \\
92 \\
109 \\
65 \\
25 \\
82 \\
18\end{array}$ & $\begin{array}{l}72: 5 \\
73: 9 \\
73: 0 \\
75: 6 \\
7708 \\
73: 8 \\
30.7\end{array}$ & $\begin{array}{l}0: 50 \\
0: 70 \\
0: 80 \\
0: 50 \\
0: 90 \\
0: 90 \\
1: 10\end{array}$ & $\begin{array}{l}0.0 \\
0: 01 \\
0: 01 \\
0: 02 \\
0.02 \\
0: 02 \\
0.02\end{array}$ & $\begin{array}{l}0 . J 2 \\
0: 08 \\
0: 25 \\
0: 10 \\
0: 25 \\
0: 24 \\
0: 25\end{array}$ & $\begin{array}{l}1.57 \\
0: 67 \\
4: 99 \\
2: 83 \\
4: 82 \\
3: 29 \\
5: 30\end{array}$ & $\begin{array}{l}0.46 \\
c: 14 \\
2: 09 \\
0: 86 \\
2: 11 \\
1: 11 \\
2: 41\end{array}$ & $\begin{array}{l}0: 14 \\
0: 16 \\
0: 27 \\
0: 18 \\
0: 31 \\
0: 20 \\
0: 33\end{array}$ & $\begin{array}{l}4 \epsilon .80 \\
54: 40 \\
50.20 \\
43: 10 \\
51: 00 \\
54: 60 \\
42: 70\end{array}$ & $\begin{array}{l}0: 15 \\
0: 10 \\
0: 13 \\
0: 03 \\
0: 31 \\
0: 03 \\
0: 14\end{array}$ & $\begin{array}{l}0.05 \\
0: 07 \\
0: 04 \\
0: 07 \\
0: 100 \\
0: 07 \\
0.13\end{array}$ \\
\hline
\end{tabular}

SITE 83: LAT + DEG 3 MIN $\wedge$; LCAG 95 OEj 4+ MI 4 i DEPTH 3646 M (ANAL WALLACE)

$$
\text { SAMPLE }
$$

$$
\text { DEPTH SICZ }
$$

$$
1102
$$$$
\text { AL2J3 FE203 MNC }
$$$$
\text { MGC }
$$$$
\text { CAO }
$$$$
\begin{array}{rrl}
83 & 1 & 4 \\
83 & 2 & 6 \\
83 A & 2 & 6 \\
83 A & 3 & 6 \\
83 A & 4 & 6 \\
83 A & 5 & 6 \\
83 A & 6 & 6 \\
83 A & 7 & 6 \\
83 A & 8 & 6 \\
83 A & 5 & 6 \\
83 A & 10 & 6 \\
83 A & 11 & 6 \\
83 A & 12 & 6 \\
83 & 5 & 6 \\
83 A & 14 & 6 \\
83 A & 15 & 6 \\
83 & 6 & 6 \\
83 A & 16 & 6
\end{array}
$$$$
\begin{array}{lll}
75 & 5.6 & 12.90 \\
75 & 13: 4 & 18: 50 \\
75 & 33: 4 & 20: 60 \\
75 & 33: 6 & 18: 90 \\
75 & 43: 8 & 24: 50 \\
90 & 33: 0 & 30: 20 \\
80 & 67: 1 & 40.60 \\
80 & 15: 2 & 43.30 \\
70 & 35: 3 & 34: 10 \\
75 & 94: 4 & 13: 20 \\
40 & 103: 3 & 29: 10 \\
80 & 112: 8 & 22: 70 \\
75 & 121: 9 & 36: 50 \\
75 & 144: 4 & 29: 20 \\
74 & 166: 3 & 22: 70 \\
75 & 187: 7 & 32: 10 \\
75 & 210.6 & 10: 20 \\
75 & 219 & 0
\end{array}
$$$$
\begin{array}{ll}
0.11 & 2.30 \\
0.11 & 2.40 \\
0.17 & 3.20
\end{array}
$$$$
0.12 \quad 2: 30
$$$$
.194 .05
$$$$
0.28 \quad 5.30
$$

SITE 84:LAT 45 MIN $\mathrm{A}$; $\varepsilon 2$

\section{NIN hi}

0.20
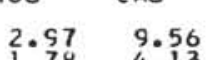

NA2 C

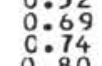
0.80 $1: 33$ 0.63 0.34 0.20

SITE 92; LAT 25 DEG 51 NIN $\wedge$; LCNG 92 DEG 49 MIN W; DEPTH 2573 M; (ANAL. LI)
SAMFLE
DEPTH SIC 2
T 102
AL $2 \mathrm{~J}_{3}$ FE2O3 MNC
MGO

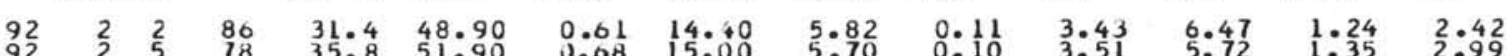

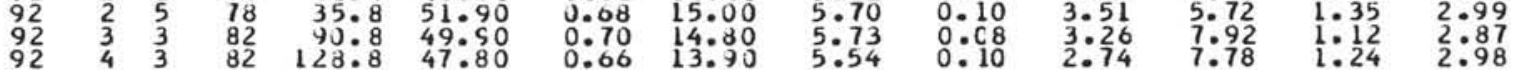

0.07

0.06

0.17

0.22

0.27

0.29

0

0.15

$0: 11$

0.15

$\mathrm{CL}$

FARAUGLCZE NANO UOZE FERRUE: NANAC CCZE

$\mathrm{CL}$

LITHCLCGY

2.93 CYCLIC HECDEC COZE 4.77
4.50 CYCLIC BEDDEC OCZE 4.56 CYCLIC BECCED ODLE 6.40 CYCLIC BEDDEU SOZE 6.03 CYCLIC BEDCEC OUZE (1) $2: 75$ $3: 19$ CYCLIC BEDDED UUZE 2.34 $2: 04$ CYCLIC EEDOED UOZE
$3: 31$ FERRGINCLS CHALK

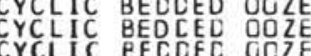

$205 \mathrm{CL}$ 0.12 ${ }_{0}^{0}: 13$ $0_{0}^{0}: 14$ 0.15 $0: 14$ $0: 17$ $0: 12$ 0
0
$0: 13$
$0: 16$ 0.16 $0: 11$

LITHCLCGY

3.27 RAD-CLAY-AANNO OOZE 3.43 RAD-CLAY-AANNO OUZE
$3: 17$ RAD-CLAY-NAANO COZE 3.02 $3: 77$
$3: 109$ OLCANIC ASH 3. OS RALCALAY-NANNO OOZE 0.63 VCLCANIC ASH $3: 28$ RAD-CLAY-NANNO DOZE $2: 92$ RAD-CLAY-NANNO COZE $\begin{array}{ll}1.89 & R A D-C L A Y-N A N N C \\ 2.71 & R A D-C L A Y-N A N A C \\ 2 & \text { OCZE }\end{array}$ 2.79 RAD-CLAY-NANNO DOLE $1: 65$ RAD-CLAY-NANAC DOZE 2.35
1.65 1.56 RAD-CLAY-NANNO OCZE 1.53
1.77
$1.7 A D-C L A Y-N A N N C$
RAD-CLAY-NANNO OOZE 1.77
2.51
$2 A D-C L A Y-N A N N O$
1

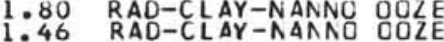
$1: 46$
$1: 12$ RAD-CLAY-AANNO CCZE
1.12 RAD-CLAY-NANAO CCZE P205 CL LITHCLCGY

$0: 17 \quad 0: 94$ SILTY CLAY $\begin{array}{lll}0: 20 & 0: 86 & \text { SILTY CLAY } \\ 0.22 & 0: 90 & \text { SILTY CLAY }\end{array}$ 


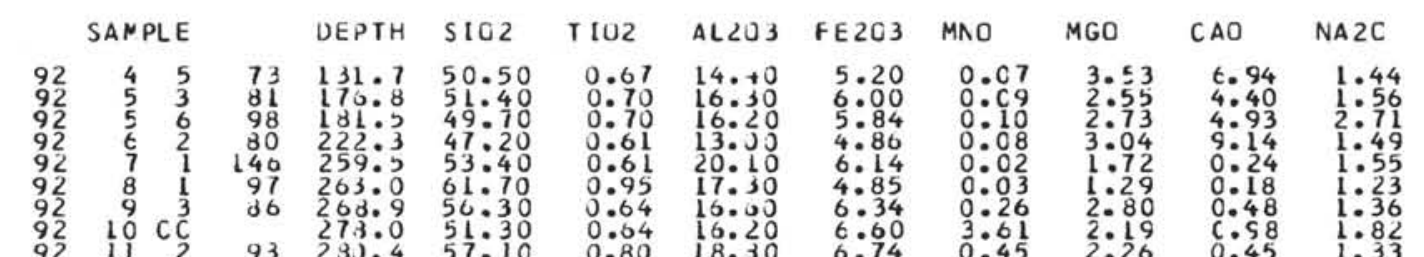

SITE S4: LAT 24 DEG 32 MIN N; LCNG 88 JEG 28 MIN W; DEPTH. 1793 M(ANAL TERRANA)

SAMPLE

$$
\text { DEPTH SIC2 }
$$

94
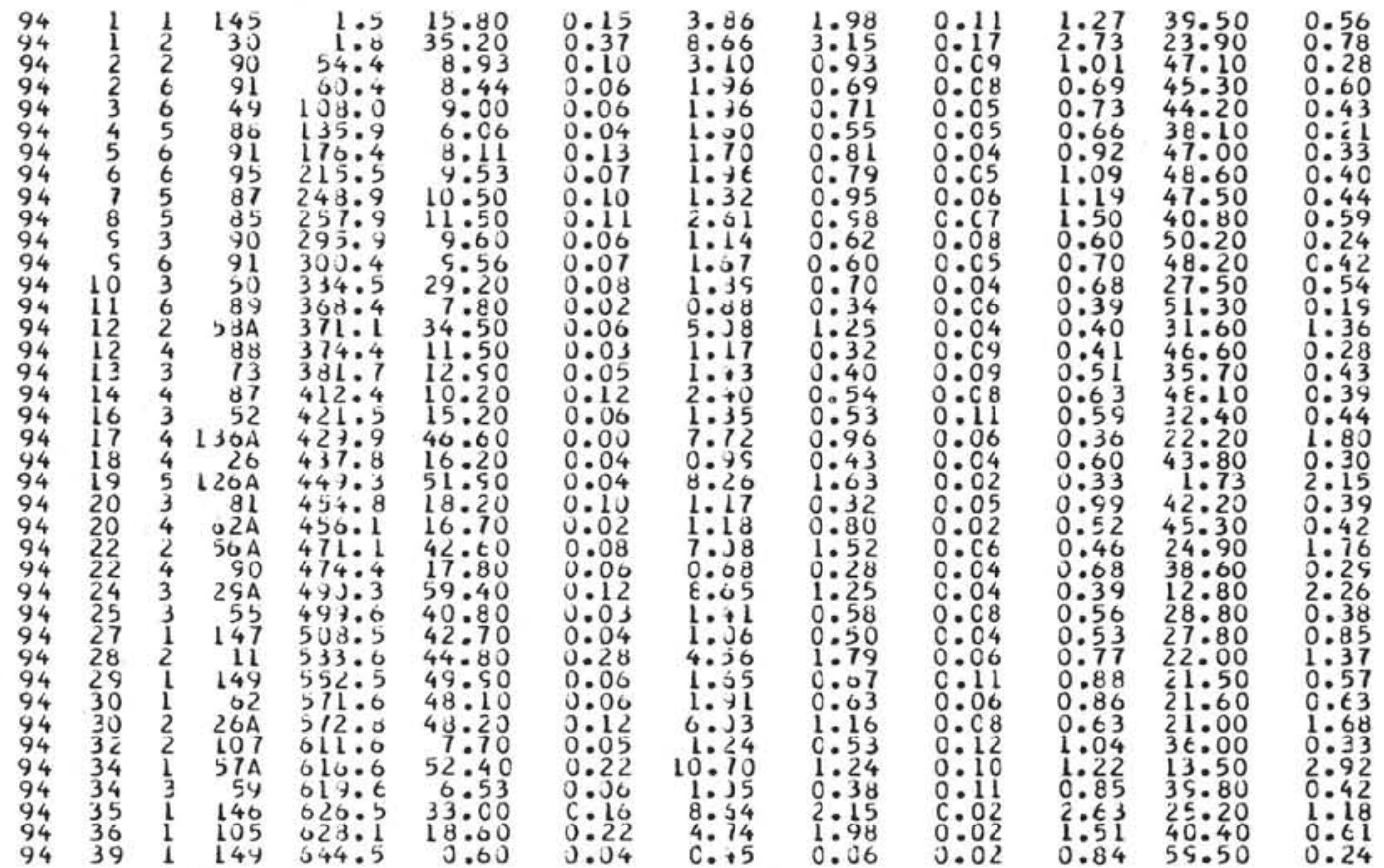

0.7364 .2

$0.92 \quad 470$

$\begin{array}{ll}1: 09 & 48: 60 \\ 1: 50 & 47: 50 \\ 1: 50 & 40.80 \\ 0.70 & 58: 20\end{array}$

0.6

.70 19.20 0.24

0.68 2. $27.50 \quad 0.54$

0.40113 .60

$\begin{array}{lll}0: 51 & 35: 70 & 0: 43 \\ 0.63 & 4 \varepsilon: 10 & 0\end{array}$

0.36 22:40 $1: 44$

$\begin{array}{lll}0: 60 & 43: 80 & 0 \\ 0 & : 30 \\ 03 & 1: 73 & 2: 15\end{array}$

$0: 99242: 20 \quad 00: 39$

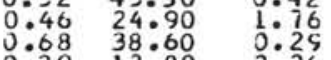

$.39 \quad 12: 80$

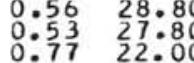

0.88 21:50 $1: 37$ 0.86 2 21.600 .63 $\begin{array}{lll}1: 04 & 3 \epsilon: 00 & 0: 33 \\ 0: 22 & 13: 50 & 2: 92 \\ 0.85 & 35: 80 & 0: 42\end{array}$

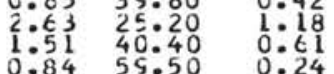

SITE 98: LAT 25 UEG 3 MIN N; LCNG 17 JEG 1 MIN W; UEPTH $2 I E 9$ N (ANAL hALLACE)

$$
\text { SANPLE }
$$$$
\begin{array}{lll}
98 & 1 & 1 \\
98 & 1 & 5 \\
98 & 2 & 3 \\
98 & 2 & 6 \\
98 & 3 & 3 \\
98 & 3 & 6 \\
98 & 4 & 3 \\
98 & 4 & 6 \\
98 & 5 & 2 \\
98 & 5 & 4 \\
98 & 6 & 2 \\
98 & 6 & 5 \\
98 & 8 & 3 \\
98 & 9 & 2 \\
98 & 10 & 1 \\
98 & 11 & 2
\end{array}
$$

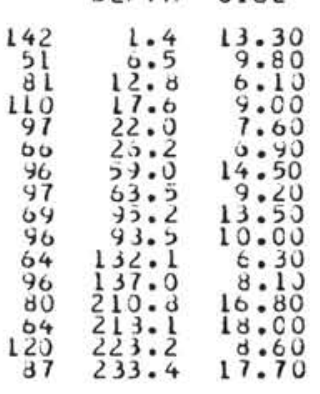

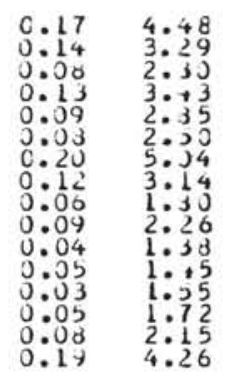

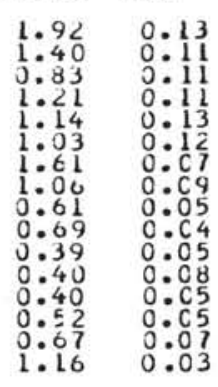

MG

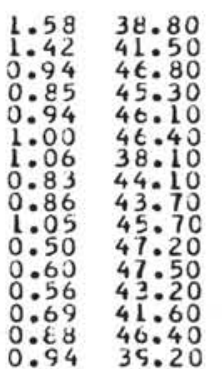

$\begin{array}{ll}K 20 & \text { P205 } \\ 2.91 & 0.17 \\ 2.86 & 0: 17 \\ 2: 38 & 0: 18 \\ 2.42 & 0: 22 \\ 2.41 & 0: 17 \\ 1.95 & 0: 09 \\ 2.65 & 0: 17 \\ 2.65 & 0.17\end{array}$

K20

${ }_{1}^{0}: 62$

$0: 41$
$0: 29$
$0: 27$
$0: 30$

0.25
$0: 30$
$0: 33$

0

$0.34 \quad 0.18$

0.1950 .16

$0: 28$ 0. 0

$0: 27 \quad 0: 18$

$\begin{array}{ll}2: 78 & 0: 04 \\ 0: 20 & 0: 11 \\ 3: 18 & 0: 04\end{array}$

$0.20 \quad 0: 10$

$2.55 \quad 0.04$

$2: 96 \quad 0: 06$

$0: 28 \quad 0: 08$

$0.35 \quad 0: 10$

$1: 95 \quad 0: 06$

$3: 17$
$0: 37$

$1: 04$
$1: 24$
0.08

$0: 14$

0.18

NA2C

0.62

00.31

0.29

0.42

$0: 29$

0.18

$0 .: 42$

0
0.42
0.44
K20 P205 CL

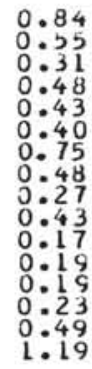

0.12
LITHCLCGY

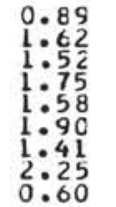

SILTY CLAY

SILTY CLAY

, MUDSICNE

CLAYSTONE MUUSTONE CLAYSICNE, MUDSTONE

\section{$\mathrm{CL}$ \\ LITHCLCGY \\ 1:16 FCRAM-AANNO OOZE

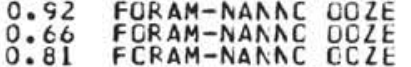 0.86 FCRAM-AANAC DCZE 0.71 FCRAM-NAANC DOZE
0.53 FCKAM-NANNO OCLE $0: 67$ FOKAM-NANAC DOZE 0.90 FCRAM-NANNO OCZE FO 7 FCRAM-NANNO OCZE
0.70 FCRAM-NANNO OCZE 9 FORAM-NANNC UCL GCRAN-NAANO UOZE
0.70
0.98 FOKAM-NANNO COZZE 0.55
0.53 FCRAN-AANAC COZE 0.70 VULCANICAASH
$0: 60$ FCRAM-NANNU CHALK .83 Volcanic ash CHalK :06 VCLCANIC AST ALE 0.71 VCLCANIC ASH

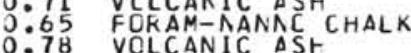 1033 FORAM-AAND CHALK 1.00 FORAM-NANNC CHALK 0.87 FCRAM-AANAC CHALK
0.94 FCRAM-NANAC CHALK 1008 VCLCANICASST CHALK 0.87 VOLCANICASST CHALK 0.39 CLAYEY CHALK}

0:13 DOLOMIT. CALCARENITE

$$
\text { CL LITHCLCGY }
$$

1.32 FCKAM-AANNC OOZE

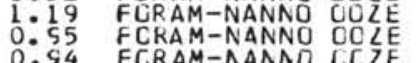
0.95 FCRAM-NANNC UUZE 0.88 FCKAM-NANAO LCLE $0: 88$ FORAM-NANNO UDZE 0. IC FORAM-AAMAC UCZE :
01
0 0.78 FCKAM-NANNC CCZE 0.34
0.54
0 FCRAM-NANNC UOZE 
ITE $100: L A T 24$ DEG 4 NIN N; LCNG 73 JEG $+811 \mathrm{~N}$ W; DEPTH $5325 \mathrm{M}$ (ANAL WALLACE)

$$
\begin{aligned}
& \text { SANPLE DEPTH SIL2 TIC2 ALZL3 FEZU3 MNO MGO CAO NA2O K2O P2O5 CL L L }
\end{aligned}
$$

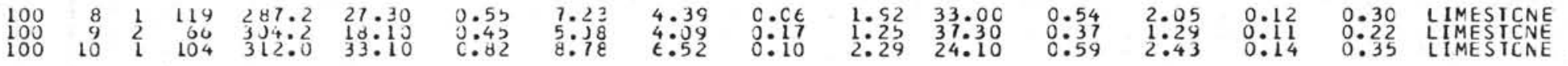

SITE 10L:LAT 2; DEG 12 NIN $N$;LCNG 74 JEG LS MIN W;DEPIH $48 C 8$ M (ANAL BUDD)

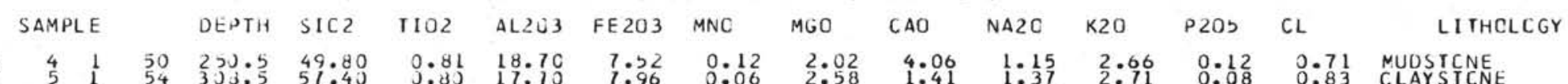

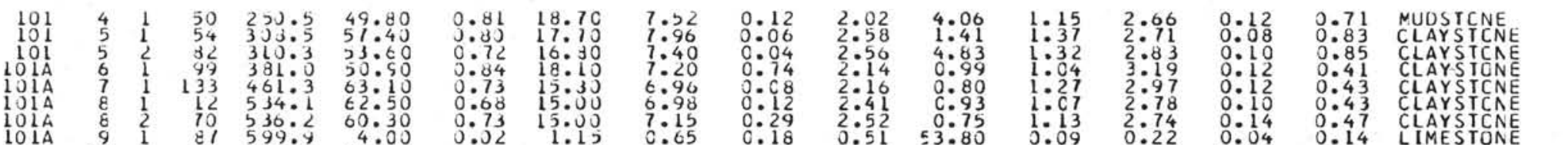

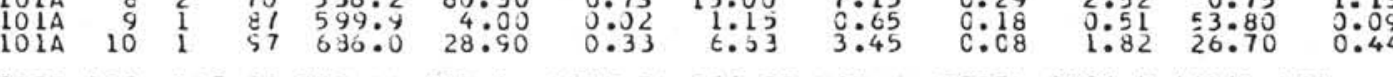

\begin{tabular}{|c|c|c|c|c|c|c|c|c|c|c|c|}
\hline SANPLE & & UEPTH & SIU2 & T $1 \cup 2$ & $A L 2 O 3$ & FE 203 & MNO & $M G O$ & CAO & $\mathrm{N} A 2 \mathrm{O}$ & K20 \\
\hline $\begin{array}{rr}1 & 1 \\
1 & 6 \\
2 & 3 \\
2 & 6 \\
3 & 2 \\
4 & 1 \\
4 & 4 \\
5 & 6 \\
6 & 6 \\
7 & 6 \\
8 & 1 \\
9 & 6 \\
10 & 6 \\
11 & 3 \\
12 & 4 \\
13 & 4 \\
14 & 2 \\
15 & 1 \\
16 & 1 \\
17 & 1 \\
18 & 1 \\
18 & 4 \\
19 & 1\end{array}$ & $\begin{array}{r}17 \\
80 \\
78 \\
80 \\
68 \\
124 \\
72 \\
78 \\
103 \\
77 \\
84 \\
70 \\
65 \\
73 \\
77 \\
75 \\
34 \\
77 \\
65 \\
70 \\
68 \\
80 \\
67\end{array}$ & 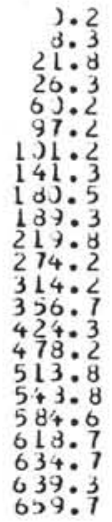 & $\begin{array}{l}37: 60 \\
46: 70 \\
53: 70 \\
48: 50 \\
49: 00 \\
36: 00 \\
49: 50 \\
37: 20 \\
41: 50 \\
50: 50 \\
45: 30 \\
42: 00 \\
48: 60 \\
47: 10 \\
47: 60 \\
49: 20 \\
45: 50 \\
40: 40 \\
50: 30 \\
50: 30 \\
48: 20 \\
48: 70 \\
47: 00\end{array}$ & $\begin{array}{l}0.60 \\
0: 73 \\
0: 78 \\
0: 64 \\
0: 74 \\
0: 56 \\
0: 84 \\
0: 64 \\
0: 61 \\
0: 82 \\
0: 73 \\
0: 75 \\
0: 74 \\
0: 71 \\
0: 68 \\
0: 72 \\
0: 66 \\
0: 66 \\
0: 70 \\
0: 68 \\
0: 70 \\
0: 77 \\
0: 70\end{array}$ & $\begin{array}{l}11.30 \\
14: 30 \\
15: 30 \\
13: 30 \\
14: 30 \\
11: 30 \\
17: 30 \\
14: 200 \\
12: 30 \\
17: 30 \\
15: 30 \\
15: 30 \\
15: 100 \\
15: 30 \\
15: 20 \\
14: 20 \\
13: 30 \\
14: 70 \\
14: 20 \\
14: 30 \\
17: 30 \\
17.10\end{array}$ & $\begin{array}{l}4.75 \\
6: 01 \\
6: 46 \\
5: 51 \\
5: 68 \\
5: 38 \\
7: 71 \\
5: 89 \\
5: 66 \\
6: 71 \\
6: 61 \\
6: 50 \\
6: 86 \\
6: 41 \\
6: 10 \\
6: 49 \\
5: 85 \\
5: 97 \\
6: 00 \\
6: 21 \\
6: 04 \\
6: 40 \\
5: 94\end{array}$ & $\begin{array}{l}c: c 9 \\
0: 15 \\
0: 16 \\
0: 17 \\
0: 14 \\
0: 16 \\
0: 11 \\
0: 21 \\
0: 36 \\
0: 17 \\
0: 13 \\
0: 17 \\
0: 16 \\
0: 20 \\
0: 20 \\
0: 23 \\
0: 28 \\
0: 27 \\
0: 24 \\
0: 24 \\
0: 22 \\
0: 26 \\
0: 39\end{array}$ & $\begin{array}{l}2: 36 \\
3: 50 \\
3: 00 \\
2: 59 \\
2: 41 \\
2: 26 \\
2: 89 \\
3: 15 \\
2: 46 \\
3: 23 \\
2: 66 \\
2: 85 \\
2: 53 \\
2: 42 \\
2: 22 \\
2: 44 \\
2: 21 \\
2: 20 \\
2: 44 \\
2: 39 \\
2: 16 \\
2: 25 \\
2: 12\end{array}$ & $\begin{array}{r}17.60 \\
8: 29 \\
3: 99 \\
8: 01 \\
7: 50 \\
17: 80 \\
3: 29 \\
9: 94 \\
13: 40 \\
3: 74 \\
7: 91 \\
9: 11 \\
5: 08 \\
8: 33 \\
7: 82 \\
6: 48 \\
10: 00 \\
11: 50 \\
6: 55 \\
6: 75 \\
8: 77 \\
3: 118 \\
7.09\end{array}$ & $\begin{array}{l}1: 50 \\
1: 59 \\
1: 86 \\
1: 97 \\
1: 62 \\
1: 24 \\
1: 45 \\
2: 11 \\
1: 41 \\
2: 30 \\
2: 23 \\
1: 19 \\
1003 \\
0: 94 \\
0: 37 \\
0: 88 \\
0: 58 \\
0: 55 \\
1: 04 \\
1: 44 \\
1: 00 \\
0: 02\end{array}$ & $\begin{array}{l}2.19 \\
3 \\
3: 08 \\
3: 35 \\
2: 83 \\
2: 75 \\
2: 35 \\
3: 62 \\
2: 93 \\
2: 43 \\
3: 35 \\
2: 99 \\
3: 03 \\
2: 74 \\
2: 58 \\
2: 55 \\
2: 52 \\
2: 45 \\
2: 39 \\
2: 37 \\
2: 41 \\
2: 46 \\
2: 48 \\
2: 29\end{array}$ \\
\hline
\end{tabular}

SITE 102: LAT 30 DE 44 NIN N; LCNG 74 CEG 27 NIN W; CEPTH 3426 M (ANAL LI)

SITE 104: LAT 30 DEG 50 MIN N; LCAG 74 CEG 20 NIN W; DEPIH 3811 M (ANAL LI) DEPIH SIU2 TIO2 AL?O3 FE2U3 MNO

MGC

CAO NA2C

K20

$05 \mathrm{CL}$ $\mathrm{L}$

LITHCLCGY

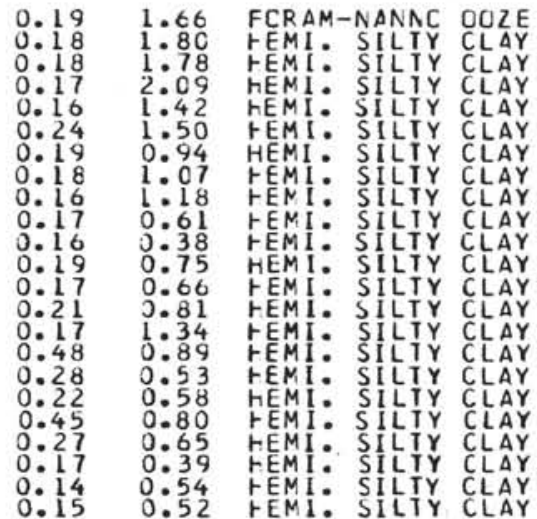

$0.14 \quad 0.54$ FEMI: SILYY CLAY

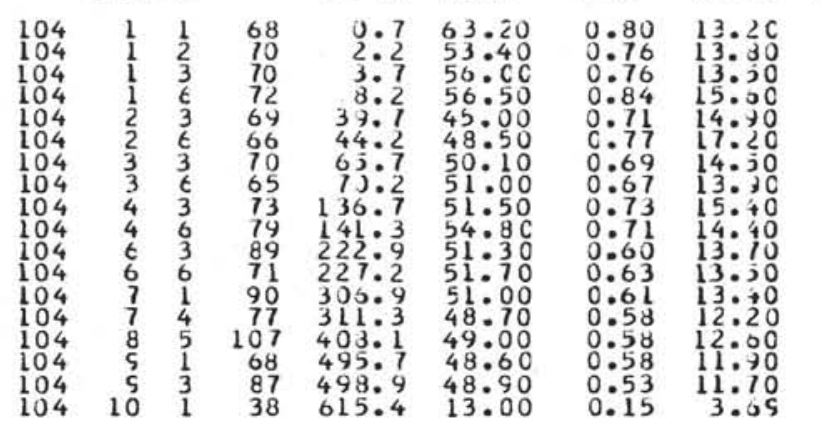

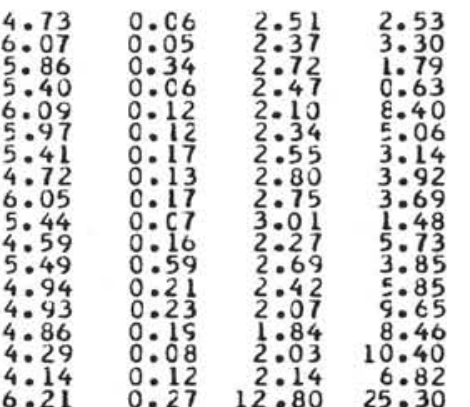

$1: 47$
$1: 21$
$1: 40$
$0: 87$
$0: 56$
$0: 65$
$1: 13$
$1: 13$
$0: 82$
$1: 02$
$0: 67$
$0: 71$
$0: 69$
$0: 93$
$1: 12$
$0: 92$
$0: 80$
0.21

P205 CL

LITHOLCGY

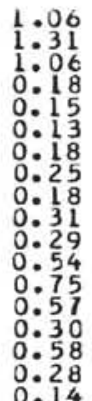

$1 \cdot 9$

1.92 HEM1. SILTY CLAY

$1: 76$ HEMI: SILY CLAY

$1: 49$ HEMI: SILIY CLAY

2.50 HEMI: SILTY CLAY

1012 HEMI: SILTY CLAY

:05 HEMI: SILTY CLAY

0.81 HEMI: SILIY CLAY

0.57 HEMI: SILIY CLAY

1.11 HEMI: SILIY CLAY 
SITE 105:LAT 34 DEG 54 NIN N ;LCNG 69 DEG $1 J$ MIA W:UEPTH 5261 M (ANAL BUDO)

P205

$\mathrm{CL}$

LITHOLOGY

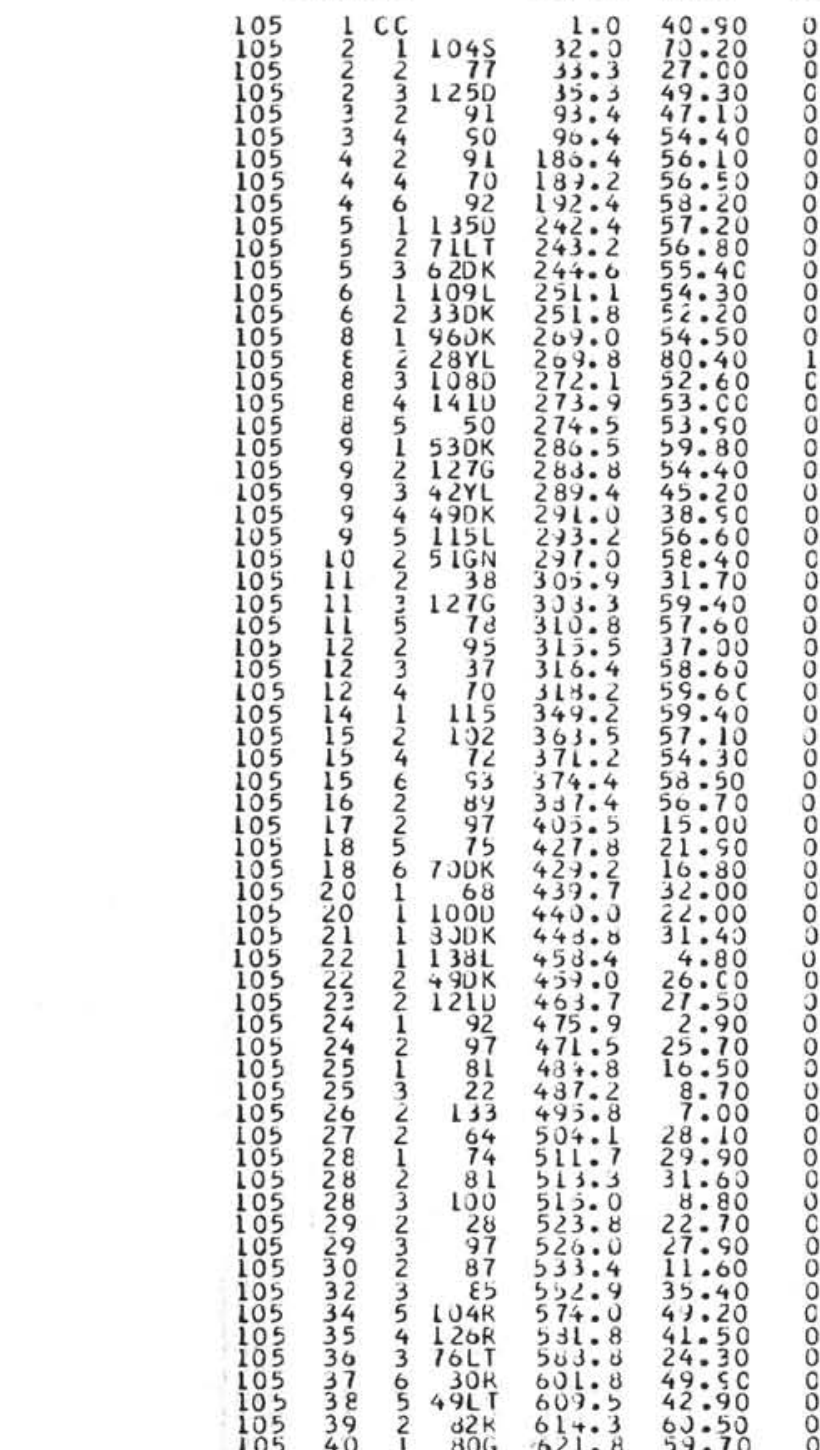

\begin{tabular}{|c|c|c|c|c|c|}
\hline 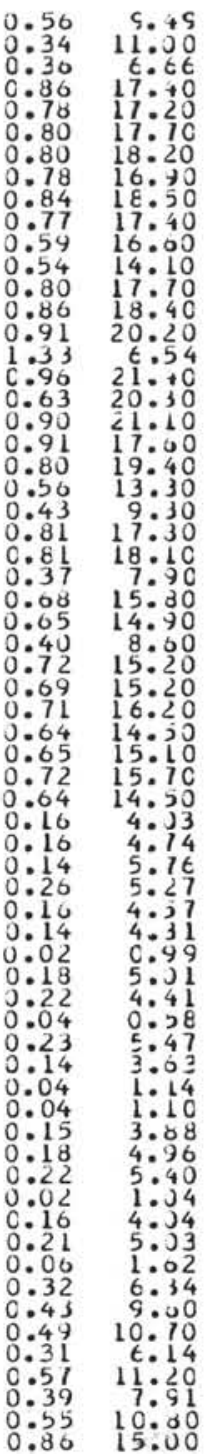 & $\begin{array}{l}4: 54 \\
2: 68 \\
3: 64 \\
7: 80 \\
6: 88 \\
7: 81 \\
7: 44 \\
7: 94 \\
6: 00 \\
6: 41 \\
6: 06 \\
4: 50 \\
6: 38 \\
6: 77 \\
6: 70 \\
3: 13 \\
6: 61 \\
4: 22 \\
7: 26 \\
6: 71 \\
7: 81 \\
23: 20 \\
6: 49 \\
7: 42 \\
6: 69 \\
2: 38 \\
6: 04 \\
5: 80 \\
3: 59 \\
5: 92 \\
5: 58 \\
6: 20 \\
5: 65 \\
5: 96 \\
5: 87 \\
5: 57 \\
1: 56 \\
1: 85 \\
2: 28 \\
2: 48 \\
2: 93 \\
1: 82 \\
6: 50 \\
2: 25 \\
2: 35 \\
0: 39 \\
2: 05 \\
1: 85 \\
0: 43 \\
0: 50 \\
1: 50 \\
2: 00 \\
2: 04 \\
0: 55 \\
1: 41 \\
2: 07 \\
0: 61 \\
3: 58 \\
5: 45 \\
7: 07 \\
2: 43 \\
6: 50 \\
2: 88 \\
6: 05 \\
6: 46\end{array}$ & 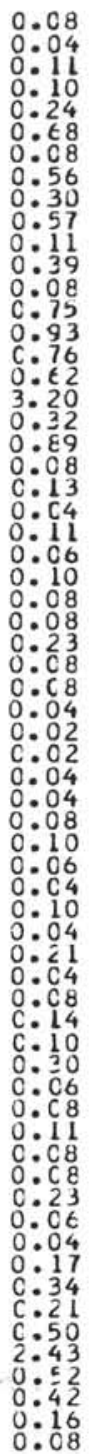 & 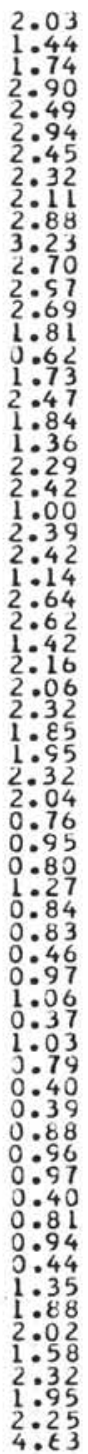 & $\begin{array}{r}16: 90 \\
5: 24 \\
27: 10 \\
2: 89 \\
3: 72 \\
c: 72 \\
0: 29 \\
0: 46 \\
0: 49 \\
0: 63 \\
0: 66 \\
1: 47 \\
0: 46 \\
0: 65 \\
0: 49 \\
0: 24 \\
0: 28 \\
0: 42 \\
0: 38 \\
0: 63 \\
0: 48 \\
0: 39 \\
0: 48 \\
0: 49 \\
0: 30 \\
22: 40 \\
0: 48 \\
2: 17 \\
22: 60 \\
1: 07 \\
0: 81 \\
0: 48 \\
0: 58 \\
0: 44 \\
0: 73 \\
0: 72 \\
35: 60 \\
34: 30 \\
35: 90 \\
25: 30 \\
31: 80 \\
28: 30 \\
55: 00 \\
28: 40 \\
28: 40 \\
55: 10 \\
33: 70 \\
40: 60 \\
54: 10 \\
52: 70 \\
35: 70 \\
22: 50 \\
32: 80 \\
45: 80 \\
38: 60 \\
33: 80 \\
48: 70 \\
27: 20 \\
15: 20 \\
16: 50 \\
31: 50 \\
10: 50 \\
21: 50 \\
6: 64 \\
1: 19\end{array}$ & $\begin{array}{l}1: 21 \\
3: 12 \\
1: 18 \\
1: 30 \\
1: 23 \\
1: 24 \\
: 22 \\
1: 24 \\
1: 04 \\
1: 47 \\
1: 67 \\
1: 03 \\
0: 88 \\
0: 81 \\
0: 41 \\
1: 29 \\
0: 91 \\
0: 86 \\
1: 03 \\
0: 89 \\
1: 51 \\
1: 20 \\
1: 29 \\
1: 05 \\
1: 65 \\
1: 72 \\
1: 23 \\
1: 77 \\
1: 61 \\
1: 72 \\
1: 88 \\
1: 72 \\
1: 78 \\
0: 47 \\
0: 48 \\
0: 36 \\
0: 52 \\
0: 45 \\
0: 54 \\
0: 03 \\
0: 60 \\
0: 55 \\
0: 00 \\
0: 49 \\
0: 35 \\
0: 10 \\
0: 09 \\
0: 37 \\
0: 42 \\
0: 44 \\
0: 10 \\
0: 30 \\
0: 35 \\
0: 14 \\
0: 54 \\
0: 80 \\
0: 75 \\
0: 43 \\
0: 77 \\
0: 54 \\
0: 52 \\
1: 44\end{array}$ \\
\hline
\end{tabular}

SITE 106: LAT 36 UE $\angle 6$ NIN N; LLAG 69 CEG 23 NIN W: OEPIH 4500 M (ANAL WALLACEI

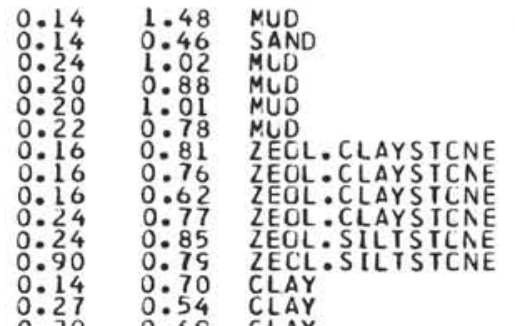

0.69 CLAY 0.37 YELLOK CLAY

0.66 CLAY

0.72 CLAY

0.54 GREEA ZECL.CLAYSTONE

.

0.32 ZEULLIIC CLAYSICNE

0.54 GREEA ZECL.CLAYSTUNE

O:54 GREEN LECL.CLAYSTONE

0.56 GREEN ZEOL:CLAYSTONE

0.62 GREEN LECL:CLAYSTONE
0.66 GREEN ZECL:CLAYSTONE

$0: 72$ GREEN LECL:CLAYSTCNE

$0: 54$ GREEN ZECL:CLAYSTUNE

O.61 GREEN 2EOL.CLAYSTSN.

0.64 BLACK LECL:DLAYST.
0.75 ZECLITIC CIAYSTCNE

0.55 LINESYCNE LY LIMESTCNE

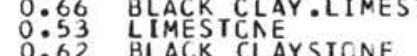

0.62
0.62
0.67 CACK CLAYSICNE

$0.6 C$ LIMESTCNE TICNE

0.55 CARK LINESTCNE

0.53 LIMESICNE

0.50 LIMESTCNE

0.28 LIMESTCAE

0.27 LIMESTCNE

0.39 LIMESTCNE

0.43 CLAYEY LIMESTCNE

0.33 LIMESICNE

0.53

0.31 LIMESTCAE

0.23 FERRUG. LIMESTCNE

0.3

O

0.26 THIN GRAY LIMESTCNE

$0: 42$ FERRUG LLIMESTCNE
0.20 GREEA CLAY FRAGENT

SAMHLE DEPIH SIO2 TIO2 AL2U3 FE2O3 MNO MGO CAO NA2C

106

3.9 56. 20

$0.87 \quad 16.10$

$\begin{array}{lllll}0.06 & 2.60 & 2.97 & 1.45 & 3.45\end{array}$

205

$\mathrm{CL}$

LITHCLCGY

1.41 TEMIPELAGIC MUD 


\begin{tabular}{|c|c|c|c|c|c|c|c|c|c|c|c|c|c|c|c|c|}
\hline \multicolumn{4}{|c|}{ SANPLE } & \multirow[b]{2}{*}{$\begin{array}{r}\text { UESTH } \\
\text { J.7 } \\
40: 3 \\
112: 1 \\
341: 9 \\
309: 7 \\
452: 9 \\
535: 5 \\
559: 9 \\
737: 0 \\
937: 8 \\
942: 8 \\
955: 9\end{array}$} & \multirow[b]{2}{*}{$\begin{array}{l}\text { s1c2 } \\
53.50 \\
58: 40 \\
57: 10 \\
51: 70 \\
54: 50 \\
54: 50 \\
46: 50 \\
56: 50 \\
58: 00 \\
45: 00 \\
59: 80 \\
62.40\end{array}$} & \multirow[b]{2}{*}{$\begin{array}{l}\text { IIC2 } \\
0.80 \\
0: 04 \\
0.61 \\
0: 71 \\
0: 70 \\
0.78 \\
0: 66 \\
0: 76 \\
0.79 \\
0.67 \\
0.63 \\
0.68\end{array}$} & \multirow[b]{2}{*}{$\begin{array}{l}\text { AL } 2 C 3 \\
16.30 \\
13: 80 \\
11: 2 C \\
13: 50 \\
17: 00 \\
18: 10 \\
14: 30 \\
17: 40 \\
17.50 \\
13: 50 \\
14: 10 \\
14.90\end{array}$} & \multirow[b]{2}{*}{$\begin{aligned} \text { FE } 203 \\
7.25 \\
5: 53 \\
4: 69 \\
5: 52 \\
5: 52 \\
6: 59 \\
5: 93 \\
6: 41 \\
6: 11 \\
5: 13 \\
5: 32 \\
5.37\end{aligned}$} & \multirow[b]{2}{*}{$\begin{array}{l}\text { NAC } \\
0.05 \\
0: 04 \\
C: C 6 \\
0: 04 \\
0: 17 \\
0: 16 \\
0: 31 \\
0: C 8 \\
0: 17 \\
0: 15 \\
0: C 7 \\
0 . C 3\end{array}$} & \multirow[b]{2}{*}{$\begin{array}{l}M G O \\
2.82 \\
2: 98 \\
2: 04 \\
2.60 \\
2: 51 \\
2: 55 \\
2: 34 \\
2.54 \\
2: 42 \\
2: 89 \\
2.20 \\
2.28\end{array}$} & \multirow[b]{2}{*}{$\begin{array}{r}C A O \\
3.04 \\
5: 59 \\
5: 80 \\
7: 43 \\
3: 99 \\
2: 02 \\
10: 70 \\
2: 01 \\
0: 73 \\
12: 70 \\
3: 10 \\
0.54\end{array}$} & \multirow[b]{2}{*}{$\begin{array}{l}\text { NA2C } \\
1: 73 \\
2: 33 \\
1: 54 \\
1: 43 \\
1: 23 \\
1: 41 \\
1: 21 \\
1: 32 \\
1: 11 \\
1: 95 \\
1: 15 \\
1: 30\end{array}$} & \multirow[b]{2}{*}{$\begin{array}{l}k 20 \\
3.55 \\
2: 73 \\
2: 54 \\
2: 69 \\
2: 73 \\
2: 90 \\
2: 53 \\
2: 87 \\
2.68 \\
3: 11 \\
2=10 \\
2.00\end{array}$} & \multirow[b]{2}{*}{$\begin{array}{l}\text { P205 } \\
0.18 \\
0: 17 \\
0: 14 \\
0: 15 \\
0: 16 \\
0: 17 \\
0: 23 \\
0: 17 \\
0: 14 \\
0: 25 \\
0: 21 \\
0.15\end{array}$} & \multirow[b]{2}{*}{$\begin{array}{l}\text { CL } \\
0.72 \\
0: 80 \\
0: 92 \\
0: 81 \\
0: 47 \\
0: 49 \\
0: 53 \\
0: 51 \\
0: 50 \\
0: 8 C \\
0: 47 \\
0.31\end{array}$} & \multirow[b]{2}{*}{ 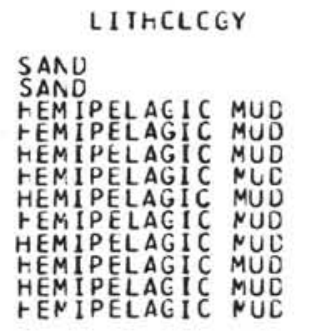 } \\
\hline $\begin{array}{l}6 \\
6 \\
6 \\
6 \\
68 \\
68 \\
68 \\
68 \\
6 \mathrm{~B}\end{array}$ & $\begin{array}{l}1 \\
2 \\
3 \\
6 \\
1 \\
1 \\
2 \\
3 \\
3 \\
4 \\
5 \\
5 \\
6\end{array}$ & $\begin{array}{l}5 \\
1 \\
2 \\
2 \\
3 \\
3 \\
2 \\
2 \\
5 \\
3 \\
3 \\
2 \\
6 \\
3\end{array}$ & $\begin{array}{r}65 \\
126 \\
120 \\
36 \\
73 \\
44 \\
45 \\
92 \\
64 \\
132 \\
27 \\
92\end{array}$ & & & & & & & & & & & & & \\
\hline \multirow[t]{2}{*}{ TE } & & & T 34 & 1 & NIA $N$; & LCNG 46 & & & DEPIt & $3657 \mathrm{MI}$ & I $\triangle \wedge A L L I$ & & & & & \\
\hline & AMPL & & & DEPTH & SIC2 & 1102 & $A L \geq 03$ & FE 203 & MNC & MGO & CAO & $\mathrm{NA} 2 \mathrm{C}$ & K20 & P205 & $C L$ & LITHCLCGY \\
\hline & $\begin{array}{l}1 \\
1 \\
1 \\
3 \\
2 \\
3 \\
2 \\
4 \\
4 \\
5 \\
3 \\
4 \\
5 \\
5 \\
7 \\
5 \\
11 \\
12 \\
13 \\
14 \\
15 \\
16\end{array}$ & $\begin{array}{l}6 \\
1 \\
5 \\
2 \\
2 \\
3 \\
6 \\
6 \\
3 \\
5 \\
2 \\
6 \\
6 \\
2 \\
3 \\
6 \\
2 \\
2 \\
4 \\
1 \\
5 \\
5 \\
6 \\
1\end{array}$ & $\begin{array}{r}36 \\
140 \\
69 \\
87 \\
57 \\
68 \\
68 \\
61 \\
66 \\
93 \\
68 \\
90 \\
65 \\
80 \\
46 \\
66 \\
87 \\
88 \\
84 \\
82 \\
54 \\
60\end{array}$ & $\begin{array}{r}35: 9 \\
33: 4 \\
87: 8 \\
99: 4 \\
133: 6 \\
105: 2 \\
100: 2 \\
107: 6 \\
1112: 7 \\
117: 4 \\
158: 2 \\
202: 4 \\
273: 0 \\
27803 \\
290: 0 \\
30302 \\
329: 4 \\
384: 9 \\
447: 8 \\
50508 \\
586: 4 \\
652: 0\end{array}$ & $\begin{array}{l}36.80 \\
58: 80 \\
45: 4 c \\
58: 60 \\
56: 60 \\
58: 20 \\
63: 00 \\
63: 40 \\
61: 70 \\
32: 00 \\
44: 30 \\
51: 20 \\
37: 40 \\
57: 10 \\
48: 20 \\
58: 60 \\
42: 30 \\
46: 50 \\
49: 10 \\
47: 80 \\
47: 70 \\
63.80\end{array}$ & $\begin{array}{l}0.52 \\
0: 50 \\
0: 72 \\
0: 81 \\
0: 75 \\
0: 89 \\
0: 72 \\
0: 72 \\
0: 79 \\
0: 53 \\
0: 61 \\
0: 73 \\
0: 59 \\
0: 65 \\
0: 69 \\
0: 84 \\
0: 39 \\
0: 50 \\
0: 54 \\
0: 50 \\
0: 53 \\
0: 56\end{array}$ & $\begin{array}{r}5.05 \\
14: 50 \\
12: 70 \\
13: 60 \\
13: 40 \\
13: 50 \\
14: 90 \\
15: 40 \\
14: 60 \\
7: 08 \\
10: 30 \\
13: 50 \\
8: 90 \\
10: 20 \\
11: 20 \\
13: 10 \\
7: 50 \\
9: 38 \\
12: 10 \\
11: 50 \\
11: 50 \\
13.30\end{array}$ & $\begin{array}{l}3.76 \\
5: 05 \\
5: 38 \\
6: 06 \\
5: 08 \\
6: 67 \\
5: 09 \\
6: 55 \\
6: 23 \\
5: 60 \\
4: 39 \\
6: 25 \\
4: 06 \\
5: 25 \\
4: 69 \\
5: 70 \\
3: 05 \\
4: 61 \\
5: 25 \\
5: 16 \\
5: 07 \\
7: 74\end{array}$ & $\begin{array}{l}0: 12 \\
0: 09 \\
c: 17 \\
0: 26 \\
0: 14 \\
0: 16 \\
0: 07 \\
0: 05 \\
0: c 6 \\
0: 12 \\
0: 06 \\
0: 11 \\
0: 09 \\
0: 06 \\
0: 10 \\
0: 13 \\
0: 12 \\
0: 10 \\
0: t 4 \\
0: 06 \\
0: 17 \\
0: 05\end{array}$ & $\begin{array}{l}2.22 \\
1: 34 \\
1: 82 \\
1: 75 \\
1: 83 \\
1: 98\end{array}$ & $\begin{array}{r}18.9 \\
5: 4 \\
13: 10 \\
5: 2 \\
7: 4 \\
5: 2 \\
2: 4 \\
1: 7 \\
2: 7 \\
22: 7 \\
16: 7 \\
8: 3 \\
15: 6 \\
11: 3 \\
11.4 \\
9: 9 \\
14: 2 \\
12: 6 \\
13: 4 \\
14: 0 \\
1: 2\end{array}$ & $\begin{array}{l}1: \\
1: \\
1: \\
1: \\
1: \\
1: \\
1: \\
1: \\
1: \\
1: \\
1: \\
1: \\
0:\end{array}$ & $\begin{array}{l}1 \\
2 \\
2 \\
2 \\
2 \\
2 \\
2 \\
2\end{array}$ & 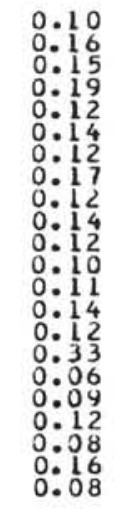 & & 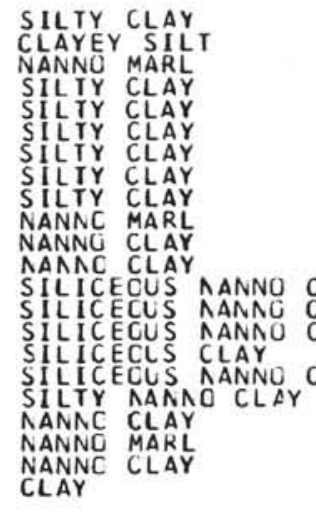 \\
\hline \multirow[t]{2}{*}{ I IE } & $6:$ & LAT & I 57 & UEG & MIN & UNG 15 & C CEG & $M I N$ & & $1151 \mathrm{M}$ & AL & & & & & \\
\hline & MPL & & & EPTH & & 02 & $A L 2$ & FE2 & MNO & MGC & & A & & & & CGY \\
\hline & 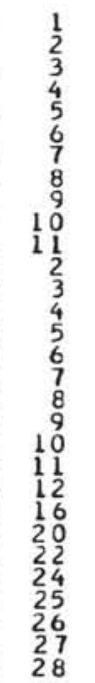 & $\begin{array}{l}1 \\
3 \\
6 \\
3 \\
2 \\
2 \\
4 \\
6 \\
3 \\
5 \\
6 \\
6 \\
2 \\
6 \\
6 \\
5 \\
4 \\
3 \\
3 \\
33 \\
3 \\
6 \\
6 \\
2 \\
5 \\
6 \\
1 \\
5 \\
4 \\
3 \\
1\end{array}$ & $\begin{array}{r}74 \\
130 \\
130 \\
90 \\
1110 \\
112 \\
93 \\
84 \\
84 \\
120 \\
55 \\
68 \\
78 \\
80 \\
75 \\
87 \\
75 \\
92 \\
85 \\
76 \\
75 \\
80 \\
76 \\
76 \\
130 \\
138 \\
73 \\
78 \\
94 \\
131\end{array}$ & $\begin{array}{r}0.7 \\
13: 3 \\
26: 5 \\
33: 9 \\
38: 7 \\
50: 6 \\
62: 4 \\
66: 8 \\
73: 8 \\
89: 7 \\
104: 1 \\
1111: 2 \\
167: 3 \\
217: 3 \\
265: 8 \\
314: 4 \\
362: 8 \\
412: 9 \\
462: 8 \\
512: 8 \\
567: 3 \\
607: 3 \\
673: 7 \\
70778 \\
727: 8 \\
760: 4 \\
812: 7 \\
830: 3 \\
834: 9 \\
841.3\end{array}$ & $\begin{array}{r}12: 70 \\
19: 80 \\
16: 60 \\
19: 30 \\
12: 60 \\
16: 20 \\
10: 80 \\
3: 81 \\
6: 22 \\
2: 20 \\
2: 20 \\
2: 00 \\
3: 00 \\
3: 40 \\
5: 20 \\
2: 00 \\
1: 80 \\
3: 60 \\
3: 60 \\
6: 80 \\
3: 60 \\
12: 20 \\
13.40 \\
7: 20 \\
8.60 \\
8.60 \\
8: 00 \\
10.80 \\
1.80\end{array}$ & $\begin{array}{l}0: 1 \\
0: 0 \\
1: 3 \\
0: 2 \\
0: 3 \\
0: 1 \\
0: 3 \\
1: 2 \\
0: 0 \\
0: 1 \\
0: 0 \\
0: 0 \\
0: 0 \\
0: 0 \\
0: 0 \\
0: 0 \\
0: 0 \\
0: 0 \\
0: 0 \\
0: 0 \\
0: 0 \\
0: 0 \\
0: 4 \\
0: 0 \\
0: 0\end{array}$ & $\begin{array}{l}0: \\
0 \\
0 \\
1: \\
0 \\
0: \\
0 \\
0: \\
0: \\
c \\
3: \\
0\end{array}$ & $\begin{array}{l}1 \\
1 \\
0 \\
0 \\
0 \\
0 \\
0 \\
0 \\
0 \\
0 \\
0\end{array}$ & $\begin{array}{l}0 . \\
0: \\
0: \\
0: \\
0: \\
0 \\
0: \\
0: \\
0: \\
0: \\
0: \\
0: \\
0: \\
0: \\
0 \\
0: \\
0:\end{array}$ & $\begin{array}{l}0: \\
1: \\
0 \\
0: \\
0: \\
0: \\
0: \\
0: \\
0: \\
0: \\
0: \\
0: \\
0: \\
0: \\
0: \\
0: \\
0: \\
0:\end{array}$ & $\begin{array}{l}44 \\
21 \\
33 \\
36 \\
35 \\
40 \\
39 \\
40 \\
48 \\
49 \\
51 \\
50 \\
51 \\
52 \\
49 \\
50 \\
51 \\
50 \\
51 \\
48 \\
45 \\
50 \\
34 \\
42 \\
46 \\
46 \\
48 \\
45\end{array}$ & & & & & 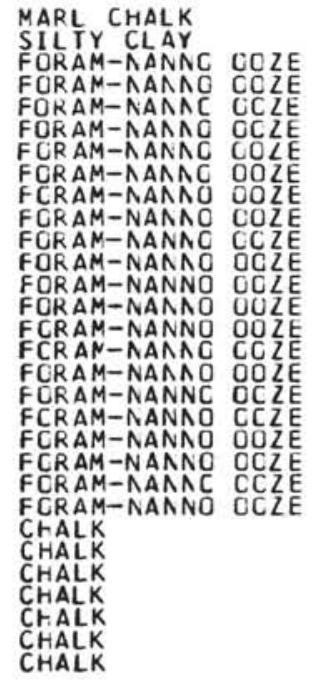 \\
\hline
\end{tabular}


SITE 118: LAT 45 DEG 3 MIN NiLONG 9 DEG $1 N$ IN $n$; DEPTH 4SO 1 M (ANAL BUDD)
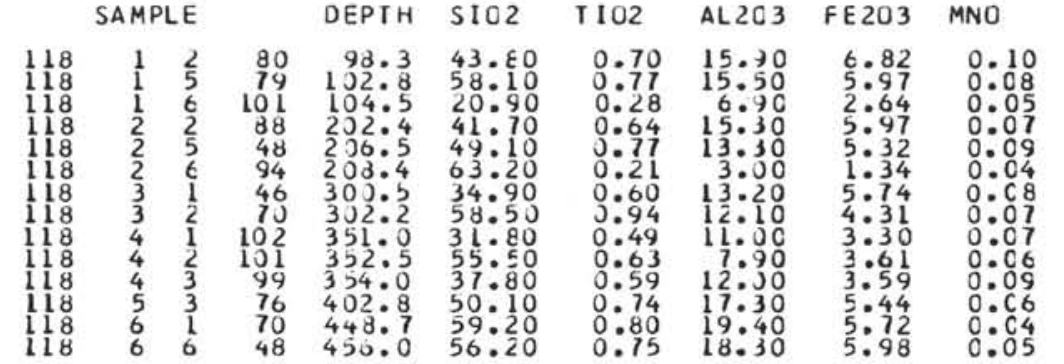

MGO

CAO NA2C

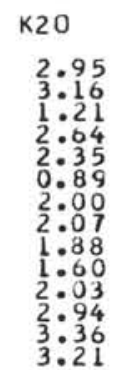

P205

$\mathrm{CL}$

LITHCLCGY

SITE LI9: LAT 45 DEG 2 NIN $A$; LLNG 7 LEG 58 MIN W; DEPTH $4447 \mathrm{M}$ (ANAL. LI)
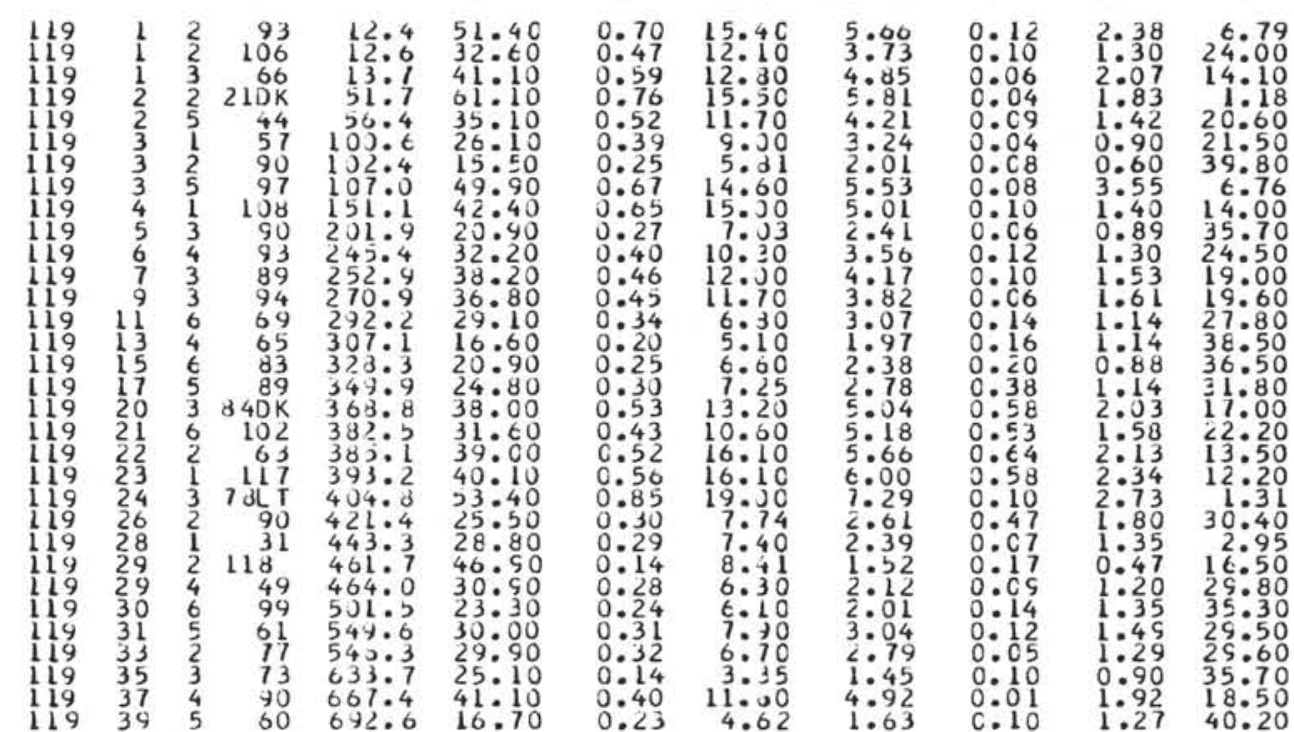

NA20

K20

0.15
$0: 15$
$0: 08$
$0: 10$
$0: 11$
$0: 12$
$0: 16$
$0: 11$
$0: 08$
$0: 11$
$0: 08$
0.12

0.88 SILIY CLAY

$1: 32$ MARLY CLAY

0.66 CLAY

Siltr clay

0.73 SILTY CLAY

0.76 CLAY CLAY

0.65 CLAY

$\begin{array}{lll}0: 14 & 0: 78 & \text { CLAY } \\ 0.17 & 0.78 & \text { CLAY }\end{array}$

SITE 140: LAT 21 DEG 45 MIN N: LCAG 21 CEG 43 NIN W: CEPTH 4483 N (ANAL LI)

\begin{tabular}{|c|c|c|c|c|c|c|c|c|c|c|}
\hline $\begin{array}{l}1 \\
1 \\
1 \\
1 \\
2 \\
2 \\
2 \\
2 \\
2 \\
2 \\
3 \\
3 \\
4 \\
4 \\
6 \\
7\end{array}$ & $\begin{array}{l}2 \\
4 \\
6 \\
1 \\
1 \\
3 \\
5 \\
3 \\
6 \\
1 \\
3 \\
1 \\
3 \\
2 \\
1\end{array}$ & $\begin{array}{r}42 \\
129 \\
47 \\
128 \\
40 \\
70 \\
71 \\
138 \\
106 \\
85 \\
26 \\
22 \\
128 \\
80 \\
145\end{array}$ & $\begin{array}{r}90: 9 \\
94: 8 \\
97: 0 \\
151: 3 \\
201: 5 \\
204: 8 \\
237: 7 \\
233: 1 \\
243: 6 \\
311: 9 \\
314: 3 \\
363: 2 \\
372: 3 \\
512: 4 \\
586: 4\end{array}$ & $\begin{array}{r}4.40 \\
3: 60 \\
8: 00 \\
52: 40 \\
63: 00 \\
64: 60 \\
62: 10 \\
57: 90 \\
56: 20 \\
53: 80 \\
33: 50 \\
56: 20 \\
5490 \\
57.60 \\
75.60\end{array}$ & $\begin{array}{l}0.08 \\
0: 06 \\
0: 29 \\
0: 94 \\
0: 45 \\
0: 50 \\
0: 42 \\
0: 87 \\
0: 84 \\
0: 16 \\
0: 78 \\
0: 06 \\
0: 60 \\
0: 31 \\
0.40\end{array}$ & $\begin{array}{r}1: 30 \\
1: 40 \\
20: 34 \\
20: 30 \\
8: 30 \\
9: 30 \\
8: 30 \\
17020 \\
5: 70 \\
5: 30 \\
15: 00 \\
13: 100 \\
13: 00 \\
6: 40 \\
9: 00\end{array}$ & $\begin{array}{l}0.80 \\
0: 58 \\
1: 40 \\
7.84 \\
3: 36 \\
3: 84 \\
3: 97 \\
5: 35 \\
5: 97 \\
5: 09 \\
5: 55 \\
5: 08 \\
4.85 \\
2.73 \\
3.27\end{array}$ & $\begin{array}{l}0: 05 \\
0: 04 \\
c: 05 \\
0: 03 \\
0: 04 \\
0: 03 \\
0: 04 \\
c: 02 \\
c: 04 \\
0: 04 \\
0: 04 \\
0: 04 \\
0: 03 \\
0: 02 \\
0: 02\end{array}$ & $\begin{array}{l}0: 43 \\
0: 28 \\
0: 60 \\
2: 18 \\
1: 15 \\
1: 26 \\
1: 11 \\
2: 05 \\
3: 55 \\
3: 94 \\
4: 32 \\
5: 75 \\
4: 88 \\
7: 52 \\
2.37\end{array}$ & $\begin{array}{r}48.10 \\
49: 40 \\
45: 80 \\
0: 49 \\
5: 32 \\
3: 54 \\
4.37 \\
0.47 \\
0: 54 \\
2: 31 \\
0: 63 \\
1: 74 \\
3.17\end{array}$ \\
\hline
\end{tabular}

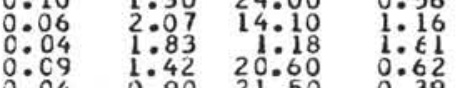

$\begin{array}{llll}0.08 & 0.60 & 39.80 & 0.33 \\ 0.10 & 1.45 & 6.76 & 1: 08 \\ 0 & 1.40 & 14.00 & 0.73\end{array}$

$0: 12 \quad 1.30$

$0.16 \quad 1.14 \quad 38.50$

$0 . \begin{array}{lll}38 & 1.14 & 31 \\ 5 & 2.03 & 17: 00\end{array}$

$0.64 \quad 2: 13 \quad 13: 50$

$\begin{array}{lll}0.10 & 2.73 & 1: 31 \\ 0: 47 & 1: 80 & 30: 40 \\ 0 & 1: 35 & 2.95\end{array}$

$0.17 \quad 0.47$

$\begin{array}{lll}1.45 & 29.50 & 0.89 \\ 1.29 & 25.60 & 0.61\end{array}$

0.89

0.37

20

K20

0.32

0.28

0.93

$1: 85$

1.46
1.46
1.54
1.52

$1: 54$
$0: 52$
1.03
P205 CL

$\begin{array}{lll}0.16 & 1.24 & \text { FCRAM-NANNO MUD } \\ 0.15 & 0: 79 & \text { FORAM-NANA MLU }\end{array}$

$\begin{array}{lll}0.14 & 1.55 & \text { FORAM-NANNC } \\ 0.16 & 0.79 & \text { SILIY CLAY }\end{array}$

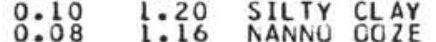

0.08 $0: 95$ NANNO CCZE

0.65 NANNO OOZE

0.77 NANNG CLAY

0.85
0.65 NANNO CLAY

0.66 CLAY

0.78 AANNO CLAY

0.52 NANNO CLAY

0.51 NANNO CLAY

0.64 CLAY

0.50 CLAY

00671 CLAY

0.54 CLAY

0:74 SAND

0.32 CLAYSTCNE

0.24 CLAYSTCNE

0.22 CLAYSTONE

$0.10 \quad 0.21$ CLAYSTINE

P205 CL

LITHOLOGY

$\begin{array}{lll}0.09 & 0.76 & \text { NANNO CHALK COZE } \\ 0.08 & 1.40 & \text { NANNO CHALK CCLE } \\ 0.11 & 1.45 & \text { NANNO CHALK CCZE }\end{array}$

$\begin{array}{lll}0.20 & 0.38 & \text { CLAY CHA } \\ 0.18 & 2: 00 & \text { SILICECLS MLD/COZE }\end{array}$

$\begin{array}{lll}0: 17 & 1: 78 & \text { SILICECLS MLCICOZE } \\ 0.11 & 2: 52 & \text { SILICECLS MLCIOOLE }\end{array}$

0.75 CLAY

$1: 26$
$1: 36$ CLAY

$1: 47$
0.17

$1: 83$
0.86
0 SHALE SILTY CLAY 

CNG 24 LEG C MIN $w$; DEPTH 4148 M (ANAL TERRANA)

DEPTH

$T 102$

AL LJJ3 FE2 203 NNC MGO CAO

NA2C K2O P2O5 CL

LITHOLCGY

CLAY/SILI/SANC

SAMPLE

$\begin{array}{ll}0.70 & 8.30 \\ 3: 35 & 5: 36 \\ 0: 08 & 1: 35 \\ 0: 38 & 5: 59 \\ 0.45 & 4: 73 \\ 0: 25 & 3: 72 \\ 0: 15 & 3: 30 \\ 0: 55 & 8: 34 \\ 0: 20 & 4: 16 \\ 0: 38 & 6: 44 \\ 0: 32 & 3: 67 \\ 0.35 & 2.05 \\ 0.92 & 17.00 \\ 1.21 & 20.10\end{array}$

$\begin{array}{ll}3.77 & 0.07 \\ 2: 15 & 0: 07 \\ c: 78 & 0: C 7 \\ 2: 76 & 0: 04 \\ 1: 97 & 0.06 \\ 1: 49 & 0: 05 \\ 1: 13 & 0: 06 \\ 3: 28 & 0: 06 \\ 1: 04 & 0: 07 \\ 2.65 & C: C 6 \\ 1: 84 & 0: 11 \\ 1: 70 & 0: 10 \\ 6: 38 & 0.31\end{array}$

$M G O$

$\begin{array}{ll}1.53 & 28.10 \\ 0.89 & 34.80 \\ 0.42 & 45.00 \\ 0: 86 & 32.00 \\ 0.86 & 34.60 \\ 0.89 & 35.50 \\ 0.60 & 41: 70 \\ 0.95 & 31.60 \\ 0.63 & 42.70 \\ 1: 01 & 32.60 \\ 0.76 & 40.50 \\ 0.63 & 44.550 \\ 2.32 & 8.32\end{array}$

$N A 2 C$
0.70
$0: 15$
$0: 23$
$0: 40$
$0: 50$
$0: 43$
$0: 31$
$0: 28$
$0: 04$
$0: 88$
$0: 27$
$0: 30$
$0: 78$
0.87

K20

$205 \mathrm{CL}$

LI THCLCGY
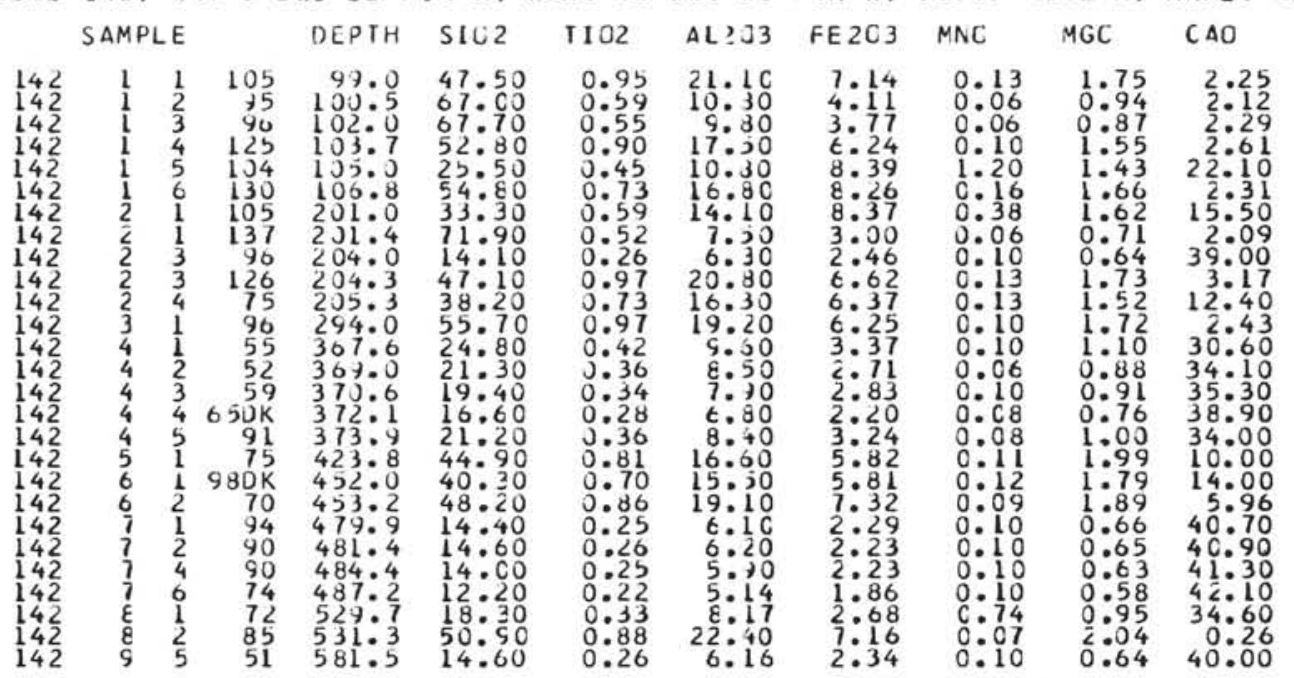

NA2O

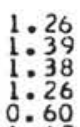

$1: 26$
$1: 39$
$1: 38$
0.26

.60
0.87

1.03
0.30
1.05

1.05
0.88
1.15

1. 15

0.43

0.49

0.83

0.20

SITE $14 t / 145$
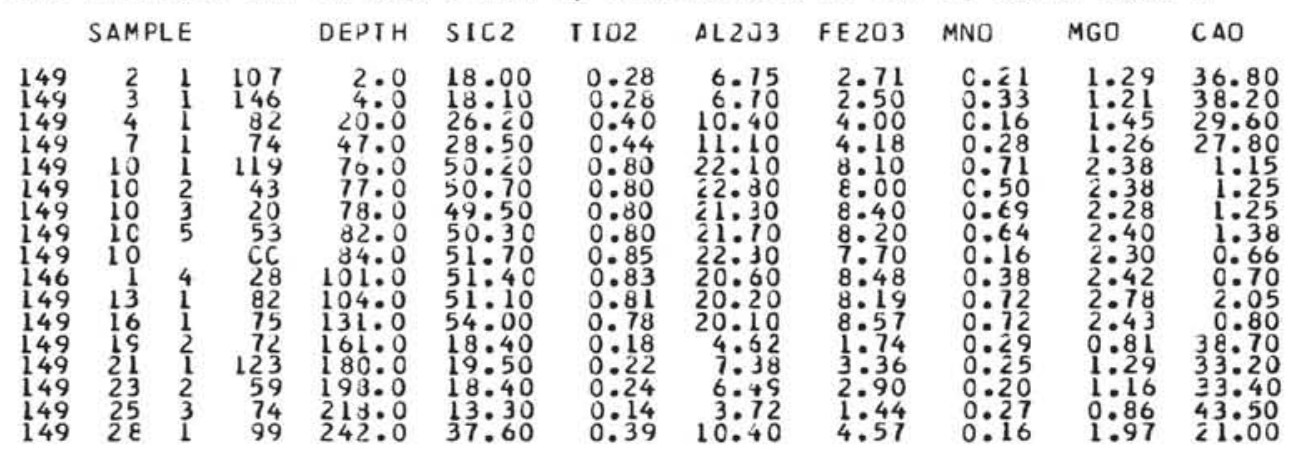

NA 2 C

0.84
2.22
0.79

\subsection{1}

1.41 FCRAN AANAC COLE
1.18 FORAM NANNC CCLE 1.24 FORAM NANAC DOZE
1.68 FCRAM NANAD DOLE 1.31 FCEAM NANNO OCZ 1.23 FCRAM NANAO CCZE 0.99
0.35 FURAM AANNO OCZE $0: 44$ FCRAM NANAC OOZ $1: 05$ MAKL COZE 0.95 SILTY ZEOLITIC CLAY
0.91 SILTY ZEOLITIC CLAY K20 P205 CL LITHCLCGY

$\begin{array}{ll}2: 70 & 0: 17 \\ 1: 82 & 0: 11 \\ 1: 72 & 0: 11 \\ 2: 48 & 0: 15 \\ 1: 42 & 0: 29 \\ 2: 89 & 0: 14 \\ 1: 96 & 0: 53 \\ 1: 45 & 0: 10 \\ 0: 80 & 0: 19 \\ 2: 81 & 0: 12 \\ 2: 14 & 0: 20 \\ 2: 73 & 0: 19 \\ 1: 48 & 0: 15 \\ 1: 11 & 0: 19 \\ 1: 14 & 0: 14 \\ 0: 93 & 0: 14 \\ 1: 23 & 0: 14 \\ 2: 67 & 0: 19 \\ 2: 49 & 0: 19 \\ 2: 98 & 0: 20 \\ 0: 81 & 0: 13 \\ 0: 80 & 0: 13 \\ 0: 76 & 0: 13 \\ 0: 67 & 0: 13 \\ 0: 84 & 0: 15 \\ 2: 22 & 0: 21 \\ 0: 79 & 0.13\end{array}$
$\begin{array}{ll}0.91 & \text { CLAY } \\ 0.57 \text { SLLIY SAND } & \\ 0.64 & \text { SILIY SAND }\end{array}$ 0.80 SILIY CLAY 1.06 CLAY 0.83 MARL FOR-NAN MARL OOZE 0.62 FCR-NAN MARL COZE 0.62 SILIY MARL MUD 0.78 FCRAM CHALK CCZE 0.85 FORAM CHALK CCLE 0.55 SILIY CLAY 0. 49 NANNO MARL MLD 0.53 CALC: MARL COZE 0.54 CALC. MARL DOLE O.54 CALC: MARL COZE 0.59 CLAY MARL CCZE
0.48 CALC. MARL

4.57

$0.86 \quad 43.50$

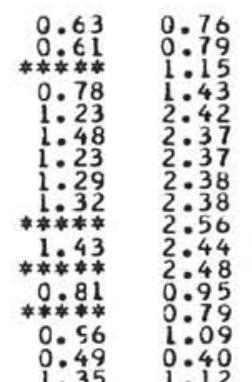

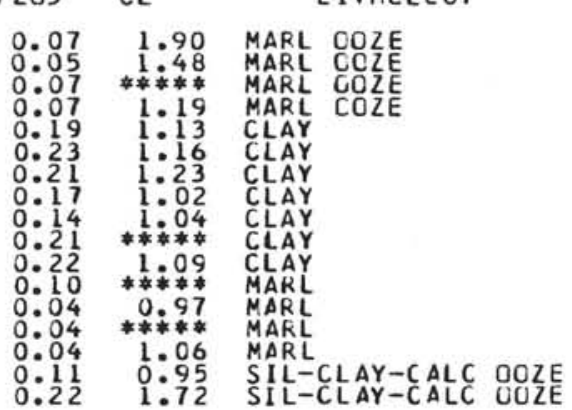

$\mathrm{CL}$

LITHCLCGY

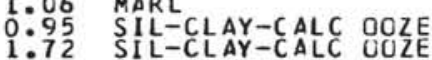


SAMFLE

DEPTH SIO2

$\mathrm{I} 102$ AL2O3 FE2C3 MAC

$\begin{array}{rrrrrr}149 & 29 & 2 & 1184 & 254.0 & 46.50 \\ 149 & 29 & 2 & 149 & 254: 0 & 27: 30 \\ 149 & 30 & 2 & 78 & 263: 0 & 35: 20 \\ 149 & 31 & 2 & 124 & 274: 0 & 53: 50 \\ 149 & 32 & 2 & 123 & 282: 0 & 54: 50 \\ 149 & 33 & 1 & 54 & 289: 0 & 67.60\end{array}$

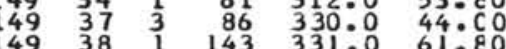

$149 \quad 40 \quad 2 \quad 14 \quad 355: 0 \quad 53: 50$

$\begin{array}{rrrrrr}149 & 42 & 2 & 136 & 367.0 & 50.10 \\ 146 & 3 & & 40 & 383: 0 & 46000\end{array}$

$\begin{array}{llllll}146 & 5 & 1 & 1 & 422: 0 & 57: 50 \\ 146 & 5 & 2 & 29 & 42500 & 66: 30\end{array}$

$\begin{array}{llllll}146 & 7 & 1 & 67 & 440: 0 & 83: 80 \\ 146 & 7 & 1 & 80 & 441: 0 & 56: 70\end{array}$

$\begin{array}{lllll}46 & 7 & C C & 449.0 & 21.60 \\ 146 & 8 & C C & 459.0 & 79.10\end{array}$

\begin{tabular}{llllll}
46 & 10 & 2 & \multicolumn{1}{c}{4} & 470.0 & 81.90 \\
46 & 10 & & $C C$ & 476.0 & 75.30
\end{tabular}

$\begin{array}{lllll}146 & 11 & C C & 485: 0 & 37: 40 \\ 146 & 12 & C C & 494.0 & 61: 80 \\ 146 & 13 & C C & 503.0 & 15.90\end{array}$

$\begin{array}{llllll}146 & 13 & & c C & 503 \\ 146 & 14 & 1 & 95 & 504: 0 & 66: 00 \\ 146 & 14 & 2 & 87 & 506: 0 & 49.60\end{array}$

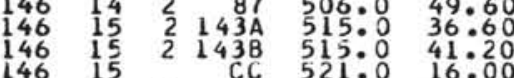

$\begin{array}{llllll}146 & 15 & & \mathrm{CC} & 521: 0 & 16: 00 \\ 146 & 16 & 3 & 9 & 524: 0 & 18: 10 \\ 146 & 16 & 3 & 37 & 525: 0 & 51: 10 \\ 146 & 16 & 6 & 75 & 529: 0 & 52: 10\end{array}$

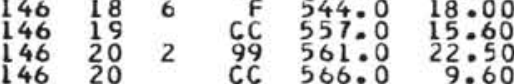

$\begin{array}{llllll}146 & 21 & & C C & 575: 0 & 4.60 \\ 146 & 23 & 2 & 93 & 586: 0 & 43.20 \\ 146 & 23 & 3 & 5 & 587: 0 & 36: 50\end{array}$

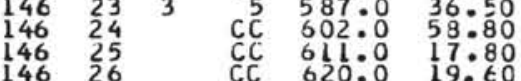

$\begin{array}{lllll}146 & 26 & C C & 620: 0 & 19: 60 \\ 146 & 27 & C C A & 629: 0 & 63.00 \\ 146 & 27 & C C B & 629: 0 & 54 . C O\end{array}$

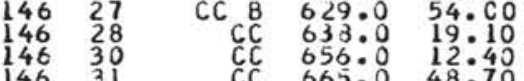

$\begin{array}{lllll}146 & 31 & C C & 665: 0 & 12.40 \\ 146 & 32 & C C & 674: 0 & 15.40 \\ 146 & 33 & C C & 683: 0 & 6.10\end{array}$

$\begin{array}{llllll}146 & 33 & & C C & 683: 0 & 6: 10 \\ 146 & 34 & 1 & 51 & 684: 0 & 55: 30\end{array}$

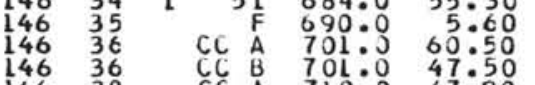

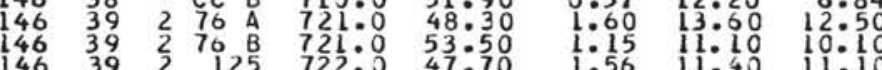

147 :LA

DEPTH SICZ

NG 65 CEG 10 M

उ.2 29.30

$\begin{array}{rrrr}147 & 2 & 1 & 124 \\ 147 & 2 & 2 & 114 \\ 147 & 2 & 3 & 124 A \\ 147 & 2 & 3 & 1248 \\ 147 & 2 & 4 & 80 \\ 147 & 3 & 3 & 87 \\ 147 & 4 & 6 & 85 \\ 147 & 5 & 2 & 89 \\ 147 & 6 & 6 & 100 \\ 147 & 7 & 2 & 84\end{array}$

$\begin{array}{ll}3.0 & 32.10 \\ 3.2 & 50.80 \\ 3.2 & 40.80\end{array}$

$\begin{array}{rr}77: 3 & 44: 00 \\ 17: 9 & 45: 80 \\ 31: 4 & 47: 80\end{array}$

$\begin{array}{ll}34: 4 & 47.80 \\ 34.4 & 44.10 \\ 53.5 & 44.70\end{array}$
MGC

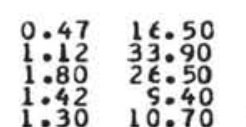

0.09
0.15

NA2C K2O P205

2.10
$1: 11$
$1: 20$
$+* 4+4$
$* * * * * *$ $\begin{array}{lll}1.14 & 5.00 & * * * * * \\ 0.73 & 21.90 & 0.69\end{array}$ $\begin{array}{lll}0.98 & 10: 30 & 0: 55 \\ 0.71 & 19.30 & 0: 74\end{array}$ $0.76 \quad 21.60 \quad 0.55$ $1.87 \quad 23.40$ $4.62 \quad 1.30$ 4.76
1.66
1.89
1.53 1.62 $\begin{array}{ll}2.68 & 1.00 \\ 3.25 & 22.20\end{array}$ $3.11 \quad 5.80$ $4.60 \quad 22.80$ $3.53 \quad 13.30$ $\frac{1}{3} . \frac{2}{6}$ $4.25 \quad 47.50$ 0.8438 .10 $\begin{array}{ll}0.81 & 41.20 \\ 0.79 & 42: 10\end{array}$ $\begin{array}{rr}0.82 & 44.10 \\ 5.12 & 1.80\end{array}$ $4.91 \quad 1.50$ $1.41 \quad 35.10$ $\begin{array}{lll}1.07 & 33.30 & 0: 06\end{array}$ $\begin{array}{ll}1.90 & 16: 40 \\ 0.70 & 17: 90\end{array}$ $0.16 \quad 36.90$ $0.36 \quad 28.20 \quad 0.29$ $\begin{array}{lll}2.74 & 37.20 & 0: 15 \\ 0.39 & 38.70 & 0.01\end{array}$ $\begin{array}{lll}1: 16 & 38: 70 & 0: 40 \\ 0.62 & 15: 20 & 0: 42\end{array}$ $\begin{array}{lll}1.85 & 6: 40 & 0: 57 \\ 0.21 & 19: 50 & 0: 09\end{array}$ $\begin{array}{lll}8.40 & 4.10 & 3: 50 \\ 6.40 & 4.40 & 2: 25\end{array}$ $6.78 \quad 7.20 \quad 1.94$
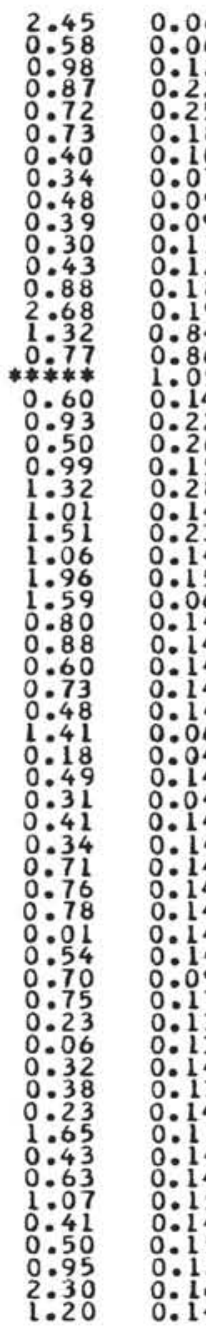

6
5
2
5
8
7
9
1
2
8
4
6
5
4
6
5
4
4
4
5
6
4
4
4
4
4
4
4
4
4
4
4
4
4
7
3
3
4
3
3
4
5
4
5
2
6
4
5
5
5
5
5
5
5

L

LI THCLCGY

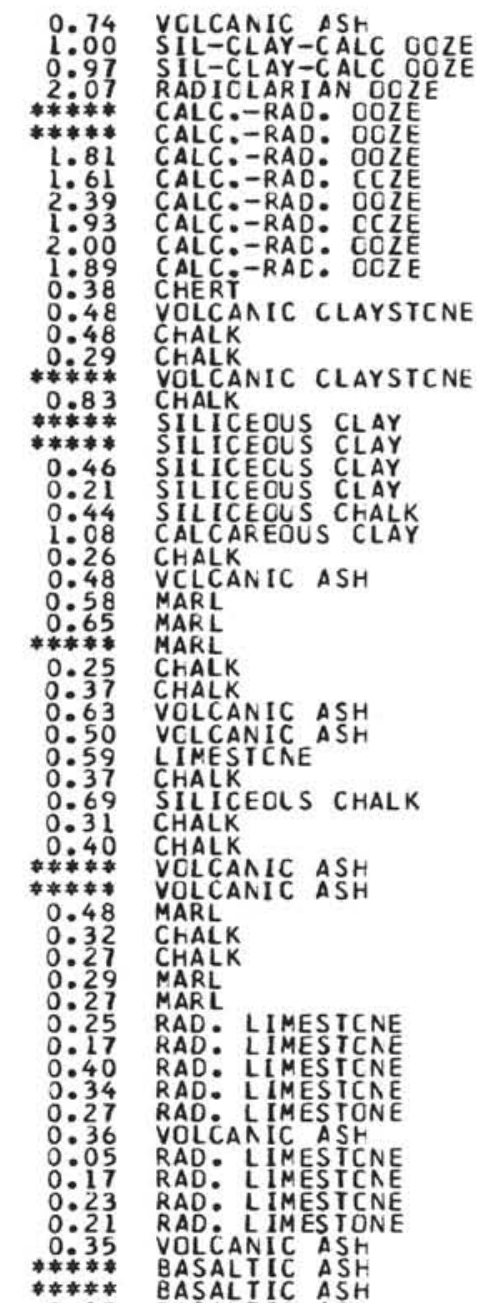

***** BASALIIC ASH
0.15 EASALIIC ASH
0.15 ASALIC ASH

MGO

NA2O

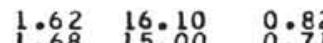

3.8

0.04

1.6

$\begin{array}{ll}1.79 & 4.55 \\ 1.69 & 10.90\end{array}$

$\begin{array}{ll}6.14 & 0.06 \\ 6.76 & 0: 06\end{array}$

$\begin{array}{ll}6.27 & 0.06 \\ 6.25 & 0.16\end{array}$
0.82
$0: 71$
1.83

1.44

1.27
0.79
0.76

0.76 

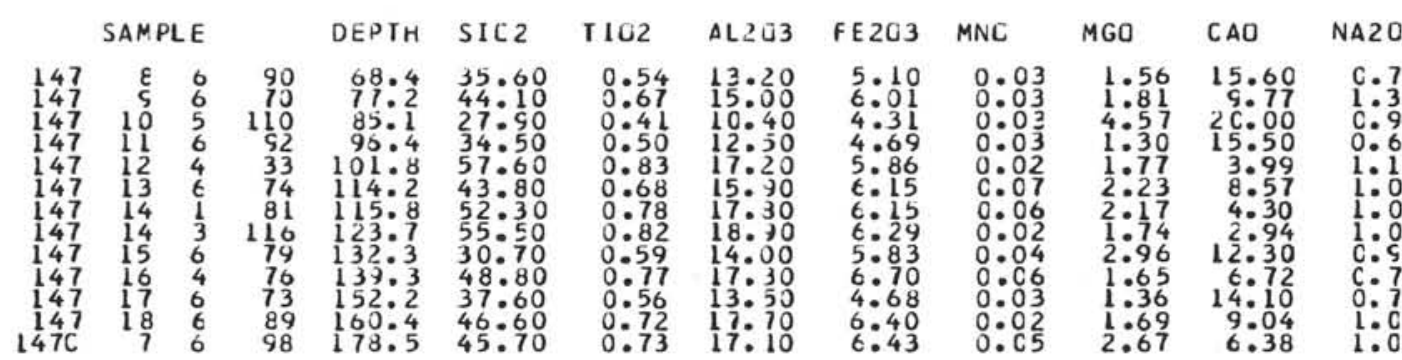

SITE 148:

$$
\text { DEPTH SIUZ TIOZ }
$$

NIN W; DEFIH 1232

$32 \mathrm{M}$ (ANAL. BUDD)

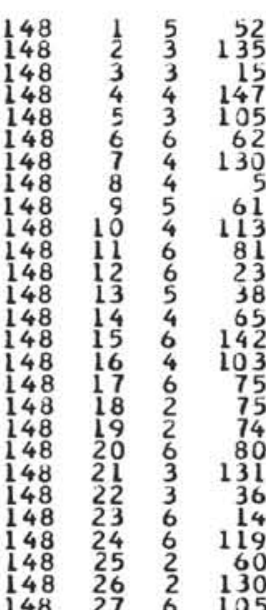

$$
19.0 \quad 43.50
$$

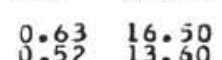

8.85

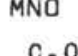

\subsection{3}

$$
\text { MO }
$$

CAO
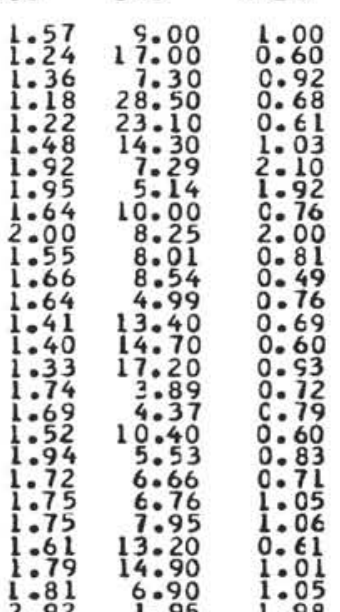

$1: 00$
$0: 60$
$0: 92$
0.68 $\begin{array}{ll}24.0 & 45.80 \\ 33.0 & 25.00 \\ 45.0 & 30.80\end{array}$

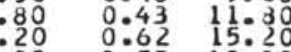

73:0 $46: 20$

$0.75 \quad 18.00$

0.68 17:30

91.0
105.0 49.40 $109: 046: 10$ $125: 0 \quad 43: 60$ 145.0 51.30 $0.67 \quad 17: 80$ $0.62 \quad 15.30$ $0.59 \quad 14.50$ 0.8120 .60 $\begin{array}{llll}175: 0 & 46: 30 & 0: 70 & 17: 30 \\ 184: 0 & 50: 00 & 0: 74 & 19: 5\end{array}$ $\begin{array}{llll}193.0 & 48.90 & 0.70 & 18.30 \\ 203.0 & 47.90 & 0.74 & 18.20\end{array}$ $221: 0 \quad 45.20$ $0.67 \quad 16: 70$ 0.59 16.3

$\begin{array}{ll}4.12 & 0.02\end{array}$

$3.34 \quad 0.04$

$5:$

$6.26 \quad 0.04$

$6.25 \quad 0.04$

$6: 70 \quad 0.04$

6.910 .04

$5: 80 \quad 0.06$

$6.70 \quad 0.05$

$\begin{array}{ll}6.16 & 0.04\end{array}$

$\begin{array}{ll}6.05 & 0.07 \\ 6: 25 & 0: 06 \\ 9.06 & 0.04\end{array}$ 0.61 $1: 10$
$1: 92$
10

2.76

0.81

0.76

0.69

0.73

..79 0.80 0.71 1.06 :. 11

SITE 150: LAT 14 DEG 31 NIA $N$ : NG 69 CEG $21 \mathrm{NIN} \mathrm{W}$; CEPTH $4545 \mathrm{M}$ (ANAL. BUDD)

SAMPLE

DEPTH SIO2

102 AL2O3 FE2C3 MNO

$\begin{array}{rr}7.70 & 0: 12 \\ 10: 70 & 0: 30 \\ 8: 20 & 0: 87 \\ 6: 30 & 0: 39\end{array}$

$\begin{array}{lll}C C & 114: 0 & 42.80 \\ C C & 128.0 & 56: 80\end{array}$

$\begin{array}{lll}0.80 & 22.70 & 7 \\ 0.85 & 21.20 & 10: 70 \\ 0.75 & 20.30 & 8.2\end{array}$

$0.60 \quad 13: 80$

5.80

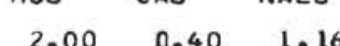
$\begin{array}{rrr}2.00 & 0.40 & 1.16 \\ 2.20 & 0.60 & * * * * 4 \\ 2.60 & 1.10 & 1.89 \\ 2.40 & 10.50 & * * * * *\end{array}$ $\begin{array}{rr}150 & 5 \\ 150 \mathrm{~A} & 2\end{array}$

SITE 151: LAT SAMPLE

DEPTH SIO2

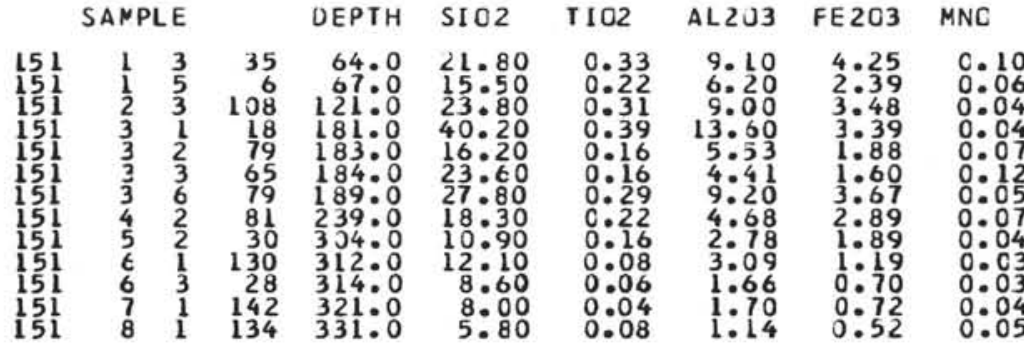

FE 203 MNC

$\begin{array}{ll}4.25 & 0.10 \\ 2.39 & 0.06 \\ 3.48 & 0.04 \\ 3: 39 & 0: 04 \\ 1.88 & 0.07 \\ 1.60 & 0: 12 \\ 3: 67 & 0: 05 \\ 2.89 & 0.07 \\ 1: 89 & 0: 04 \\ 1.19 & 0.03 \\ 0: 70 & 0.03 \\ 0.72 & 0.04 \\ 0.52 & 0.05\end{array}$

MGO

CAO

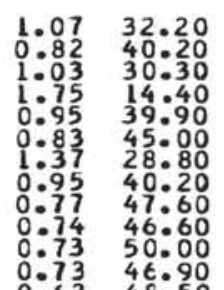

K20 P205

$1: 76$

1.31

2.08
1.85

K20

2.21
$1: 10$
$1: 47$

2.29

$2: 07$
$1: 84$

$\frac{2}{3: 61}$

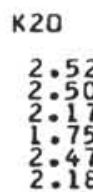

2.52
$2: 50$
$2: 17$
$1: 75$
$2: 47$
$2: 18$

0.20 **** ZEOLIIIC CLAY

0.57
$0: 57$
$0: 45$
$1: 09$
$0: 47$
$0: 55$
$0: 90$
$0: 58$
$0: 69$
$0: 54$
$0: 48$
$0: 49$
0.27

1.51
1.67

$1: 41$

1.89
1.40

.610 .15

1.92
$1: 73$
$1: 77$

2.66

1.80 : 11

1.67

$2: 400.13$

2.52

$2: 24$

P205 CL

$0: 15$

0.38 itia CLAY

0.45 1.31 ZEOLIIIC CLAY

LITHCLCGY

.42 CALCARECLS CLAY 1.29 MARL

1.13 CALCAREOUS CLAY 0.72 CALCAREOUS CLAY 0.69 CALCAREOUS CLAY 1.16 CALCAREOLS CLAY $0: 50$ CALCAREOUS CLAY

CL

LITHCLCGY

2.17 CALCAREOLS CLAY

1.79 CALCAREOUS CLAY $1: 35$ CALCARECUS CLAY 1.66 CALCAREOUS CLAY

$1: 23$ CALCAREOUS CLAY 1.39 HEMIPELAGIC CLAY 0.91 HEMIPELAGIC CLAY 0.84 HENIPELAGIC CLAY 0.80 hEMIPELAGIC CLAY . 0 70 CALCAREOUS CLAY 0.80 CALCARECUS CLAY 0.75 CALCAREOUS CLAY $0: 83$ CALCAREOUS CLAY 0.5

LITHCLOGY

$20 \quad P 205 \quad C L$

$\mathrm{CL}$

LI THCLCGY

$\begin{array}{ll}1.16 & 0.09 \\ 0.75 & 0.09 \\ 1.25 & 0.10\end{array}$ 1.31 MARL OOZE 0.83 CLAYEY CHALK CCZE 0.90 CLAYEY CHALK DOZE 1.43 CLAYEY CHALK COLE 1.23 CHALK 0.99 0.95 CHALK 


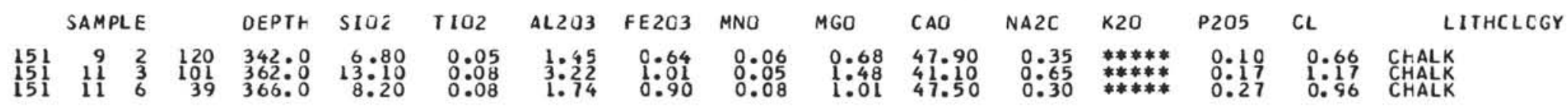

SITE 152: LAT 15 DEG 53 MIN N; LCNG 74 DEG 36 MIN W;DEPTH 3899 M (ANAL BUDD) DEPTH SIO2 TIO2

CAO NA2O

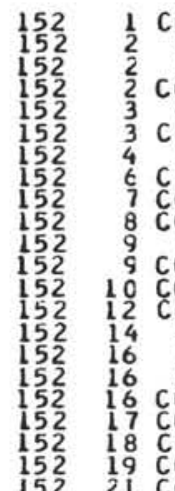

$162.0 \quad 24.80 \quad 0.17$
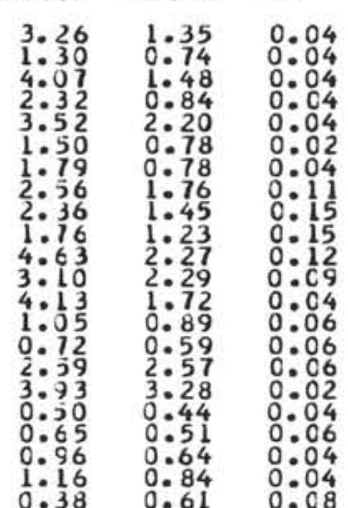

MGO

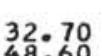

K20

P205 CL

LI THCLCGY

SITE 153: LAT 13 DEG 58 MIN ; LCNG 72 CEG 26 NIN W; DEPTH 3932 M (ANAL. BUDD)

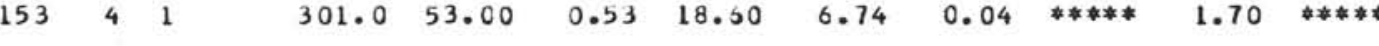

SITE 154: LAT 11 DEG 5 NIN N; LCNG 80 OEG 23 MIN W; DEPTH 3338 M (ANAL. BUDD)

SAMPLE

DEPTH SIO2 TIC2 AL ZC3 FE2O3 MNO

$\begin{array}{lr}154 A & 1 \\ 154 A & 2 \\ 154 A & 3 \\ 154 A & 4 \\ 154 A & 5 \\ 154 A & 6 \\ 154 A & 7 \\ 154 A & 8 \\ 154 A & 9 \\ 154 A & 10 \\ 154 A & 11 \\ 154 A & 12 \\ 154 A & 13 \\ 154 A & 14 \\ 154 A & 15 \\ 154 A & 16 \\ 154 A & 17\end{array}$
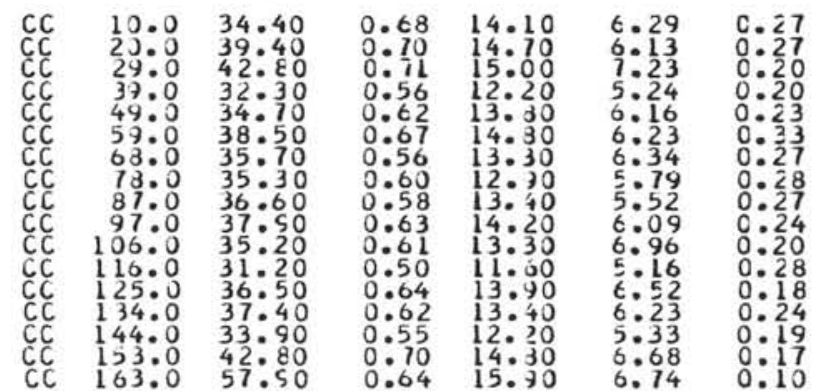

$M G O$
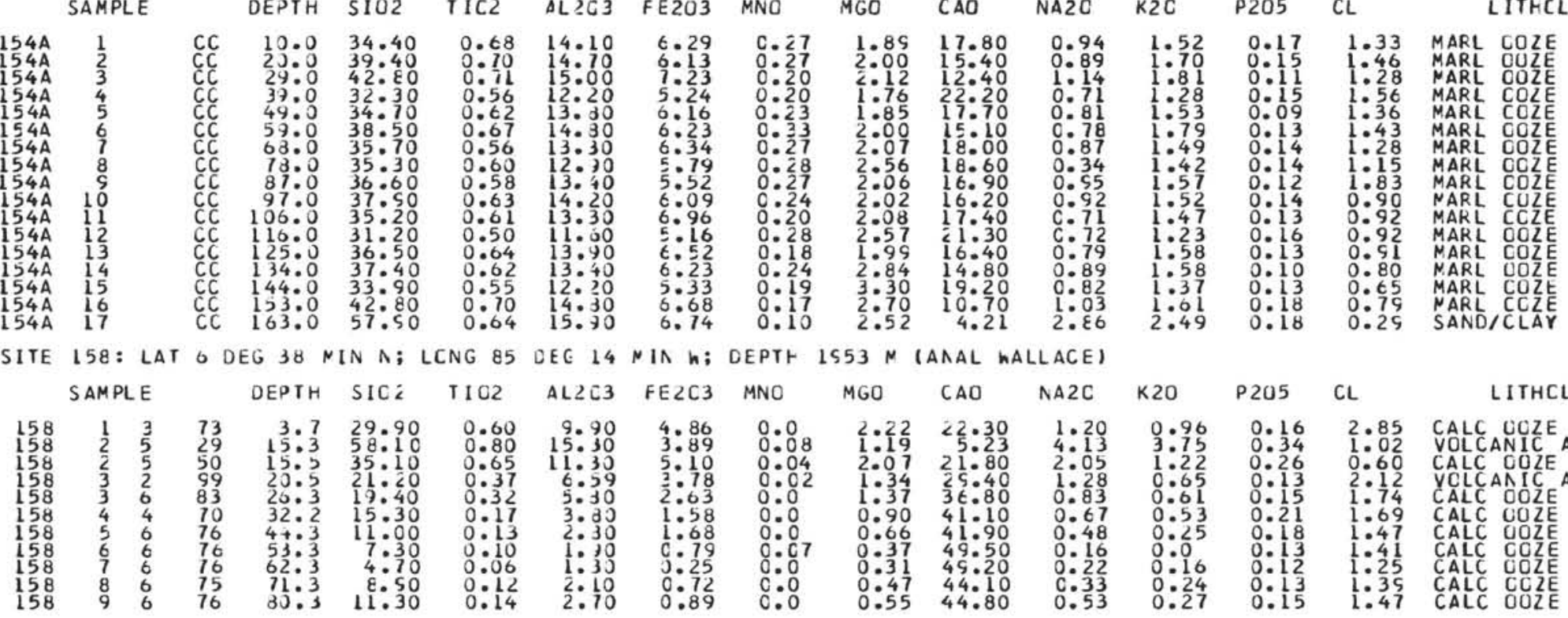

$$
\text { DEPTH SICZ TIC2 }
$$
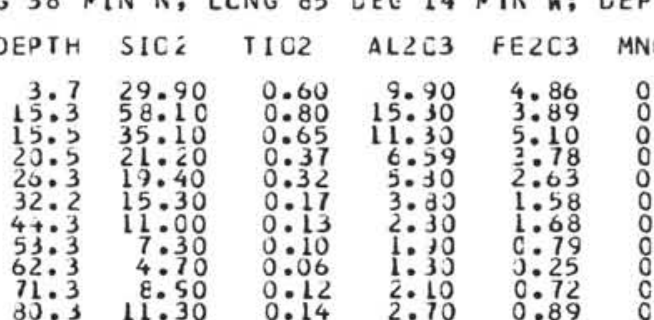

0.60
0.80
0.65
$0: 37$
0.32
$0: 17$
0013
$0: 10$
$0: 06$
$0: 12$
0.14
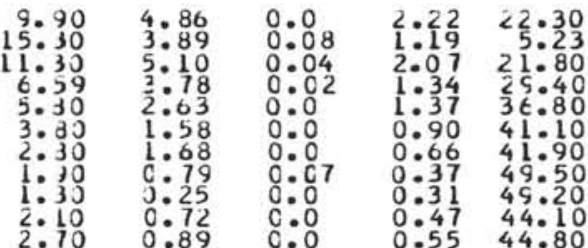

$1: 20$
$4: 13$
$2: 05$
$1: 28$
$0: 83$
$0: 67$
$0: 48$
$0: 16$
$0: 22$
$C: 33$
0.53

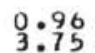

P205 C

$\mathrm{CL}$

LITHCLCGY

$\begin{array}{llll}3: 75 & 0.16 & 2.85 & \text { CALC UCZE HITH RACS } \\ 1.25 & 0.34 & 1.02 & \text { VOLCANIC ASH }\end{array}$

$0.65 \quad 0.23 \quad 0.60$ CALC COLE hITH RACS

$0.53 \quad 0.21 \quad 1.69$ CALC COZE WITH RADS

0.25

$0: 16$

0.18

$1: 47$ CALC DOZE WITH RADS
1.41 CALC COZE WITH RACS

$1: 41$ CALC CCZE WITH RACS

$0.24 \quad 0: 13 \quad 1: 35$ CALC CCZE hITH RACS 
TABLE 3 - Continued

SAMFLE DEPIH SIC2 T102 AL203 FE2 33 MNO 3 - Continued

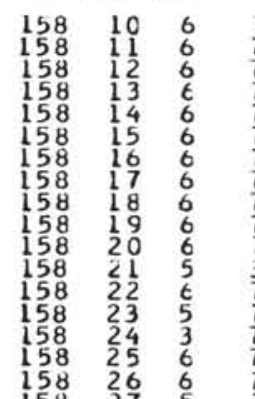

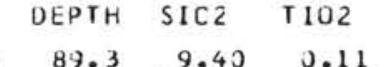

NA2C $\quad \mathrm{K} 2 \mathrm{O}$

P205

$\mathrm{CL}$

LITHCLCGY
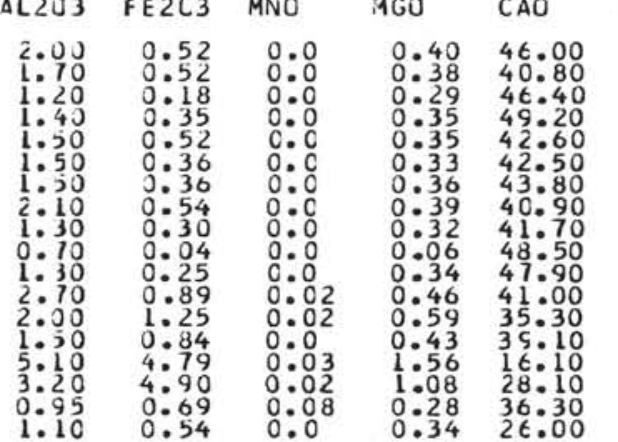

SITE 159; LAT 12 DEG 19 MIN $\wedge$; LCNG 122 CEG 17 NIN W; DEPTH $44 E 4 N$ (ANAL. LI)

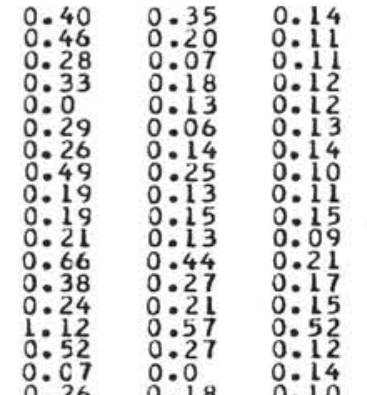

$0: 0$ SAMPLE

DEPTH SIO2 TIO2

AL2U3 FE2O3 MNO

MGO

CAO

NA2O

K20

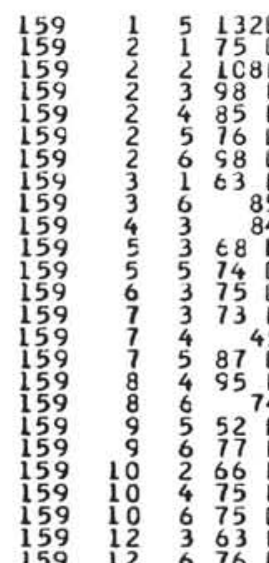

IIF 12 6 76 D $107: 3 \quad 10: 50$
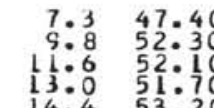

$14: 4$

17

20.6 क $51: 8$

$30: 8$ 24:30

$42.7 \quad 35.40$

59:0 $8: 40$

$78.5 \quad 6.00$
48.8 30.80 0.26

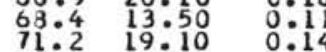

$\begin{array}{rrr}83.2 & 7: 60 & 0: 05 \\ 86.2 & 13: 50 & 0 \\ 8 & 09\end{array}$

$\begin{array}{lll}89: 2 & 18: 40 & 0: 12 \\ 02: 6 & 10: 20 & 0 \\ 0 & 07\end{array}$

SAMPLE

DEPTH $\mathrm{SIO2}$

$\begin{array}{ll}166 & 2 \\ 166 & 2 \\ 166 & 2 \\ 166 & 2 \\ 166 & 2 \\ 166 & 3 \\ 166 & 3 \\ 166 & 4 \\ 166 & 4 \\ 166 & 5 \\ 166 & 5 \\ 166 & 7 \\ 166 & 7 \\ 166 & 8 \\ 166 & 8 \\ 166 & 8 \\ 166 & 9 \\ 166 & 5 \\ 166 & 10 \\ 166 & 12 \\ 166 & 12\end{array}$ $\begin{array}{lll}1.4 & 51.10 & 0.52 \\ 3 & 55.20 & \end{array}$

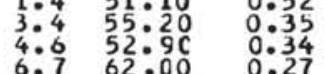

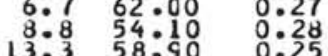

$14: 956: 1000.36$

$23.257: 10$

$\begin{array}{ll}28: 4 & 57: 90 \\ 31: 5 & 42\end{array} 60$

68.950000

$\begin{array}{lll}88: 9 & 60: 30 & 0: 24 \\ 911 & 58: 70 & 0.29\end{array}$

$106: 8$ 54:70 $0: 34$

$\begin{array}{lll}24: 3 & 58: 20 & 0: 26 \\ 162.4 & 67780 & 0: 16 \\ 167.1 & 72: 5 & 0.13\end{array}$
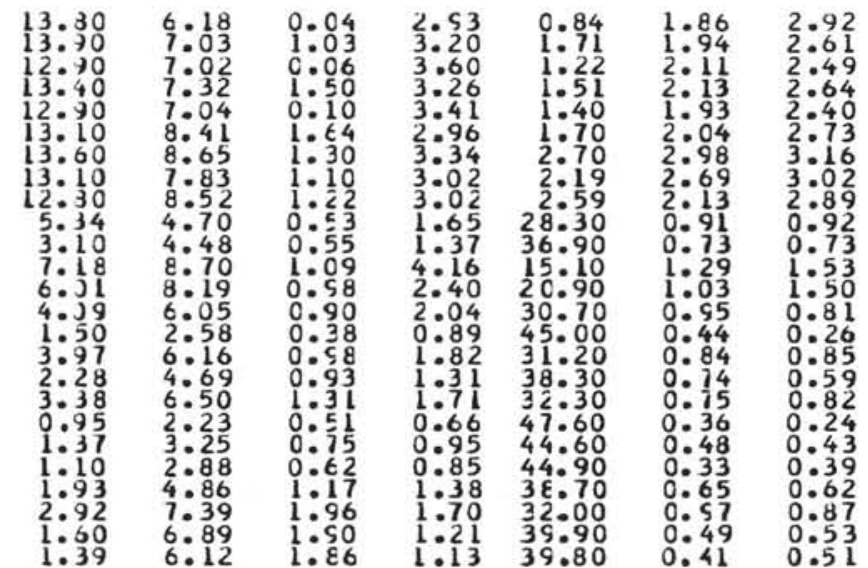

2.92
$2: 61$
2.49
2.64
2.40
2.73

3.16
$3: 02$

2.89
0.92

$1: 53$

0.81

$0: 26$

0.59

0.24

$0: 39$

0.62

0.87

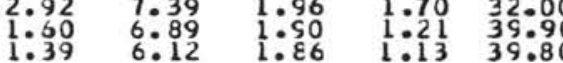

AL203 FE203 MNO MGO CAO NA2O

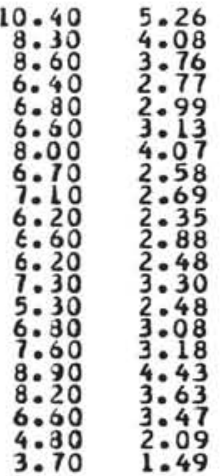

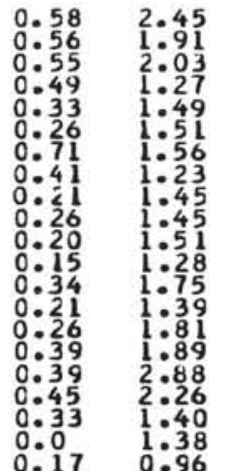

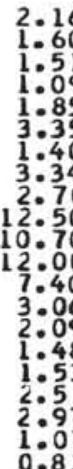

2.04

K20

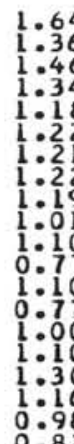

$1: 64$
$1: 36$
$1: 46$
$1: 34$
$1: 18$
$1: 22$
$1: 21$
$1: 22$
$1: 01$
$1: 10$
$0: 77$
$1: 10$
$0: 77$
$1: 00$
$1: 10$
$1: 10$
$0: 98$
$0: 82$
0.69

$1: 41$ CALC OCZE hITH RADS
1.68 CALLC CCZE hITH RACS 1:24 CALC CCZE hIIH RACS 1.75 CALC OUZE hITH RALS $1: 28$ CALC DOZE WITH RACS $1: 41$ CALC CCZE hITH RALS 0.97 CALC CCZE WITH RAOS $1: 18$ CALC OOZE hITH RACS 177 CALC DOZE WITH RADS $2: 40$ CALC CCZE hIIH RADS $2: 01$ CALC COLE hITH RADS
$2: 05$ CALC DOZE WITH RADS

P205 CL LITHOLCGY

$0: 18 \quad 4.14$ CLAY

$0.24 \quad 4: 14$ CLAY

$0.20 \quad 4: 12$ CLAY

$0.21 \quad 3.52$ CLAY

0.20 3.22 CALCAREOUS CLAY

0.19 2.51 MARL

$0: 30 \quad 4: 42$ MARL

0.14

$0: 23$

0.16

$0: 19$

$0: 26$

3.48
$2: 94$
$1: 48$
2.96

$2: 26$ CLAY

1.38

$2: 06$

2.30

MARL
NARL
NARL

MARL

NANNOFOSSIL CHALK

P205 CL

LITHCLCGY

$0: \begin{aligned} & 100 \\ & 0: 93 \\ & 0 \\ & 84\end{aligned}$

ACICLARIAA DOZE

$1: 06$

$0: 91$

0.55

0.45

$0: 75$

0.45

$0: 80$

0.80

0.70
0.44

6.50
$6: 12$
$5: 91$
$5: 91$
$6: 82$
$5: 80$
$6: 52$
$5: 29$
$5: 50$
$4: 99$
$4: 95$
$5: 05$
$5: 48$
$5: 78$
$5: 54$
$5: 35$
$5: 06$
$5: 11$
$5: 33$
4.78

RADICLARIAN DOLE
RADICLARIAN OOZE
RADIOLARIAN OOZLE

RADICLARIAN OCLE

RADIOLAR IAN DOZE

NANNO-RAD DOZE

4:50 NANNO-RAD DOZE

4.95 NANNO-RAD COZE

NANND-RAD COZE

RADIDLARIAN DOZE
RADICLARIAN OOZE

5:35 RADIOLARIAN COLE

$5: 11$
RADICLARIAN CCZE
$5: 33$
RADIDLARIAN DOLE
RADIOLARIAN OCZE 


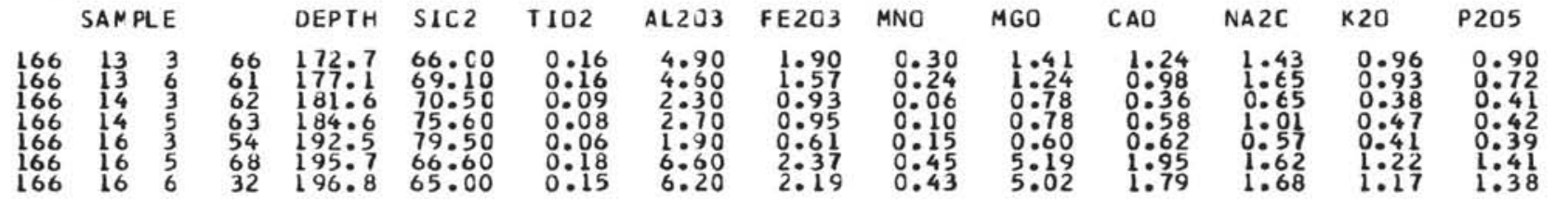

SITE 167: LAT 7 DEG 4 NIN $\Lambda$; LONG 176 DEG 50 MIN W; DEPTH $\equiv 176$ M (ANAL. WALLACE)
DEPTH SIU2 TIO2

\begin{abstract}
AL2J3 FE2O3 MNO
\end{abstract}
MGO

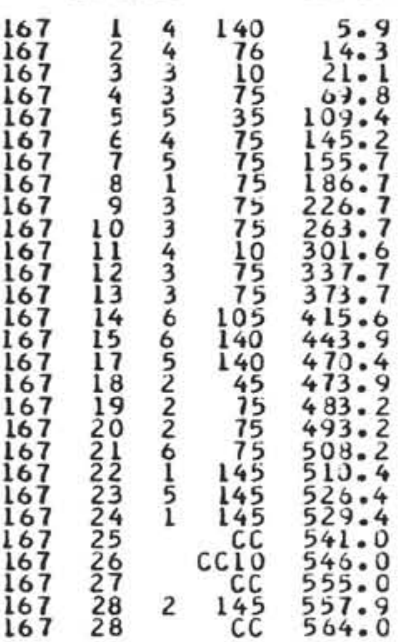

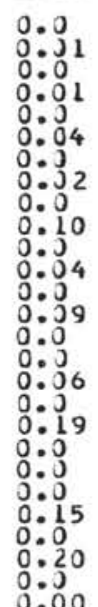

0.15
$0: 000$
0.09
0.06
0.04

0.06
$0: 04$
$0: 03$
$0: 03$

$0: 10$

0.02

0.06

$0: 01$

$0: 01$

$0: 06$

0.16

0.06

0.06

0.04

0.08

0.102

$0: 07$

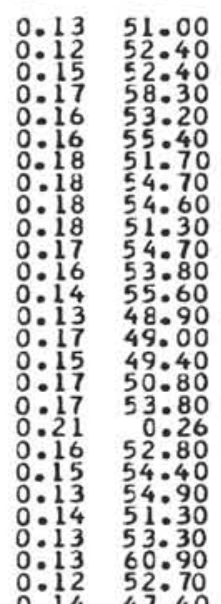

$\begin{array}{ll}0.14 & 47: 40 \\ 0.13 & 49.50\end{array}$
NA2C K20 P205

$\begin{array}{ll}0.07 & 0.03 \\ 0.07 & 0.13 \\ 0.03 & 0.09\end{array}$

$0.01 \quad 0.09$

0.19

$0.03-0.07$

$0: 17 \quad 0: 14$

0.0

$0.11 \quad 0.08$

$0.23 \quad 0.15$

$0.06 \quad 0: 12$

$0: 13 \quad 0: 19$

$0: 10 \quad 0: 14$

$0.25 \quad 0: 05$

SITE 173: 3S DEG 58 MIN N LAT; 125 DEG 27 MIN h LONG; DEPTH 2927 M (ANAL WALLACE) DEPIH $\mathrm{SIO2}$

AL203 FE2O3 MNO MGO

\begin{abstract}
CAO
\end{abstract}

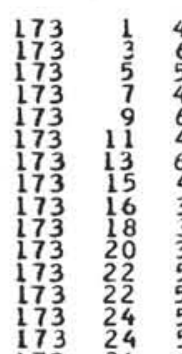

$\begin{array}{lllll}173 & 26 & 5 & 117 & 221 \\ 17 & 7 & 69 & 9 & 90\end{array}$

$0.73 \quad 14.50$

\begin{abstract}
7.07
\end{abstract}

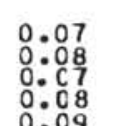

0.07
$0: 08$
$0: 07$
0.08

$6.06 \quad 0.09$

$\begin{array}{ll}7.13 & 0.07 \\ 4: 75 & 0: 10\end{array}$

4.25

2.04

$\begin{array}{lll}0.04 & 2.30 & 5.16 \\ 0: 05 & 1: 06 & 21: 30 \\ 0.05 & 1: 48 & 14: 90 \\ 0.04 & 1.14 & 2.42\end{array}$

$\begin{array}{lll}0.04 & 1.14 & 12.42 \\ 0.04 & 1.05 & 11.20\end{array}$

$4.22 \quad 2: 20$

$2.88 \quad 0.02$

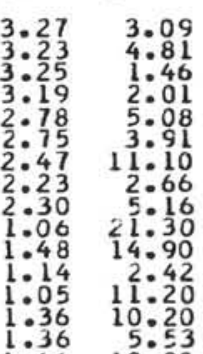

A2C K2O

SITE 178: 56 DEG 57 MIN N LAT; 147 DEG 8 MI

DEPTH SIO2 TIO2

MIN n LCNG; DEP

218 MIANAL WALL ACE)

$\begin{array}{rrrrrr}178 & 2 & 1 & 117 & 7.2 & 65.80 \\ 178 & 4 & 4 & 112 & 29: 6 & 56: 3 \\ 178 & 4 & 4 & 137 & 29: 9 & 57: 5 \\ 178 & 8 & 4 & 86 & 65: 4 & 61: 2 \\ 178 & 12 & 2 & 31 & 97: 8 & 57: 4 \\ 178 & 12 & 2 & 84 & 93: 3 & 56: 0 \\ 178 & 15 & 2 & 50 & 125: 0 & 56: 9 \\ 178 & 20 & 4 & 87 & 173: 4 & 59: 2\end{array}$

$1.00 \quad 0.92$
Cl

4.89 RADICLARIAN CCZE
4.89 RADIOLARIAN OOZE . 4.02 RADIOLAR IAN DOZE 1.50
1.89

$\mathrm{CL}$

LITHCLCGY

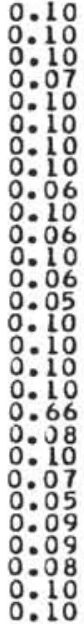

$\begin{array}{ll}1.23 & \text { FCRAM-NANNO OOZE } \\ 1.41 & \text { FORAM-NANNO OOZE } \\ 1.36 & \text { FORAM-AANNO OOZE }\end{array}$ 1.O1 FORAM-AANNO OOZE

:11 FCRAM-NANAC

0.96 FORAM-NANNC DOZE

OS1 FCRAM-AANNC CrALK

105 FCRAM-NANAC CHALK

0.88 FCRAM-NANAC CHALK

1.41 FORAM-NANNO CrALK

1.34 FCRAM-NANNO CrALK
1.12 FORAM-NANNO CHALK

1.23 FCRAM-NANNC CHALK

0.68 RAD-FCR.NAA. CHALK

0 RAD-FCR. NAN: CHALK

$1: 06$
1.04
$10 A D-F C R$ RANAN: CHALK

1.26 RAD-FCR. NAA: CHALK

0.99 RAD-FCR:NAN: CHALK

0.72 RAD-FCR:NAA: CHALK

0.77 RAD-FOR:NAN: CHALK
0.66 RAD-FCR:NAA: CHALK

LI THCLCGY

2.23
$2: 80$
$2: 48$

$\begin{array}{ll}2: 26 & 2: 48 \\ 2.26 & 1: 21 \\ 2.23 & 1.95\end{array}$

$\begin{array}{ll}2: 20 & 2.08 \\ 1: 92 & 1: 83 \\ 2: 00 & 1.68\end{array}$

$\begin{array}{ll}1: 61 & 1: 49 \\ 0.92 & 0: 57\end{array}$

$\begin{array}{ll}1.02 & 0.76 \\ 0.87 & 0.74\end{array}$

0.75
$0: 76$
0.97
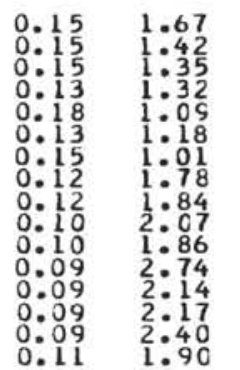

SILIY CLAY

SILIY CLAY

SILIY CLAY

SILIY CLAY TY CLAY

SILTY CLAY

CALC. DIATCMITE

CALC: CIATCMITE
CALC: DIATCMIIE
CALC. CIATCMIIE

2.40 CALC: DIATCMIIE
1.90 CALC. DIATCMIIE

K20 P205 CL

LITHCLCGY

2.44
1.47 SILTY CLAY

0.773 SILIY CLAY

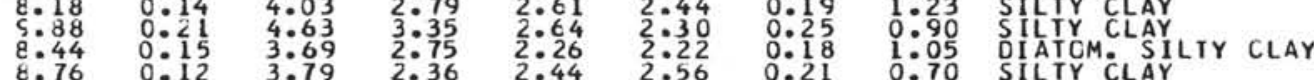

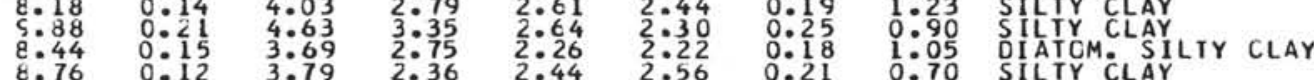


TABLE 3 - Continued
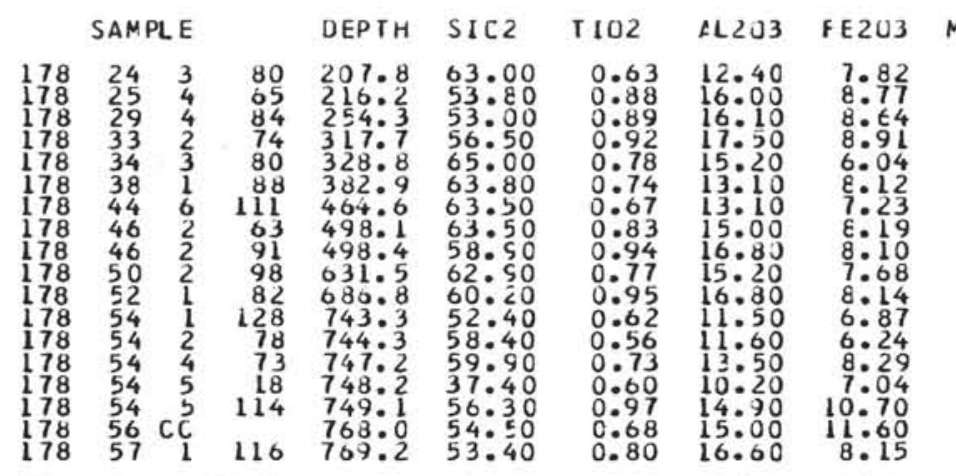

MNU
0.16
$0: 21$
$0: 19$
$0: 29$
$0: 08$
$0: 10$
$0: 28$
$0: 19$
$0: 12$
$0: 09$
$0: 10$
$0: 74$
$0: 92$
$0: 41$
$1: 14$
$0: 39$
0.24
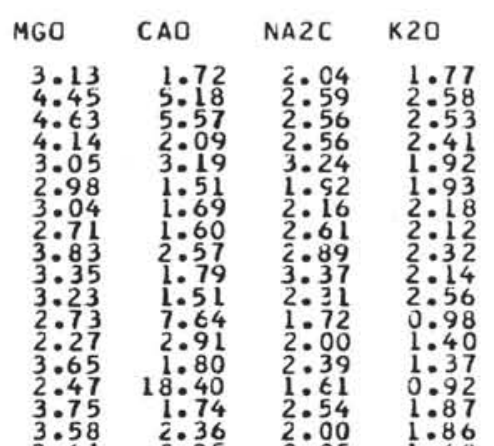

P205

$\mathrm{CL}$

LITHOLOGY

SITE 183: LAT
SAMPLE

DEPTH SIO2

IIU2 AL2O3 FE203 MNC

$47 C 8$

(ANAL LI)
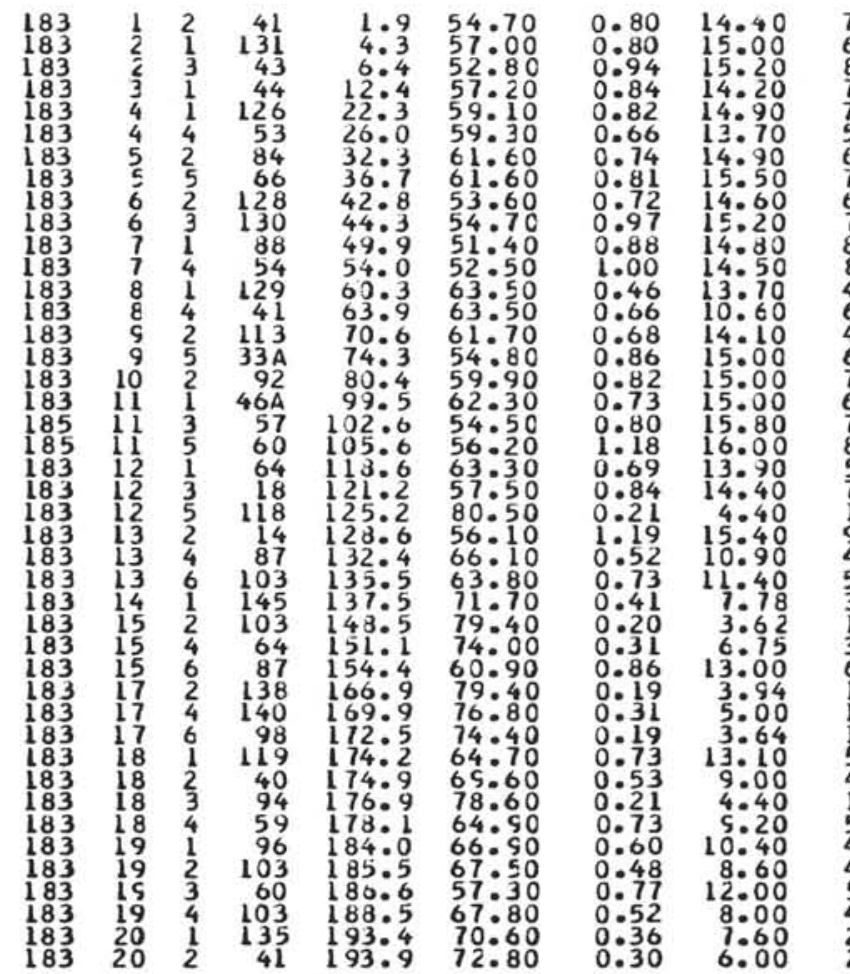

7.60
$6: 76$
$\varepsilon: 28$
$7: 15$
$7: 20$
$5: 25$
$6: 22$
$7: 19$
$6: 95$
$7: 64$
$8: 11$
$8: 70$

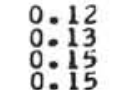

0.12
$0: 13$
$0: 15$
$0: 15$

0.16

$0: 12$

$0: 16$

$1.00 \quad 14.50$

$0.46 \quad 13: 70$

$.68 \quad 14.10 \quad 4.67$

$0.82 \quad 15: 00$

4.03

$0: 100$

0.15

$6: 31 \quad 0: 10$

$\begin{array}{llll}0.80 & 15.80 & 7: 06 & 0: 13 \\ 1.18 & 16.00 & 8.82 & 0: 16\end{array}$

0.6913 .90

0.214 .40

$15: 40$

$0.73 \quad 11.40$

0.20
0.31 $\quad 6.62$

$0.86 \quad 13.00$

$0.19 \quad 3.64 \quad 1.79$

$0.53 \quad 9.00$

$0.73 \quad 5.20$

$0.60 \quad 10.40$

$0.48 \quad 8.60 \quad 4.31$

$0: 1 \frac{1}{12}$

.06 $0: 16$

5.72

$1.81 \quad 0.14$

0.14

$2: 03$

$2: 03$
$0: 11$
$0: 13$

0.1331 .44

CAO NA2C

K20

P205 CL

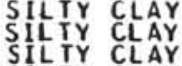

$0: 063$ StITY CLAY

0.82 DIATCMACECLS CLAY

$0.14 \quad 1: 15$ CLAYEY DIATCM DOZE

SLAYEY DIA

0.64 SILAYCLA

0.53 CLATOMITE

0.40 CLAYSICNE

0.54 CLAYSTN

0.52 CALC. CLAYSTONE

0.40 CLAY SHALE

$0: 16$

$\begin{array}{llll}2.76 & 2.51 & 3.07 & 2.01 \\ 2: 80 & 2: 65 & 2: 65 & 2: 10\end{array}$

(a)

0.18 1.42 SILICEOUS CLAY $\begin{array}{llll}2.56 & 4.23 & 2: 93 & 1.35 \\ 2.08 & 2.97 & 2: 87 & 2.00\end{array}$

$2.65 \quad 2.27$

$2.09 \quad 2.34$

2.72
3.27

2.:34

4.52
2.48
3.02

2.82

$\begin{array}{ll}3.26 & 5: 00 \\ 1.43 & 1: 95\end{array}$

$3: 02$

$1: 34$

1.88

$1: 91$
2.45
3.85

$3.49 \quad 3.47$

2.30
2.71
2.43

$2: 59$

3.: 17

2.038

$\begin{array}{ll}2.99 & 2027 \\ 2.72 & 5.08\end{array}$

$2: 69$

$2: 01$

$2: 52$

$0: 3 \frac{1}{3}$

3. 50

$1: 17$
$1: 37$
$1: 97$

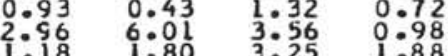

$\begin{array}{llll}1: 81 & 3: 02 & 2: 87 & 1: 33 \\ 1: 03 & 1: 39 & 1: 70 & 1: 23\end{array}$

$\begin{array}{llll}0.73 & 0: 48 & 1: 77 & 0.63 \\ 0.03 & 1: 20 & 1: 64 & 1: 18\end{array}$

$\begin{array}{llll}2.09 & 3.80 & 2: 77 & 1: 62 \\ 0.65 & 0.36 & 1: 17 & 0.90\end{array}$

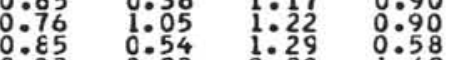

$\begin{array}{llll}.03 & 2: 93 & 3.28 & 1.49 \\ 044 & 1: 95 & 2: 21 & 1: 31\end{array}$

$\begin{array}{llll}.79 & 3.31 & 2.10 & 0.99\end{array}$

$\begin{array}{llll}10 & 2.07 & 2: 94 & 1.68 \\ 1.54 & 1.93 & 2.28 & 1.20\end{array}$

.68
1.20
1.57
1.24
1.08

0.16

48 VOLCANIC ASH

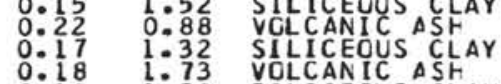

$\begin{array}{lll}0: 10 & 1: 30 & \text { SILICEOLS CLAY } \\ 0.14 & 2013 & \text { SILICECUS CLAY }\end{array}$

$0: 16$ 1:16 VOLCANIC ASF

0.17 1:30 SILICEOUS CLAY

0.2101 .45 SILICECLS CLAY

0.14 1:53 SILICEQUS CLAY

0.042 .25 SILICEOUS CLAY

$\begin{array}{lll}0.12 & 2: 43 & \text { SILICECLS CLAY } \\ 0.14 & 2: 03 & \text { SILICEOS CLAY } \\ 0 & 2 & 0\end{array}$

2.78 SILICECUS CLAY

$0.06 \quad 2: 20$ SILICEOUS CLAY

$0.03 \quad 2: 75$ SILICECUS CLAY

$0.06 \quad 2: 31$ SILICEOUS CLAY

0.10 2.5

SILCEOS CLAY

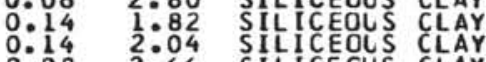

0.08

$2: 66$ SILICECUS CLAY

0.08

$2: 37$

SILICEOUS CLAY

SITE 191: LAT 56 DEG 57 MIN $\wedge$; LCNG 168 DEG 11 MIN E; CEPTH 3854 M(ANAL WALLACE)

SAMPLE

DEPTH $\mathrm{SIO}$

TIO2 AL203 FE203 MNC

MGO CAO

P205 CL

LITHCLOGY

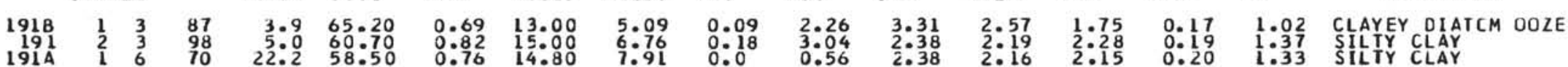




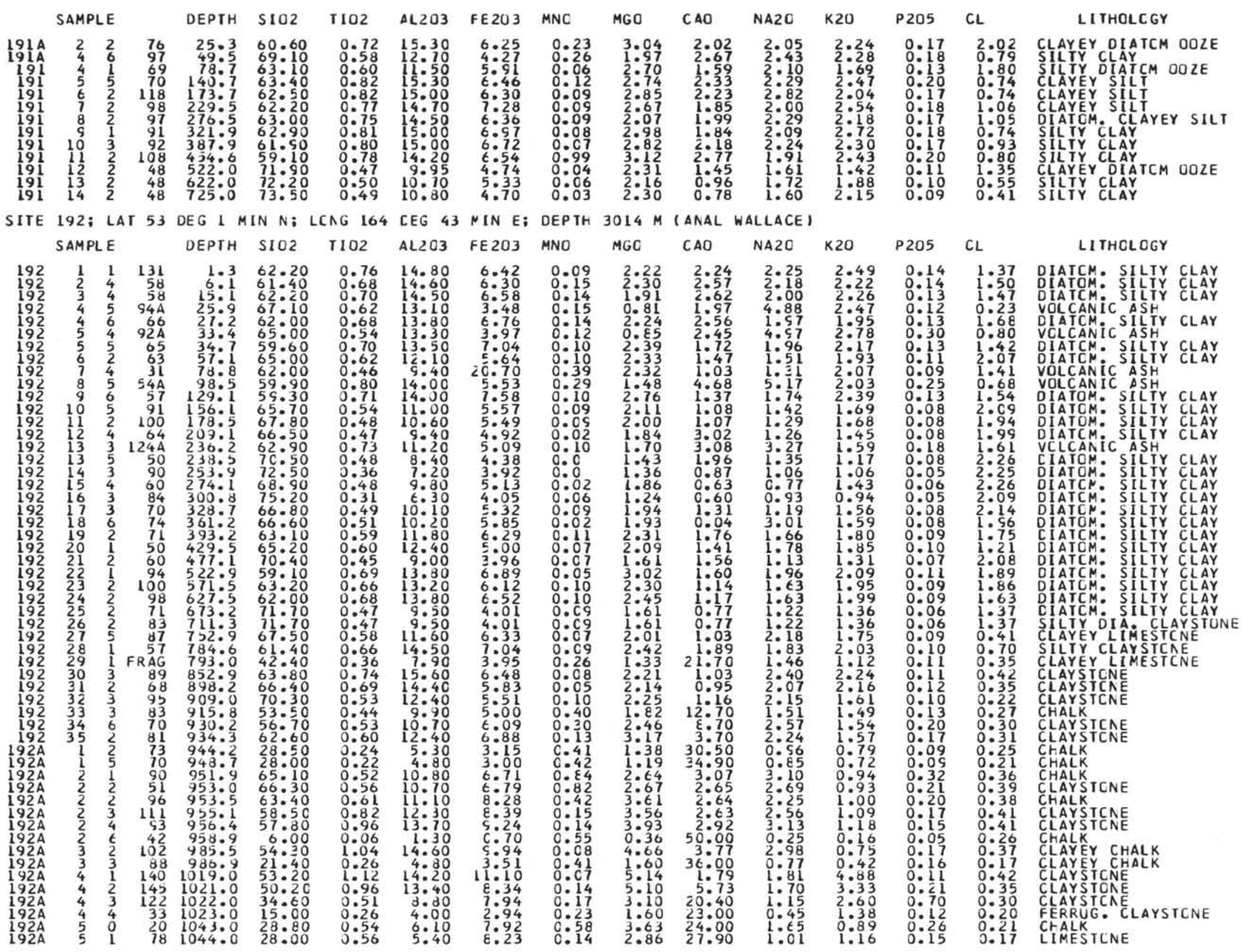


SITE 20 ; LAT 32 UEG I NIN S; LCNG 165 CEG 27 MIN E; DEPTH 3196 M (ANAL WALLACE)

SAMPLE

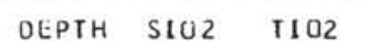

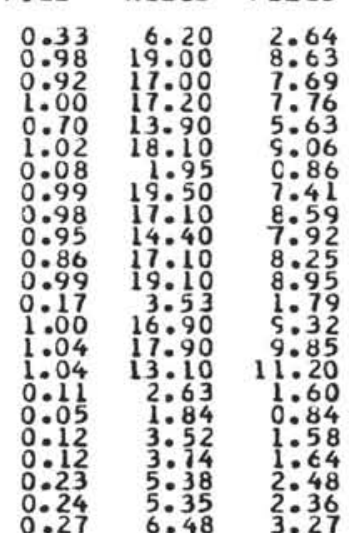

0.28
$0: 13$
$0: 10$
$0: 10$
$0: 30$
$0: 11$
$0: 15$
$0: 07$
$0: 10$
$0: 18$
$0: 10$
$0: 10$
$0: 23$
$0: 21$
$0: 35$
$0: 25$
$0: 15$
$0: 19$
$0: 18$
$0: 18$
$0: 17$
$0: 15$
0.13

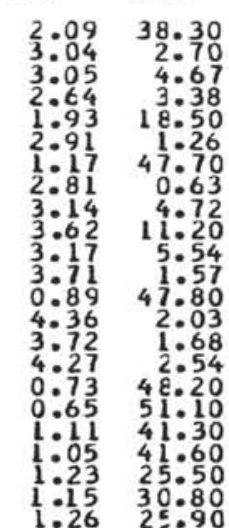

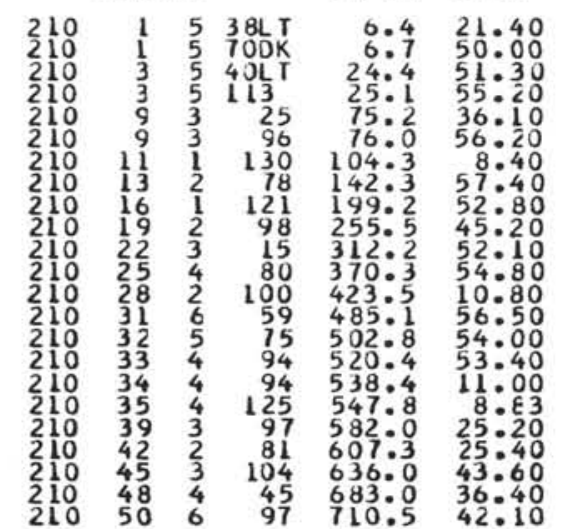

0.27

NA2C $\mathrm{K} 20$

P205 CL

0.08
0.08
0.09

1.05

$0.39 \quad 0.45$

0.420 .41

0.21

$0 .: 22$

$0: 19$

LITHCLCGY

FERAM-AANO OOZE
FGRAM-NANAO CCZE ECRAM-NANAC

\section{O2 FCRAM-NANND}

.07 FORAM-NANAO

04 FORAM-NANNO OOLE FCRAM-NANNO OOZE FCRAM-NANNO OOZE FCRAM-NANNO FORAM-NANNO CCZE FORAM-NANAO OOZE FOKAM-NANNO OOZE

0.85 FORAM-NANN DUZE

0.54
$0: 55$
0.82 CLAYEY CALC: COLE COAY CALC. COZE .75 CLAYEY CALC. CCZE 0.64 CLAYEY CALC: COZE SLAYEY CALC D OOZE SIL-CLAY-CALC OOZE CLAYEY CALC. COZE CLAYEY CALC: COZE SIL-CLAY-CALC OOLE SIL-CLAY-CALC SIL-CLAY-CALC OCZE P205 CL 0.72
$1: 69$
$1: 98$
$1: 88$
$1: 67$
$1: 81$
$0: 24$
$2: 45$
$2: 26$
$1: 48$
$2: 32$
$2: 55$
$0: 40$
$2: 12$
$2: 18$
$1: 86$
$0: 27$
$0: 14$
$0: 52$
$0: 55$
$0: 82$
1.85

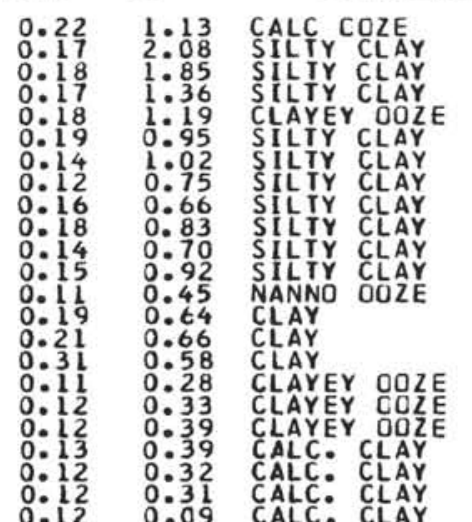


SITE 213: LAT 10 DEG 13 MIN S; LONG 93 CEG 44 MIN E; DEPIH 5611 M (ANAL LII

SAMPLE DEPTH SICZ TIO2 AL203 FE2O3 MNO
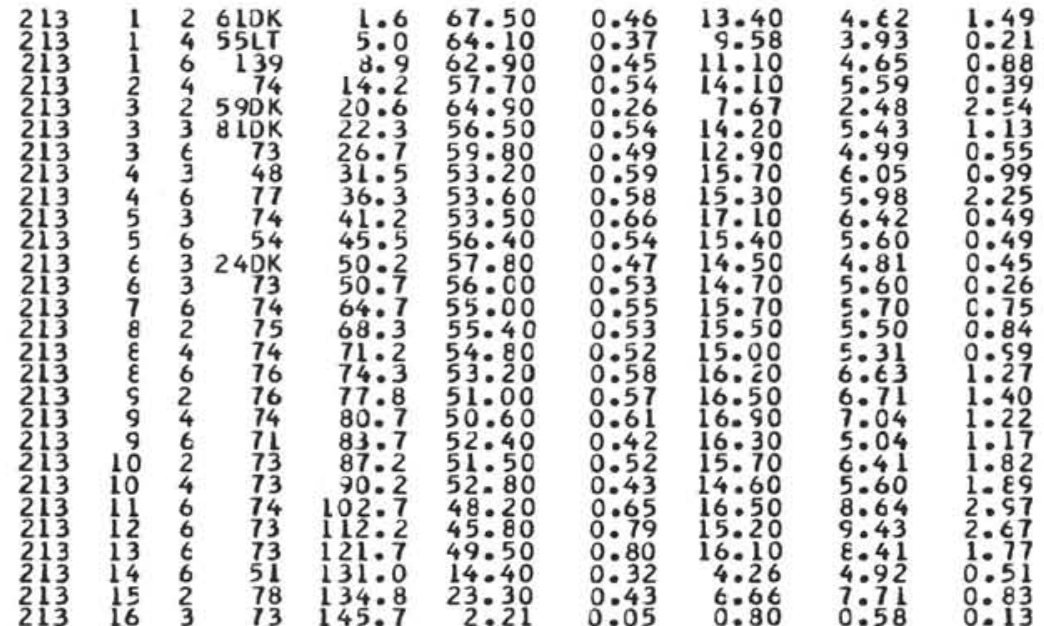

MGO

CAO NA2O

K20

P205

CL

LITHOLCGY

SITE 214: LAT

DEG 20 MIN S; LCNG 88 CEG 43

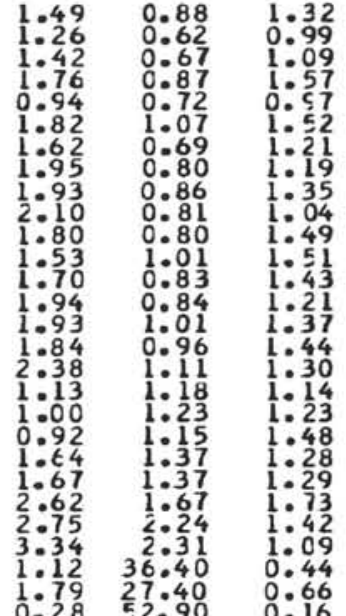

1.90
1.52
1.87 $\frac{1}{2} .48$ 1.65 $1: 94$

AL2O3 FE2 203 MNC

$1665 \mathrm{~N}$ (ANAL WALLACE)

2.36

1.87

SILICEOUS CLAY 4.84 RAD-DIAICM OOZE 5.07 RAD-DIATCN OOZE 4.54 RAD-DIATOM 4.41 3.68 - DAICM COZE 作 3.98 RAD-DIATCM UOZE 4.15 CLAYEY SILIC. OOZE 4.02 SILICEOUS CLAY 3.55 SILICECUS CLAY 3.32 CLAY ZELIIIC CLAY 4.04 ZEGLITIC CLAY 3.60 CLAY 2.29 ZEOY MN-RICH CLAY 2.96 ZEOL. MN-FE CLAY 2.22 ZEOL: MN-FE CLAY $1: 41$ MA-FE CLAY

SAMPLE

DEPIH SIOP TIO2
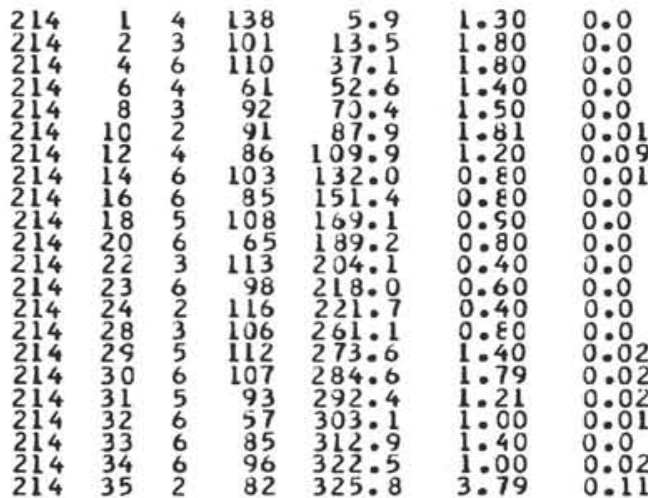

$0.22 \quad 0.04$

0.04
$0: 12$
$0: 30$
$0: 22$

0.03

MGO

NA2C K2O

P205

0.05

$0.24 \quad 53.30$

Cl

1.29 FORAM-NANNO OOZE
1.25 FORAM-NANAO OOZE 1.23 FCRAM-NANAC

.30 FORAM-NANNO OOZE 1.40 FORAM-NANAC OOZE .25 FCRAM-NANAC

$1: 14$ FCRAM-NANNC OCZE

$1: 14$ FORAM-NANNO OOZE

0.70 FORAM-NANNO OOZE

0.73 FORAM-NANNO OOZE

. NANAO CCZE

O NANN COZE

1.05 NANAO OOZE

(1) NANAC DOZE

0.64 NANNO OCZE

SITE 217: LAT \& DEG 56 NIN ; LCNG 90 CEG 32 NIN E; DEPTH $3 C 20$ M (ANAL LI)

SAMPLE

DEPIH SICZ

T IO

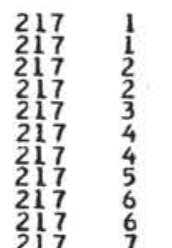

$\mathrm{MGO}$

CAO

NA2C

K20

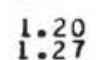

P205

LITHCLCGY

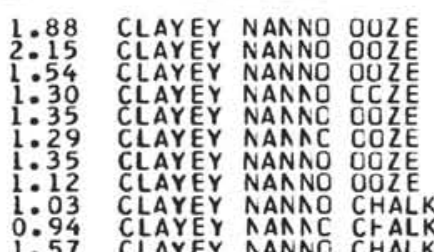


SITE 218: LAT a DEG 1 MIN N; LUNG 86 DEG 17 MIN E; DEPTH 3759 M (ANAL LI)
SAMPLE

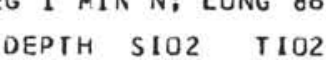

MGO

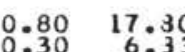

$\epsilon \cdot 68$
$3: 18$

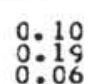

$0.72 \quad 15: 00$

$0.91 \quad 19: 10$

7.80

6.89
4.53

$0: 11$

$\begin{array}{ll}2: 99 & 2.79 \\ 1: 59 & 31: 40\end{array}$

0.08

7.39

4.29
6.76

$186: 0$

$\frac{2}{3} 73^{117}$

$\begin{array}{ll}86.7 & 65.20 \\ 187.7 & 53.5\end{array}$

$0.86 \quad 18.40$

$0.74 \quad 15: 80$

$0.85 \quad 18.70$

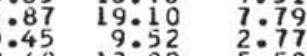

0.16

$\begin{array}{ll}2.77 & 0: 11 \\ 5.50 & 0.08\end{array}$

$\begin{array}{ll}5.50 & 0.18 \\ 6.55 & 0.14\end{array}$

$\begin{array}{ll}4.20 & 0: 10 \\ 4.05 & 0: 10\end{array}$

$\begin{array}{ll}4: 34 & 0.22\end{array}$

2.43
8.20

6.16

6.07 0.11

$0: 16$

$\begin{array}{ll}5.64 & 0: 10 \\ 6.55 & 0: 20\end{array}$

3.70
2.73

$\begin{array}{ll}3.01 & 2.46 \\ 2.38 & 3.52\end{array}$

$\begin{array}{rr}2.65 & 3.25 \\ 3: 03 & 2.16 \\ 1.92 & 26.10\end{array}$

$\begin{array}{rr}3.01 & 26.109 \\ 0.33 & 5.84\end{array}$

$3.31 \frac{1}{3} \quad 2.35$

1.84

$2.13 \quad 30.72$

$\begin{array}{ll}1.67 & 4.87 \\ 3 & .80\end{array}$

$\begin{array}{ll}.13 & 3.58 \\ .83 & 40.20\end{array}$

$\begin{array}{ll}2: 24 & 2: 92 \\ 2: 80 & 4.09\end{array}$

$2.83 \quad 5.27$

$574.8 \quad 55.30$

$0.8219: 10$

6.19

$0: 34$

ONG 61

CEE $31 \mathrm{NIN}$ DEPTH SICZ TIOZ

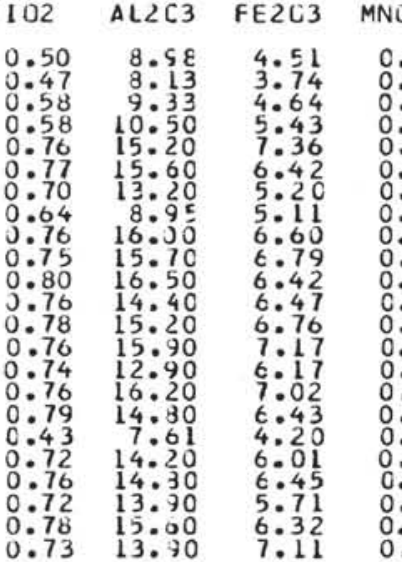

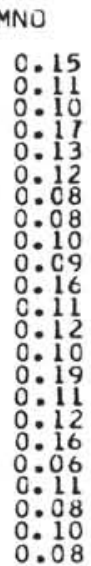

SITE 222: LAT
SANPLE
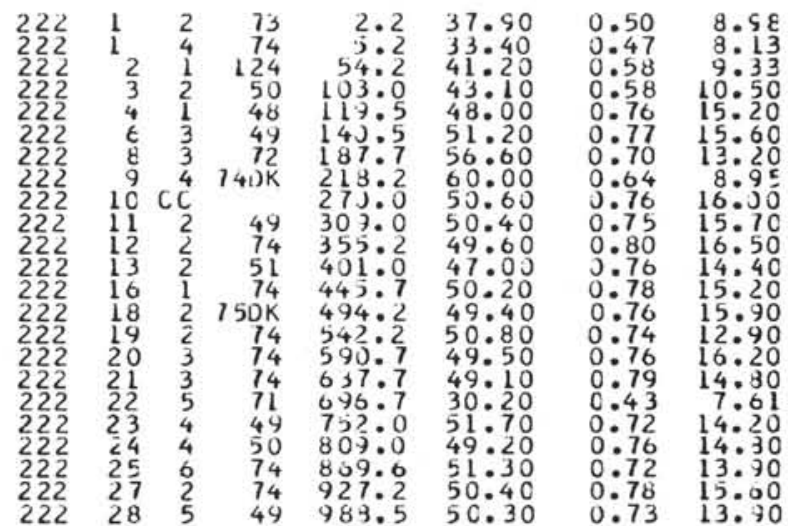

$\begin{array}{lll}74 & 542: 2 & 50: 80 \\ 74 & 590: 7 & 49: 50 \\ 74 & 637: 7 & 49: 10 \\ 71 & 696: 7 & 30: 20 \\ 49 & 757: 0 & 51: 7 \\ 50 & 809: 0 & 49: 20 \\ 74 & 809: 6 & 51: 30 \\ 74 & 927: 2 & 50: 40 \\ 49 & 989: 5 & 50: 30\end{array}$
$0.55 \quad 11.40$

$\begin{array}{ll}1.88 & 22.60 \\ .02 & 5.93\end{array}$

$.19 \quad 4.00$
3.10

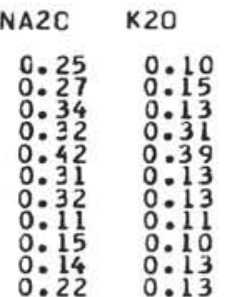

P205

0.08 0.08

NA2 C $\quad \mathrm{K} 2 \mathrm{O}$

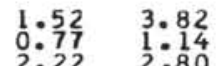

$\begin{array}{ll}1: 76 & 3: 13 \\ 1: 59 & 3: 84 \\ 1: 66 & 3: 55 \\ 1: 26 & 3\end{array}$

\begin{tabular}{ll}
$1: 66$ & $3: 55$ \\
$1: 84$ & $2: 98$ \\
\hline & 3
\end{tabular}

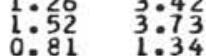

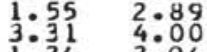

$1: 17 \quad 4: 06$

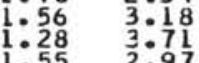

$1: 06 \quad 4: 01$

$: 30 \quad 3: 22$

$.40 \quad 3.28$

: 03 1.24

1.24
$3: 39$
2.28

$\mathrm{CAO}$

NA2C

K20

$0.14 \quad 0.6$

$0: 14 \quad 0.61$

0.14

0.64

$C L$

0.66

LITHOLOGY

CLAYEY NANAO CFALK

CLAYEY AANNO CHALK

NANNO CHALK

ANNO CHALK

AANNO CHALK

CLAYEY NANNO CHALK

P205 CL

LITHCLOGY

$0: 14 \quad 0.91 \quad$ CLAYEY SILT

$0: 14 \quad 1: 46$ SANDY SILT

0.16 0.95 CLAYEY SILT

0.78 CLAYEY SILT

0.15 1.49 CALC CLAYEY SILT

0.08 0.48 VLAYEY SILT

$\begin{array}{lll}14 & 0.63 & \text { CLAYEY SILT }\end{array}$

0.150 .39 CALC SILTY CLAY

0150045 SANDY SILT

$0.14 \quad 0.56$ SILTY CLAY CLAY

$0: 18 \quad 0.71$ SANDY SILT

0.57 CLAY

0.150 .56 SIL

0.57 CLAYEY SILT

$\begin{array}{lll}0.18 & 0.44 & \text { DCZE } \\ 0.13 & 0.52 & \text { SANDY SILT } \\ 0.24 & 0.47 & \text { SILT }\end{array}$

$205 \mathrm{CL}$

1017 SILTY CLAYEY OOZE 0.50 SILTY CLAYEY DOZE 1.18 SILIY CLAY CLAY 0.70 CALC SILIY CLAY 0.43 CALC SILIY CLAY 0.51 CALC SILIY CLAY 0.63 CALC SILIY CLAY 0.69 CALC SILTY CLAY 0.63 CALC SILIY CLAY 0.48 CALC SILTY CLAY 0.38 CALC SILIY CLAY 0.50 CALC SILIY CLAY 0.48 CALC SILIY CLAY 0.32 CALC SILIY CLAY 0.32
0.33 CALC SILIY CLAY 
DEPIH $\mathrm{SIC2}$

I 102

$\triangle L 2 C 3 \quad F E 2 C 3$ MNC

MGC

$C \triangle O$

NA2C

2.20
2.51
1.81
2.43

P205

0.17

$\mathrm{CL}$

LITHCLCGY

$\begin{array}{rrrrrr}222 & 29 & 4 & 740 K & 1046.2 & 40.50 \\ 222 & 31 & 6 & 74 & 1134: 2 & 51: 30 \\ 222 & 32 & 2 & 74 & 1163: 7 & 38: 00 \\ 222 & 33 & 3 & 50 & 1214: 5 & 52: 10 \\ 222 & 34 & 6 & 75 & 1266: 3 & 34: 20 \\ 222 & 36 & 3 & 74 & 1293: 7 & 50: 9 C\end{array}$

$0.52 \quad 10: 50$

$6: 06$
$4: 80$
6.36

$\begin{array}{lll}0 & 13 \\ 0 & 10 \\ 0 & 36 \\ 0 & 23\end{array}$

$\begin{array}{rr}3.63 & 8: 54 \\ 3.57 & 15: 50 \\ 4.40 & 8.11\end{array}$

0.25
1.25
1.35

2.045

SITE 231: LAT
SAMPLE DEPTH SIU2 TIU2

CEG 15 NIN E; DEPTH $2 L \leq 2 \mathrm{~N}$ (ANAL LI)
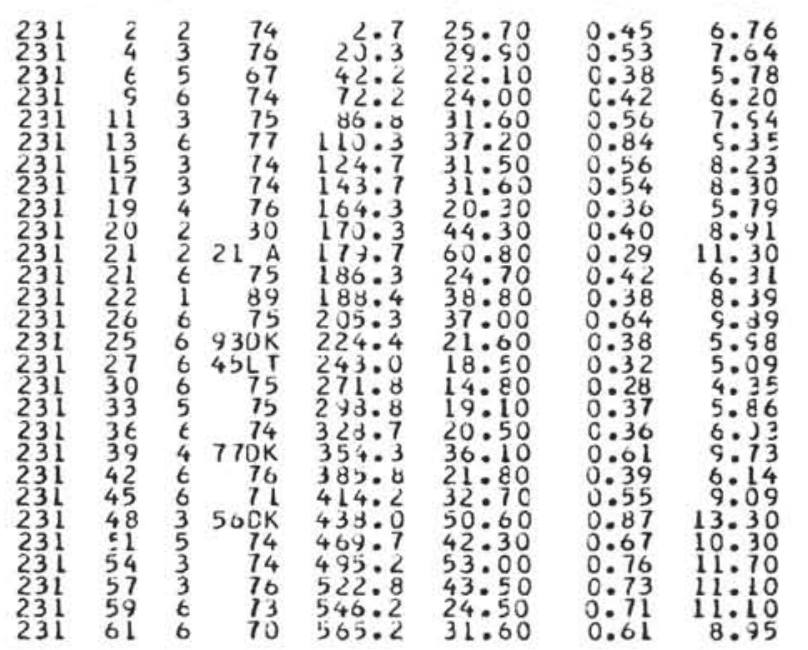

FE203 MNO

$M G O$

CAO

$\mathrm{NA} 2 \mathrm{O} \quad \mathrm{K} 2 \mathrm{O}$

0.18

$\begin{array}{ll}0.34 & \text { CALC SILIY CLAY } \\ 0.29 & \text { CALC SILYY CLAY } \\ 0.27 & \text { CALC SILTY CLAY }\end{array}$

0.30 CALC SILIY CLAY

0.48 CALC SILTY CLAY
0.22 CALC SILIY CLAY

SITE 23E: LAT 1 DEG 41 NIN S: LCNG 57 OEG 39 MIN E; OEPIF 4487 M (ANAL LI)

DEPTH SIO2

3.14
$3: 45$
$2: 81$
$3: 70$

5.640 .10

$0.15 \quad 3.30 \quad 22: 40 \quad 0: 89$

$4.56 \quad 0.10$

$\begin{array}{ll}3.42 & 0.05 \\ 3.79 & 0.10\end{array}$

3.16
2.98

$2: 16 \quad 0.10$

$\begin{array}{ll}3.01 & 0.0 \\ 4.02 & 0.1\end{array}$

$4.32 \quad 0: 09$

$\begin{array}{ll}6.58 & 0.05 \\ 5.25 & 0.10\end{array}$

$\begin{array}{lllll}5.35 & 0: 10 & 3: 42 & 7: 30 & 1.28 \\ 5: 82 & 0: 08 & 3: 79 & 13: 80 & 1: 08\end{array}$

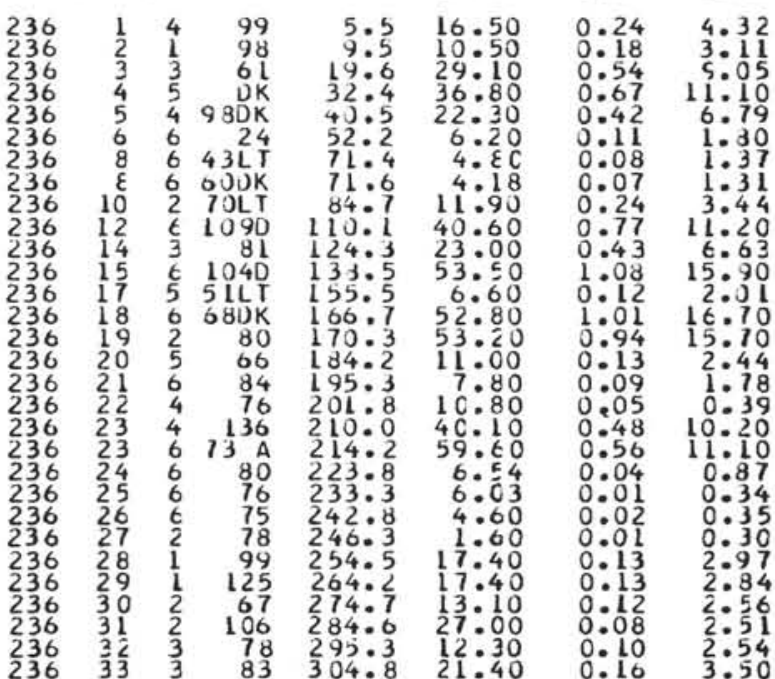

NA2O

P205 CL

LITHCLCGY

$\begin{array}{ll}2.41 & 24.00 \\ 2: 31 & 16: 00 \\ 1: 78 & 29.80\end{array}$

$\begin{array}{ll}1.02 & 47.00 \\ 0.80 & 47.00\end{array}$

0.24

$\begin{array}{lll}1.29 & 41.80 & 0.44\end{array}$

$\begin{array}{ll}3: 17 & 14.30 \\ 1.92 & 31.60\end{array}$

$0.68 \quad 45.00$

$\begin{array}{ll}3.53 & 1.55 \\ 3.58 & 1.51\end{array}$

$0.63 \quad 45.20$

0

$0.70 \quad 6.94 \quad \frac{1}{3}: 02$

$0: 33$ 4 $4: 20 \quad 00: 19$

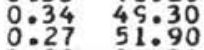

$1: 02 \quad 36.30$

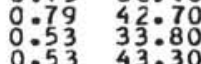

$0: 0$

0.41

0.53

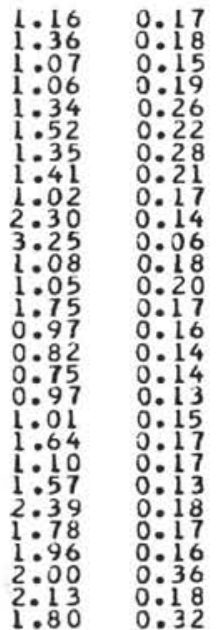

$1: 16$
$1: 29$
$1: 07$
$1: 25$
$1: 07$
$1: 09$
$0: 94$
$0: 95$
$1: 08$
$1: 33$
$1: 02$
$0: 76$
$1: 14$
$0: 78$
$0: 92$
$1: 01$
$1: 02$
$0: 91$
$0: 83$
$0: 65$
$0: 78$
$0: 85$
$0: 52$
$0: 60$
$0: 50$
$0: 64$
$0: 52$
0.46

FCRAM-NANNC OCZE

NANNO OCZE

QUANTZOOSE AANNO COZE

DCLO. CLAYEY SILT

NANNO ODZE

VOLCANIC ASH

VOLCANIC ASH

NAANC COZE

NANAC OCZE

NANNO OCZE

NANNO DOZE

NANNO OOZE

NANNO DOZE

NANNO OOZE

NANNO OOZE

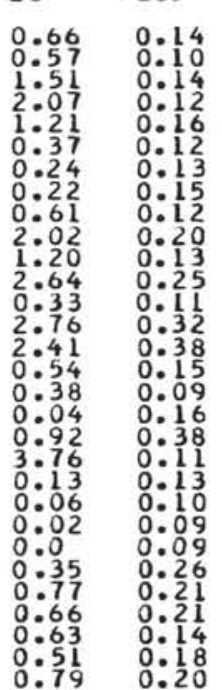

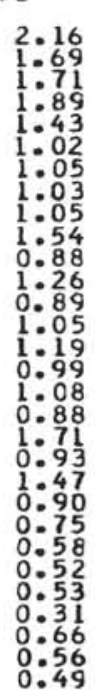

LI THCLOGY

NANNC OOZE

NANNO OOZE

NANNO OOZE

NANNO OOZE

NANNO OOLE

NNO OOZE

CLAY

CLAY

NANNO OOZE

NANNO CHALK

VCLCANIC A SH

NANNO CHALK

NANNO CHALK

NANNO CHALK

NANNO CHALK

NANNO CHALK

NANNO CHAL

AANNO CHALK
NANNO CHALK 
SITE 238: LAT I DEG 9 MIN S; LCAG 70 DEG 32 NIA E; DEPTH $2844 \mathrm{~N}$ (ANAL WALLACE) DEPIH SIO2 ILO2 AL203 FE203 MNO MGO CAO NA2O $\begin{array}{rrr}238 & 1 & 5 \\ 238 & 2 & 2 \\ 238 & 4 & 6 \\ 238 & 10 & 6 \\ 238 & 16 & 3 \\ 238 & 22 & 6 \\ 238 & 28 & 5 \\ 238 & 35 & 4 \\ 238 & 41 & 5 \\ 238 & 47 & 4 \\ 238 & 50 & 3 \\ 238 & 53 & 5\end{array}$
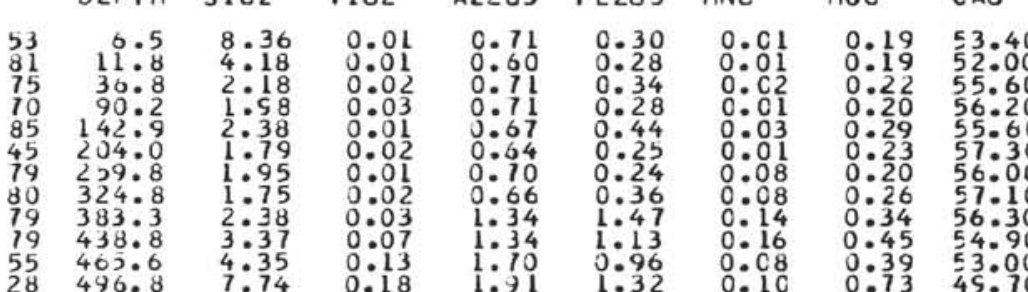

0.27

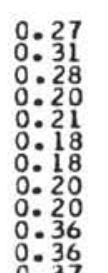

SITE 24L: LAT
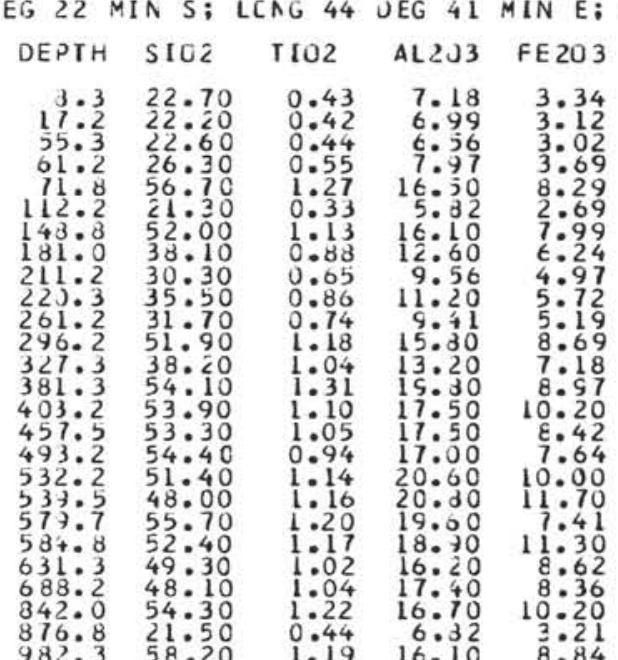

CEPTH 4054 M (ANAL LI)

ITE $250: L A T 33$ DEG 28 MIN S;LCAG 39 DEG 22 MIN E; DEPTH 5119 M (ANAL LI)

SAMPLE DEPTH SIC 2

102 AL2U3 FE2O3 MNO

MGC

$\begin{array}{rr}250 & 1 \\ 25 J & 1 \\ 250 & 2 \\ 250 & 2 \\ 250 A & 1 \\ 250 A & 1 \\ 250 A & 3 \\ 250 A & 3 \\ 250 A & 4 \\ 250 A & 4 \\ 250 A & 5 \\ 250 A & 6 \\ 250 A & 7 \\ 250 A & 8 \\ 250 A & 5 \\ 250 A & 10 \\ 250 A & 11 \\ 250 A & 12 \\ 250 A & 13 \\ 250 A & 14 \\ 250 A & 15 \\ 250 A & 16 \\ 250 A & 18\end{array}$

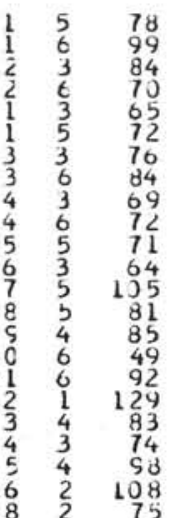

$\begin{array}{rr}0.8 & 38.80 \\ 3.5 & 40.70\end{array}$

$\begin{array}{ll}0.64 & 12.70 \\ 0.72 & 14.40\end{array}$

6.06

0.34

0.34
$0: 49$
$0: 14$
$0: 19$
$0: 02$
$0: 30$
$0: 24$
$0: 16$
$0: 30$
$0: 07$
$0: 03$
$0: 08$
$0: 17$
$0: 08$
$0: 06$
$0: 38$
$0: 05$
$0: 04$
$0: c 7$
$0: 23$
$0: 05$
0.66
0.23

\section{$\begin{array}{ll}1.87 & 14.20\end{array}$}

$\begin{array}{rr}1.98 & 12.80 \\ 1.70 & 3.35 \\ 2: 37 & 3: 23 \\ 2.42 & 0.58 \\ 2.45 & 3.37\end{array}$

$\begin{array}{ll}58: 1 & 54: 30 \\ 61: 2 & 51: 40\end{array}$

0.91 19:10

$\begin{array}{ll}0.85 & 17: 30 \\ 0.01 & 12.30\end{array}$

7.51

5.49
8.84

$0.92 \quad 19: 10$

7.83

$119: 2 \quad 44: 60$

191:1 $55.4 \mathrm{C}$

$299.8 \quad 55.20$

$414: 3 \quad 53.80$

$471: 4 \quad 55: 5$
$521: 3$
572

$607: 2 \quad 57: 10$

$636.6 \quad 32.50$

$18 .+0$

$.96 \quad 19.30$

$0.91 \quad 18.50$

$\begin{array}{ll}0.92 & 18: 40 \\ 0.92 & 18: 40\end{array}$

0.98 17:.50

8.27
7.83

8.83

8.04
9.09

$8: 11$
$8: 23$
$1: 78$
$8: 10$

$\begin{array}{lll}0.65 & 10: 30 & 8.167 \\ 1.00 & 17.20 & 9.19\end{array}$

$\begin{array}{rrr}0.66 & 2.48 & 0.77 \\ 0.23 & 3.32 & 22.40 \\ 0.06 & 0.90\end{array}$

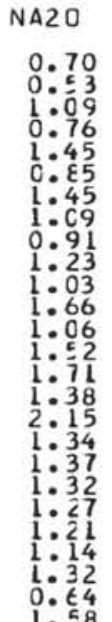

20

P205

$\mathrm{CL}$

LITHOLOGY

$\begin{array}{ll}0.11 & 0.06 \\ 0: 06 & 0: 05 \\ 0: 08 & 0: 08 \\ 0: 07 & 0: 05 \\ 0: 05 & 0: 07 \\ 0: 06 & 0: 08 \\ 0: 06 & 0: 07 \\ 0: 04 & 0: 05 \\ 0: 11 & 0: 12 \\ 0: 14 & 0: 16 \\ 0: 25 & 0: 13 \\ 0.23 & 0.14\end{array}$

1.55
1.49
1.47

FCRAM NANNC OOLE

1.51 FCRAM NANNO OOZE

1031 NANNO DOZE OOZ

0.79

NANAO OOZE

1.00

0.63

NANNO OOZE

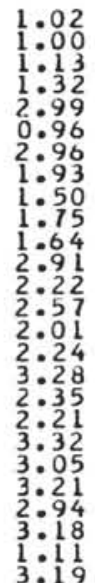

P205

0.100

0.11

0.16

$0: 17$

0.13

0.14

$0: 16$

0.16

0.19

0.16

$0: 13$

0.14
0.17

K20 P205

$205 C$

0.85
$1: 11$
1.08
$1: 09$
$1: 44$
1.39

$\begin{array}{rr}2: 45 & 3: 37 \\ 1.84 & 17.20\end{array}$

$2.51 \quad 21.67$

$2.67 \quad 0.75 \quad 1.54$

$2.68 \quad 0.72 \quad 1.50$

2.68
2.63
2.75
2.61

2.60

0.69

$\begin{array}{ll}0.60 & 1.50 \\ 2.41 & 1.39\end{array}$

2.406

$1: 5$

1.48
1.63

1.88

$2: 56 \quad 0: 15$

$2: 53 \quad 0: 18$

$\begin{array}{ll}1.87 & 0.15\end{array}$

$\begin{array}{ll}2: 24 & 0: 15 \\ 2: 74 & 0: 15\end{array}$

$\begin{array}{ll}2.93 & 0.18\end{array}$

$2.93 \quad 0.15$

$2.76 \quad 0.14$

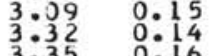

$3: 19 \quad 0: 16$

$\begin{array}{ll}1.87 & 0.10 \\ 3.35 & 0.18\end{array}$

CL

LI THOLOGY

1.77 CLAYEY NANAO OOZE

1113 CLAYEY NANAO OOZE

0.49 CLAYEY NANNO OOZ

0.99 NANNO-RICH CLAY

0.74 CLAYEY NANNO DOZE

0.71 CLAYEY NANNO ODZ

0.54 CLAYEY NANNO

0.66 CLAYEY NANNO DOZE

0.45 SILIY CLAY

0.56 CLAYSTCNE

0.37 CLAYSICNE

.36 CLAYSTCN

0.71 CALCAREOUS CLAYSTONE

1.14 CALCAREOUS CLAYSTONE
0.26 CALCAREOUS CLAYSTONE

$\mathrm{CL}$

LI THCLCGY

2.85 SILTY CLAY

2.68 SILIY CLAY

$1: 6 C$ CLAY 14 CLAYY UOZE

1.87 CLAYEY DOZE

1.19 CLAYEY OOZE

1.10 CLAY

1.15 CLAY

0.88 CLAY

1.03 CLAY

0.51 CLAY

0.90 CLAY

0.31 NANNO DOZE 
SANPLE $\mathrm{T} 102$ AL2C3 FE2O3 MN MGC CAO NA2C K 20 P205 $\mathrm{CL}$

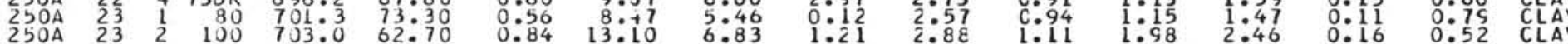
SITE 2E1: LAT 36 DEG 30 NIA S; LCNG 49 [Ej $\angle \rightarrow$ MIA E; DEPTH 3489 M(ANAL TERFANA) SAMPLE DEPTH SIC2 TIO2 AL2J3 FE2O3 MNG MG

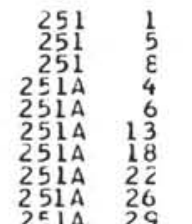

$$
\begin{array}{rrrr}
2 & 100 & 2.5 & 5: 82 \\
6 & 74 & 33: 7 & 7: 68 \\
6 & 74 & 57: 2 & 4.72 \\
4 & 70 & 111: 7 & 5: 64 \\
0 & 74 & 133: 7 & 4: 54 \\
5 & 75 & 255: 8 & 11: 80 \\
1 & 120 & 373: 7 & 8: 30 \\
3 & 75 & 414: 3 & 700 \\
2 & 120 & 451: 2 & 30: 90 \\
1 & 71 & 477.7 & 8.36
\end{array}
$$

$\begin{array}{lr}0.08 & 1: 03 \\ 0: 08 & 2: 36 \\ 0: 08 & 1: 38 \\ 0: 08 & 2: 12 \\ 0: 08 & 1: 38 \\ 0: 14 & 3: 00 \\ 0: 11 & 2: 08 \\ 0: 12 & 3: 10 \\ 0: 50 & 10: 10 \\ 0.14 & 2: 55\end{array}$

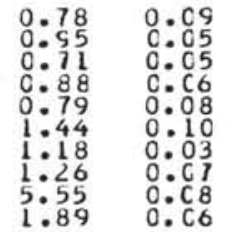

$$
\text { MGC }
$$

CAO

NA2O K

SITE 252: LAT

$$
\text { SAMPLE }
$$$$
\text { DEPTH SIOL }
$$
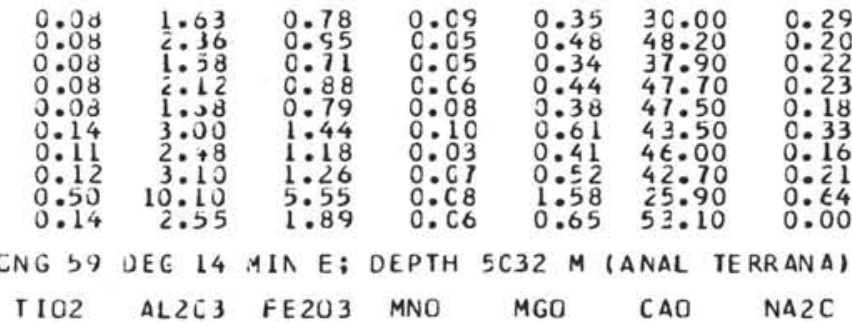

P205 CL

0.32
0.38
0.17

0.11
$0: 07$
$0: 09$
$0: 09$
$0: 10$
$0: 12$
$0: 08$
$0: 08$
$0: 18$

$1: 33$ NANAC CCZE

0.97 NANNO OOZE

0.82 NANNC OOZE

0.54 NANNC CHALK

.79 NANNC CHALK

0.85 NANNO CHALK

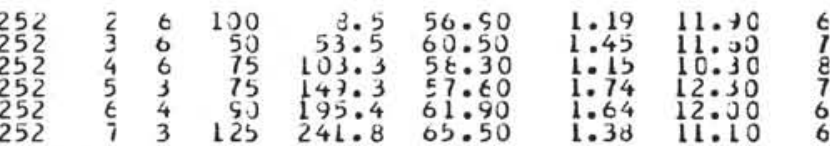

$\begin{array}{ll}6.56 & 0.1 \\ 7.58 & 0: 1 \\ 8: 03 & 0: 1 \\ 7: 61 & 0: 1 \\ 6: 99 & 0: 3 \\ 6.57 & 0.1\end{array}$

MNO $M G O$ NAO C

K20

P205 CL

L

LITHCLCGY

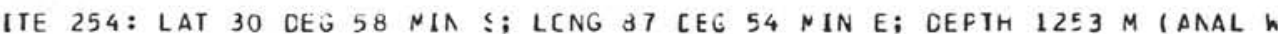
DEPIH SIO2 TIU2 AL2J3 FE2O3 MNC

$\begin{array}{lll}254 & 1 & 3 \\ 254 & 2 & 2 \\ 254 & 2 & 6 \\ 254 & 3 & 6 \\ 254 & 4 & 6 \\ 254 & 5 & 2 \\ 254 & 5 & 6 \\ 254 & 6 & 6 \\ 254 & 8 & 1 \\ 254 & 1 C & 6 \\ 254 & 12 & 4 \\ 254 & 17 & 1 \\ 254 & 18 & 6 \\ 254 & 19 & 6 \\ 254 & 15 & 6 \\ 254 & 20 & 3 \\ 254 & 20 & 6 \\ 254 & 22 & C C \\ 254 & 24 & 1 \\ 254 & 25 & 2\end{array}$

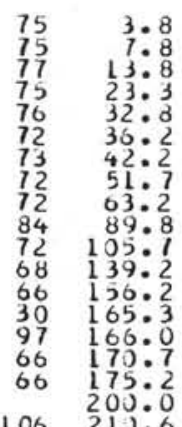

0.50

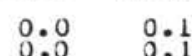

$0.10 \quad 0.03$

MNC MGO

$C \triangle O$

NA2C

2.28
2.06
1.87
1.86
1.77

0.24

2.94
4.09

RAD SILTY CLAY 2.32 RAD SILIY CLAY

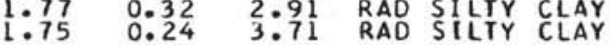

TITE 262: LAT 10 DEG 52 MIN S; LCAG 123 CEU 5L MIN E; CEFTH 23C8 M (ANAL BUDD)

\begin{tabular}{rrr}
\multicolumn{4}{c}{ SANPLE } \\
2 & 2 & 4 \\
2 & 4 & 3 \\
2 & 6 & 6 \\
2 & 8 & 0 \\
2 & 10 & 1 \\
2 & 12 & 6 \\
2 & 14 & 1 \\
2 & 16 & 6 \\
2 & 18 & 4 \\
2 & 20 & 3 \\
2 & 22 & 5 \\
2 & 24 & 6 \\
2 & 26 & 5 \\
2 & 29 & 5
\end{tabular}

$\begin{array}{llll}0.02 & 0.02 & 0.25\end{array}$

$0.50 \quad 0.0$

0.006

0.16

$0.13 \quad 0.01$

0.02

$0: 00$

$\begin{array}{lll}0.80 & 0.02 & 0.24 \\ 1.40 & 0.02 & 0.70\end{array}$

$\begin{array}{ll}0.04 & 0.00 \\ 0.04 & 0.00\end{array}$

$0.11 \quad 0.08$

$0: 1 \frac{1}{0}$

0.0
$0: 01$
0.01

0.01

0.0

$0.20 \quad 54.50$

$\begin{array}{ll}0.25 & 0.02 \\ 0.16 & 0.04\end{array}$

P205 CL

CL

LITHCLCGY

0.018050 .60

0.21156 .40

0.27

$\begin{array}{ll}0.23 & 55.80 \\ 0.43 & 54.70\end{array}$

$0.37 \quad 56.60$

$0.65 \quad 55.40$

$0.13 \quad 0.60 \quad 0.1$

$\begin{array}{ll}4.04 & 0.0 \\ 2.53 & \mathrm{C} .2\end{array}$

$\begin{array}{ll}4.73 & 2.44 \\ 4.67 & 10.20\end{array}$

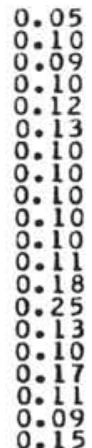

1.05

NANNO-FORAM OOLE
NANNO-FORAM OOZE

$1: 94$ NANNO-FORAM DOZE

08 NANNO-FORAM UOLE

1.04 NANNO-FORAM CCZE
1.02 NANNO-FORAM OOZE

$0: 75$

$0: 94$
$0: 86$
$1: 22$
1.01

NANNO-FORAM OOZE

NANNO-FORAM

1.01
0.93
0.67

NANNO-FORAN DOZE NANND-FORAM OOZE

.6 NANNC-FORAM $00 Z$

0.70 NANAC-FCRAM CCZE

0.58

SANDY MUCSTCNE
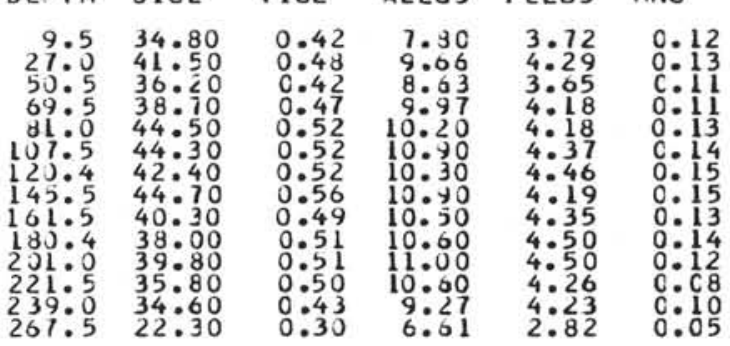

MGO

CAO

NA2C
0.80
$0: 83$
$0: 81$
$0: 82$
$0: 58$
$0: 56$
$1: 10$
$1: 14$
$0: 99$
$0: 93$
$0: 57$
$0: 91$
$0: 92$
0.74

$\mathrm{K} 2 \mathrm{O}$

P205 CL

LITHCLCGY

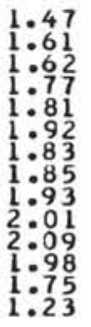

0.13
$0: 15$
$0: 16$
$0: 17$
$0: 18$
$0: 18$
$0: 16$
$0: 16$
$0: 16$
$0: 15$
$0: 13$
$0: 113$
$0: 17$
0.17

CLAYEY NANAD OOZE

$0: 96$ CALCARECUS CLO

0.64 CLAYEY OOZE DOZE

. 35 CLYEY DOZY

0.44 CLAYEY OOZE OLZ

0.54 SIL CLAYEY OOZE

0.37 CLAYEY OOZE

56 CLAYEY OOZE

0.68 CLAYEY DOZE 


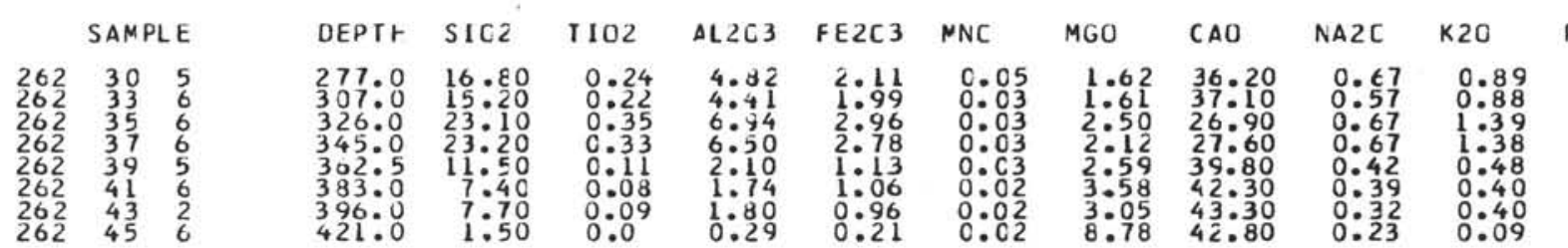

P205

$0: 19$ 0.14 0.23 $0: 20$

SITE 266 : LAT 50 DEG 24 MIN S; LONG 110 LEG 7 NIN E; DEPTH 4173 M (ANAL LI$$
\text { SAMPLE }
$$

$4 \mathrm{GO}$

\begin{abstract}
$C A O$
\end{abstract}
NA2O K2O
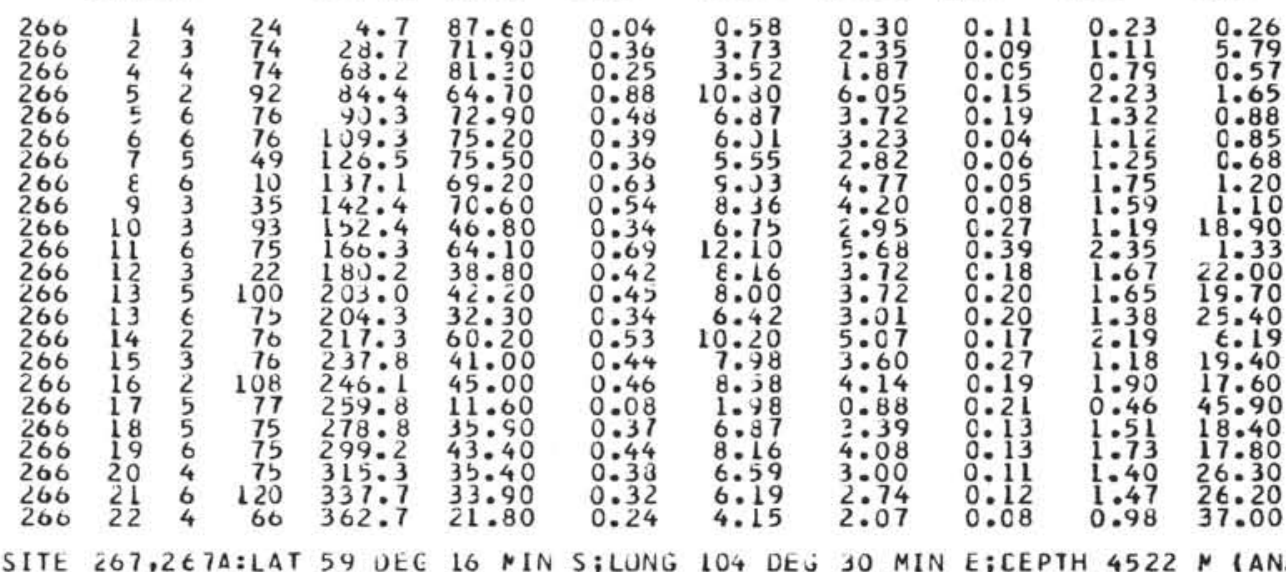

SITE 267,267 DEPIH SIU2

S:LUNC

0.12

0.98

37.20

$\begin{array}{rr}2674 & 1 \\ 267 & 3 \\ 267 & 4 \\ 267 & 5\end{array}$

$\begin{array}{lrlll}75 & 9: 3 & 68.70 & 0.73 & 5.00 \\ 75 & 96: 3 & 61: 50 & 0: 75 & 11.00\end{array}$

$\begin{array}{lll}75 & 135.8 & 34.60 \\ 46 & 167.5 & 14.00\end{array}$

0.38

$2: 71$

5.36
$5: 99$
$6: 35$

2.04

0.06

0.19
0.29
$0: 18$
0.39

$M G C$

$C A D$

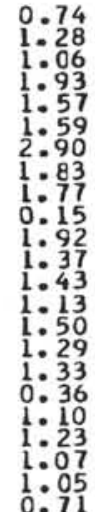

SITt $267 \mathrm{~B}$;

LAT $5 \rightarrow$ ULG 16 NIN S:LUNG

SAMPLE DEPTH SIC2 TIO2 AL2O3 FE2C3 MNO MGO CAO NA2C

$\begin{array}{ll}2670 & 4 \\ 267 & 5 \\ 2675 & 5 \\ 2675 & 7 \\ 2670 & 8 \\ 2675 & 9\end{array}$

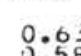

$\triangle 1203$
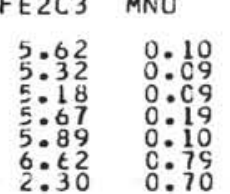

$0: 109$
$0: 09$
$0: 19$
$0: 10$
$0: 79$
0.70

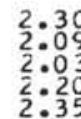

$\begin{array}{rr}2: 30 & 0.93 \\ 2: 09 & 1: 06 \\ 2: 03 & 0: 96 \\ 2: 20 & 0: 90 \\ 2: 35 & 1: 07 \\ 3 & 13 \\ 0.87 & 48.24\end{array}$

L MIN E; CEPTH 1232 MIANAL hALLACE

SITE 277: LAT

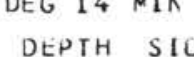

$\begin{array}{lll}277 & 1 & \\ 277 & 1 & 2 \\ 277 & 1 & 3 \\ 277 & 4 & 6 \\ 277 & 10 & 6 \\ 277 & 23 & 3 \\ 277 & 29 & 2 \\ 277 & 35 & \\ 277 & 41 & \\ 277 & 44 & \\ 277 & 46 & \end{array}$

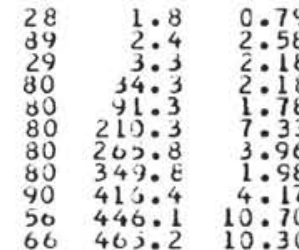

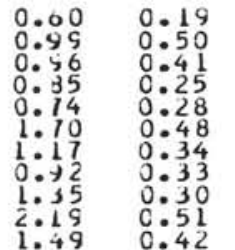

0.01
0.02
0.01
$0: 03$
$0: 03$
0.03
$0: 03$
0.08
0.04
0.08
0.02
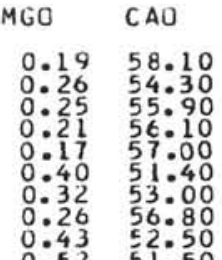

54.30
55.90

$.21 \quad 56: 10$

$.40 \quad 51.40$

$.26 \quad 56.80$

$0.521 \quad 51: 50$
CL

0.51
0.58
0.53

L ITHCLCGY

CLAYEY OOZE

NANNO-FORAM OOZE

0.45 NANAYEY CALC OOZE

0.45 CALCARECUS OOZE
0.36 DCLOMITIC CCZE

P205 CL

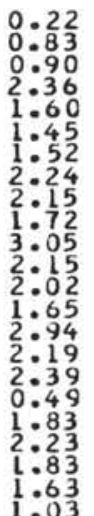

$0: 05$
$0: 10$
$0: 07$
$0: 17$
$0: 10$
$0: 09$
$0: 08$
$0: 13$
$0: 16$
$0: 10$
$0: 18$
$0: 14$
$0: 16$
$0: 12$
$0: 19$
$0: 18$
$0: 20$
$0: 08$
$0: 14$
$0: 16$
$0: 17$
$0: 12$
0.12

$\mathrm{CL}$

LITHCLCGY

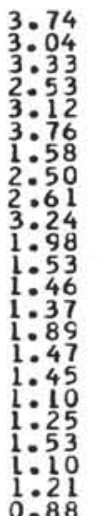

CIATCM OOZE

DIATCM DOZE

DIATCM DOZE

DIA TCMACEOLS CLAY

CLAY-CIA NAMNO OOZE

SilífeOUS Clay

NANNO DOZE

SILICEOUS CLAY
CLAYEY NANNC DOZE
CALCARECUS CLAY

NANNO OOZE

CLAYEY CHALK

NANNO OOZE

NANNC CLAYSTCNE

$\times 20 \quad P 205 \quad C L$

LITHOLOEY

2.31
3.00
3.73

0.132 .34

CLAY IILEOUS CLAY

$\begin{array}{ll}0.15 & 1.25 \\ 0.16 & 1.22\end{array}$

NANNO OOZE , CHALK

$\mathrm{CL}$

LITHCLCGY

1.73
1.60

1.61 SILICECLS CLAY

1.22 SILICEOUS CLAY
1.12 SILICEOUS CLAY

0.08

0.20

$\begin{array}{ll}0.42 & 0.06 \\ 0: 29 & 0: 13 \\ 0.23 & 0: 10\end{array}$

$0.20 \quad 0: 05$

$0: 24 \quad 0: 14$

$\begin{array}{ll}0.25 & 0: 11 \\ 0.44 & 0: 32\end{array}$
LITHCLCGY

$\begin{array}{lll}0.18 & 1.35 & \text { CALC. OOZE } \\ 0.06 & 1.04 & \text { CALC: OOZE }\end{array}$

0.14 1:03 CALC: OOZE

0.23 0.82 CALC: OOZE

0.11 0.75 CALC. OOZE

$\begin{array}{lll}0.13 & 0.67 & \text { NANNO CHALK } \\ 0.14 & 0: 38 & \text { GLAUC. CHALK } \\ 0.15 & 0.38 & \text { NANNO CHALK }\end{array}$ 
SITE 278; LAT 50 UEG 34 MIN S; LONG 16J CEU 4 NIN E; DEPTH 37 IC M (ANAL hALLACE) DEPTH SIC2 TIO2 AL2U3 FEZC3 MNO MG

$\begin{array}{rr}278 & 1 \\ 278 A & 2 \\ 278 & 2 \\ 278 & 3 \\ 278 & 3 \\ 278 & 4 \\ 278 & 5 \\ 278 & 6 \\ 278 & 7 \\ 278 & 8 \\ 278 & 8 \\ 278 & 9 \\ 278 & 10 \\ 278 & 11 \\ 278 & 12 \\ 278 & 13 \\ 278 & 14 \\ 278 & 15 \\ 278 & 16 \\ 278 & 18 \\ 278 & 20 \\ 278 & 22 \\ 278 & 23 \\ 278 & 24 \\ 278 & 27 \\ 278 & 29\end{array}$
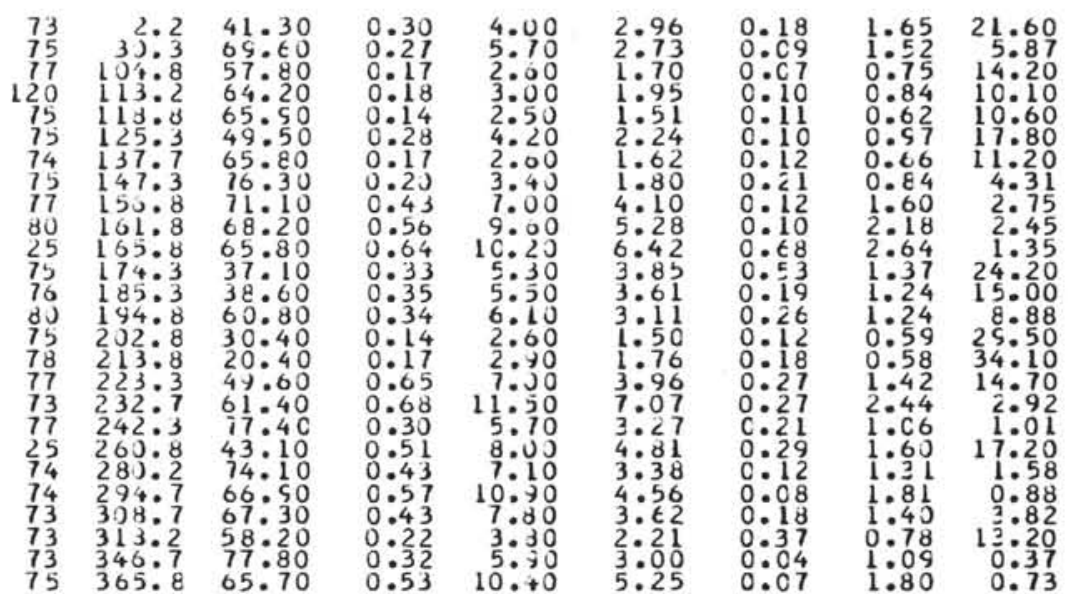

NA2C
1.41
$1: 68$
$0: 60$
$0: 92$
$0: 87$
$1: 16$
$0: 94$
$1: 69$
$2: 46$
$2: 54$
$2: 47$
$1: 03$
$1: 42$
$0: 61$
$0: 56$
$1: 56$
$2: 35$
$1: 28$
$1: 30$
$1: 66$
$1: 33$
$1: 0$
1.5
1.74

$k 20$
0.41
$0: 78$
$0: 44$
$0: 54$
$0: 46$
$0: 75$
$0: 44$
$0: 63$
$1: 26$
$1: 56$
$1: 68$
$1: 23$
$1: 23$
$1: 27$
$0: 56$
$0: 57$
$1: 54$
$2: 03$
$1: 27$
$1: 31$
$1: 55$
$2: 27$
$1: 59$
$0: 82$
$1: 36$
2.41

SITE 283; LAT

DEPTH SIC2

TIO2 154 DES 17 MIN E; DEP

\title{
DEPTH 4766 M(ANAL MALLACE)
}

SAMPLE
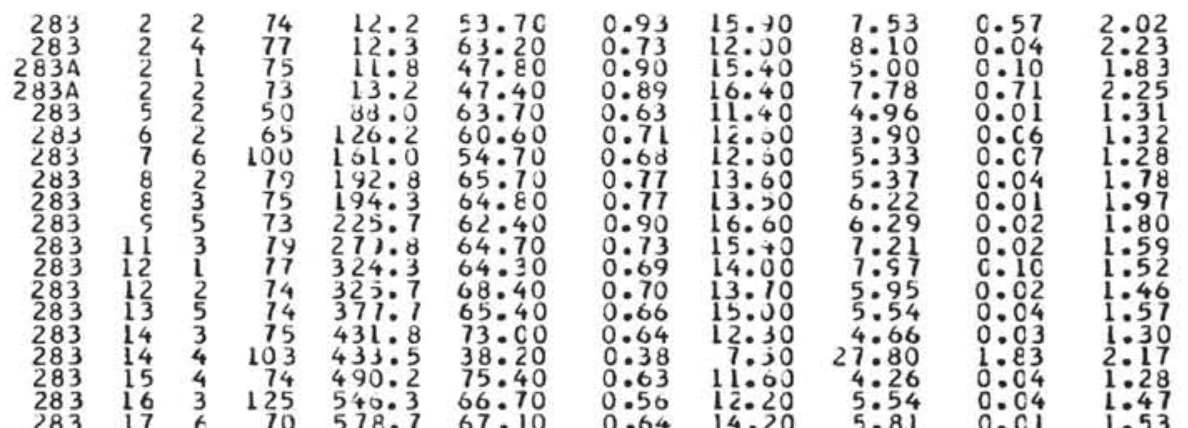

CAO

NA2C K 20

$20 \quad P 205 \quad$

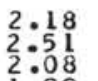

0.47 $\begin{array}{ll}1.02 & 1: 31 \\ 0.54 & 1: 61 \\ 3: 62 & 1: 02 \\ 0.57 & 1: 37 \\ 0.62 & 1: 57\end{array}$

4.74
6.87 $130: 063: 70$

$\begin{array}{ll}151: 0 & 54: 70 \\ 192: 8 & 65.70 \\ 194: 3 & 64.80\end{array}$

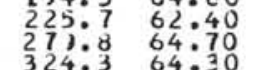

$\begin{array}{ll}324: 3 & 64: 30 \\ 327: 7 & 68: 40 \\ 377: 7 & 65: 40\end{array}$

$\begin{array}{ll}431.8 & 73.00 \\ 433: 5 & 38: 20\end{array}$

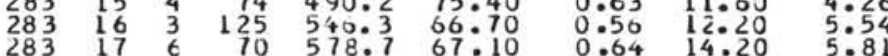

0.01

$\begin{array}{lll}.46 & 0: 75 \\ .47 & 0.40 & 0.95 \\ 53 & 0.38 & 0.81\end{array}$

SITE 289: LAT O DEG $30 \mathrm{NIA} \mathrm{S;} \mathrm{LCNG} 158$ CEG 31 MIN E; DEPIH 2224 M(ANAL WALLACE)

SAMPLE

DEPTH SIC2 TIOZ

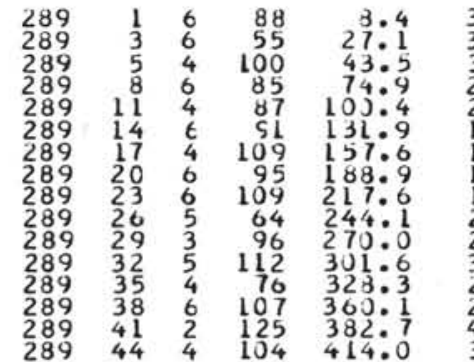

3.90
$3: 40$
$3: 10$
$2: 70$
$2: 40$
$1: 50$
$1: 30$
$1: 30$
$1: 60$
$2: 00$
2.60
$3: 50$
$2: 30$
$2: 50$
$4: 70$
$3: 50$

$\begin{array}{lll}0.20 & 1.16 & 0.38 \\ 0.04 & 0.95 & 0.44 \\ 0.04 & 0.90 & 0.24\end{array}$

MNO MGO

\begin{abstract}
GO
CAO
\end{abstract}

$\mathrm{NA} 2 \mathrm{O}$

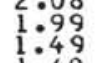

1.40 0.1

0.09

1.64
1.60
1.89

1.63
1.64
1.70

1.890 .0

$\begin{array}{ll}1.78 & 0.06 \\ 1.07 & 0.23\end{array}$

$1: 70$
1.85
1.86

0.06

K20 P205 CL

0.09

0.27

$\begin{array}{lll}0.03 & 0.02 & 1.09 \\ 0.02 & 0.49 & 0 . t 3\end{array}$

0.05
$0: 03$
0.05
$0: 00$
$0: 03$
0.100
0.02

0.02

0.2900 .40

0.02

$0.0 \quad 0.36$

$\begin{array}{lll}102 & 0.20 & 53.80 \\ 0.22 & 54.00\end{array}$

$0.22 \quad 54.00$

$0: 0$

$\begin{array}{lll}0: 02 & 0: 37 & 0: 38 \\ 0.01 & 0.26 & 0.32\end{array}$

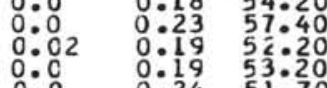

$\begin{array}{lll}0: 07 & 0.29 & 0.45 \\ 0: 02 & 0: 41 & 0: 24 \\ 0.01 & 0.31 & 0.45\end{array}$

$\begin{array}{lll}0.0 & 0.24 & 51: 70 \\ 0: 0 & 0: 24 & 51: 70 \\ 0.0 & 0.23 & 53.440\end{array}$

0.16

$0.71 \quad 0.0$

0.17

010.06

$0.21 \quad 0.05$

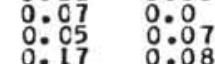

$0: 07$
$0: 07$
$0: 09$
$0: 012$
0009
$0: 07$
$0: 07$
$0: 07$
$00: 06$
$0: 07$
$0: 06$
$0: 07$
$0: 06$
$0: 09$
0.06

CL

$\mathrm{CL}$

3.33 CALC.RAC.CIAIOM COZE
2.51 CALC.RAD.CIATCM DOZE

$3: 20$ SILICECUS CCZE

$2: 52$ SILICECUS OCZE

$2: 46$ SILICEOUS OOZE

2.65

$2.0 \epsilon$

$2: 12$

2.36

$1: 68$
$1: 53$
1.87

1.87
2.85

1.71

1:.93

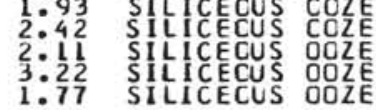

SILICECUS OCZE

SIL:NANNO: OULE

IL.NANNU: OOZE

S.NANAC:

L.NANNO: COZE

ILICECLS COZE

CALC. NANNC. ODZZE

$\mathrm{CL}$

3.75 SILIY ZEOLITIC CLAY

3.36 SILIY ZECIIIIC CLAY

4.63 SILIY LEOLITIC CLAY
2.66 SILICEOUS COZE

$2: 20$ CALC. SILICECUS CCZE

$1: 78$ SILTY SILICEOUS CLAY

0.95 SILYY CLAY

0.86 SILTY CLAY

0.75 SILTY CLAYSIONE

0.39 SILTY CLAYSTONE

0.26 SILTY CLAYSICNE

0.42

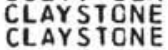

1.49
$1: 41$
$1: 26$
$1: 35$
$1: 26$
$0: 11$
$0: 47$
$1: 26$
$1: 04$
$1: 06$
$1: 06$
$1: 10$
$0: 99$
$1: 26$
0.82

LITHCLOGY

1.49 NANNC-FORAN OOZE
1.41
NANNO-FORAM OOZE

NANNC-FORAM OOZ

NANNO-FORAM OOZE

NANNO-FORAM OCZE

NANNO-FORAM OUZE

NAN.-FOR. COZE/CHALK

NAN:- FOR: CCZE/CHALK

NAN:-FOR: OOZE/CHALK
NAN:-FOR: COZE/CHALK

NAN:-FOR: OOZE/CHALK 
TABLE 3 - Continued

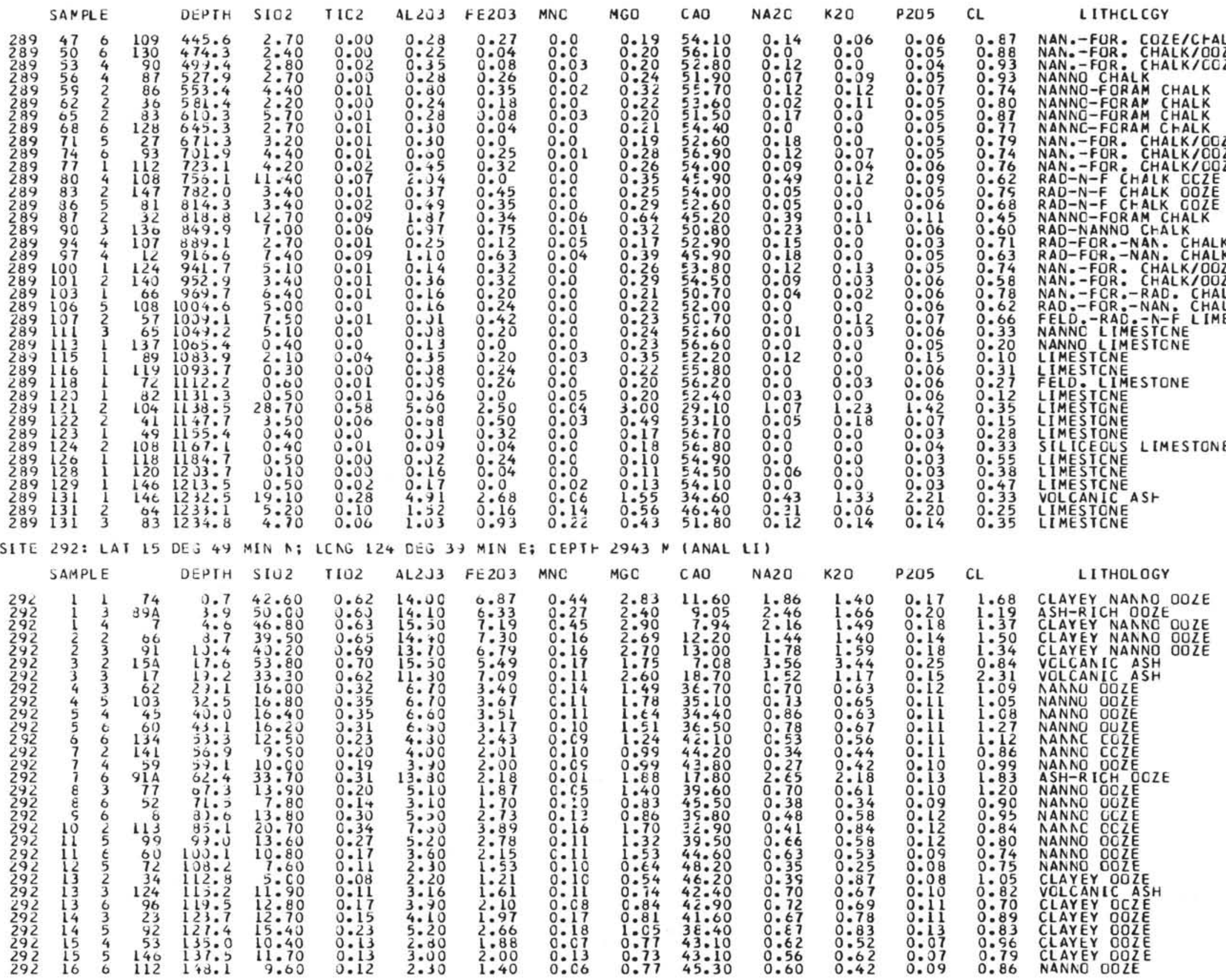


SAMPLE

DEगTH 5102 TIC

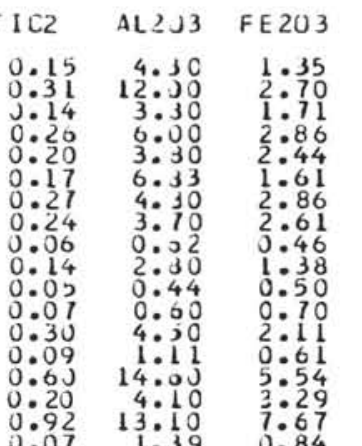

$\begin{array}{ll}1: 35 & 0: 06 \\ 2: 70 & 0: 06 \\ 1: 71 & 0: 05 \\ 2: 86 & 0: 09 \\ 2: 44 & 0: 10 \\ 1: 61 & 0: 08 \\ 2: 86 & 0: 09 \\ 2: 61 & 0: 06 \\ 0: 46 & 0: 05 \\ 1: 38 & 0: 08 \\ 0: 50 & 0: 04 \\ 0: 70 & 0: 05 \\ 2: 11 & 0: 09 \\ 0: 61 & 0: 07 \\ 5: 54 & 0: 15 \\ 3: 29 & 0: 10 \\ 7.67 & 0: 19 \\ 0.84 & 0: 07 \\ 0.40 & 0: 10\end{array}$

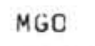

$C A O$

NA2O

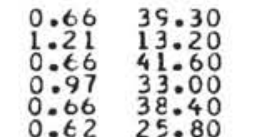

$0.06 \quad 38.40$

$\begin{array}{ll}1.24 & 36: 70 \\ 0.99 & 41.10 \\ 0.32 & 5 \mathrm{c}: 60\end{array}$

$\begin{array}{ll}0.50 & 40.10 \\ 0.30 & 50: 40 \\ 0.45 & 51: 30\end{array}$

$0.45 \quad 51.30$

$0.48 \quad 49.50 \quad 2.51$

$\begin{array}{ll}0.77 & 1.82 \\ 1.29 & 33.00 \\ 1.25 & 4.15\end{array}$

$\begin{array}{ll}1.25 & 4.15 \\ 0.64 & 40: 00 \\ 4.43 & 11: 00\end{array}$

4.21

$\begin{array}{llllll}292 & 37 & 5 & 70 & 336: 3 & 23: 10 \\ 292 & 37 & 3 & 118 & 341: 0 & 56: 50 \\ 292 & 38 & 1 & 119 & 343: 5 & 11: 30\end{array}$

$\begin{array}{lll}0.07 & 1.39 \\ 2.81 & 13.20 & 0.8\end{array}$

SITE 296: LAT 29 DEG $20 \mathrm{MIN} \mathrm{N}$; LCNG 133 CEJ $32 \mathrm{MIN}$ E; CEFTH $2958 \mathrm{~N}$ (ANAL. LII

DEPTH SIUZ
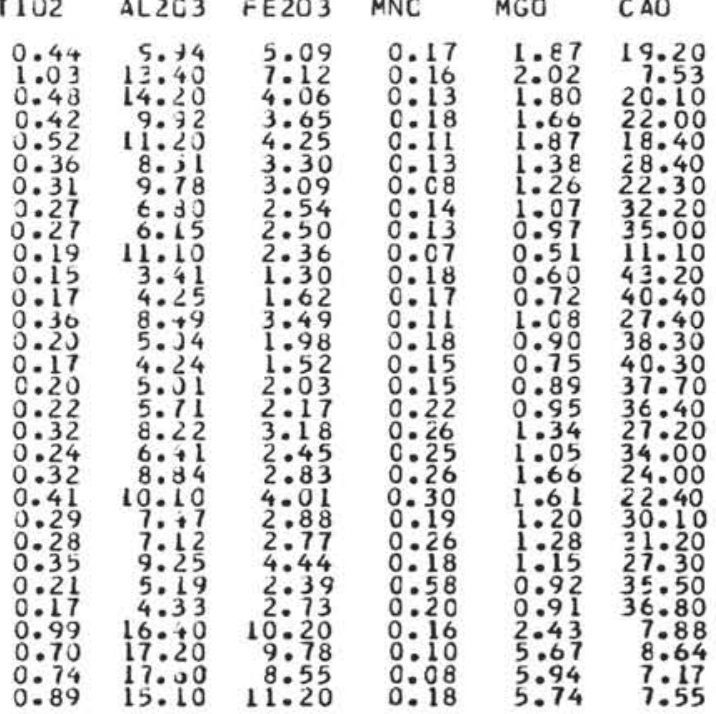

$\mathrm{NA2C}$

$K 20$
0.90
$2: 96$
$0: 37$
0.68
0.44
$1: 39$
$0: 47$
$0: 32$
$0: 09$
0.48
$0: 08$
0.07
0.68
0.11
$3: 95$
0.79
2.71
0.18
0.98

P205

$\mathrm{CL}$

LITHCLCGY
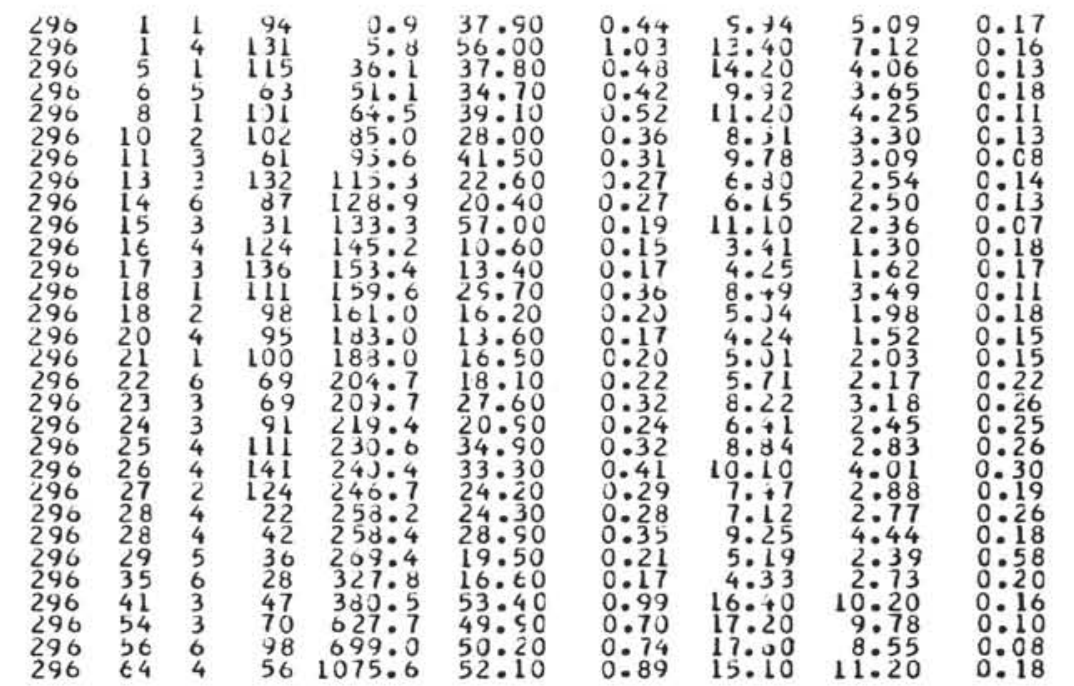

$\begin{array}{ll}2.02 & 7.53 \\ 1.80 & 20.10 \\ 1.60 & 22.00 \\ 1.87 & 18.40\end{array}$

$\begin{array}{ll}1.38 & 28.40 \\ 1.26 & 22.30\end{array}$

$\begin{array}{lll}1.07 & 32.20 \\ 0.97 & 35.00\end{array}$

$0.512 \quad 11.10$

0.72

$\begin{array}{ll}1.08 & 27.40 \\ 0.90 & 38.30\end{array}$

$0.75 \quad 40.30 \quad 0.40$

$\begin{array}{lll}0.89 & 37.70 & 0.52 \\ 0.95 & 36.40 & 0.61\end{array}$

$1.0534 .00 \quad 0.66$

1.6624 .00

$\begin{array}{lll}1.61 & 22.40 & 1: 09 \\ 1.20 & 30: 10 & 0.77\end{array}$

$1.28 \quad \frac{2}{2} 7.20$

$0.92 \quad 35.50 \quad 0.68$

$2.43 \quad 7.88 \quad 2: 91$

$\begin{array}{lll}5: 67 & 8: 64 & 2: 11 \\ 5: 94 & 7: 17 & 2: 27 \\ 5.74 & 7.55 & 1: 14\end{array}$

SITE 297: LAT 30 DEG 52 MIN A: LCNG 134 CEG 10 MIA E; DEFTH 4480 M(ANAL hALLACE)

DEPIH SIO2 TIO2

AL2O3 FE2O3 MNO MGO

CAO

NA2C K2O

$\begin{array}{rrrrrr}297 & 3 & 2 & 48 & 22.0 & 60.80 \\ 297 & 4 & 5 & 78 & 45: 8 & 53: 60 \\ 297 & 5 & 6 & 64 & 65: 1 & 46: 20 \\ 297 & 6 & 6 & 83 & 95: 3 & 60: 20 \\ 297 & 8 & 6 & 48 & 113: 5 & 60: 00 \\ 297 & 10 & 6 & 83 & 161: 3 & 60.20 \\ 297 & 11 & 3 & 83 & 201: 3 & 60: 20 \\ 297 & 12 & 2 & 63 & 250: 1 & 59: 00 \\ 297 & 12 & 3 & 111 & 252: 1 & 67.40 \\ 297 & 13 & 1 & 139 & 269: 9 & 59.50 \\ 297 & 15 & 3 & 56 & 334: 1 & 58: 90 \\ 297 & 17 & 2 & 85 & 397: 9 & 80040 \\ 297 & 18 & 2 & 34 & 430: 3 & 79: 30 \\ 297 & 19 & 1 & 99 & 467.5 & 64.50\end{array}$

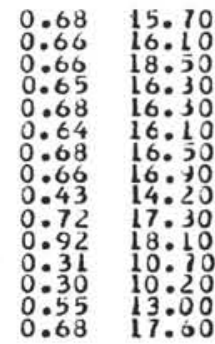

5.75
$6: 76$
$5: 20$
$5: 23$
$5: 98$
$5: 78$
$5: 57$
$6: 01$
$3: 07$
$5: 93$
$6: 19$
$2: 27$
$2: 28$
$5: 43$
$6: 36$
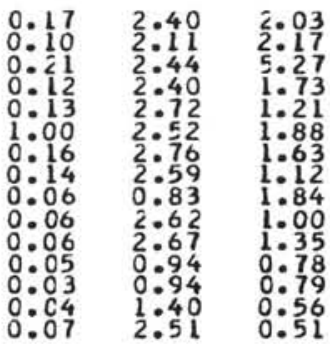

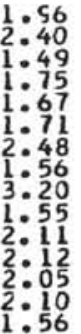
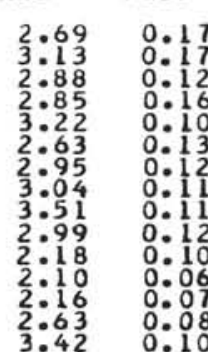

$2: 06$
$1: 3$
$1: 8$
$1: 3$
$0: 5$
$1:$
0
0
0
$0:$
0
$0:$

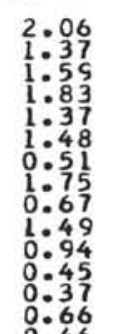

0.46 CLAYSTICNE 


\begin{tabular}{|c|c|c|c|c|c|c|c|c|c|c|c|c|c|c|}
\hline SAMPLE & & DEPTH & SIC2 & r102 & AL2J3 & FE203 & MNC & MGC & CAO & NA2 C & K20 & P205 & CL & LITHOLCGY \\
\hline $\begin{array}{lll}7 & 21 & 3 \\
7 & 22 & 4 \\
7 & 23 & 2 \\
7 & 24 & 2 \\
7 & 25 & 2 \\
7 & 25 & 2 \\
7 & 25 & 6 \\
7 & 27 & 1\end{array}$ & $\begin{array}{r}120 \\
80 \\
71 \\
68 \\
131 \\
134 \\
124 \\
125\end{array}$ & $\begin{array}{l}524.7 \\
553: 3 \\
592: 2 \\
620: 7 \\
649: 8 \\
649: 8 \\
655: 7 \\
676: 8\end{array}$ & $\begin{array}{l}60.60 \\
67: 40 \\
64: 50 \\
61: 00 \\
69: 40 \\
68: 00 \\
69: 20 \\
65.50\end{array}$ & $\begin{array}{l}0.68 \\
0: 64 \\
0: 73 \\
0: 63 \\
0: 28 \\
0: 30 \\
0: 29 \\
0.70\end{array}$ & $\begin{array}{l}17: 50 \\
15: 30 \\
17: 30 \\
15: 30 \\
13: 60 \\
13: 100 \\
16: 100\end{array}$ & $\begin{array}{l}6: 73 \\
4: 35 \\
5: 20 \\
5: 64 \\
33: 46 \\
3: 51 \\
2: 56 \\
4: 84\end{array}$ & $\begin{array}{l}0: 30 \\
c: 03 \\
0: 01 \\
0: 10 \\
0: 02 \\
0: 05 \\
0: 04 \\
0: 11\end{array}$ & $\begin{array}{l}2: 53 \\
1: 91 \\
2: 11 \\
2: 35 \\
1: 14 \\
1: 45 \\
0: 75 \\
2: 27\end{array}$ & $\begin{array}{l}0.62 \\
0: 69 \\
0: 55 \\
1: 36 \\
1: 14 \\
0: 75 \\
0: 82 \\
0.70\end{array}$ & $\begin{array}{l}1: 57 \\
1: 96 \\
1: 77 \\
1: 55 \\
2: 39 \\
2: 30 \\
2: 89 \\
1: 67\end{array}$ & $\begin{array}{l}3.50 \\
2: 87 \\
3: 46 \\
2: 97 \\
3: 97 \\
3: 63 \\
4: 09 \\
3: 24\end{array}$ & $\begin{array}{l}0: 11 \\
0: 10 \\
0: 10 \\
0: 10 \\
0: 31 \\
0: 10 \\
0: 26 \\
0: 1\end{array}$ & $\begin{array}{l}0.50 \\
0: 42 \\
0: 37 \\
0: 71 \\
0: 61 \\
0: 73 \\
0: 64 \\
0.31\end{array}$ & $\begin{array}{l}\text { CLAYSTCAE } \\
\text { SILTY CLAY } \\
\text { CLAYSTCNE } \\
\text { CLAYSTCNE } \\
\text { ASH } \\
\text { ASH } \\
\text { VCLCANIC ASH } \\
\text { CLAYSTCNE }\end{array}$ \\
\hline
\end{tabular}

SITE 305: LAT 32 DEG O NIA A; LCAG 157 CEG 51 MIN E; DEPTH 2921 M(ANAL. hALLACE)

SAMPLE DEPTH SIOZ
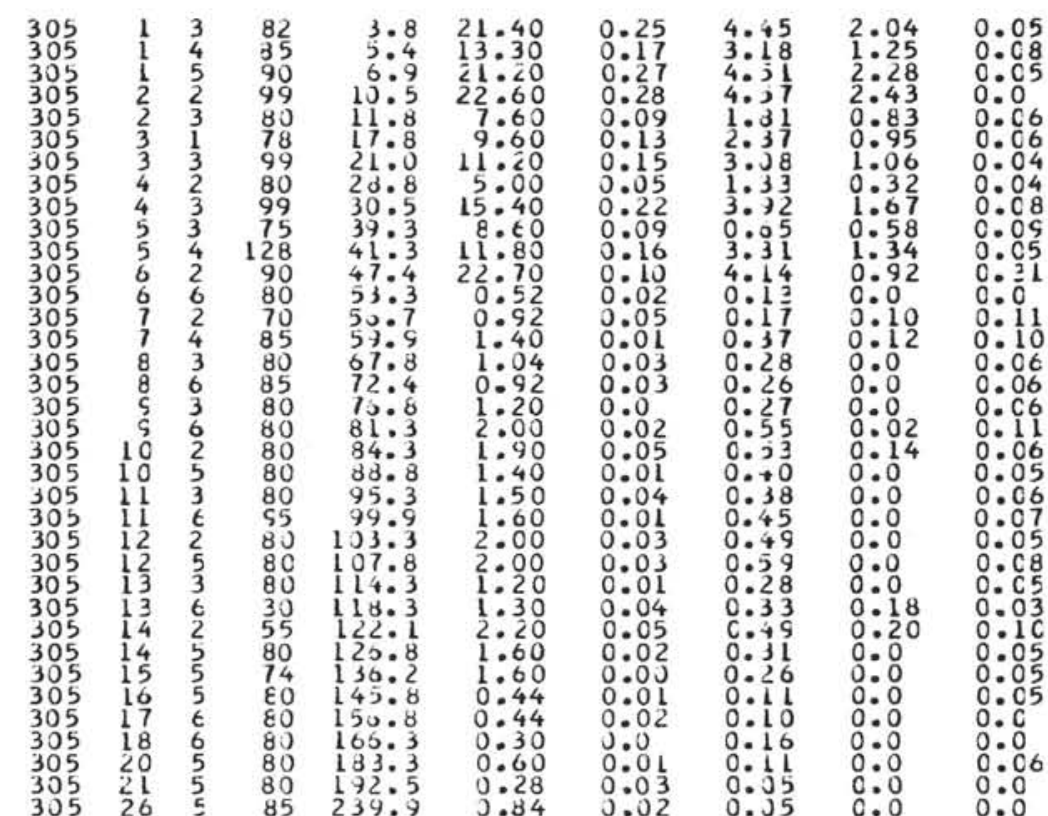

MGO

CAO

NA2C

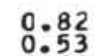

K20

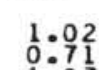

$\begin{array}{rr}0.68 & 41.10 \\ 1.08 & 33.60 \\ 1.07 & 32.40\end{array}$

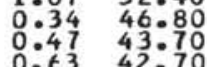

$\begin{array}{ll}0: 29 & 42: 70 \\ 0: 83 & 40: 60\end{array}$

0.83

0.69
0.44
05

$0: 0952: 60$

0.07 5. $53: 70$

$0: 14 \quad 52: 50$

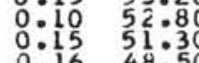

$0.16 \quad 48: 50$

$\begin{array}{ll}0.27 & 51: 60 \\ 0.26 & 52: 10\end{array}$

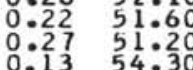

$0.16 \quad 53: 10$

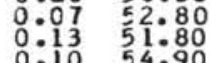

0.15 53:30

$0.12 \quad 52: 60$

$0.12 \quad 54: 50$

SITE 310: LAT 30 DEO 52 NIN NiLCNG 170 CEG 54 NIN E; DEPIH 3524 MIANAL. WALLACE

SAMPLE DEPIT SICŹ TIU2 ALZO3 FE2O3 MNO

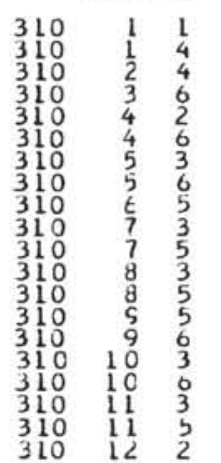

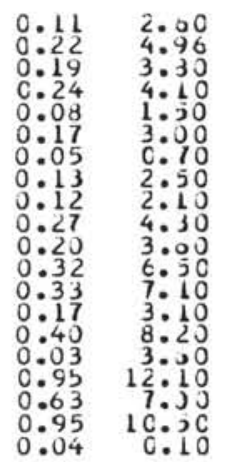

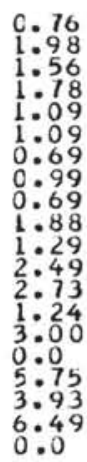

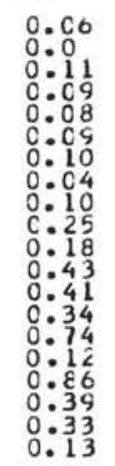

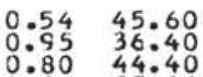

$\begin{array}{ll}0.80 & 44.40 \\ 1.10 & 37.10\end{array}$

$0.71 \quad 45: 70$

$0.04 \quad 43.60$

$1: 14 \quad 35: 60$

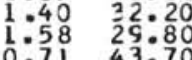

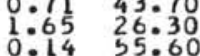

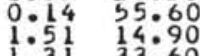
$\begin{array}{ll}1: 66 & 21: 90 \\ 0 & 15\end{array}$
$0.82 \quad 1: 3$

$0: 41$

$0.24 \quad 0: 35$

0.49

$0: 0$

$0: 10$

0.05

0.04 0.0 $0: 14$ 0.14 0.10 $0: 01$ $0: 10$ $0: 01$

$0: 23$

$0: 27$
$0: 64$
$0: 35$
$0: 49$
$0: 30$
$0: 45$
$0: 13$
$0: 29$
$0: 27$
$0: 59$
$0: 47$
$0: 71$
$1: 14$
$0: 02$
$1: 04$
$0: 03$
$2: 06$
$0: 57$
$0: 94$
$0: 04$

P205

0.08

$\mathrm{CL}$

1.54 SIL: FOR.-NAN. COLE 1:38 SIL: FOR:-NAN: OCLE 1:36 SII: FRR: NAA: DOZE 1.41 SIL: FOR.-NAN: 1:33 SIL: FOR:-NAN: OOZE $1: 26$ SIL: FOR :-NAN: DOZE $1: 29$ CLAYEY NANAC CCZE 09 AANNU OOZE 0.97 NANNC CCZE 0.86 NANAO DOZE
$1: 02$ NANNO OOZE $0: 03$ NANNO OOZE 0.82 NANNO OOZE 82 NANNO OCZE
$0: 86$ NANNO COZE NANNO OOZE 0.82 NANNO DOZE $0: 82$ NANAO CCZE $0: 83$ NANNC GOZE 0.86 NANAO COZE 10 FCRAM-NANNO CHALK 81 NANNO OOZE
0.80 NANNO OCZE $0: 77$ FORAM-NANNC CHALK

P205 CL $\begin{array}{ll}0.68 & 0.05 \\ 1: 14 & 0.09\end{array}$ $\mathrm{CL}$ LITHOLCGY

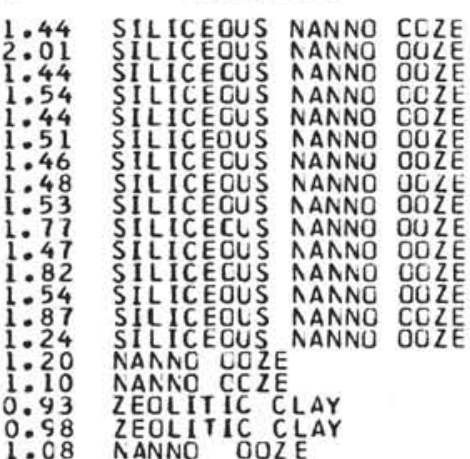

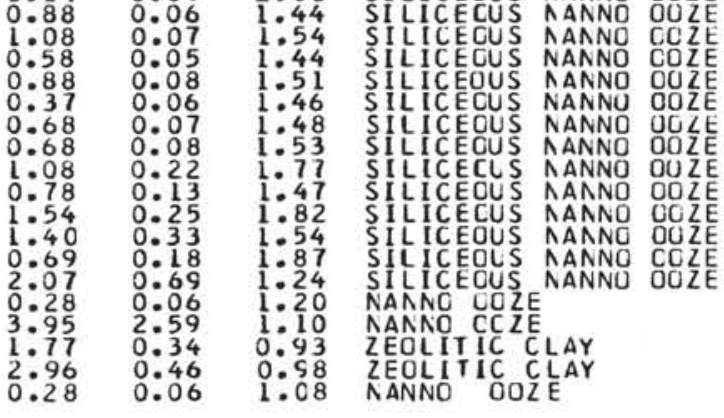




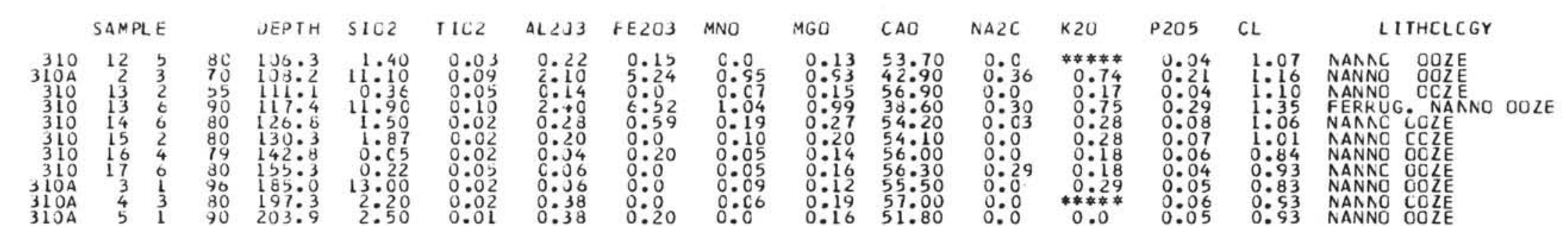

SITE 317; LAT 11 DEG J NIN S; LCAG 162 CEG IO NIN hiDEPTH 2622 M (ANAL. WALLACE) SAMPLE DEPTH SICZ TIO2 ALZJ3 FE2O3 MNC MGO CAO NA2C K

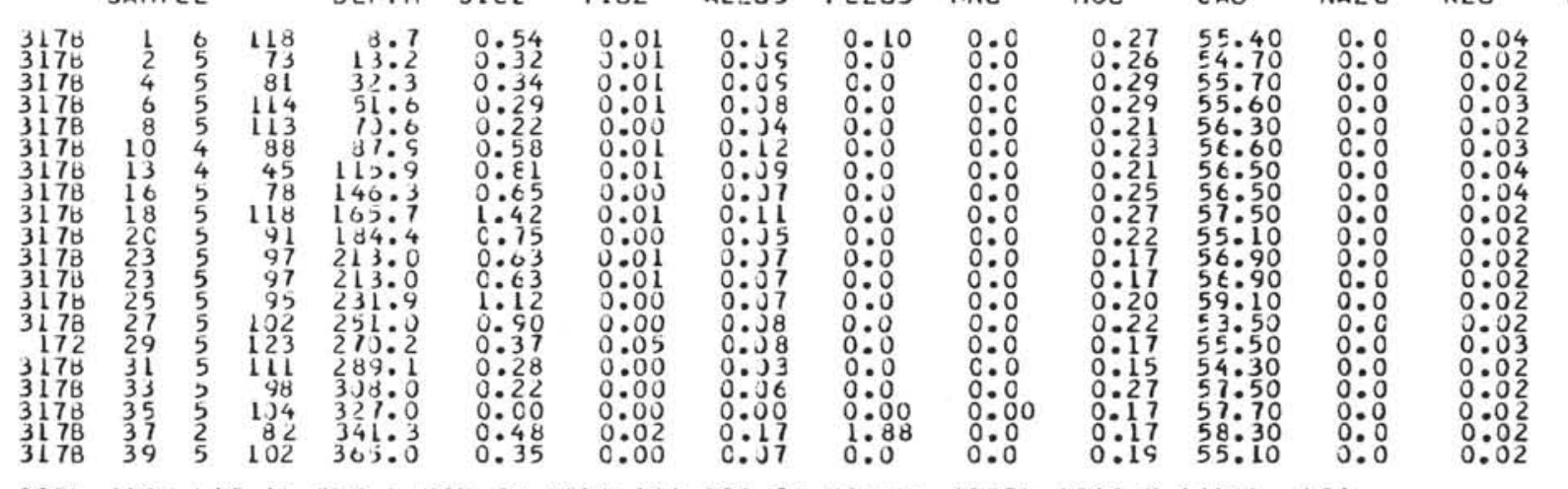

P205 CL

LITHCLOGY

SITE 3LS: LAT L3 ULG L MIN S; LCNG $10 L$ CEG 32 NIN W; CEPTH 4290 M (ANAL. LI) SAMPLE TIU2 AL2U3 FEZU3 MNO MGO CAO NA2O

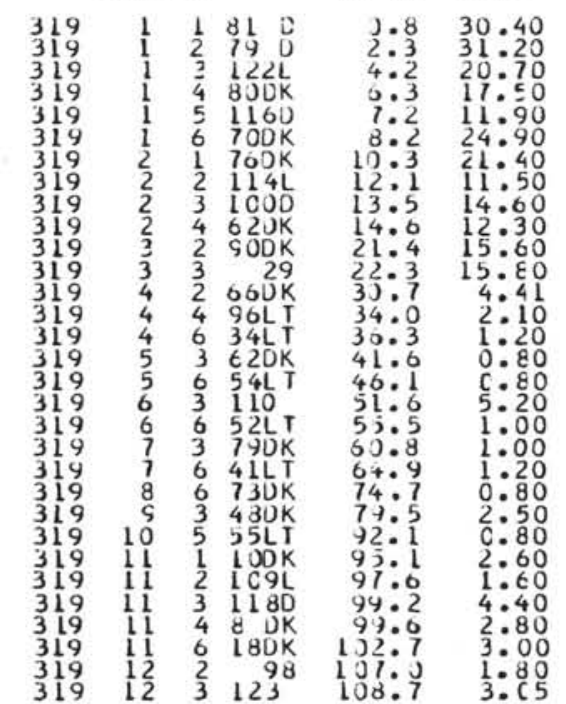
0.24
$0: 20$
$0: 14$
$0: 12$
$0: 05$
$0: 22$
$0: 07$
$0: 02$
$0: 05$
$0: 03$
$0: 05$
$00: 05$
$0: 03$
$0: 02$
$0: 03$
$0: 02$
$0: 00$
$0: 03$
$0: 01$
$0: 02$
$0: 01$
$0: 01$
$0: 03$
$0: 01$
$0: 03$
$0: 02$
$0: 04$
$0: 03$
$0: 03$
$0: 03$
0.03

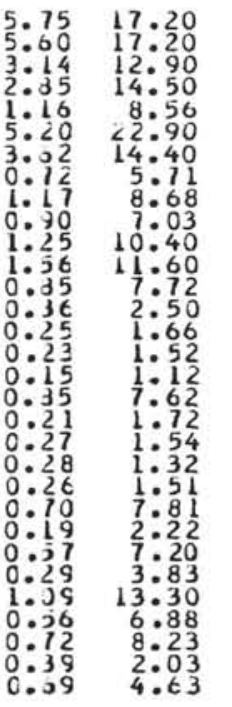
$5: 86$
$5: 26$
$2: 82$
$3: 37$
$1: 83$
$7: 51$
$2: 99$
$1: 19$
$1: 69$
$1: 34$
$1: 81$
$2: 03$
$1: 54$
$0: 45$
$0: 30$
$0: 34$
$0: 13$
$1: 54$
$0: 21$
$0: 24$
$0: 30$
$0: 36$
$2: 18$
$0: 21$
$1: 84$
$1: 18$
$4: 02$
$2: 00$
$2: 56$
$0: 39$
1.24 $\begin{array}{ll}2.67 & 3.71 \\ 2: 89 & 3: 64 \\ 1: 56 & 21: 80 \\ 1: 67 & 22: 90 \\ 1: 27 & 35: 50 \\ 2: 62 & 5: 57 \\ 2: 62 & 26: 40 \\ 1: 52 & 38: 10 \\ 1: 57 & 33: 90 \\ 1: 49 & 36: 20 \\ 1: 73 & 34: 70 \\ 1: 68 & 30: 80 \\ 0: 49 & 43: 70 \\ 0: 34 & 50: 60 \\ 0: 23 & 51: 80 \\ 0: 26 & 52: 10 \\ 0: 22 & 53000 \\ 0: 59 & 42: 10 \\ 0: 22 & 52: 50 \\ 0: 20 & 51: 10 \\ 0: 24 & 56: 20 \\ 0: 25 & 52: 30 \\ 0: 50 & 43: 00 \\ 0: 19 & 52: 50 \\ 0: 44 & 46: 10 \\ 0: 33 & 50: 00 \\ 0: 75 & 38: 90 \\ 0: 51 & 45: 60 \\ 0: 53 & 43: 50 \\ 0: 34 & 52: 10 \\ 0.46 & 46: 70\end{array}$ $1: 51$ K20 P205

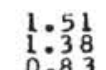
$1: 51$
$1: 38$
$0: 83$
$0: 74$
$0: 66$ 文: 268 $1: 09$
$1: 47$
0.60 2.16 0.72 0.30

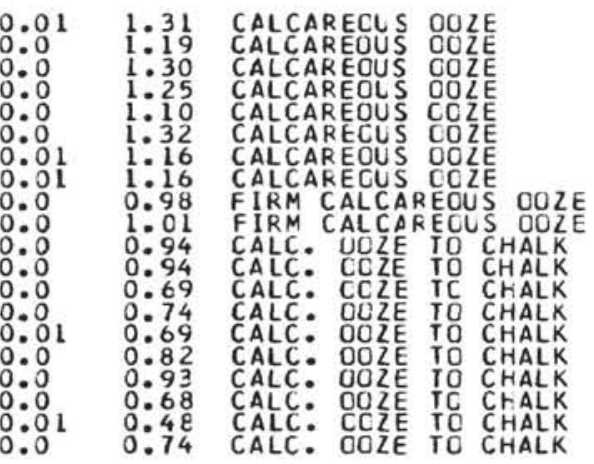

$\mathrm{CL}$ LITHCLCGY

7.62
$8: 07$
FERRUGINOUS CLAY $5: 93$ ZEOLITIC MARL

5:74 ZEELITIC MERL CLAY

6:07 CLAYEY NANNO ODLE 2.60
3
31
3 $3: 24$ CAYEY NANAO DOZE $4: 18$ CLAYEY NANNU DOZE 1.69 NANNU OOZE 08 NANNO OCZE 0.87 NANNO 002

1.07 NANNO OCZE

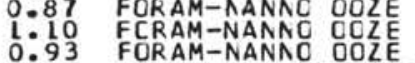
1.2 $1: 05$
0.81
0 1.48 FERRUG: NANA DOZE
$1: 21$ FERRUG: NANNO COZE $1: 22$
$1: 13$
$1: 22$
$1.2 R R U G$ 
SITE 3LI: LAT 12 DEG L MIN S; LONG $8 L$ DEG 54 MIN W; DEPTH 4817 M (ANAL LI)

SANPLE

$$
\text { DEPTH S102 }
$$
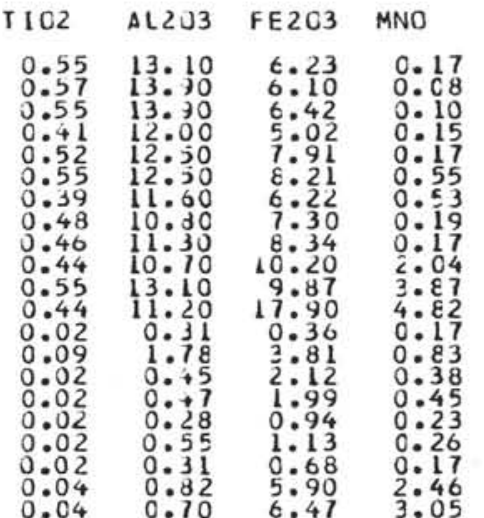

MGO

CAO
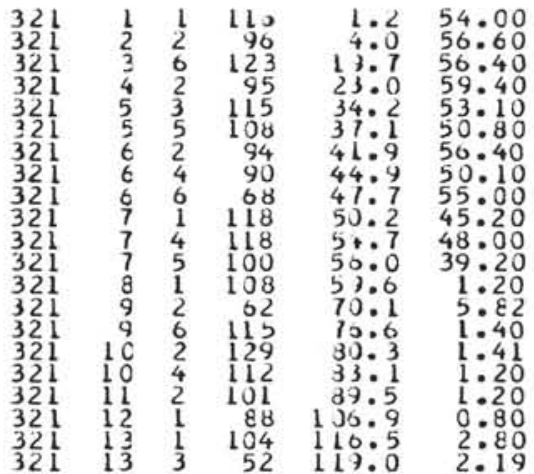

SITE 322: LAT 6C OES L MIN

SANPLE

S:

LCAG 79 OEG 25 MIN W: DEPTH $5036 \mathrm{M}$ (ANAL. hALLACE)
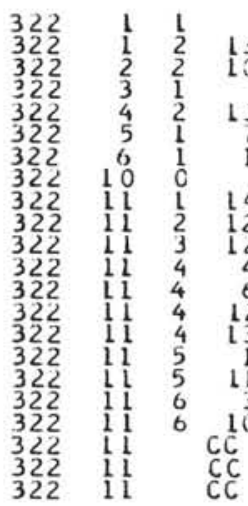

SITE $323:$ LAT
SAMPLE

CC 2 514:5 $54: 60$
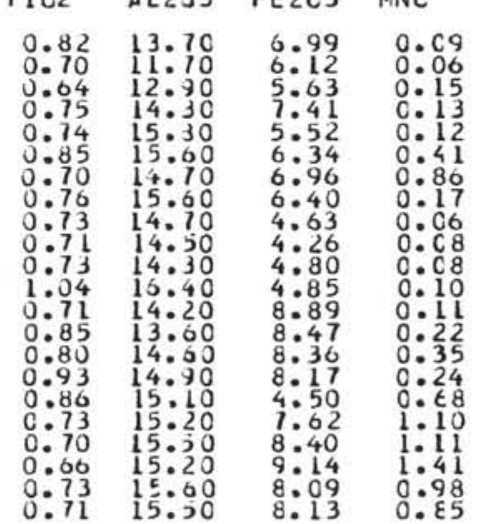

MG

$\frac{3}{3}: 043$

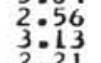

2.64

2.65
3.25

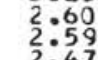

$\begin{array}{ll}2 & 2.59 \\ 1 & 2.47\end{array}$

2.47
2.11
3.66

3.66 0.95

3
3

2.81

2.85
2.65
2.69

$\begin{array}{lllll}8.13 & 0.98 & 2.69 & 1.20 & 1.55 \\ & .176 & 0.99 & 1.68\end{array}$

DEPTH SIVZ

CNG 9 S LEE

NIN W; DEPTH 5013 N (ANAL WALLACE)

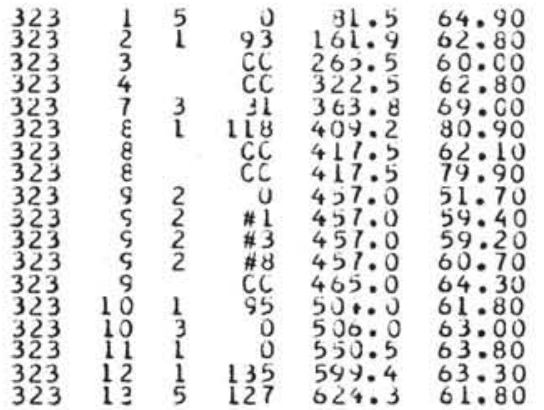

$\begin{array}{ll}0.62 & 13.00 \\ 0.64 & 13: 20 \\ 0: 70 & 14: 10 \\ 0: 67 & 14: 60 \\ 0.75 & 13: 90 \\ 0: 25 & 5: 50 \\ 0: 75 & 14: 50 \\ 0: 31 & 6: 90 \\ 0: 32 & 12: 70 \\ 0: 83 & 14: 90 \\ 0: 84 & 15: 30 \\ 0: 48 & 9: 90 \\ 0.63 & 13: 90 \\ 0.46 & 10: 00 \\ 0: 73 & 15: 70 \\ 0: 75 & 15: 40 \\ 0.76 & 16: 20 \\ 0.83 & 14.50\end{array}$

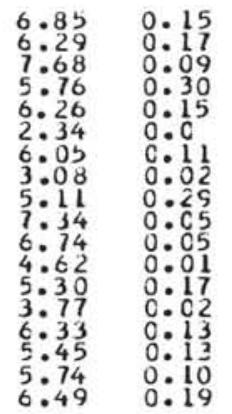

CAO

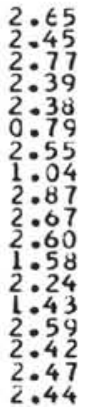

CAO

2.94
$2: 36$
$3: 03$

$\begin{array}{ll}4.88 & 3.52 \\ 3.28 & 2.88\end{array}$

$2.24 \quad 2: 45$

$.89 \quad 3.64$

$\begin{array}{ll}377 & 3.72 \\ .79 & 3.86\end{array}$

$\begin{array}{ll}1.42 & 2.64 \\ 1.61 & 2.07\end{array}$

1.:34

$1.09 \quad 1.81$

$\begin{array}{ll}1.13 & 1.68 \\ 0.20 & 1.55\end{array}$

P205

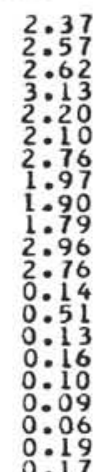

0.18

0.24

1.48
$0: 13$

0.37

0.26

1.51
2.07

0.08

0.16

0.84

0.10

0.25

K20 P205 CL

$\begin{array}{ll}2.09 & 0.23 \\ 1.82 & 0.11 \\ 1.83 & 0.18\end{array}$

$2.13 \quad 0.17$

2.3500 .27

2.66

1.46
1.64
1.79

$\begin{array}{ll}2.32 & 0: 23 \\ 4: 20 & 0: 11 \\ 2: 84 & 0: 24\end{array}$

$3: 04 \quad 0.33$

$4.26 \quad 0.30$

$4.65 \quad 0.40$

$\begin{array}{ll}4.51 & 0.55 \\ 3.57 & 0.54\end{array}$

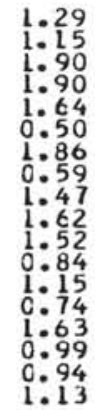

NA2C K

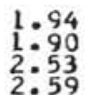

$1: 94$
$2: 90$
$2: 53$
$2: 17$
$2: 17$

K20 P20

2.48
$2: 90$
$2: 42$
$2: 75$

0.15
$0: 14$
0.32

0.32

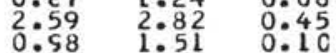

$2: 01$

2
2
$1: 54$
$1: 55$

$1: 55$

$: 40$
$1: 98$
1.89

$1: 86$

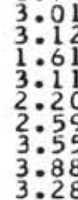

0.18

0:17

0.21

0.118

0.18

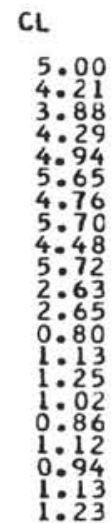

LITHCLCGY

SILICEOUS CLAY

SIL CLAY CHAY ASH ASH-R R I CH CAAY

ASH-R CH CEAY

S

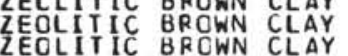

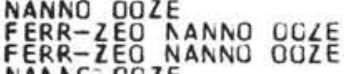

NANAC DOZE

12 NANND COZE

13
$1: 23$
$1.26 R R$ F NANAO CCZE

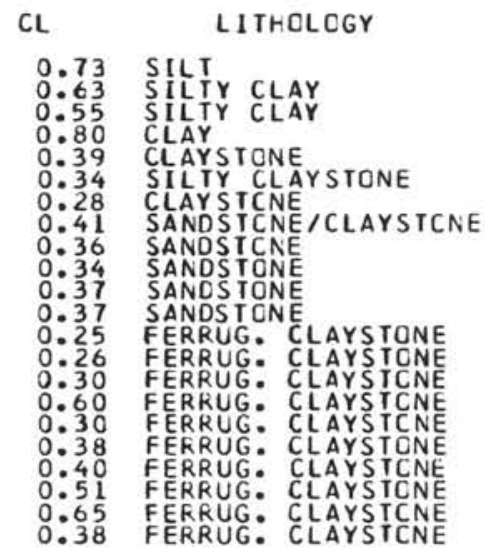

CL

LITHCLCGY

0.89 CIATCMACEOLS CLAY

1.23 DIATMMACEOLS CLAY

0.50 DIATCM. CLAYSTONE

0.50 CLAYSTCNE

$0: 81$ CLAYSICNE

0:40 CLAYSTCNE

0.26 CHERT 0 CLAYSTCNE

0.38 SILTY CLAYSTCNE
0.95 SIITY CLAYSTCNE

0.35
0.30 CLAYSICNE
0.34 CLYS 

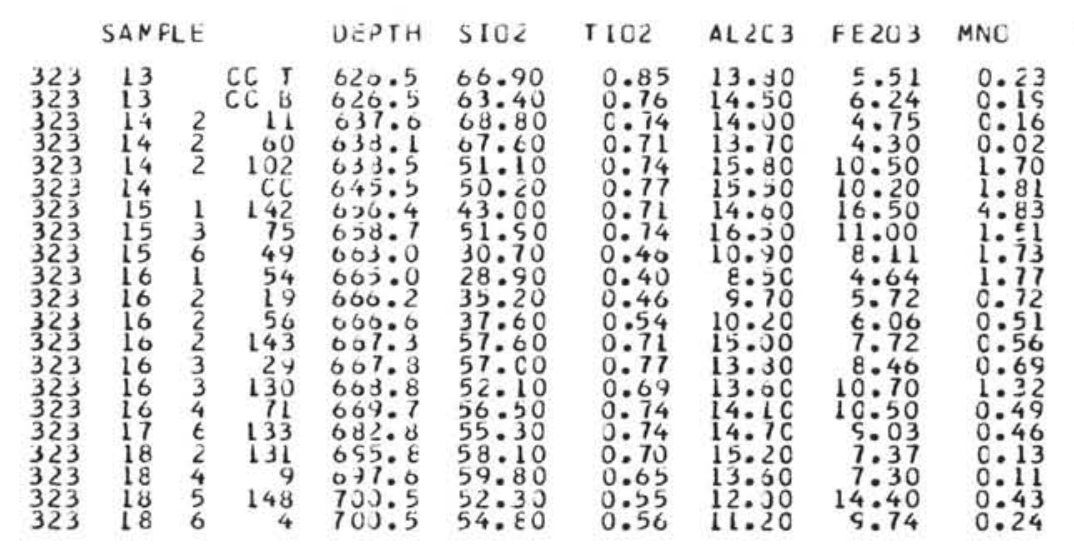

$M G O$

CAO

NA2C $\quad K 20$

P205

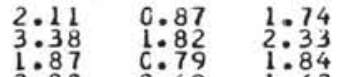

3.55
$3: 59$
$3: 15$
$3: 33$
2.61
2.34
2.74
$3: 23$
$2: 13$
1.98
2.04
2.18
3.03
2.28
2.06
2.66
2.80
3.51
$2: 75$
2.62
2.43

0.28

$\mathrm{CL}$

LITHCLCEY

SITE 32E: LAT 55 DEG $3 \mathrm{MIN} \mathrm{S:} \mathrm{LCAG} 73$ JEG $40 \mathrm{~N} I \Lambda$ W: DEPTH 3755 M (ANAL. WALLACE)

DEPTH SIOZ

TIO2 AL2U3 FE2U3 MNO

MGO

$\mathrm{CAO}$

NA2O

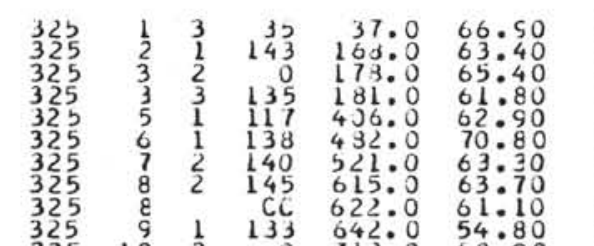

$\begin{array}{ll}0.74 & 13.00 \\ 0: 75 & 14.50 \\ 0.74 & 14: 00 \\ 0.65 & 13.00 \\ 0.77 & 14.70 \\ 0: 74 & 14: 70 \\ 0.72 & 14.00 \\ 0.71 & 13: 70 \\ 0.75 & 15.20 \\ 0.70 & 14.30\end{array}$

6.10
$5: 85$

0.17

2.65
2.60

3.15
$3: 18$
$3: 47$

2.82

6.25

0.15

2.76

$2: 78 \quad 2.56$

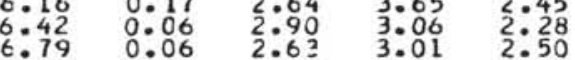

$\begin{array}{lllll}7.29 & 0: 09 & 2: 77 & 3: 52 & 2: 69 \\ 7.65 & 0: 15 & 3.59 & 2: 95 & 2: 71\end{array}$

102 O $113: 0$ \$8.9

$0: 79 \quad 16: 30$

7.43

$\begin{array}{llll}3.19 & 3.56 & 3.43\end{array}$

SANPLE

DEPTH SIU2

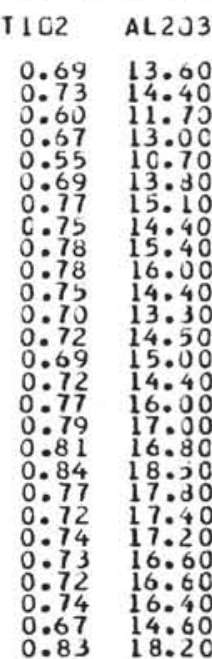

FE 203 MNC

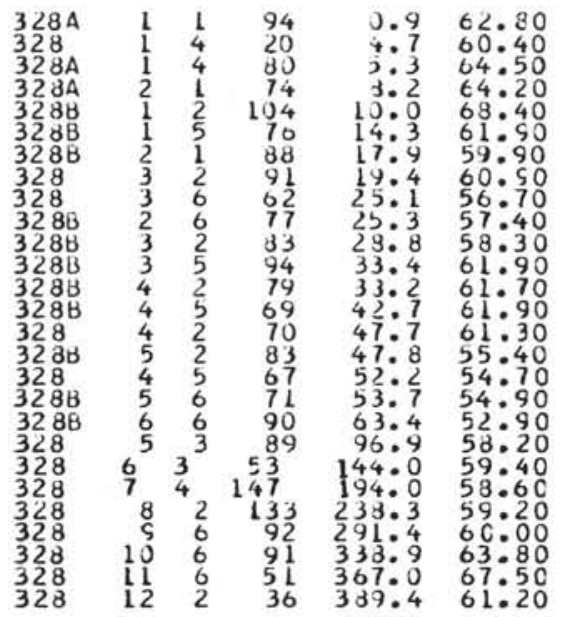

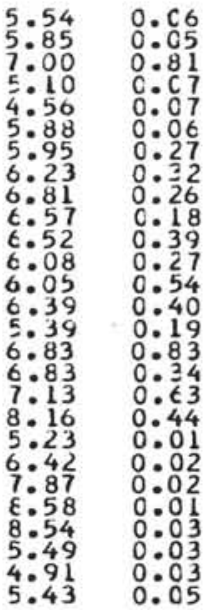

MGC

CAO

$\mathrm{NA2C} \quad \mathrm{K} 2 \mathrm{O}$

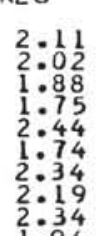

2.11
$2: 02$
1.88
$1: 75$
$2: 44$
$1: 74$
$2: 34$
$2: 19$
$2: 34$
1.96

$\begin{array}{ll}0.32 & \text { CLAYSIONE } \\ 0.27 & \text { CLAYSTCNE } \\ 0.22 & \text { CLAYSICNE }\end{array}$

0.26 CLAYSTCNE

0.34 FERRUC: CLAYSTCNE

0.58 FERRUG. CLAYSTONE

$0: 39$ FERRUG. CLAYSICNE

$0: 22$ FERRUG. CLAYSICNE

0.22 FERRUG: CLAYSICNE

$0: 53$ FERRUG: CLAYSTONE

0.24 FERRUG: CLAYSICNE

0.24 ZEC.FERRUG .CLAYSICNE

0.25 ZEC:FERRUG CLAYSTONE

$1.93 \quad 0.21$

CL

LITHCLOGY

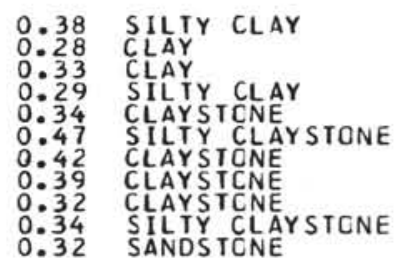

0.32 SILTY CLAYSTCNE

P205

$$
\text { CL }
$$

\begin{tabular}{|c|c|}
\hline & LITHO \\
\hline 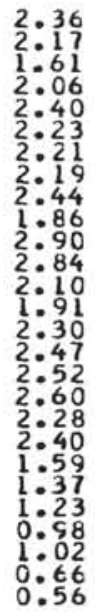 & 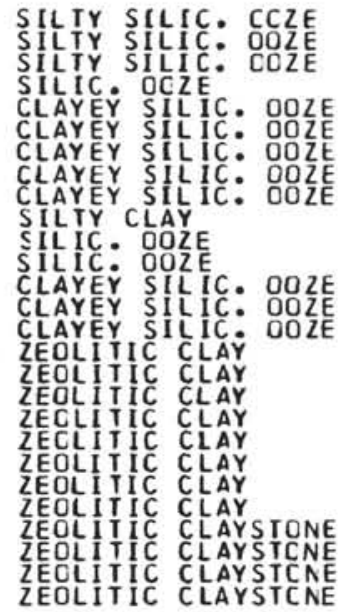 \\
\hline
\end{tabular}


SITE 330:LAT 50 DEG $55 \mathrm{MIA} \mathrm{S;} \mathrm{LCAG} 46$ DEG $53 \mathrm{MIN}$ W; OEPTH 2636 M (ANAL WALLACE) SAMPLE DEPTH SIO2 TIO2 AL2C3 FE2O3 MNO MGO CAO NAZO

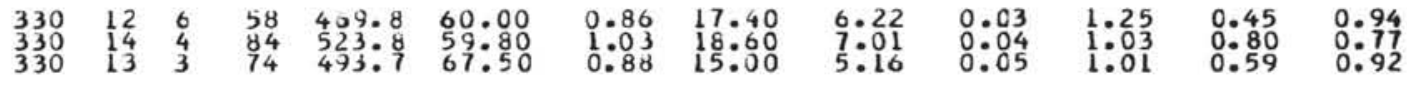
SITE 332: LAT 36 DEG 53 MIN A; LLAG 33 CEG 33 $\mathrm{MIN}$ W; CEPTH $1818 M$ (ANAL IERRANA) SAMPLE DEPTH SIO2 TIO2 AL2O3 FE2O3 MNO MGO CAO NAZO

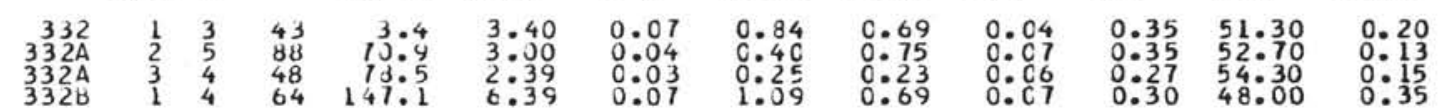
SITE 333: LAT 30 DEG $50 \mathrm{NIA} \mathrm{A;} \mathrm{LCNG} 33$ CEG $40 \mathrm{NIN} \mathrm{W;} \mathrm{CEPTH} \mathrm{1666M} \mathrm{(ANAL} \mathrm{TERRANA)}$ DEPTH SIO2 TIU2 AL2C3 FE2O3 MNO

MGO

CAO

NA2C

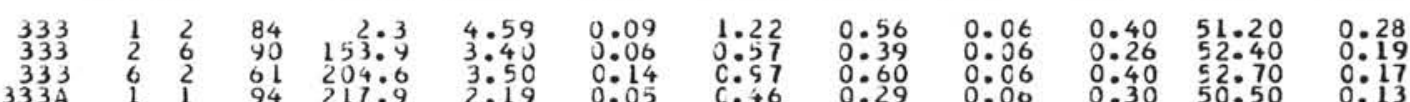

SITE 334: LAT 37 CEG 2 NIN N; LCAG 34 DEG 25 NIN W; DEPIH 2E32 M (ANAL TEFRANA)

DEPIH SIC2 TIO2 AL2O3 FE2O3 MNO

MGO

$\mathrm{CAO}$

NA2C

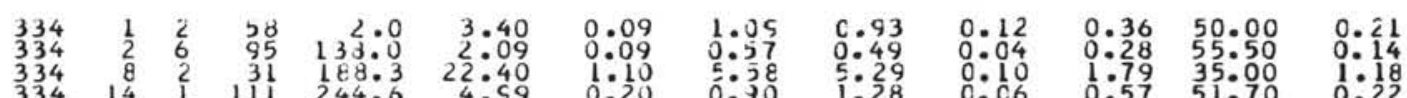

SITE 335: LAT 37 DEG $18 \mathrm{NIA} \mathrm{N;} \mathrm{LCNG} 35$ CEG $12 \mathrm{NIN}$ W; CEPTH $3198 \mathrm{~N}$ (ANAL TERRANA) SAMPLE DEPIH SIG2 TIC2 ALZL3 FEZO3 MNO MGO CAO NAZC K2O

SITE 338:LAT 67 DEG 47 NIN ; LLCAG 5 DEG 23 MIN E;DEPTH 131 9 M (ANAL hALLACE) SAMFLE DEPTH SIU2 TIU2 AL2O3 FE2O3 MNO $M G O$

CAD
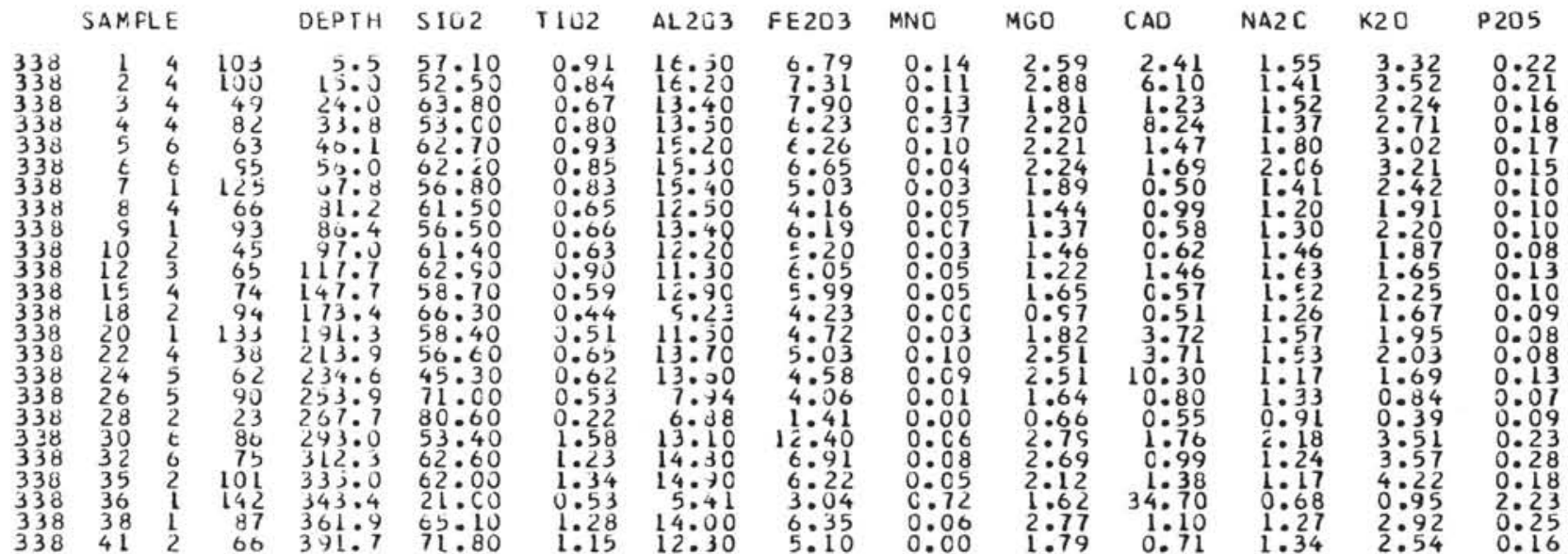

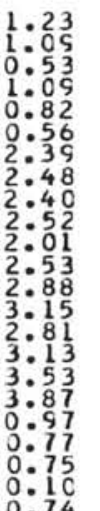

LITHCLCGY ILTY CLAY
ILIYY CLAY
ILIYY CLAY

\section{TRAM NANNC OOZE} 0
0
0
0
0
0.29

205

CL

LITHCLCGY

\section{$0.13 \quad 1.57$ FCRAM NANNC OOZE} $\begin{array}{llll}0.08 & 0.19 & 0.57 & \text { FCRAM NANNO DOZE } \\ 0.06 & 0.14 & 1.09 & \text { FORAM NANNC OCZE }\end{array}$

K20 P205 CL

LITHCLGGY

$0.14 \quad 0.14 \quad 1.70$ FCRAN NANAC OOZE

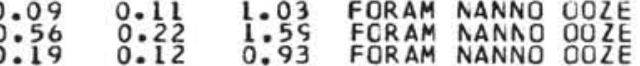

P205 CL

LITHCLCGY

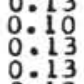

0.99 FCRAM NANAC DOZE 1.12
$0.83 R A M$
0 $1: 49$ FCRAM NANNO OCZE
0.98 FORAM NANAC COZE 
SITE 341 :LAT 67 DEG $20 \mathrm{NIN} \mathrm{N;LCNG} 6$ DEG 7 MIN E; DEPTH 1444 M (ANAL WALLACE)

\begin{tabular}{|c|c|c|c|c|c|c|c|c|c|c|c|c|c|c|}
\hline SANPL & & DEPTH & SIU2 & T IC2 & $A L 2 C 3$ & FE203 & MNO & MGO & CAO & $\mathrm{NA} 2 \mathrm{C}$ & $\mathrm{K} 2 \mathrm{O}$ & P205 & $\mathrm{CL}$ & L ITHCLCGY \\
\hline $\begin{array}{l}1 \\
4 \\
5 \\
7 \\
5 \\
11 \\
13 \\
16 \\
18 \\
20 \\
22 \\
24 \\
25 \\
26 \\
27 \\
28 \\
29 \\
30 \\
31 \\
32 \\
33 \\
34\end{array}$ & $\begin{array}{cc}t & 90 \\
2 & 98 \\
6 & 65 \\
5 & 45 \\
3 & 58 \\
4 & 84 \\
1 & 123 \\
1 & 131 \\
1 & 106 \\
1 & 82 \\
6 & 92 \\
1 & 94 \\
6 & 90 \\
6 & 90 \\
6 & 50 \\
6 & 85 \\
6 & 85 \\
6 & 84 \\
6 & 75 \\
5 & 92 \\
6 & 73 \\
6 & 58 \\
6 & 86\end{array}$ & $\begin{array}{r}8: 5 \\
31: 5 \\
40: 2 \\
03: 5 \\
19: 0 \\
100: 3 \\
100: 8 \\
162: 5 \\
202: 1 \\
245: 8 \\
270: 4 \\
321: 9 \\
341: 0 \\
359: 6 \\
373: 9 \\
397: 9 \\
407: 3 \\
416: 8 \\
429: 9 \\
435: 7 \\
443: 1 \\
434: 9\end{array}$ & $\begin{array}{l}62: 20 \\
55: 60 \\
60: 80 \\
60: 00 \\
62: 80 \\
59: 40 \\
59: 30 \\
60: 00 \\
60: 50 \\
52: 90 \\
60: 20 \\
65: 40 \\
61: 10 \\
48: 80 \\
52: 60 \\
59: 60 \\
63: 50 \\
60: 30 \\
61: 70 \\
61: 90 \\
62: 10 \\
61: 20\end{array}$ & $\begin{array}{l}0.60 \\
0.00 \\
0: 55 \\
0: 80 \\
0: 83 \\
0: 77 \\
0: 70 \\
0: 74 \\
0: 72 \\
0.94 \\
0.85 \\
0.87 \\
0: 91 \\
0: 66 \\
0.63 \\
0.71 \\
0: 66 \\
0: 91 \\
0: 82 \\
0.76 \\
0: 76 \\
0.73\end{array}$ & $\begin{array}{l}13.10 \\
13: 200 \\
1 c: 90 \\
14: 600 \\
14: 90 \\
13: 10 \\
15: 30 \\
14: 50 \\
14: 50 \\
15: 30 \\
16: 200 \\
15: 30 \\
16: 30 \\
13: 40 \\
13: 20 \\
14: 30 \\
11: 70 \\
17: 20 \\
15: 70 \\
15: 30 \\
14: 30 \\
14.50\end{array}$ & $\begin{array}{l}5: 44 \\
5: 58 \\
4: 14 \\
5: 08 \\
5: 97 \\
5: 93 \\
5: 94 \\
5: 73 \\
5: 57 \\
7: 39 \\
6: 24 \\
6: 89 \\
5: 42 \\
6: 49 \\
7: 30 \\
4: 31 \\
5: 33 \\
5: 49 \\
5: 52 \\
5: 81 \\
5: 27 \\
5: 56\end{array}$ & $\begin{array}{l}0: 16 \\
0: 04 \\
0: 03 \\
0: 05 \\
0: 07 \\
0: c 8 \\
0: 10 \\
0: c 7 \\
0: 02 \\
0: 06 \\
0: 08 \\
0: 07 \\
0: 01 \\
0: 02 \\
0: 02 \\
0: 01 \\
0: 04 \\
0: 05 \\
0: 02 \\
0: 01 \\
0: 01 \\
0: 01\end{array}$ & $\begin{array}{l}2.13 \\
2: 47 \\
1.27 \\
2: 15 \\
2: 28 \\
2: 68 \\
2.63 \\
2.61 \\
2: 60 \\
2.83 \\
2.74 \\
2.21 \\
11.56 \\
1.68 \\
1.61 \\
1.57 \\
1.32 \\
1.87 \\
1.45 \\
1.59 \\
1.60\end{array}$ & $\begin{array}{l}5.17 \\
4: 50 \\
0: 34 \\
4: 22 \\
3: 47 \\
4: 87 \\
5: 13 \\
5: 38 \\
5: 08 \\
5: 47 \\
2: 56 \\
0: 75 \\
0: 32 \\
5: 67 \\
5: 86 \\
3: 69 \\
1: 23 \\
0: 38 \\
0: 28 \\
0: 31 \\
0: 49 \\
0: 38\end{array}$ & $\begin{array}{l}1: 30 \\
1: 26 \\
1: 23 \\
1: 51 \\
1: 76 \\
1: 83 \\
1: 94 \\
1: 57 \\
1: 41 \\
1: 63 \\
1: 59 \\
1: 25 \\
0: 02 \\
1: 15 \\
1: 24 \\
1: 22 \\
1: 30 \\
1: 56 \\
1: 53 \\
1: 40\end{array}$ & $\begin{array}{l}3.09 \\
2: 66 \\
1: 63 \\
2: 74 \\
2: 78 \\
2: 93 \\
3: 16 \\
2: 88 \\
2: 77 \\
2: 94 \\
3: 41 \\
3: 05 \\
2: 68 \\
1: 89 \\
2: 00 \\
2: 17 \\
1: 75 \\
2: 53 \\
2: 45 \\
1: 99 \\
2: 15 \\
2: 06\end{array}$ & $\begin{array}{l}0.18 \\
0: 14 \\
0: 07 \\
0: 15 \\
0: 18 \\
0: 18 \\
0: 19 \\
0: 18 \\
0: 17 \\
0: 17 \\
0: 15 \\
0: 18 \\
0: 09 \\
0: 09 \\
0: 08 \\
0: 08 \\
0: 07 \\
0: 10 \\
0: 10 \\
0: 09 \\
0: 11 \\
0: 09\end{array}$ & $\begin{array}{l}0.89 \\
1: 69 \\
2: 35 \\
0: 75 \\
0: 46 \\
0: 46 \\
0: 57 \\
0: 46 \\
0: 46 \\
0: 82 \\
0: 56 \\
0: 46 \\
0: 84 \\
0: 98 \\
0: 96 \\
1: 11 \\
1: 13 \\
0: 80 \\
0: 83 \\
1: 10 \\
0: 78 \\
0.79\end{array}$ & 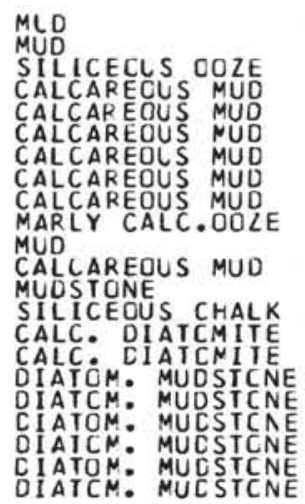 \\
\hline
\end{tabular}

SITE 344:LAT 76 UEG $\rightarrow$ NIN N;LCNG 7 CEG 53 NIN E;DEPTH $2201 \mathrm{~N}$ (ANAL MALLACE)
SAMPLE
DEPTH SIC2
IIU2 ALLO3 FE2O3 MNO
MGO
CAO
NA2O K2O
P205 CL
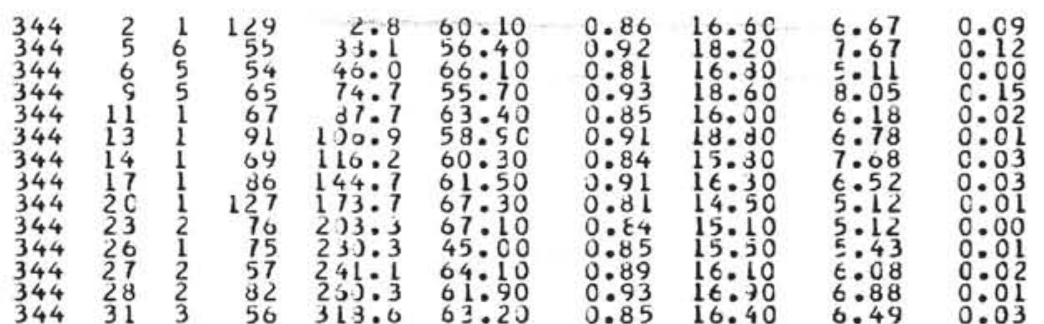

$\begin{array}{ll}2.07 & 1.04 \\ 1.81 & 0.89 \\ 1.69 & 0.48 \\ 2.49 & 0.81 \\ 2.04 & 0.69 \\ 2.28 & 0.91 \\ 2.06 & 0.86 \\ 2.44 & 1.34 \\ 1.73 & 0.86 \\ 1.79 & 0.61 \\ 2.00 & 0.98 \\ 2.02 & 0.70 \\ 2.30 & 0.69\end{array}$

$\begin{array}{ll}1.57 & 2.65 \\ 1: 50 & 2: 87 \\ 1: 70 & 2.70 \\ 1: 36 & 3: 11 \\ 1: 71 & 3.21 \\ 1: 53 & 2.95 \\ 1: 60 & 2.69 \\ 2: 30 & 3.27 \\ 1: 99 & 2.53 \\ 1.89 & 2.69 \\ 1: 56 & 2.75 \\ 1: 75 & 2.78 \\ 1.75 & 2.92\end{array}$

$344 \quad 31 \quad 3 \quad 56 \quad 313: 0 \quad 63: 20 \quad 0.85 \quad 16: 40$

SITE 346:LAT 6Y UEG is MIN N;LCNG 8 DEG $41 \mathrm{~N}$ (N h; DEPIH $741 \mathrm{M}$ (ANAL hALLACE)

$$
\text { SAMPLE }
$$

DEPTH SIOZ

102 AL2C3 FE2O3 MNO

MGO

CAO

NA2C K2O
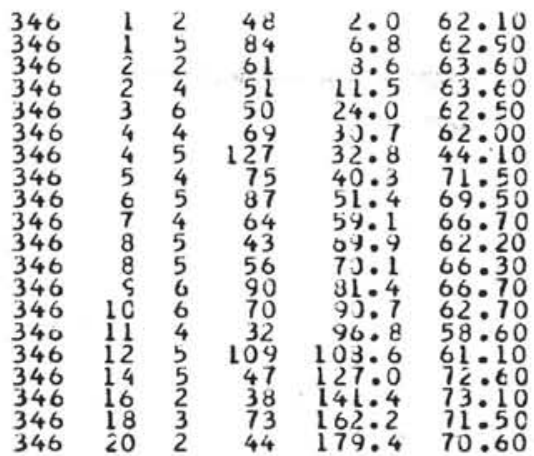

$\begin{array}{ll}0.74 & 12.30 \\ 1: 15 & 14: 90 \\ 0: 82 & 13: 30 \\ 1: 06 & 15: 80 \\ 1: 14 & 14: 90 \\ 1: 15 & 15: 40 \\ 1: 54 & 10: 50 \\ 0: 68 & 10: 60 \\ 0: 74 & 12: 20 \\ 0: 85 & 13: 30 \\ 1: 60 & 11: 10 \\ 1: 01 & 14: 20 \\ 0: 95 & 13: 10 \\ 0: 98 & 13: 30 \\ 1: 32 & 14: 50 \\ 1: 05 & 14: 00 \\ 0: 54 & 12: 80 \\ 0: 97 & 12: 50 \\ 0: 96 & 12: 30 \\ 1.08 & 13: 10\end{array}$
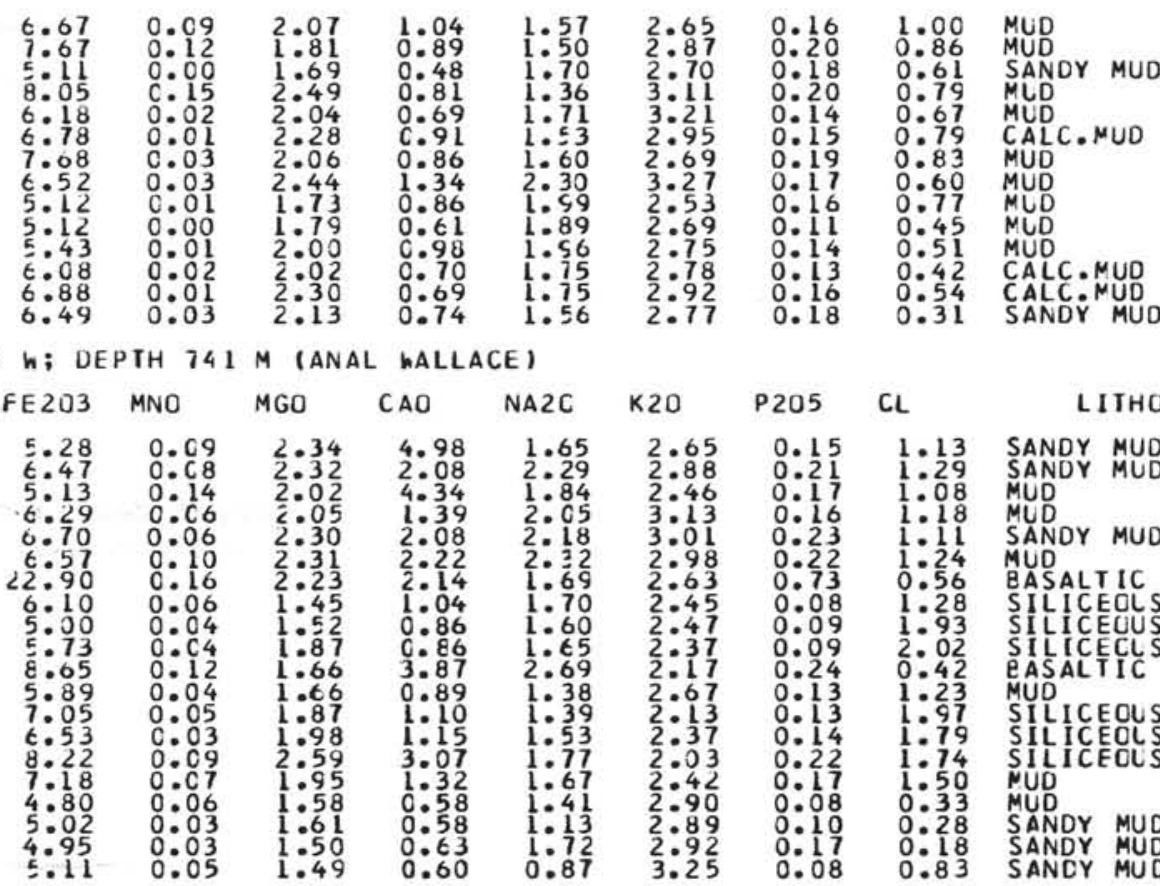
SITE 348:LAT GO DEE 30 MIN N;LCNG 12 DEE 23 MIN W;DEPTH 1777 M (ANAL WALLACE)

\begin{tabular}{ccc}
\multicolumn{3}{c}{ SAMPLE } \\
8 & 1 & 6 \\
8 & 3 & 1 \\
8 & 4 & 3 \\
8 & 5 & 3 \\
8 & 6 & 1 \\
8 & 6 & 4 \\
8 & 7 & 5 \\
8 & 8 & 4 \\
8 & 11 & 5 \\
8 & 13 & 3 \\
8 & 14 & 3 \\
8 & 15 & 1 \\
8 & 15 & 6 \\
8 & 21 & 6 \\
8 & 23 & 6 \\
8 & 25 & 6 \\
8 & 27 & 4 \\
8 & 29 & 6 \\
\hline & 3 & 5
\end{tabular}

$$
\text { DEPIH SIO2 IIO2 }
$$

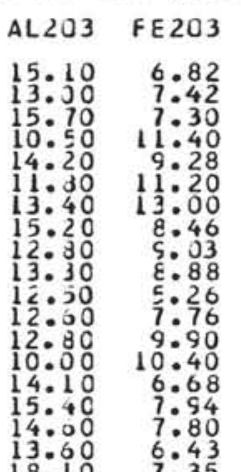

MNC
$0: 15$
$0: 13$
$0: 01$
$0: 10$
$0: 13$
$0: 14$
$0: 16$
$0: 05$
$0: 08$
$0: 07$
$0: 04$
$0: 03$
$0: 01$
$0: 74$
$0: 00$
$0: 03$
$0: 05$
$0: 02$
0.08

MGO

CAO NA2O K2O

P205

0.20

3.22
$2: 82$
3

$2: 50$
$1: 43$
$2: 03$
0.49
$2: 73$

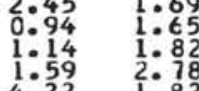

$\begin{array}{lll}69 & 06: 7 & 52: 90 \\ 64 & 11: 1 & 56: 90\end{array}$

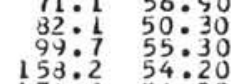

$\frac{1}{2}: 16$

$45 \quad 174.0 \quad 54.30 \quad 1: 68$

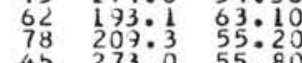

0.64

$\begin{array}{ll}292: 5 & 38.50 \\ 531.0 & 64.80\end{array}$

$\begin{array}{ll}373.1 & 62: 20 \\ 431: 9 & 63: 90 \\ 491: 9 & 65: 50\end{array}$

$\begin{array}{lll}1: 31 & 15: 40 & 7: 94 \\ 1: 24 & 14: 00 & 7: 80 \\ 1: 21 & 13: 50 & 6: 43 \\ 1.66 & 18.10 & 7: 35\end{array}$

0.02

4.66

2.44
3.02
2.49

$5.75 \quad 2.72$

2.26

$2: 36$
$1: 72$

$\begin{array}{ll}1.24 & 2.62 \\ 2.43 & 1.72\end{array}$

$\begin{array}{ll}4.23 & 1: 92 \\ 4.91 & 16.00\end{array}$

2.31
1.62

$1: 32$

$\begin{array}{llll}42 & 1.25 & 1: 20 & 0.51 \\ 28 & 1.36 & 1: 31 & 2.35\end{array}$

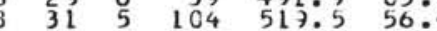
10.64

SAMPLE

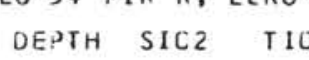

II W; DEPTH 4052 M(ANAL WALLACE)

$\begin{array}{llll}354 & 1 & 2 & 104 \\ 354 & 2 & c & \\ 354 & 3 & 1 & 10 \\ 354 & 4 & 1 & \\ 354 & 4 & 6 & 1 \\ 354 & 4 & 1 & \\ 354 & 5 & 1 & 9 \\ 354 & 6 & 3 & 74 \\ 354 & 7 & 4 & 3 \\ 354 & 8 & 3 & 40 \\ 354 & 5 & 5 & 9 \\ 354 & 10 & 6 & \\ 354 & 11 & 6 & 19 \\ 354 & 12 & 6 & 56 \\ 354 & 13 & 6 & 51 \\ 354 & 14 & 6 & 5 \\ 354 & 15 & 3 & 69 \\ 354 & 16 & 6 & 79 \\ 354 & 17 & 3 & \\ 354 & 18 & 6 & 55\end{array}$

$\begin{array}{rl}18.30 & 7.35 \\ 15: 00 & 5: 80 \\ 11: 80 & 4: 10 \\ 11: 90 & 4: 49 \\ 14: 30 & 5: 51 \\ 12: 30 & 4: 46 \\ 10: 70 & 3: 98 \\ 6: 49 & 2: 20 \\ 6: 39 & 2: 10 \\ 5: 71 & 2.04 \\ 6: 96 & 2.05 \\ 6: 11 & 1: 94 \\ 6: 20 & 2.21 \\ 5: 20 & 1.81 \\ 5: 20 & 1: 78 \\ 4: 49 & 1.72 \\ 8: 10 & 2: 76 \\ 7.34 & 2.75\end{array}$

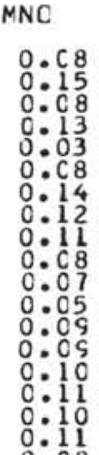

MGO

CAO

NA2C

K20

2.52

1.66
1.80
2.03

2.03
1.87
1.44

0.45

0.47

0.46

0.46

0.41

0.84

0.87

JEE 34 NIN W: DEPIt $2109 \mathrm{M}$ (ANAL WALLACE)

SAMPLE

DEPTH SIOZ TIU2

$\triangle L 2 J 3$ FE2O3 MN

$\begin{array}{rr}357 & 1 \\ 357 & 2 \\ 357 & 2 \\ 357 & 3 \\ 357 & 3 \\ 357 & 4 \\ 357 & 5 \\ 357 & 6 \\ 357 & 9 \\ 357 & 12 \\ 357 & 15 \\ 357 & 17 \\ 357 & 19 \\ 357 & 20 \\ 357 & 22 \\ 357 & 24 \\ 357 & 27 \\ 357 & 28 \\ 357 & 30 \\ 357 & 32 \\ 357 & 36 \\ 357 & 39 \\ 357 & 42\end{array}$

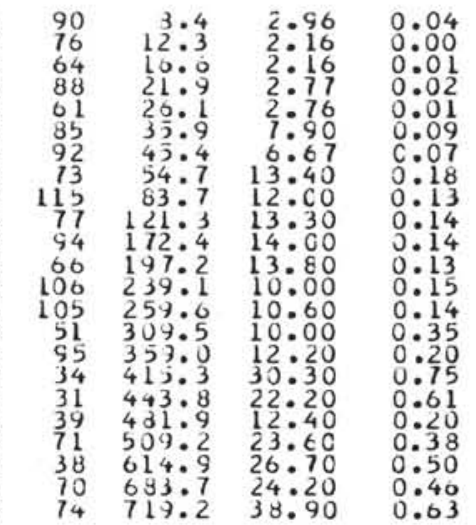

$\begin{array}{ll}1.09 & 0.48 \\ 0: 64 & 0: 26 \\ 0: 64 & 0: 31 \\ 0: 19 & 0: 35 \\ 0: 33 & 0: 34 \\ 2: 36 & 0: 93 \\ 1: 99 & 0: 81 \\ 3: 95 & 1: 60 \\ 3: 946 & 1: 22 \\ 3: 45 & 1: 27 \\ 3: 43 & 1: 39 \\ 3: 32 & 1: 52 \\ 2: 48 & 1: 08 \\ 2: 71 & 1: 22 \\ 2: 72 & 1: 52 \\ 2: 71 & 3.13 \\ 9: 34 & 3: 46 \\ 5: 33 & 2: 30 \\ 3: 57 & 1: 53 \\ 6: 71 & 2: 92 \\ 7: 84 & 3.76 \\ 5: 77 & 3: 17 \\ 9.06 & 5: 12\end{array}$

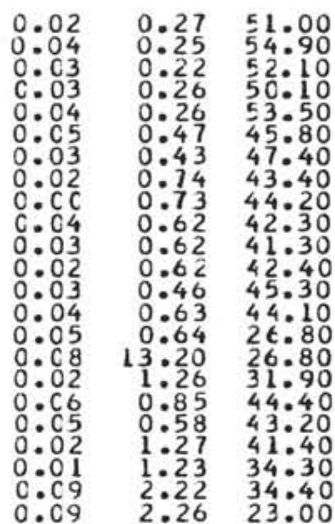

$\mathrm{N} 42 \mathrm{O}$

K20

P205

0.17 - 0.08

$\mathrm{CL}$

LITHCLOGY

0.16 1.83 MuD

$1: 09$ VOLCANICAST

0.28 0.5 EASALIIC ASH

$2: 16$

$0.1423: 03$ Mu

0.12 1.76 VOLCANIC ASH

0.1

0.190 .89 CLAYSTCNE

0.140 .71

0.40

0.52

MUDSTCNE

MUDSICNE

$205 \mathrm{CL}$

LITHCLOGY

0.15
$0: 14$
$0: 11$
$0: 11$
$0: 14$
$0: 12$
$0: 10$
$0: 09$
$0: 08$
$0: 11$
$0: 09$
$0: 08$
$0: 09$
$0: 10$
0.09
0.11
0.11

2.47

RAN-NANAC OOLE

1.48 MARLY OOZE

101 FORAM-NANNC OOZE

85

man

MARLY CHALK

0.56 NANAOFCAM CHALK

0.63 NANNO-FCRAM CHALK

0.36 ZECLIIIC MERLY CHALK

0.56 MEC DIAT NNARYY CHAL

0.24

0.43 MARLY NANNC CHALK

.31 MARLY NANNC CHALK

0.30 FERRUG: MARLY CHALK

0.42
$0: 26$
0.07

0.36

0.42
$0: 35$
0.57

0.54

0.42

0.49

0.380 .66

(6.

0.61

1.36
$0: 86$
$0: 39$
$0: 66$
0.48

$0.48 \quad 2.03$

$0 .: 51$

$0: 67$

1.78
2.03

0.12

0.39

0.64

.68

0

0.16

$0: 13$

$0: 33$

0

$0: 12$

1.00

LITHCLOGY

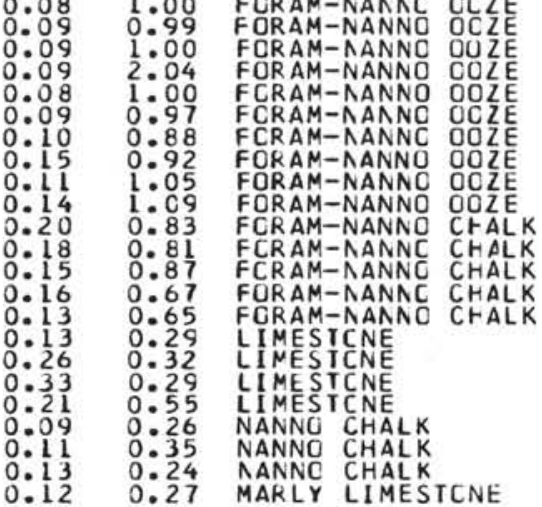


$\mathrm{NA2C}$

$\mathrm{k} 2 \mathrm{C}$

F 205

CL

LITHCLCGY

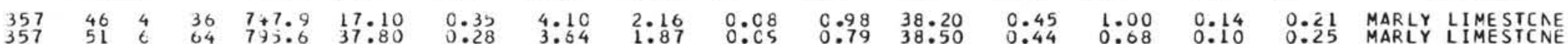
SITE 358: LAT 3I DEG 39 MIA S; LCNG 35 CEG 53 HIN W; CEPTH 4990 M (ANAL WALLACE) SAMPLE

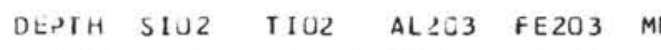

MGO
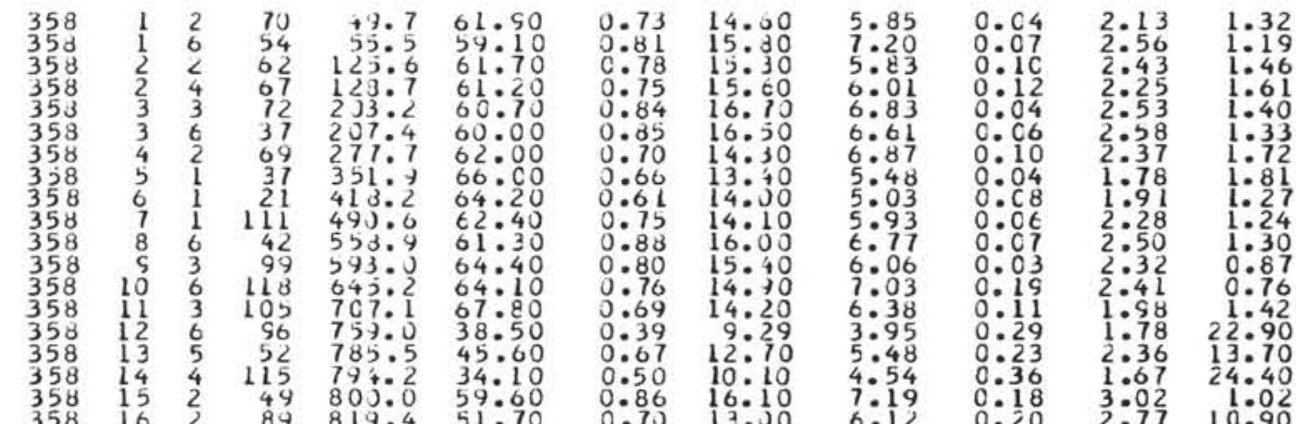

$$
\mathrm{NA2C}
$$

K20 P205

SITE 360: LAT 35 OEG 51 MIN S; LONG 13 LEG 6 NIN E; DEPTH 2577 M (ANAL WALLACE)

SAMPLE

DEPTH SIOZ
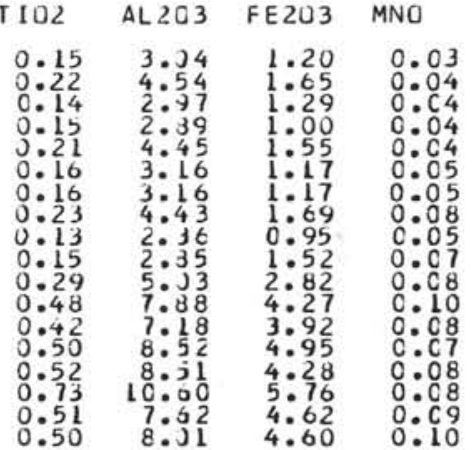

MGO CAO

$\begin{array}{ll}0.57 & 45.10 \\ 0.74 & 38.10\end{array}$

NA2O

0.36

$0.57 \quad 44.10$

$0.60 \quad 42.80$

$0.41 \quad 46.40$

$0.93 \quad 35.3000 .43$

$1.53 \quad 33.6$

25.20

$\begin{array}{lllll}36 & 4 & 7 & 573 & 1 \\ 6 & 27.40\end{array}$

$\begin{array}{lll}360 & 42 & 4 \\ 360 & 45 & 5\end{array}$

$\begin{array}{llllll}360 & 48 & 5 & 81 & 798: 8 & 31 \\ 360 & 50 & 2 & 72 & 832: 2 & 30.50\end{array}$

0.508 .31

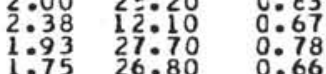

SITE 362: LAT

$$
\text { DEPTH SIOZ }
$$

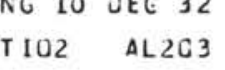

IA E;DEPIH $1336 \mathrm{~N}$ (ANAL TERRANA)
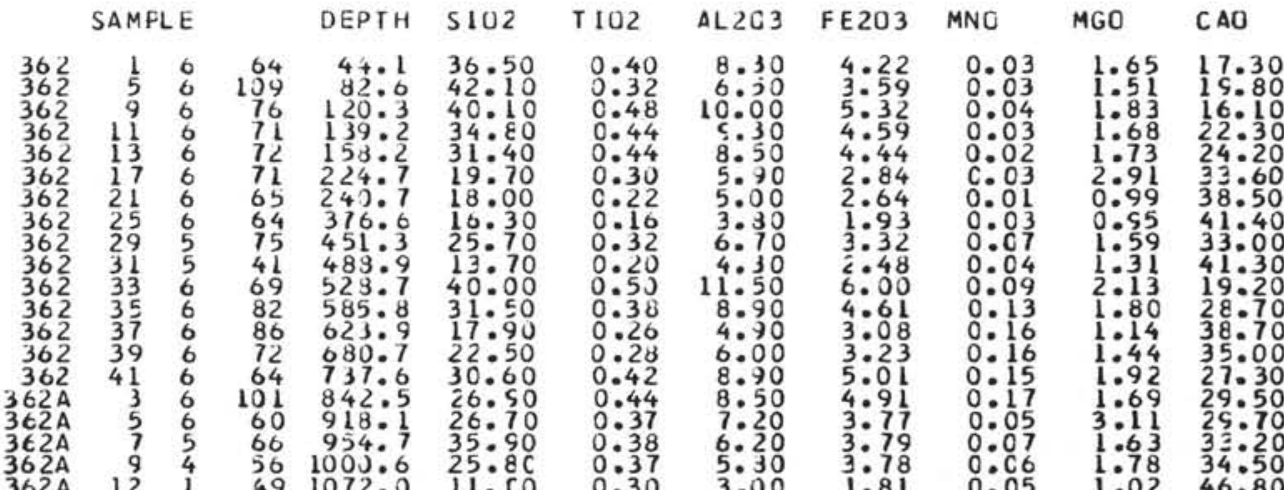

NA2C

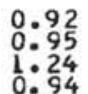

0.92
$0: 95$
$1: 24$
$0: 94$

0.94
$0: 95$
$1: 10$
0.27

$0: 78$

0.78
0.61
1.08

0.79

0.57

$1: 00$

c: $: 12$

2.72
$2: 88$
$2: 80$
2.62
3.04
$2: 96$
2.62
$2: 17$
$2: 90$
2.41
$2: 70$
2.98
$2: 98$
1.55
1.38
$3: 29$
2.33
4.25
2.97

0.11 $0: 10$ 0.13 0.120 0.25 0.14 0.12 0.19 0.16 $0: 14$ .17

K20 P205 CL $0.59 \quad 0.11$ $\begin{array}{lll}0 & 1 & 1 \\ 0 & 1 & 3 \\ 0 & 1 & 2 \\ 0 & 12\end{array}$ 0.56

0.67 $0: 90$ 0.45 1.00 1.50 $0: 13$ 2.01
1.42
1.55

$0: 1$ 0:12 0. 10 0.11 0.12 0.12 K2C P205
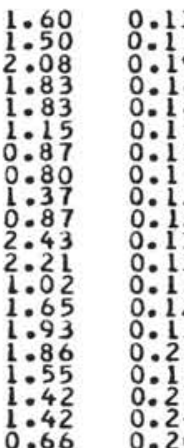

$\mathrm{CL}$
L ITHCL OGY $\begin{array}{ll}2.27 & \text { DIATCNACEOLS MUD } \\ 1: 72 & \text { DIATOMACEOLS MUD } \\ 1.58 & \text { DIATCNACECLS MUD }\end{array}$ 1.58 DIATCMACECLS MUD 1.14 SILICEOUS MUD 1.32 VCLC. RAD MUDSTCNE $1: 33$ SADICLARIAN MUDSTON 0.57 SILICECLS NUDSICNE 0.38 SILICECUS MUDSTCNE
1.89 SILICECLS MUDSTCNE 0.29 FERRUGINOUS MUOSTON 0.26 FERRUG: MARLY CHALK 0.26 FERRUG MARLY CHALK 0.25 FERRUGINOUS MLDSTONE

O.98 NANNO OOZE

0.78 NANNO COZE

0.87 NANNO CHALK

0.66 NANNO CHALK

0.67 AANNO CHALK

0.38 NANNO CHALK

0.37 MARLY NANAC CHALK

0.38 MARLY NANNC CHALK

0.22 MARLY NANNC CHALK 0.21 MARLY AANNC CHAL

LITHCLCGY

2.23 MARLY SIL CALC,OOZE

$1: 18$ MARLY CIATCM.OOZE
1.06 MARLY DIATCN.COZE

0.12 MARLY SIL.CALC.DOZ

1.23 MARLY CHALK

0.65 MARLY CHALK

0.6

$0: 43$ MARLY CHALK

0.32 MARLY CHALK

0.23 ERAARUD. CHALK

0:13 MARLY CHALK

0.07 MARLY LIMESTONE 
SITE 364: LAT 11 DEG 34 MIN S; LCNG 11 CEG 58 NIN E;DEPTH 2449 M (ANAL WALLACE) SAMPLE DEPTH SIO2 TIU2 AL2O3 FE203 MNC MGO CAO NAZO
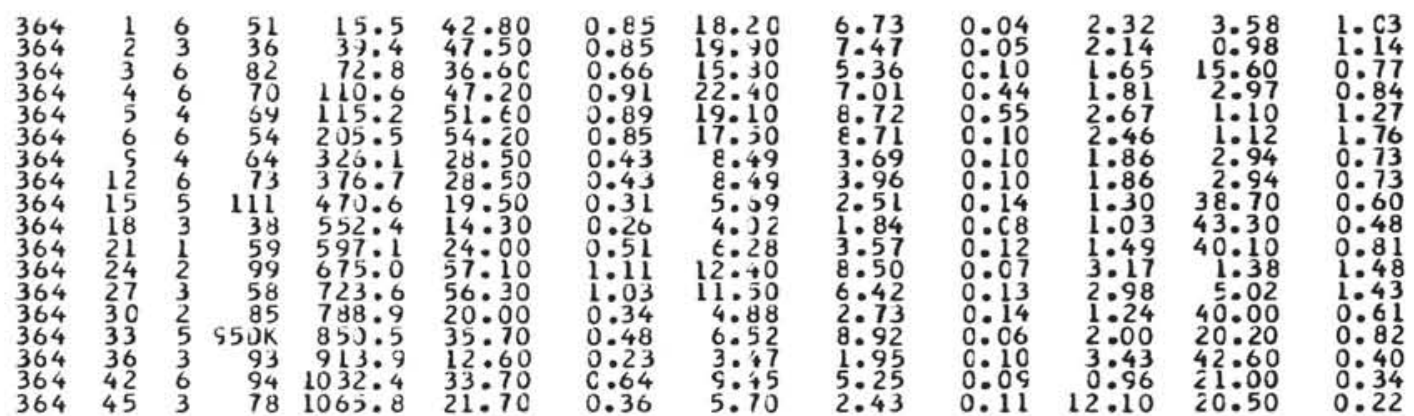
K20

P205

CL

LI THCLOGY

SITt $366: L A T 5$ UEG 41 MIA N; LCAG 19 DEG IL MIN W; DEPTH 2853 N (ANAL WALLACE)

$$
\text { SAMPLE }
$$

CAO NA2C

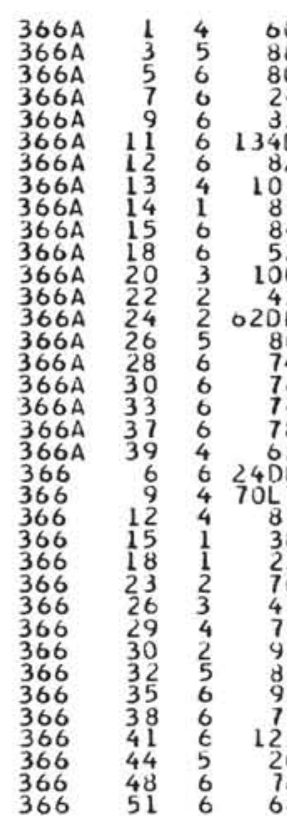

$$
\begin{aligned}
& \text { DEPIH SILZ } \\
& 5.120 .00
\end{aligned}
$$

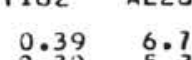

FE203 MNU

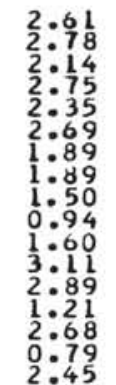

2.45
1.23

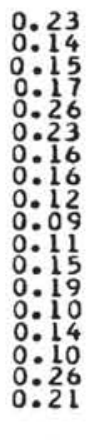

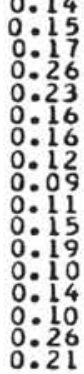

P205

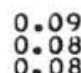

0.09
0.08
0.08

0.08

0.0

0.0

0.13

0.1

$0: 1$

$0: 09$

0.09

0.12

0:2

$0: 14$

0.14

$0: 08$

0.1

$0.09 \quad 0.28$

SILICEOUS LIMESICNE
SIMESICNE
0.45 SILICEOUS LIMESICNE

$0.10 \quad 0.37$ CLAYEY LIMESTCNE

0.15 0:26 CLAYEY SIMESICNE 0.14 O:18 MARLSIONE

\author{
$1: 25$ ODZE. BEAR ING NUD \\ IECL. BEARING MUC \\ NANNO CHALK \\ 0.49 MARLY CHALK \\ 0.39 MARLY CHALK \\ 0.47 MARLY CHALK \\ .52 LIMESTCNE \\ 30 MARLY DCLCNIIIIC LS.
}

NANNO MARL

NANNO MARL

NANNC

NANNO OOZE RAD. NANNC CHALK

CLAYEY CHALK

CLAYEY CHALK

CLAYEY CHALK

49 CLAYEY CHALK

6 NANNO CHALK

ANNO CHALK

NANN CHALK

PORC

$\begin{array}{lll}0.09 & 0.18 & \text { ARGILL: LIMESTCNE } \\ 010 & 0: 17 & \text { ARGILL: LINESTCNE } \\ 18 & 0: 17 & \text { ARGILL: LINESTCNE }\end{array}$ 
SITE 369: LAT 20 DEG 30 NIN N; LLNG 15 CEG O MIN W: DEPIH 1752 M (ANAL WALLACE)

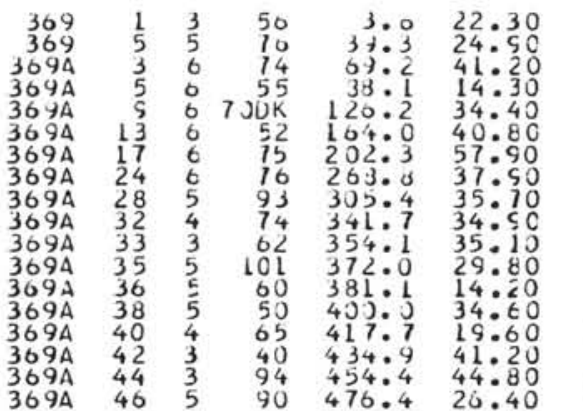

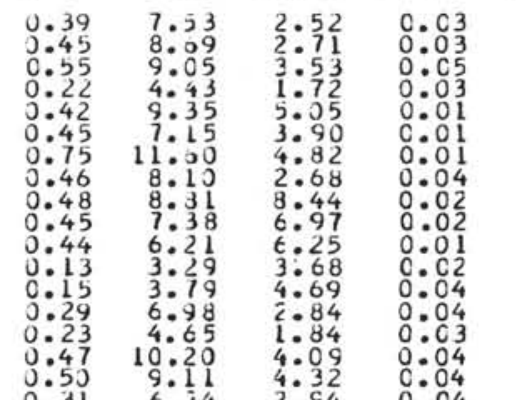
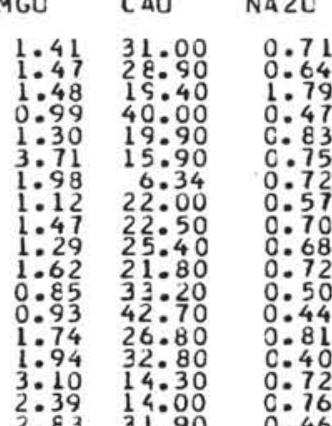
K20

P205 $\mathrm{CL}$

LITHCLCGY

SITE 371: LAT 37 UE 36 NIN N; LCNG 5 JEG 15 NIN E; DEFTH 2826 M (ANAL WALLACE) SAMPLE
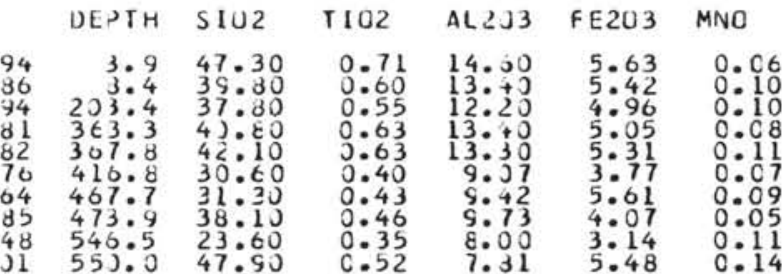

MGO

CAO NA2C
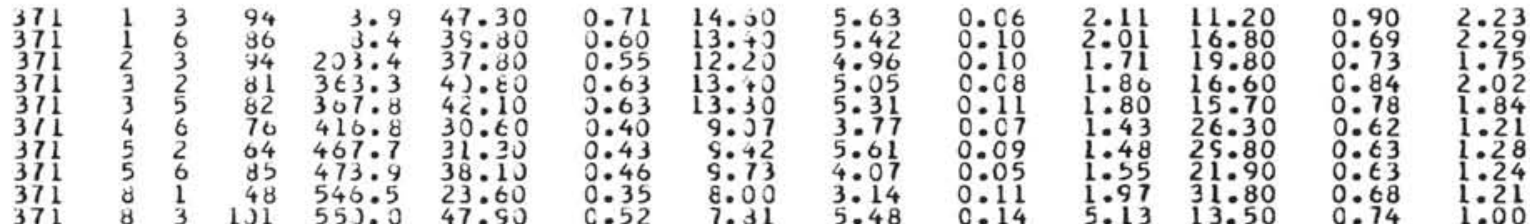

$1: 45$
$1: 51$
$2: 03$
$0: 73$
$1: 10$
$1: 20$
$1: 94$
$0: 62$
$1: 01$
$0: 91$
$1: 00$
0043
$0: 52$
$0: 90$
$0: 90$
$2: 02$
$2: 01$

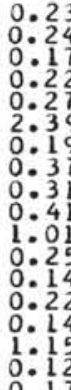

(

YEY NANNO OOLE 0.80 AANNC MARL 0.75 NANNC NARL 1.07 NANNO CLAY 0.67 DOLOMITIC NARL 0.63 NANNO DI ATCM CLAY 0.85 NANNO MARL MARL 0.77 SILTY NANNC MARL 0.92 ARG. NANNC LS: 0.61 NANNO MARL 0.91 ARG. CHALK 0.80 NANNO MARL 0.79 SILIY NANNC CHALK

SITE 372 :LAT 40 UEG 2 NIN N;LCNG 4 DEG 48 MIN E;CEPIH $2734 \mathrm{~N}$ (ANAL hALLACE)

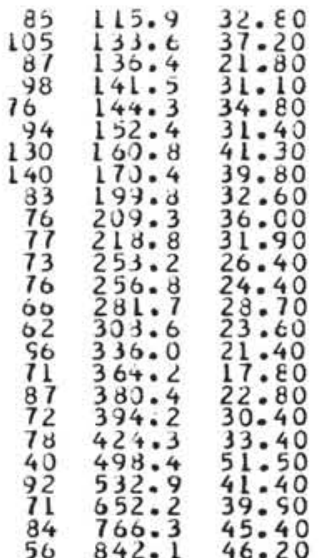

$0.43 \quad 10.20$
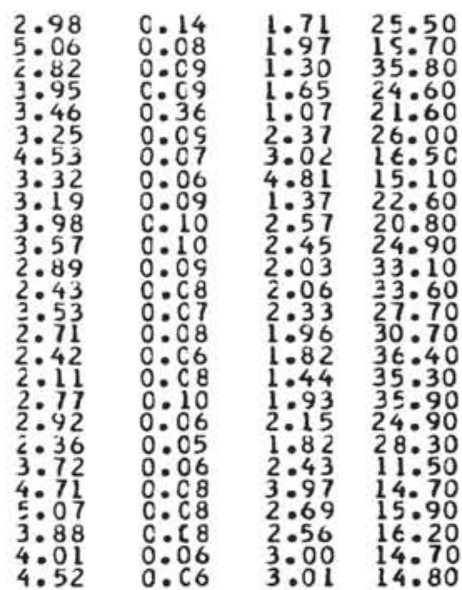

NA2O

K20

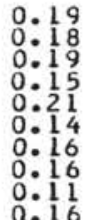

$\mathrm{CL}$

LITHCLCGY

1.21 CALC. MUD \& CLAY

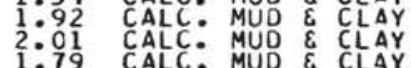
2.56 CALC: MUD \& CLAY 2.58 CALC MUD \& CLAY

SITE 375: LAT 34 CEG 46 NIN N; LCAG 31 CEG 46 NIN E; DEPIH 1914 M (ANAL WALLACE) SAMPLE DEPTH SIO2 TIO

\begin{tabular}{|c|c|c|c|}
\hline 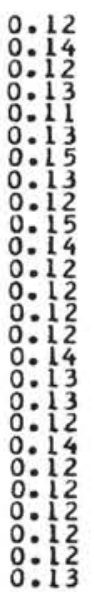 & $\begin{array}{l}0.13 \\
1: 09 \\
1: 39 \\
1: 31 \\
1: 07 \\
1: 05 \\
1: 08 \\
0: 89 \\
1: 20 \\
1: 32 \\
1: 33 \\
1: 12 \\
1: 27 \\
1: 16 \\
1: 34 \\
1: 02 \\
1: 00 \\
1: 49 \\
1: 05 \\
1: 18 \\
0: 58 \\
1: 100 \\
0: 91\end{array}$ & $\begin{array}{l}\text { AANNC } \\
\text { NANNO } \\
\text { NANNO } \\
\text { NANNO } \\
\text { NANNO } \\
\text { NANNO } \\
\text { DCLCM } \\
\text { OOLCM } \\
\text { DOLOM } \\
\text { MARL } \\
\text { MARL } \\
\text { MARL } \\
\text { MARL } \\
\text { AARL } \\
\text { MARL } \\
\text { MARL } \\
\text { MARL } \\
\text { MARL } \\
\text { MARL } \\
\text { MARL } \\
\text { MARL } \\
\text { NANNO } \\
\text { NANNO } \\
\text { NANNO } \\
\text { NANNO } \\
\text { NANN }\end{array}$ & $\begin{array}{l}\text { MARL } \\
\text { MARL } \\
\text { MARL } \\
\text { MAR } \\
\text { MARL } \\
\text { MARL } \\
\text { IIIC } \\
\text { IIIC } \\
\text { IIIC }\end{array}$ \\
\hline
\end{tabular}

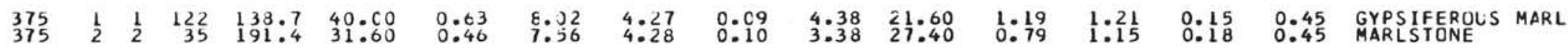



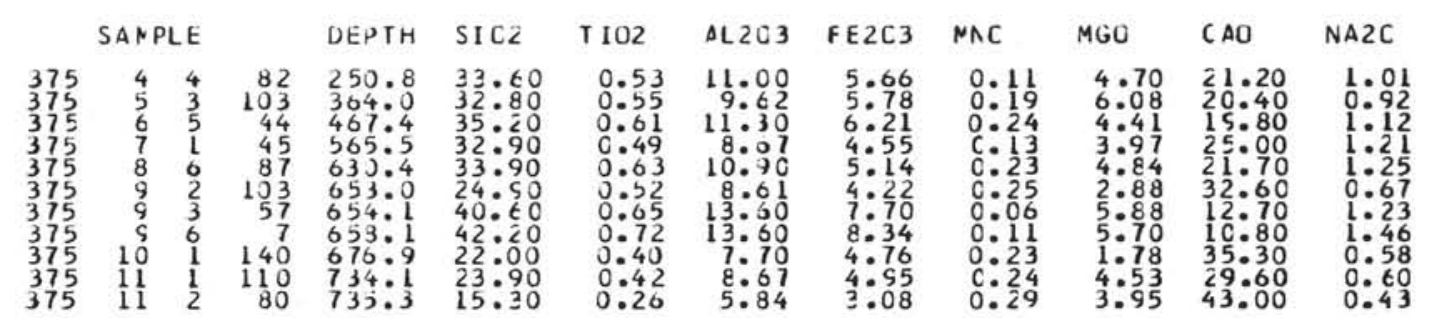

SITE 376: LAT

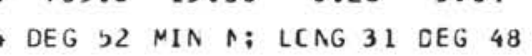

NIN E; CEPTH ZLIOI M (ANAL WALLACE)

SAMPLE

$$
\text { DEPTH SIUL TIU2 ALZU3 FEZU3 MNO }
$$$$
\text { MGO }
$$

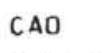

NA2C K2O

$\begin{array}{rr}376 & 1 \\ 376 & 2 \\ 376 & 3 \\ 376 & 4 \\ 376 & 5 \\ 376 & 6 \\ 376 & 7 \\ 376 & 8 \\ 376 & 9 \\ 376 & 10 \\ 376 & 12 \\ 376 & 13 \\ 376 & 15 \\ 376 & 16\end{array}$

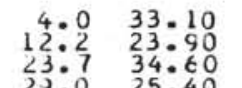

$\begin{array}{rr}0.62 & 10.30 \\ 0.43 & 7: 66 \\ 0.65 & 11: 00 \\ 0.52 & 8: 58 \\ 0.54 & 5: 11 \\ 0.40 & 7: 12 \\ 0.54 & 10: 60 \\ 0.49 & 10: 30 \\ 0.46 & 8: 09 \\ 0.47 & 8.80 \\ 3: 39 & 8: 45 \\ 0.61 & 12.00 \\ 0.38 & 6.15 \\ 0.44 & 7.64\end{array}$

5.83
4.95
5.84
4.22

4.22
$5: 14$
3.62

$\begin{array}{ll}5.62 \\ 5 \\ 5\end{array}: 43$

$3: 70$
3.89

3.89
$4: 25$
$5: 16$
$2: 97$
$3: 70$
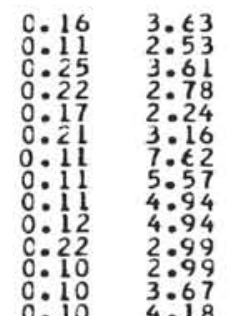

20.10
26.60
18.10
32.30
$28: 20$
33.80
15.60
16.30
19.30
18.90
23040
12.40
30.20
26.20

1.00
0.87
$1: 01$
$0: 70$
$0: 70$
$0: 65$
0.91
$0: 89$
1.17
1.08
0.94
1.07
1.04
0.98

SITE 379:LAT 43 DEG J MIN N:LLNG 36 CEG 1 1IN E; CEPIT $2171 \mathrm{M}$ (ANAL MALLACE)$$
\text { SANPLE }
$$

DEPTH SIU2

$\mathrm{TIO} \quad \mathrm{AL} 2 \mathrm{C} 3$ FE2C3 MNC

MGC

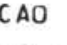

NA2O K2

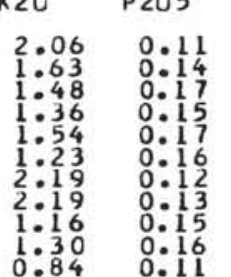

$\mathrm{CL}$

LITHOLCGY

$\begin{array}{llllll}379 A & 1 & 3 & 79 & 3.8 & 40.7 C \\ 379 A & 4 & 5 & 60 & 32: 6 & 44: 30 \\ 379 A & \epsilon & 6 & 86 & 53.4 & 57.80\end{array}$

$\begin{array}{rrrrrr}379 A & \epsilon & 6 & 86 & 53: 4 & 57: 80 \\ 379 A & 7 & 4 & 165 & 59: 2 & 53: 30 \\ 379 A & 8 & 5 & 93 & 7309 & 53: 10 \\ 379 A & 9 & 2 & 41 & 15: 4 & 46: 00\end{array}$

$\begin{array}{llllll}379 A & 10 & 2 & 41 & 15.4 & 46.00 \\ 35 & 155 & 85.2 & 55.60\end{array}$

$0.81 \quad 13.30$

7.22
$7: 32$
$6: 59$
$6: 26$

$\begin{array}{lll}0.72 & 14: 50 & 6: 20 \\ 0.62 & 13: 35 & 6: 26\end{array}$

$0.62 \quad 14.50$

0.7214 .40

$379 A$

$\begin{array}{llllll}379 A & 13 & 6 & 48 & 119: 5 & 45: 20 \\ 379 A & 14 & 6 & 30 S & 128: 8 & 73: 10 \\ 379 A & 15 & 2 & 78 & 132: 8 & 56: 10\end{array}$

$\begin{array}{llll}1 & 1105 & 93.6 & 54.80 \\ 2 & 1405 & 100.4 & 54.80\end{array}$

$0.72 \quad 14.40$

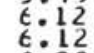

$379 \mathrm{~A}$

$379 A$
$379 A$

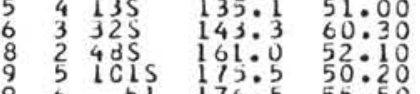

14.7

$0.76 \quad 14: 50$

$0.70 \quad 13.60 \quad 6.21$

$6: 32$
6.59
$6: 21$
$6: 30$
6.14

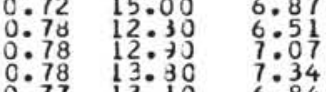

$379 \mathrm{~A}$

$\begin{array}{cccc}2 & 275 & 189: 3 & 51: 00 \\ 2 & 271 & 205: 1 & 44: 10\end{array}$

7.07
$7: 34$
6.84

$\begin{array}{lll}0.75 & 12.90 & 6: 98 \\ 0.94 & 13: 30 & 7: 47 \\ 0.68 & 14.40 & 6: 77\end{array}$

$0: 11$
$0: 18$
$0: 07$
$0: 13$
$0: 16$
$0: 13$
$0: 13$
$0: 12$
$0: 11$
$0: 11$
$0: 15$
$0: 12$
$0: 14$
$0: 21$
$0: 14$
$0: 19$
$0: 13$
$0: 18$
$0: 118$
$0: 20$
$0: 16$
$0: 14$
$0: 19$
$0: 112$
$0: 10$
$0: 16$
$0: 14$
$0: 10$
$0: 07$
$0: 17$
$0: 17$
$0: 20$
$0: 14$
$0: 14$
$0: 11$
0.12

$4: 33 \quad 10.30$

1.56

$1: 33$

P205 CL

0.2

LCMITIC NARLSICAE

0.35 DOLOMITIC MARLSICNE

0.21 DCLCMITIC MARLSTCNE

0.18 MARLSICNE

0.23 MARLSTCNE 0 SAPROPELIC CLAYSTUNE

$0: 26$

0.13 MARLSTONE

LITHCLCGY

1.32

$1: 175$

1.56

1.66
1.31
1.87

$1: 90$

0.11

0.14

0.20

$0: 13$

0.15

$0: 16$

1
0.99
0.98
0.99

NANNO MARL

0.64 DCLCMIIIC MARLSICNE

0.53 DCLCMITIC MARLSTCNE

0.47 SANOY MARLSTONE

0.28 MARLSTCNE

$\begin{array}{llllll}379 A & 24 & 6 & 145 & 212.3 & 45.70\end{array}$

$379 \mathrm{~A}$

3794
$379 A$

$379 A$
$379 A$

$379 A$
$379 A$

$379 A$

$379 A$
$379 A$
$379 A$

P205 CL

CL LITHOLCGY

$0.19 \quad 0.86$ MUD

0.19 0:15 MUD

0.17

0.16

0.16

0.17

0.18

0.20

0.18

$0: 18$

0.22

$0: 14$

0.14

$0: 17$

$0: 14$

0.21

0.18

0.27

0.16
0.17 MUD

0.21 NuD

0

0.17 Mu

0.19 Mud

0.24 MUD

$0_{0}^{0.32}$ MUD

$0: 11$ MUD

0.17 NuD

$0: 16$ MLD 0 OICMACECLS MUD

$0: 17$ NUD

18 MUD

$0: 15$ NUD CALAREOUS MUI

$0: 35$ MuD

0.16 NLD

0.16 MUD

0.26 MUD

0.0 NUD 0 SEEREIDE

0.36 MLD 
SITE 380:LAT 12 DEG O MIN N:LCNG 29 DEG 37 NIN E; DEPTH 2115 M (ANAL WALLACE)

DEPTH SIU2 TIO2 ALLU3 FE2O3 MN

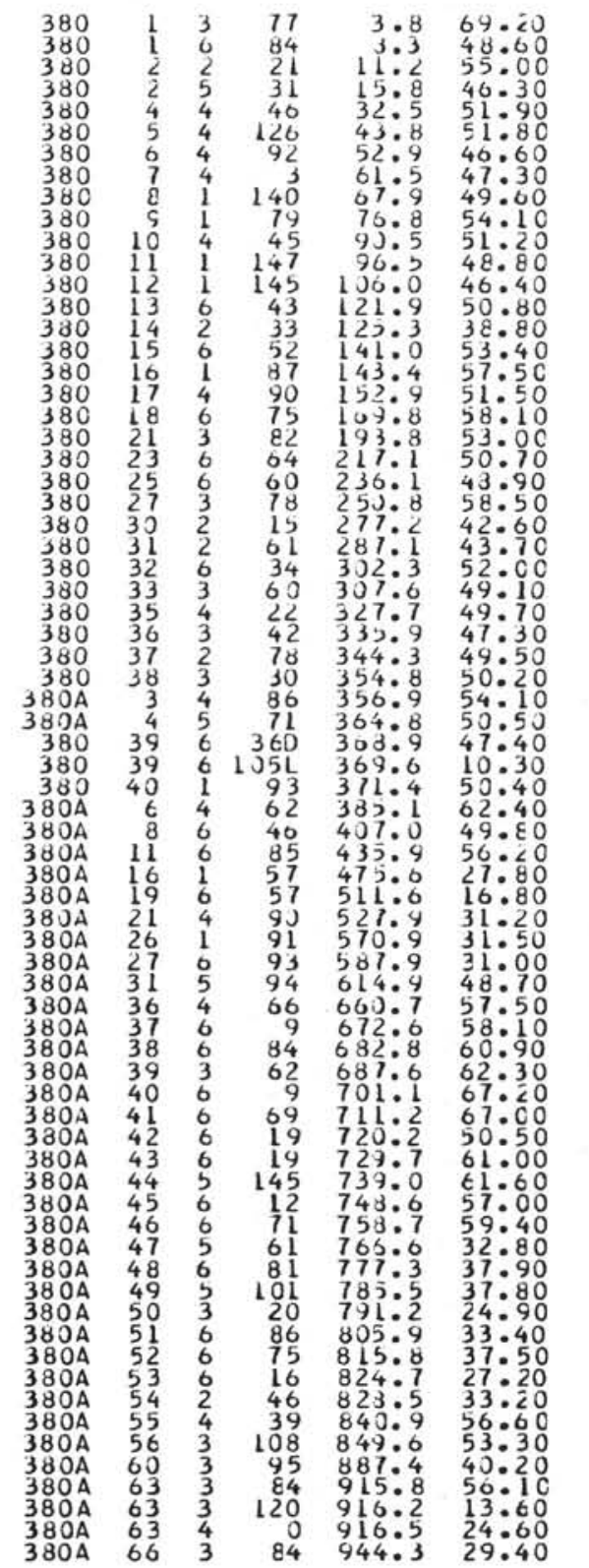

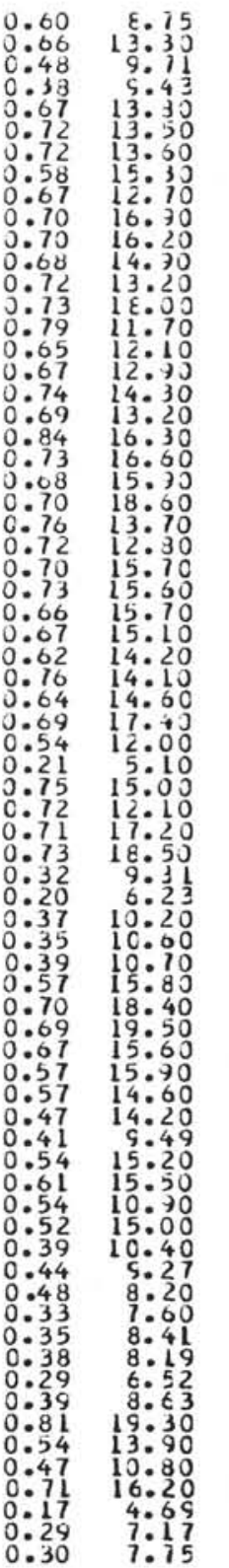

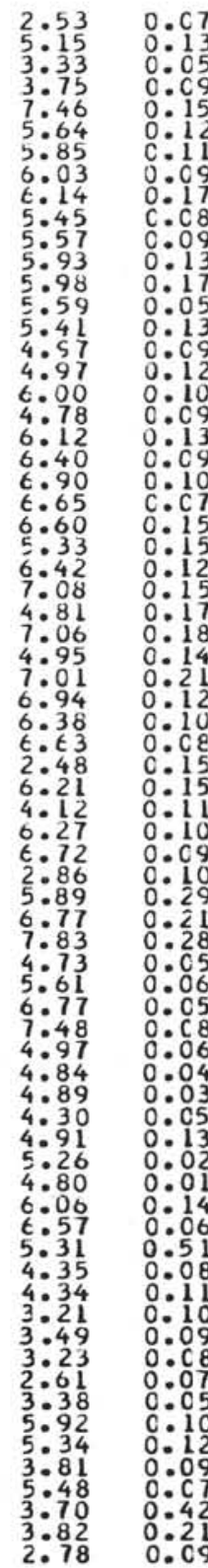

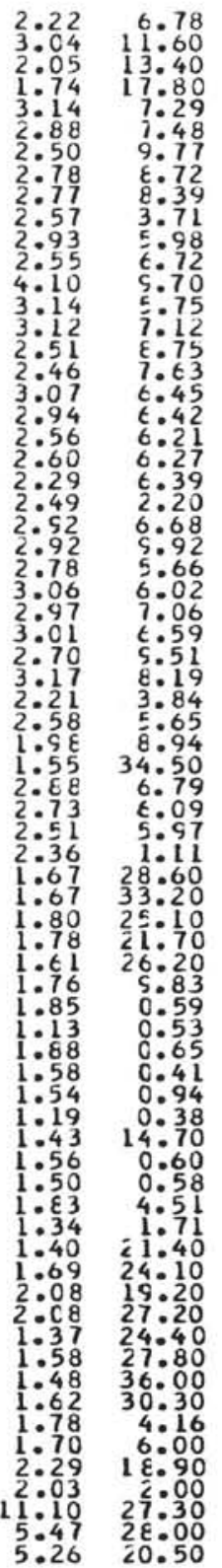

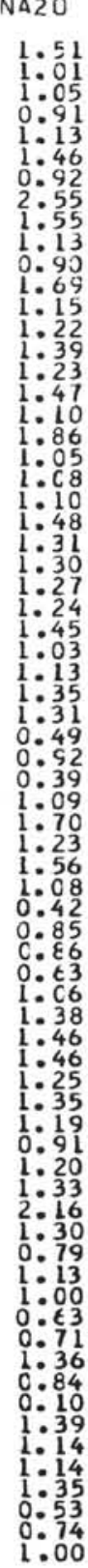

$\mathrm{K} 20$ 1.57
2.16 $\frac{1}{2}: 75$ 2.08

2.36

3.14

2.46

2.

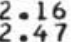

$2_{2}^{2}: 11$

2
2.62
3.62

$\frac{2}{2}: 49$

2.06

$2: 72$

2.44

$2: 23$

$2: 06$

$3: 06$

2.92

${ }_{1}^{2}: 90$

$1: 43$

1
2
2

$2: 46$

$2: 02$

$1: 75$

$2: 16$

$2: 16$

1.66

$1: 25$

$1: 16$
$1: 03$
$1: 3$

$\begin{array}{ll}1 & 032 \\ 2 & 32 \\ 2 & 02 \\ 1 & 02 \\ 2 & 91\end{array}$

$\frac{1}{2}: 912$

0.63
P205 CL

0.15 0.25 MUD

0.12 0.28 MUD

$\begin{array}{lll}0.16 & 0.24 & \text { NLD } \\ 0.15 & 0.42 & \text { DIAICNACECLS MUD } \\ 0.13 & 0.73 & \text { DIAICMACEOLS MUD }\end{array}$

0.1

0.12

$0: 14$

0.14

016

0.18

0.13

$0: 17$

$0: 13$

$0: 19$

$0: 14$

0.12

0.17

$0: 13$

$0: 16$

$0: 19$

$0: 14$

0.100

0.01

0.11

0.09

0.08

0.08

$0: 08$

0.07

$0: 19$

$0: 12$

$0: 14$

$0: 14$

$0: 07$

0.13

0
0:54 DIATOMACECLS MUD

0.77 MUD

: 19 MUA IATMACEOLS MUD

$1: 04$
0.67
0

$0: 93$ MUD

.62 MLD

0.69 MUD

$0: 67$ NUD

GREENISH BLACK CLAY

0.78 MUD

0.95 diatonaceols muo

0.51 dIATCMACEOLS MUD

0.55 MUD

$1: 78$ DIDTCMACECls MUD

$1: 84$
$1: 53$
$0 R$ ORGANIC MUD

0.84 BRChN CLAY

0.85 MUD

$: 07$ MUD

0.97 SEERREIDE

:04 SEEKREIDE

:21 SE SERREIDE

8 CIATCNACEOLS MUD

:13 Diarcmaceous mud

178 DIATCMACECLS MUD

:01 DIAICNACECL MUD

$2: 14$ DIATOMACEOLS MUD

S8 DIATOMACEOLS MUD

:25 DIATCNACEOLS MARL

19. SEERREIDE MARL

136 SEEKREIDE MARL

$1: 02$
1

$1: 33$ CLAY

. 11 diatomaceols stale

$1: 03$ SHALE

102 OCLOMIIIC MARL 


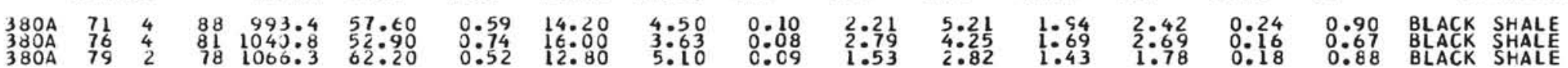
SITE 382 :LAT34 DEG 25 MIA NiLCNG 56 DEG 32 MIN W;CEPTH 5\$37 M (ANAL hALLACE)

SAMPLE DEPTH SIU2 TIO2 AL203 FE2O3 MNO MGO CAO NA2

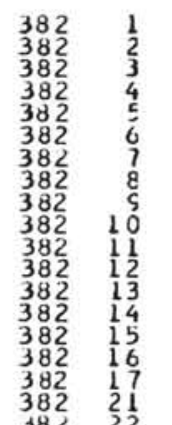

$382 \quad 223$
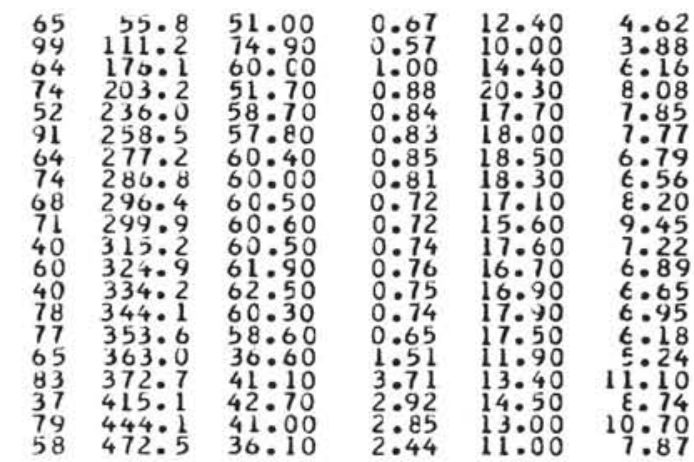

$0: 10$
$0: 06$
$0: 11$
$0: 10$
$0: 21$
$0: 04$
$0: 12$
$0: 05$
$0: 05$
$0: 04$
$0: 04$
$0: 06$
$0: 07$
$0: 08$
$0: 80$
$0: 15$
$0: 25$
$0: 14$
$0: 14$
$0: 27$
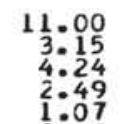

NA2C K2O

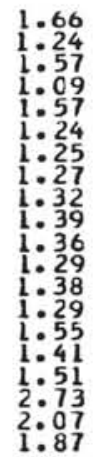

SITE 386: LAT

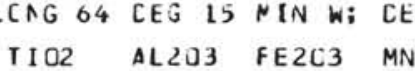

MEPTH 4793 N(WALLACE, BUDD)
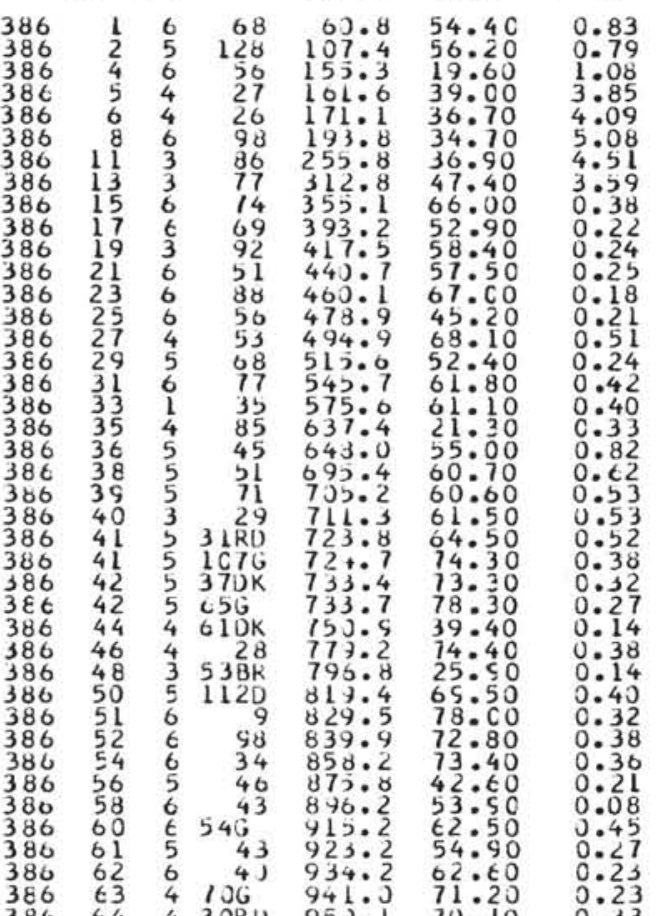

$\begin{array}{rr}18.90 & 8.03 \\ 17: 70 & 7: 08 \\ 6: 53 & 4: 42 \\ 12: 50 & 13: 70 \\ 12: 40 & 13: 20 \\ 10: 30 & 12: 50 \\ 11: 00 & 11: 80 \\ 11: 80 & 13: 50 \\ 9: 90 & 4: 77 \\ 6: 49 & 1: 70 \\ 6: 14 & 1: 95 \\ 5.47 & 2.05\end{array}$

$2: 05$

$5: 10$
12.10

$6: 56$
5: 69

9.96
$6: 07$
19.90

$5: 06$
1.92

$\frac{1}{3} .427$

3.45
$1: 53$
7.99

$\begin{array}{ll}15.8 \mathrm{C} & 8.26 \\ 14.70 & 8: 38\end{array}$

$14: 40$
$12: 70$

घ: 77

$5: 40$
$5: 14$

4.38

6.98
2.49

4.00

4.88

4.89
5.36

3.33
5.29

$\begin{array}{rr}11: 70 & 5.78 \\ 5.6 \mathrm{C} & 6: 32\end{array}$

$\begin{array}{ll}5.63 & 5.53 \\ 4.42 & 5.88\end{array}$
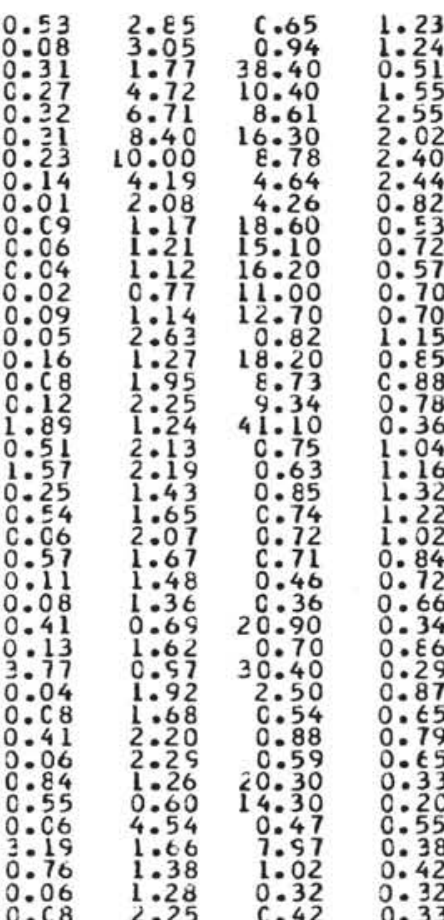

P205 CL

$$
\text { LITHOLOGY }
$$

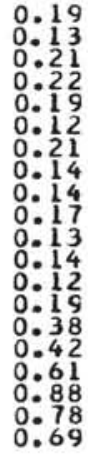

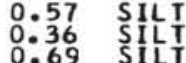

0.87 CLAY

0.55 CLAY

0.60 CLAY

0.61 CLAY

0.77 CLAY

0.52 CLAY

0.53 CLAY

0.95 CLAYY clar

1.16 ZEOLITIC SIIIY CLAY
0.46 ZEOLITIC SILTY CLAY

$\mathrm{CL}$

LITHCLCGY
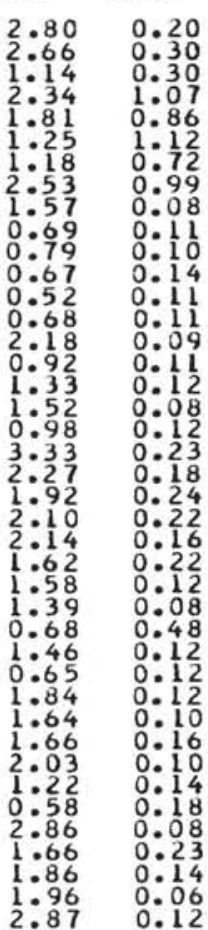

1.07 CLAY

$1: 48$
0.82

: 30 EASALIIC SAAD/CLAY

67 EASALTIC SAND/CLAY

.22 eASALTIC SANCICLAY

1.29 MARLY SIL: OOZE

0.55 CALC. CLAYSTLNE

0.45 CALC CLAYSTCNE

0.50 CLAYSTONE

0.48 CLAYSITNE

0.64 SILIY CLAYS IENE

.26 CLAYSTCNE

$0: 31$ ZEOLITIC CLAYSICNE

0.30 ZECLIIIC CLAYSTCN

0.22 GREEN CLAYSICNE

O 26 GREEN CLAY STCN

0.55 CARK MARLY CLAYSTINE

0.32 CLAYSTCNE

. 32 CORK MARLY CLAY.

0.34 CLAYSTCAE

0.32 CLAYSICNE

0.33 MARLY CLAYSTONE

0.38 GREEN CLAYSTCNE

0.37 CARK CIAYSTCNE

0.45 CLAYSICNESA SAND

0.35 RADIOLARI
0.36 CLAYSTCNE 
SITE 387: LAT 32 DEG 19 NIN N; LCNG 67 LEE 40 NIN W; CEPTH E128 M (ANAL. BUDD) DEPTH SIUZ TIUZ AL 203 FEZU3 MNO
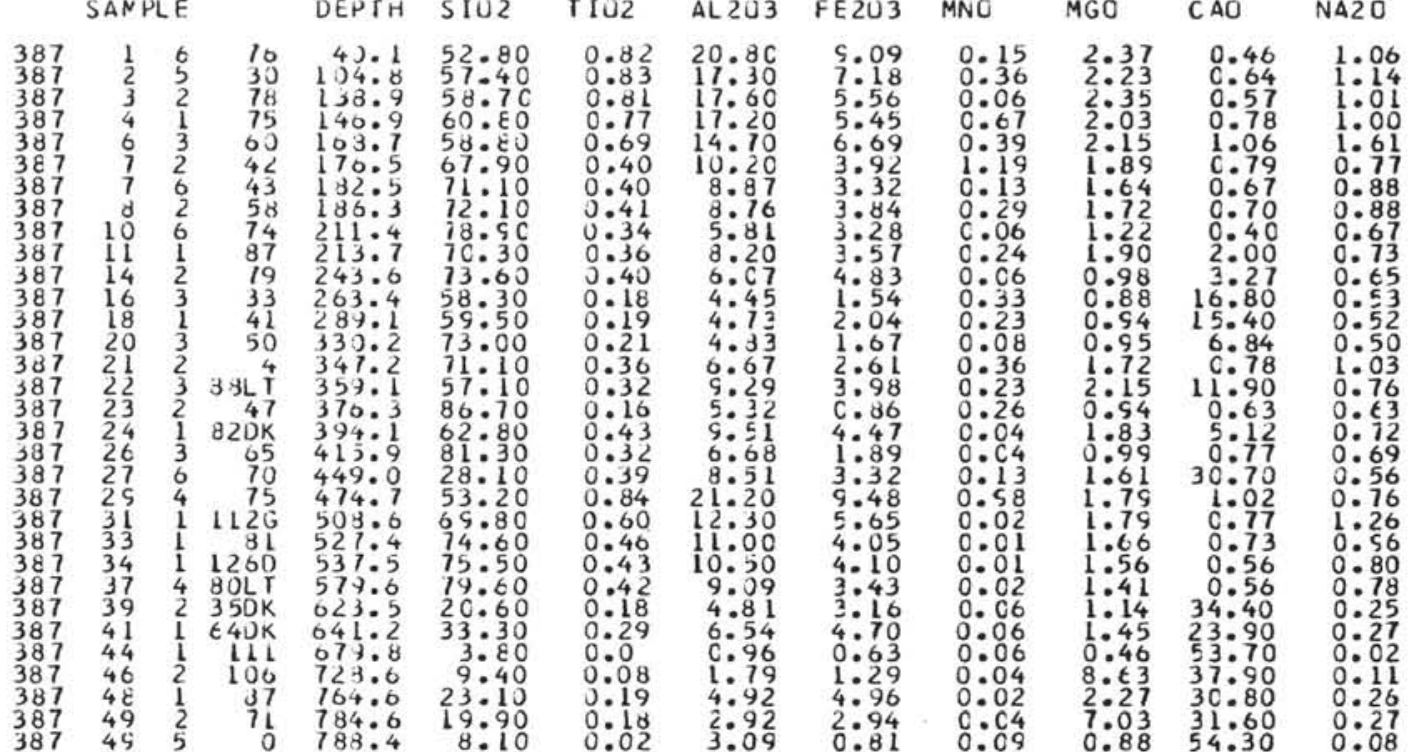

SITE 391: LAT 28 OEG 14 NIN $N$;LCNG 75 LEG 37 MIN h:DEFTH 4 SE 4 N (ANAL BUDDI

SAMPLE

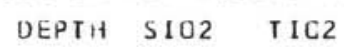

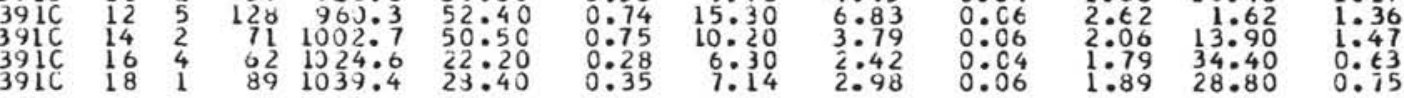

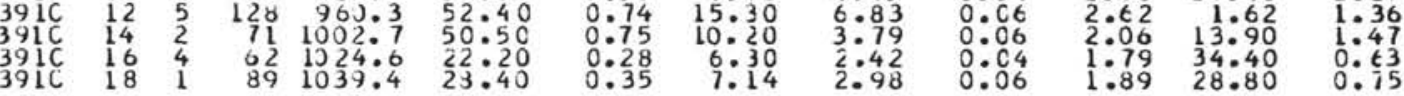

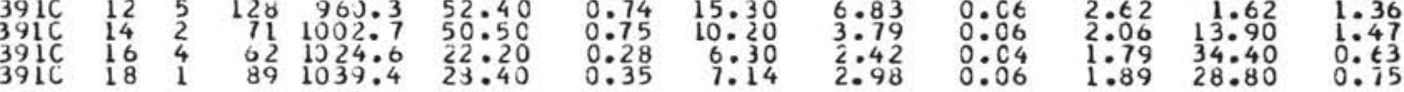

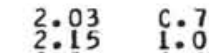

C. 75

NA2O

P205

SITE 417: LAT 25 DEG $7 N I N ~ N$; LCNG 68 DEC 3 MIN $W$; DEFTH $54 E \varepsilon-5482$ (WALLACE)

DEPTH SIC 2
MGO

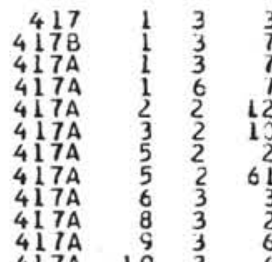

TIC2 AL2C3 FE2C3 MNC

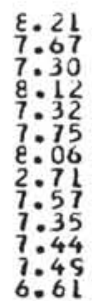

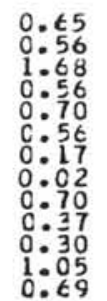

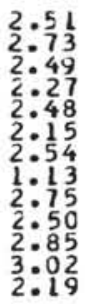

CAO NA2O

0.84
0.69
0.69
0.50
0.58
0.50
0.55
0.96
0.69
0.54
0.64
0.65
0.65

K20
2.87
$2: 10$
$2: 00$
$2: 05$
$2: 85$
$1: 43$
$1: 35$
$1: 28$
$1: 06$
$1: 18$
101
0.54
0.64
0.89
$1: 54$
$1: 32$
0.67
$1: 31$
$1: 14$
$1: 30$
$3: 37$
$1: 88$
$1: 51$
$1: 57$
$1: 60$
$1: 22$
1078
$0: 19$
$0: 42$
$1: 47$
1.25
0.34

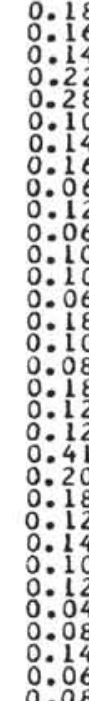

$0: 16$
$0: 14$
$0: 22$
$0: 28$
$0: 10$
$0: 14$
$0: 16$
$0: 06$
$0: 12$
$0: 06$
$0: 10$
$0: 10$
$0: 118$
$0: 18$
$0: 10$
$0: 08$
018
$0: 12$
$0: 12$
$0: 41$
$0: 20$
$0: 18$
$0: 12$
$0: 14$
$0: 10$
$0: 12$
$0: 04$
$0: 08$
$0: 14$
$0: 06$
0.08

1.32
1.53
$1.80 O L I T I C$ CLAY 1.87
1.27
1.04 1.06 RADIOLARIAN NUD 1.64
1.66
RADIOLARIAN MOL MUDOY RAIAN MUC 1.78 MUDDY RAD. UOLE 0.29 SILICECUS CLAYSTONE 0.56 SILICEUS CLAYSTCN 0.72
0.57 RAOICLARIAN MLOSTONE 0.59 CLAYSICNE 0.63 CLAYSTCNE 0.34 CALCAREOLS CLAYSTONE 0.44 GREEA CLAYSTCNE .42 BLACK CLAYSTCNE 0.31 GRAY CLAYSICNE 0.37 LIMESTONE 0.21 LIMESTCNE 0.12 LIMESICNE

\section{.24 LIMESTCNE}

P205 CL

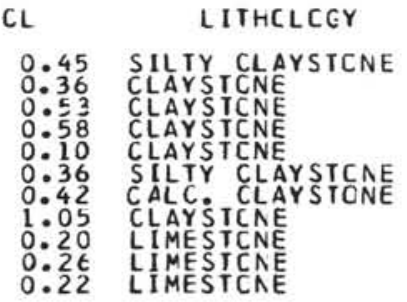

0.22 LIMESTCKE 1.300 .08

23
19
07
11
69
07
03
03
99
99
01
00
022

P205

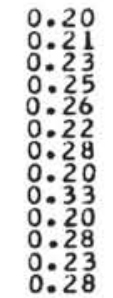

$\mathrm{CL}$

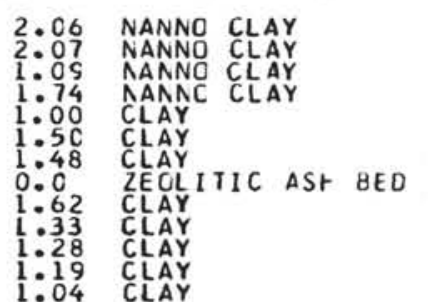




\begin{tabular}{|c|c|c|c|c|c|c|c|c|c|c|c|c|c|c|c|}
\hline \multicolumn{3}{|c|}{ SAMPLE } & & & 102 & & & INO & IGC & & 20 & 21 & & L & \\
\hline $\begin{array}{l}7 \mathrm{7A} \\
7 \mathrm{7A} \\
7 \mathrm{7A} \\
7 \mathrm{AA} \\
7 \mathrm{AA} \\
7 \mathrm{7A} \\
7 \mathrm{7D} \\
7 \mathrm{~A}\end{array}$ & $\begin{array}{rr}12 & 3 \\
13 & 1 \\
14 & 2 \\
15 & 3 \\
16 & 3 \\
17 & 1 \\
18 & 2 \\
19 & 3 \\
20 & 1 \\
22 & 1 \\
7 & 2 \\
23 & 1 \\
8 & 1 \\
8 & 1 \\
15 & 1 \\
10 & 1 \\
10 & 2 \\
11 & 1 \\
12 & 2 \\
12 & 2 \\
12 & 2 \\
14 & 3 \\
14 & 3 \\
15 & 1 \\
16 & 1 \\
17 & 1 \\
17 & 2 \\
17 & 3 \\
18 & 1 \\
19 & 1 \\
20 & 1 \\
20 & 1 \\
20 & 2 \\
21 & 2 \\
21 & 3 \\
21 & 3 \\
21 & 4\end{array}$ & 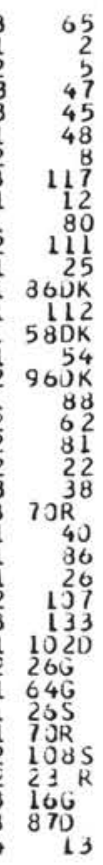 & 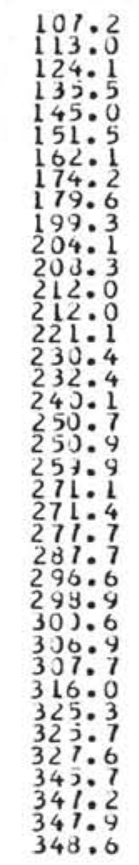 & 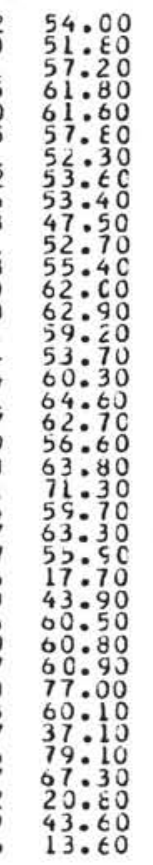 & $\begin{array}{l}8: 76 \\
0: 80 \\
0: 68 \\
0: 62 \\
0: 59 \\
0: 65 \\
0: 91 \\
0: 86 \\
0: 83 \\
0: 82 \\
0: 81 \\
0: 76 \\
0: 53 \\
0: 72 \\
0: 75 \\
0: 78 \\
0: 67 \\
0: 55 \\
0: 65 \\
0: 73 \\
0: 82 \\
0: 40 \\
0: 61 \\
0: 59 \\
0: 11 \\
0: 13 \\
0: 48 \\
0: 49 \\
0: 54 \\
0: 28 \\
0: 09 \\
0: 20 \\
0: 08 \\
0: 36 \\
0: 19 \\
0: 34 \\
0.13\end{array}$ & 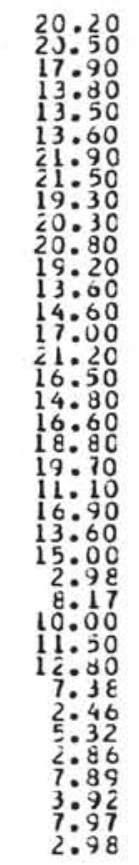 & 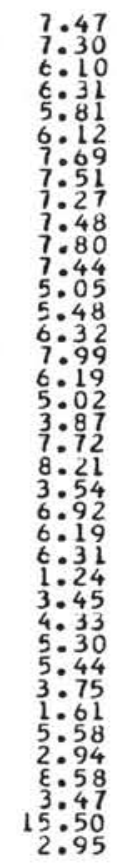 & $\begin{array}{l}1: 20 \\
1: 68 \\
0: 70 \\
0: 44 \\
0: 45 \\
0: 13 \\
0: 83 \\
0: 12 \\
0: 94 \\
0: 37 \\
0: 58 \\
1: 22 \\
1: 13 \\
1: 88 \\
1: 25 \\
0: 09 \\
1: 13 \\
0: 06 \\
0: 03 \\
0: 06 \\
0: 15 \\
0: 04 \\
0: 10 \\
1: 43 \\
0: 31 \\
0: 16 \\
0: c 6 \\
0: 04 \\
0: 06 \\
0: 03 \\
0: 11 \\
0: 21 \\
0: 10 \\
0: c 4 \\
0: 32 \\
0: c 7 \\
0: 39\end{array}$ & $\begin{array}{l}2: 49 \\
2: 18 \\
1: 85 \\
1: 81 \\
3: 73 \\
1: 70 \\
2: 03 \\
2: 90 \\
1: 90 \\
2: 17 \\
1: 62 \\
1: 33 \\
1: 54 \\
1: 58 \\
1: 55 \\
1: 58 \\
1: 14 \\
1: 23 \\
1: 48 \\
1: 66 \\
1: 03 \\
1: 50 \\
3: 74 \\
0: 88 \\
2: 93 \\
3: 57 \\
4: 61 \\
4: 59 \\
1: 64 \\
0.60 \\
1: 61 \\
0: 94 \\
2: 55 \\
1: 30 \\
2: 49 \\
0: 96\end{array}$ & 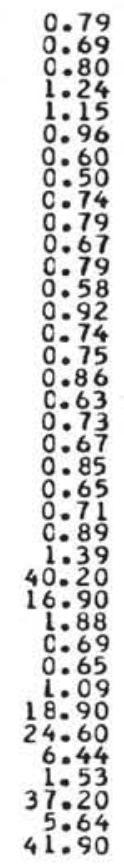 & 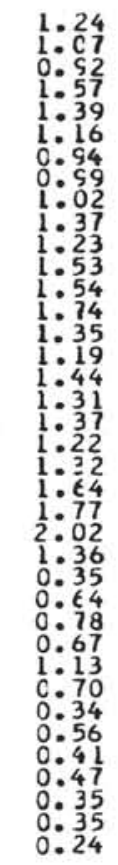 & 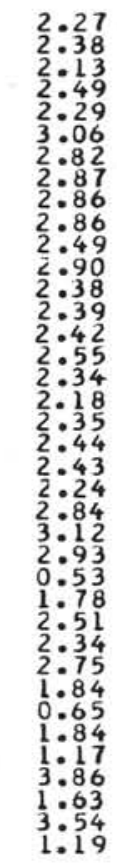 & 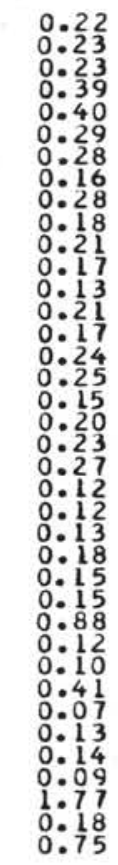 & $\begin{array}{l}1: 24 \\
1: 09 \\
1: 69 \\
1: 19 \\
1: 66 \\
1: 41 \\
0: 84 \\
0: 94 \\
0: 90 \\
0: 78 \\
0: 86 \\
0: 97 \\
1: 02 \\
0: 83 \\
0: 67 \\
1: 18 \\
0: 80 \\
0: 72 \\
0: 64 \\
1: 05 \\
0: 68 \\
0: 72 \\
0.64 \\
1: 12 \\
1: 10 \\
1: 04 \\
1: 45 \\
0: 35 \\
0: 59 \\
0: 42 \\
0: 49 \\
0: 72 \\
0: 58 \\
1: 18\end{array}$ & 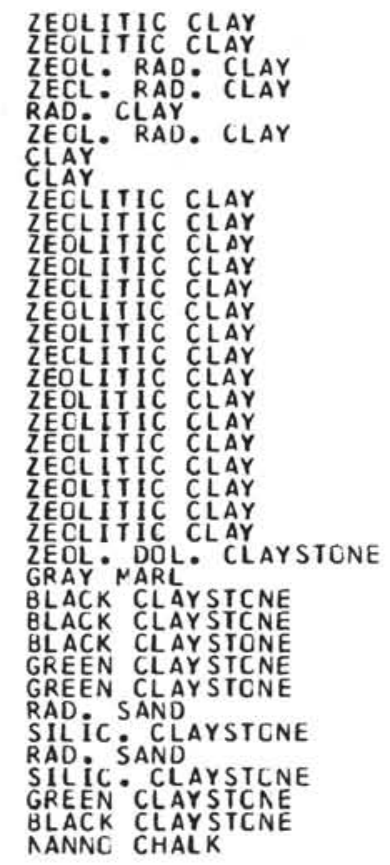 \\
\hline & & N1I & LC & 0 & II & & M & 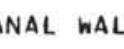 & & & & & \\
\hline & & & & & 02 & & & MN & MGO & $C A$ & 120 & & & & \\
\hline & $\begin{array}{ll}8 & 1 \\
10 & 1 \\
12 & 1 \\
12 & 2 \\
13 & 1 \\
13 & 2 \\
13 & 2 \\
13 & 2\end{array}$ & $\begin{array}{r}141 \\
92 \\
131 \\
26 \\
60 \\
8 \\
115\end{array}$ & & $\begin{array}{l}52: 00 \\
51: 10 \\
18: 20 \\
21: 00 \\
44: 00 \\
64: 90 \\
64: 50\end{array}$ & $\begin{array}{l}0.84 \\
0: 44 \\
0: 11 \\
0: 14 \\
0: 34 \\
0: 45 \\
0.51\end{array}$ & $\begin{array}{r}20.10 \\
5: 16 \\
3: 67 \\
4: 26 \\
7: 47 \\
9: 00 \\
10.10\end{array}$ & $\begin{array}{r}8.14 \\
4: 88 \\
2: 50 \\
3: 10 \\
6: 94 \\
10: 50 \\
6.22\end{array}$ & $\begin{array}{l}0.99 \\
0: 04 \\
0: 31 \\
0: 30 \\
0: 33 \\
0: 136 \\
0.16\end{array}$ & $\begin{array}{l}2.58 \\
2: 48 \\
1: 09 \\
1: 30 \\
2: 28 \\
2: 96 \\
2.60\end{array}$ & $\begin{array}{r}0.52 \\
0: 59 \\
37: 90 \\
35: 10 \\
16.30 \\
0.47 \\
3.07\end{array}$ & $\begin{array}{l}0.7 \\
0: 3 \\
0: 4 \\
0: 5 \\
0: 7 \\
c .6\end{array}$ & & & $\begin{array}{l}0: 8 \\
1: 0 \\
0: 7 \\
0: 6 \\
0: 6 \\
0: 8 \\
0.6\end{array}$ & $\begin{array}{l}\text { CLAY } \\
\text { CLAAY } \\
\text { CLAY EY } \\
\text { CLAYYEY } \\
\text { NANAO C } \\
\text { CLAY } \\
\text { CLAY }\end{array}$ \\
\hline & $420: L A$ & & $\mathrm{MIN}$ & $\wedge \wedge$; & & & & & & & & & & & \\
\hline & & & & & & & & & MG & & & & & & \\
\hline & $\begin{array}{rr}1 & 2 \\
1 & 2 \\
2 & 3 \\
2 & 3 \\
3 & 2 \\
4 & 3 \\
5 & 2 \\
5 & 2 \\
6 & 2 \\
7 & 2 \\
7 & 2 \\
7 & 2 \\
8 & 3 \\
5 & 3 \\
10 & 3 \\
11 & 2 \\
12 & 1 \\
12 & 1 \\
13 & 5 \\
13 & 5\end{array}$ & 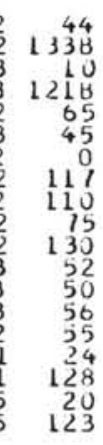 & 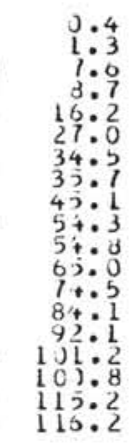 & 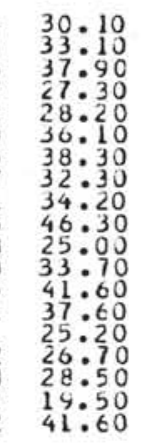 & $\begin{array}{l}0: 31 \\
0: 40 \\
0: 39 \\
0: 38 \\
0: 28 \\
0: 38 \\
0: 34 \\
0: 27 \\
0: 26 \\
0: 39 \\
0: 17 \\
0: 20 \\
0: 28 \\
0: 26 \\
0: 16 \\
0: 14 \\
0: 22 \\
0: 16\end{array}$ & $\begin{array}{l}\epsilon: 4 c \\
1: 60 \\
7: 40 \\
6: 80 \\
5: 20 \\
7: 00 \\
6: 70 \\
4: 40 \\
1: 20 \\
3: 81 \\
3: 00 \\
5: 60 \\
5: 20 \\
4: 20 \\
3: 62 \\
3: 50 \\
3: 18\end{array}$ & $\begin{array}{r}8: 41 \\
11: 50 \\
11: 80 \\
9: 96 \\
7: 84 \\
7: 88 \\
9: 31 \\
7: 24 \\
7: 53 \\
10: 80 \\
8: 05 \\
6: 77 \\
9: 85 \\
11: 10 \\
3: 58 \\
6: 37 \\
6: 05 \\
14: 10\end{array}$ & $\begin{array}{l}0: 4 \\
6: 4 \\
1: 5 \\
5: 2 \\
0: 4 \\
0: 4 \\
0: 5 \\
0: 3 \\
1: 1 \\
0: 6 \\
1: 8 \\
1: 7 \\
0: 5 \\
0: 3 \\
0\end{array}$ & $\begin{array}{l}1: 98 \\
2: 23 \\
2: 54 \\
1: 73 \\
1: 78 \\
2: 43 \\
2: 84 \\
2: 16 \\
2: 24 \\
3: 80 \\
2: 03 \\
1: 57 \\
3: 03 \\
1: 29 \\
1: 45 \\
1: 76\end{array}$ & 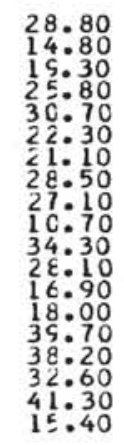 & $\begin{array}{l}0: \\
0: \\
0: \\
0: \\
0: \\
0: \\
0: \\
0: \\
0: \\
0: \\
0:\end{array}$ & & $\begin{array}{l}0 \\
0 \\
0 \\
0 \\
0 \\
0 \\
0 \\
0 \\
0 \\
0 \\
0 \\
0 \\
0 \\
0 \\
0 \\
0 \\
0\end{array}$ & $\begin{array}{l}0: 2 \\
0: 1 \\
0: 2 \\
0: 2 \\
0: 1 \\
0: 1 \\
0: 3 \\
0: 1 \\
0: 2 \\
0: 3 \\
0: 3 \\
0: 4 \\
0: 3 \\
0: 1 \\
0: 1 \\
0: 3\end{array}$ & 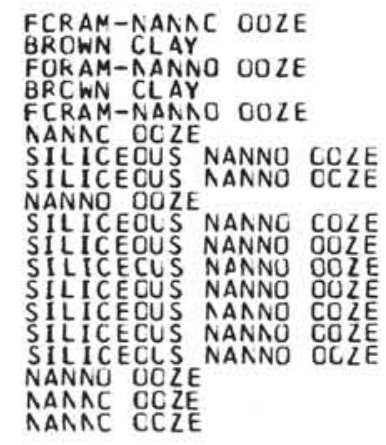 \\
\hline
\end{tabular}


SITE 424: LAT O JEG 30 MIN $\wedge$; LCNG 86 JEG 8 MIN W; DEPTH 2688 N (ANAL TEKRANA) SANPLE ULPTH SICZ TIUZ ALZC3 FEZC3 MNO MGO CAU NA2C
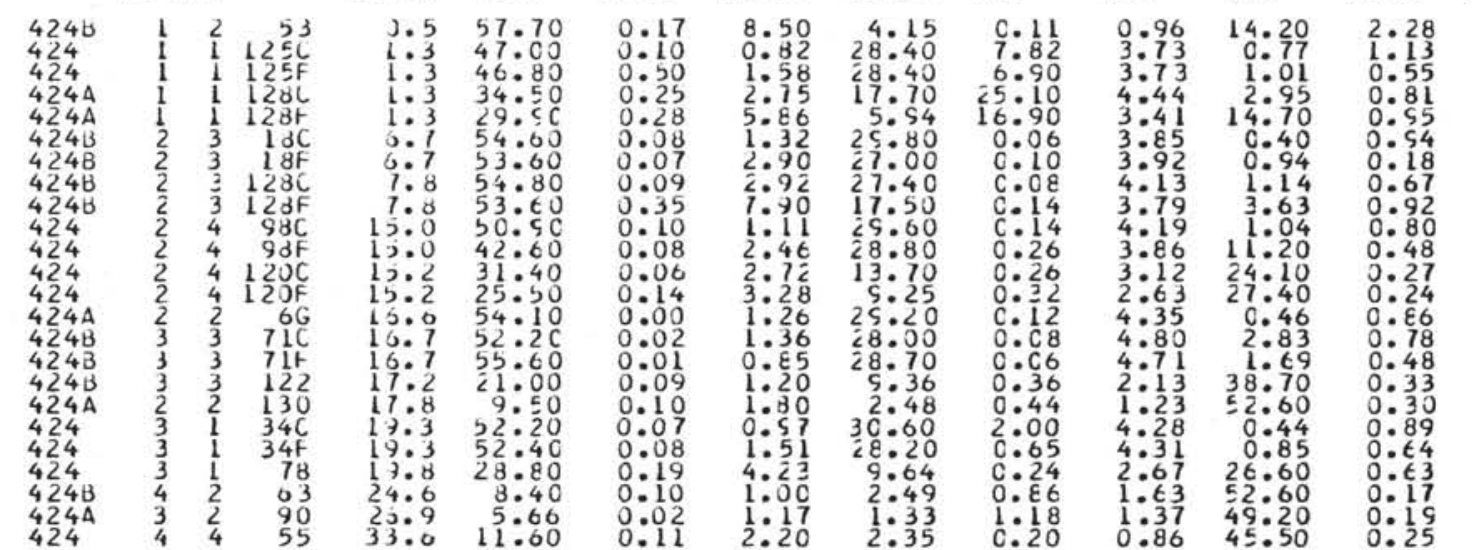

K20

P205 $\mathrm{CL}$

LITHCLOGY

SITE 425: LAI L DEG 24 NIN N; LCNG 86 JEE 4 MIN W; DEPIT $2 E 56$ M (ANAL TERFANA)
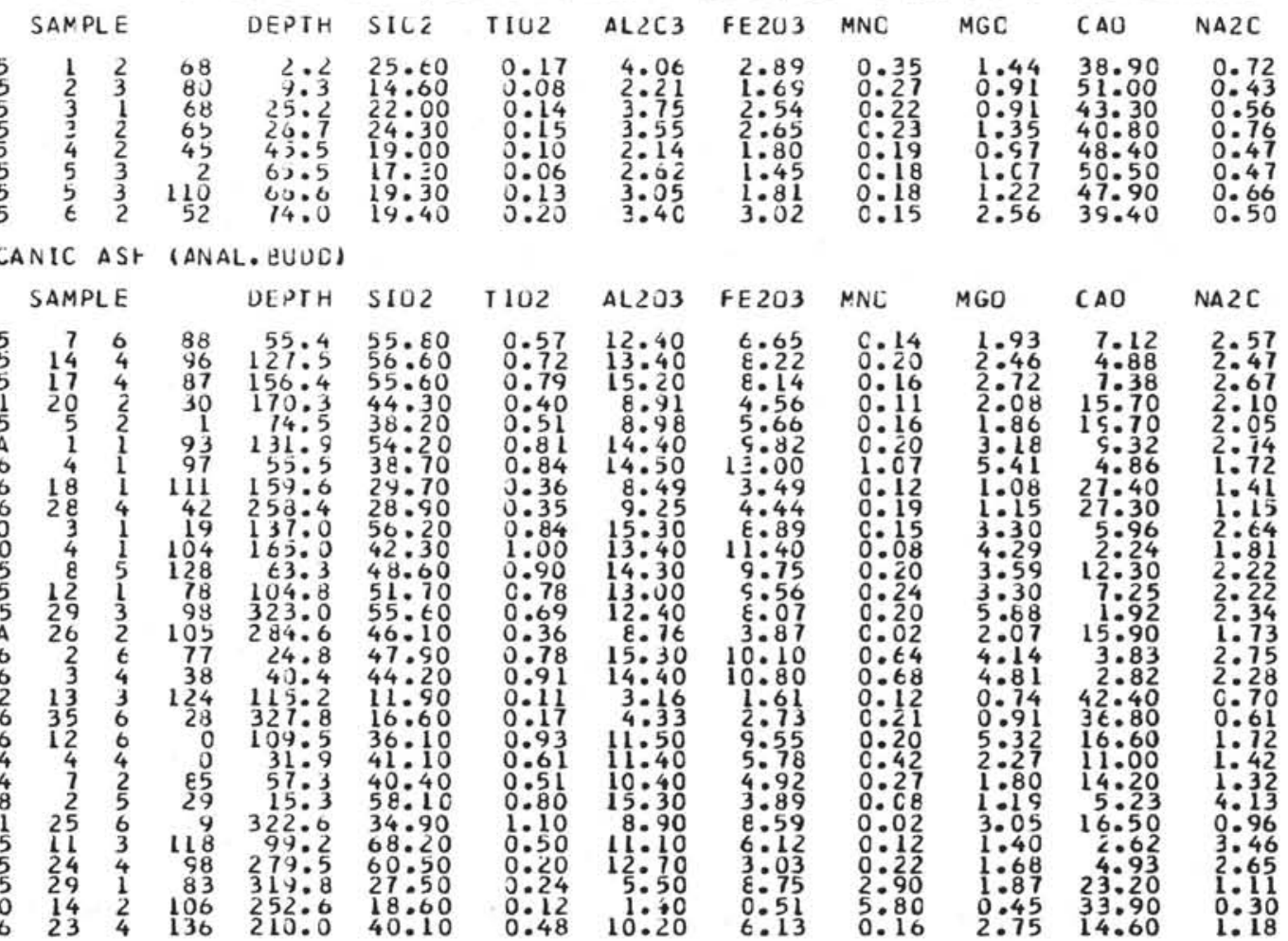

\section{(a)}

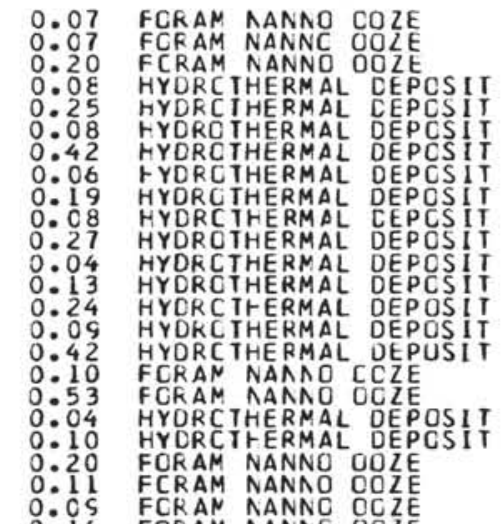

OOS FCRAN NANNC OCZE
0.16 FORAM NANNO OCZE

P205 CL

CL LITHCLOGY

O:54 SHLICEOUS AANO DOZE

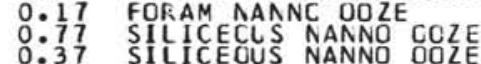

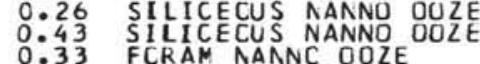

0.20

\section{LithCLCGY}

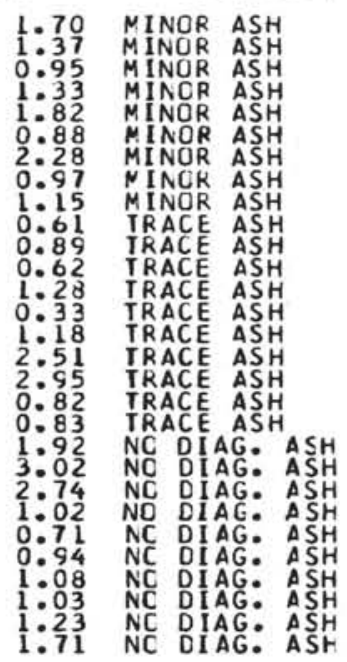


TABLE 3 - Continued

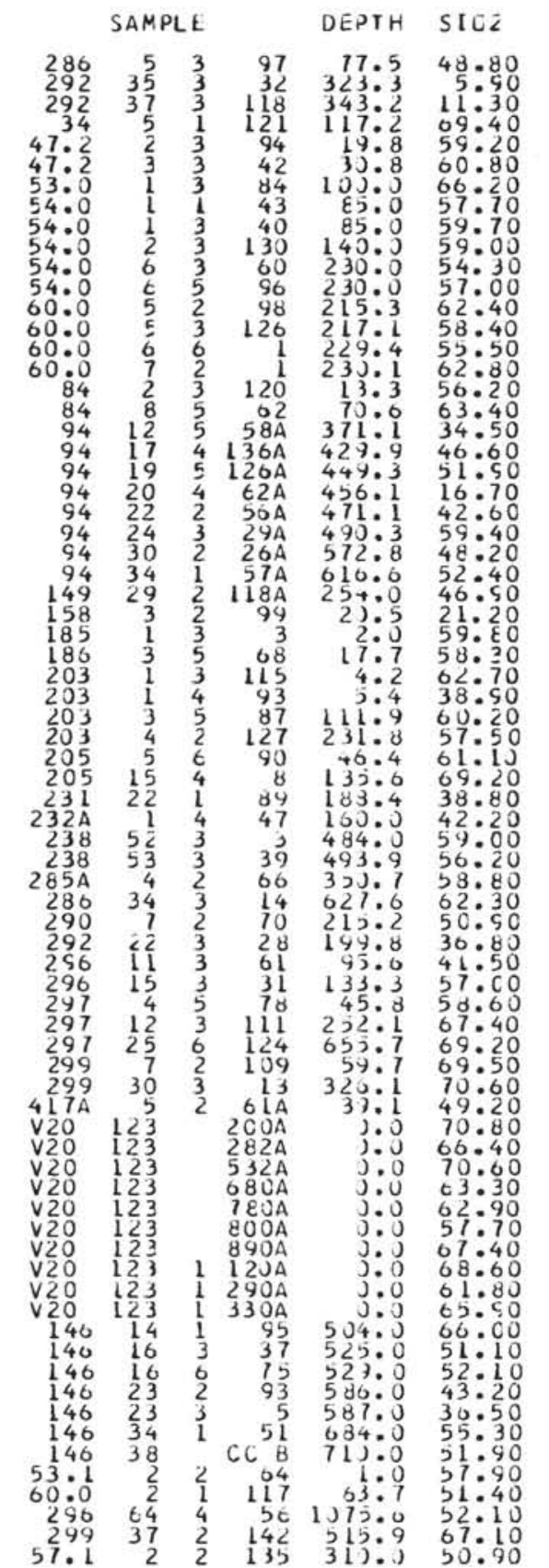

\begin{tabular}{|c|c|c|}
\hline T 102 & $A L \angle C 3$ & $F E 203$ \\
\hline $\begin{array}{l}0: 90 \\
0: 09 \\
0: 07 \\
0: 21 \\
0: 14 \\
0: 32 \\
0: 40 \\
0: 56 \\
0: 56 \\
0: 44 \\
0: 34 \\
0: 48 \\
0: 43 \\
0: 52 \\
0: 58 \\
0: 42 \\
0: 76 \\
0: 18 \\
0: 06 \\
0: 00 \\
0: 04 \\
0: 02 \\
0: 08 \\
0: 12 \\
0: 12 \\
0: 22 \\
0: 14 \\
0: 37 \\
0: 75 \\
0: 55 \\
0: 31 \\
0: 27 \\
0: 54 \\
0: 56 \\
0: 70 \\
0: 40 \\
0: 38 \\
0: 40 \\
0: 40 \\
0: 35 \\
0: 56 \\
0: 48 \\
0: 52 \\
0: 17 \\
0: 31 \\
0: 19 \\
0: 06 \\
0: 43 \\
0: 29 \\
0: 33 \\
0: 24 \\
0: 40 \\
0: 35 \\
0: 43 \\
0: 38 \\
0: 54 \\
0: 44 \\
0: 77 \\
0: 62 \\
0: 27 \\
0: 75 \\
0: 37 \\
0: 58 \\
0: 54 \\
0: 74 \\
0: 46 \\
0: 61 \\
0: 90 \\
0: 57 \\
0: 48 \\
0: 44 \\
0: 89 \\
0: 79 \\
1: 85 \\
\end{array}$ & 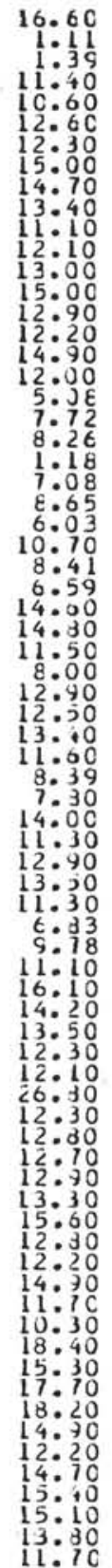 & 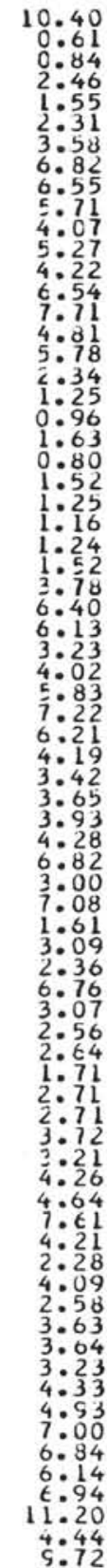 \\
\hline
\end{tabular}

\begin{tabular}{|c|c|}
\hline 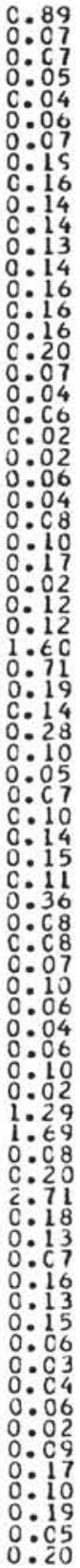 & 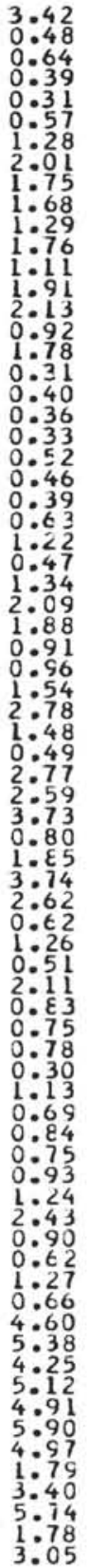 \\
\hline
\end{tabular}

\begin{tabular}{|c|c|}
\hline $\mathrm{NA} 2 \mathrm{C}$ & $k 20$ \\
\hline 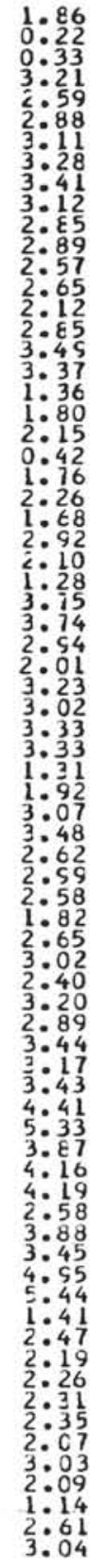 & 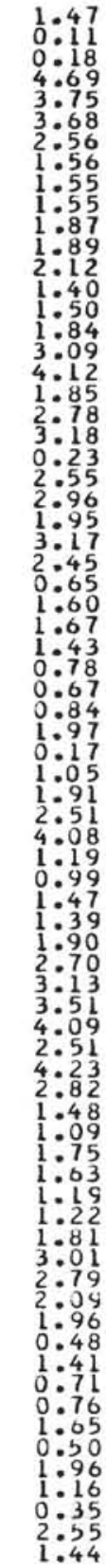 \\
\hline
\end{tabular}

P205

0.45 $0: 11$ 0.06 $0: 12$
$0: 20$
$0: 12$ 0.20 $0: 16$ 0:19 ${ }_{0}^{0}: 12$ $0: 14$ 0.29 ${ }_{0}^{0}: 08$ 0.04 $0: 08$ ${ }_{0}^{0}: 04$ $0: 06$

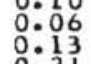
${ }_{0}^{0}: 13$ $0: 10$ 0.16
0
0 $0: 24$ $0: 20$ $0: 14$ $0: 28$

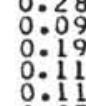
$0: 07$ $0: 11$ 0 $0: 09$ :09 $0: 09$ $0: 14$ $0: 18$ 0.26 $0: 14$ 0.14 $0: 14$ 0.15 $0: 18$ 0.13 0.24
$\mathrm{CL}$

LITHCLCGY

$2: 28$ NC DIAG. ASH

.

$0: 89$ VITRIC ASH

$1: 35$ VIIRIC ASH

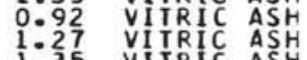

$1: 35$ VIIRIC ASS

1:40 VIIRLC ASH

0 :79 VITRLC ASH

:

$0: 63$ VITRIC ASH

0.70 VITRIC ASH

0.06
0
0

1:08 VIIRLC ASH $0: 74$ VIIRLC ASH

$2: 12$ VIIRIC ASH
$1: 50$ VITRIC ASH

0.84
$2: 18$ VIIRIC ASH
211 VIIC ASH

: 11 VIIRIC ASH

30 VITIC ASH

19 VIRRIC ASA

0.0 VIIRTC ASH

$0: 54$ VIIRR C ASH

0

0.91 VIIRTC ASH

0 VIIRIC AS

0.37 VIITRL ASH

0.64 VIIRIC ASH

0.0 VITRIC ASH

0.45 VIIRIC ASH

0.37 VIRRC ASH

$0: 69$ VITRLC ASH

0.25 VIIRIC ASF

0.30 VIIRIC ASH

0.48 UNCLASSIFIED IYPE

0.50 UNCLASSIFIED IYP

***** UNCLASSIFIEC IYPE

0.36 UNCLASSIEIEC IYPE

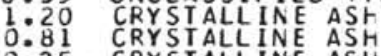

0.015 CRYSTALLINE ASH

0.28 CRYSTALLINE ASH
0.97 CRYSTAL-VITRIC ASH 

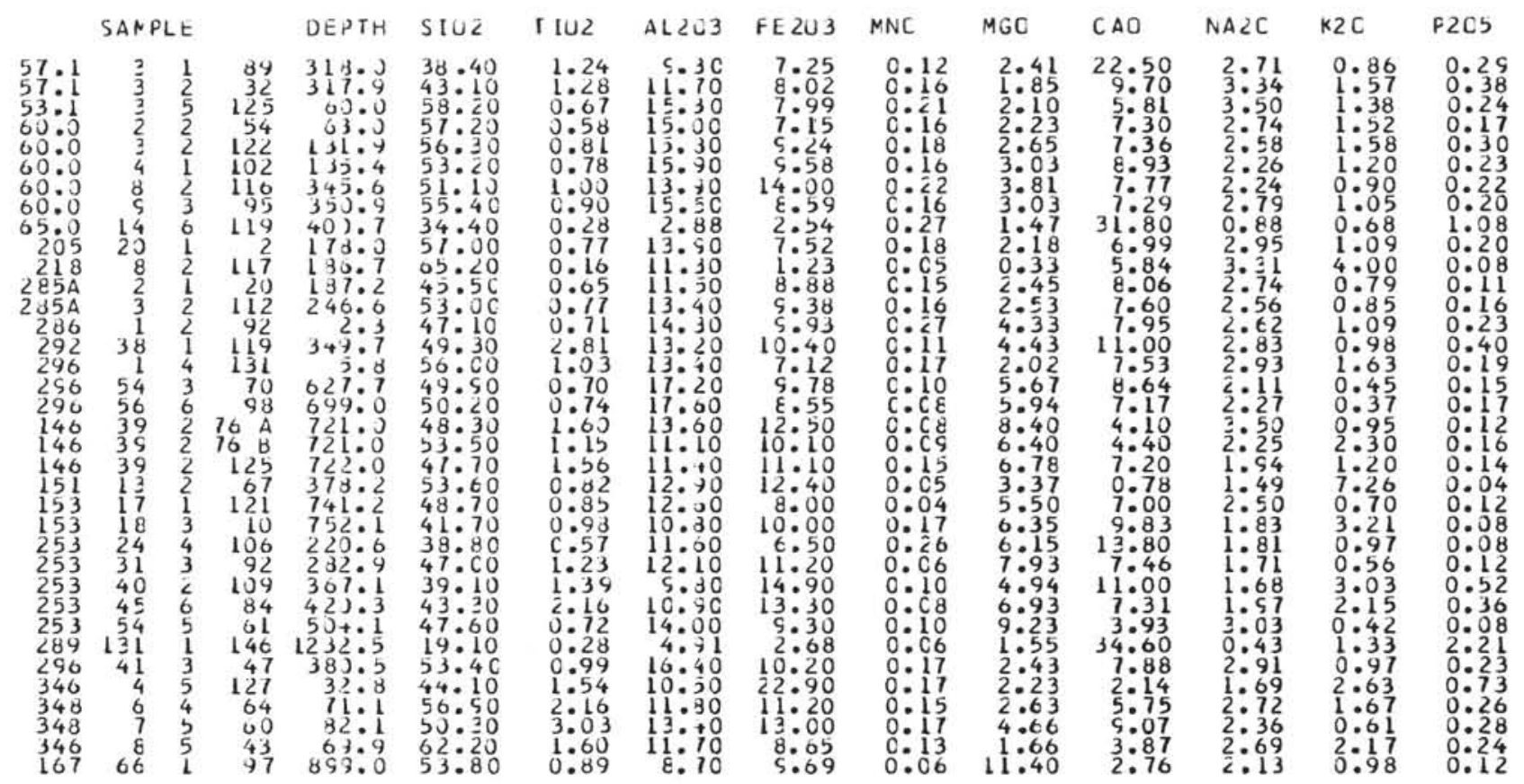

CL

$1: 10$
$0: 86$
$0: 87$
$0: 84$
$0: 32$
$0: 47$
$0: 69$
$0: 46$
$1: 40$
$1: 39$
$0: 68$
$0: 84$
$0: 61$
$2: 26$
$0: 50$
$1: 14$
$0: 40$
$0: 32$
4474
$4 * 47$
$0: 15$
$0: 0$
$0: 0$
$0: 0$
$0: 19$
$0: 58$
$0: 58$
$0: 49$
$0: 65$
$0: 33$
$0: 49$
0056
$0: 53$
$0: 88$
0.42
0.39

0.42
LITHCLCEY

CEFSTAL-VITRIC ASH
CRYSTAL-VITRIC ASH CRYSAL-VITRIC ASH CRYSIAL-VIIRIC ASH CRYSTAL-VIIRIC ASH CRYSIAL-VIIRIC ASH CRYSIAL-VITRIC ASH CRYSIAL-VIIRIC ASH CRYSIAL-VITRIC ASH
CRYSTAL-VIIRIC ASH CRYSIAL-VITRIC ASF CRYSTAL-VITRIC ASH CRYSTAL-VIIRIC ASH
CRYSTAL-VITRIC ASH CRYSIAL-VITRIC ASH EASALIIC AS eASALIIC ASH BASAL IC AS eASALIT ASH easALII IC ASt 政 easal ic as oASAL IIC AS aSALIIC ASH GASALIIC ASH

Note: Given are Site, Core, and Section numbers, and the top of the interval in centimeters. Letters following the interval number are as follows: D or DK $=$ Dark sediment. Lor $\mathrm{LT}=$ Light sediment.

$\mathrm{YL}=$ Yellow

$\begin{array}{ll}\mathrm{G} \text { or } \mathrm{GN} & =\text { Green. } \\ \mathrm{R} \text { or } \mathrm{RD} & =\text { Red. }\end{array}$

$\mathrm{B}$ or $\mathrm{BR}=$ Brown

$\mathrm{A}=$ Volcanic ash.

$\mathrm{S}=$ Sandy or silty layer; this letter is used also for samples analyzed by G. Faure and C. Nardone for strontium isotopes (Site 379 ). $F$ or FRAG = Isolated fragment.

C.F

$\mathrm{C}, \mathrm{F}=$ Coarse and fine sieved separates $(320 \mathrm{mesh})$ from Sites 420,424 , and 425 .

$\mathrm{T}, \mathrm{B}=$ Top and bottom of fragment from Site 323 samples.

$\# 1, \# 2$, etc. $=$ Individual fragments from Site
$1,2,3=$ Individual fragments from Site 322 .

A,B $=$ Different samples from same depth, Sites 146 and 147

***** $=$ For an oxide value, means not determined. 